DEVELOPMENT OF METHODOLOGY FOR DETECTION OF ABNORMAL FUNCTIONING REGION IN HUMAN EXTERNAL ORGANS USING THERMOGRAM ANALYSIS

by

Md Shazzat Hossain

B.Sc. Eng, Dhaka University of Engineering and Technology, Bangladesh (2003)

M.Sc. Eng, Dhaka University of Engineering and Technology, Bangladesh (2006)

\author{
A dissertation \\ presented to Ryerson University \\ in partial fulfilment of the \\ requirements for the degree of \\ Doctor of Philosophy \\ in the program of
}

Electrical and Computer Engineering

Toronto, Ontario, Canada 2015

(C) Shazzat Hossain 2015 


\section{AUTHOR'S DECLARATION FOR ELECTRONIC SUBMISSION OF A DISSERTATION}

I hereby declare that I am the sole author of this dissertation. This is a true copy of the dissertation, including any required final versions, as accepted by my examiners.

I authorize Ryerson University to lend this dissertation to other institutions or individuals for the purpose of scholarly research.

I further authorize Ryerson University to reproduce this dissertation by photocopying or by other means, in total or in part, at the request of other institutions or individuals for the purpose of scholarly research.

I understand that my dissertation may be may be made electronically available to the public. 


\title{
Abstract \\ DEVELOPMENT OF METHODOLOGY FOR DETECTION OF ABNORMAL FUNCTIONING REGION IN HUMAN EXTERNAL ORGANS USING THERMOGRAM ANALYSIS
}

\author{
(C) Shazzat Hossain 2015 \\ Doctor of Philosophy \\ Department of Electrical and Computer Engineering \\ Ryerson University
}

This study has established thermography as a potential diagnostic tool for detecting and parameterizing tumors even at the earlier stage from abnormal local surface thermal features captured by high sensitive infrared cameras without known risk of morbidity. Discrepant thermal features originate not only for tumor's distinguishing physio-bio-thermal features but also for the state of health, resulting in thermography as a useful tool for retrieving information about the tissue's health, thus an efficient screening tool. Accurate linking between hyper functional tissues and thermal pattern could turn the screening tool into a promising parameterizing tool. Human external organs, for example chest, forearm and breast have been modeled, mimicking their shapes, inhomogeneity and deformations to obtain steady-state thermal feature in the tissue interior at healthy state and the computation is extended for tumors buried into healthy tissues for determining abnormal local surface thermal image. Tumor diagnosis parameters have been forecasted from thermogram using an indirect process involving the optimization process. The study has applied gradient (gradient projection method), non-gradient (pattern search method) and 
learning based (genetic algorithm) optimization approaches. Feasibility of the proposed technique is investigated for tumors in human organs.

The local abnormal thermal feature screens earlier stage tumors out and reveal how tumors affect the thermal behaviour and what particular parameters have significant influence on the thermal image. Influential parameters are applied as optimization variables and their influences are also figured out to determine the gradient matrix for the gradient optimization technique. The study has employed bio-heat equations, heat-source model and Artificial Neural Network as governing equation to develop simulated datasets. The simulated dataset is compared with test thermogram to minimize a cost function. In lieu of clinical thermograms, the study has developed pretend thermogram with enveloping the simulated datasets with $\pm 10 \%$ random noise.

This research has tailored optimization algorithms for estimating tumor depth, size, blood perfusion rate, thermal conductivity, and metabolism and the obtained results show good accuracy. The estimated parameters are given to a trained network to reconstruct the thermal feature, thus, validates the performance of the proposed methodology.

Keywords: Infrared thermography, bio-heat transfer, tumor, analytical solution, numerical approach, optimization, evolutionary process 


\section{Acknowledgements}

I would like to express my deepest gratitude to my supervisor, Dr. Farah Mohammadi, for her excellent guidance, caring, patience, and providing me with an excellent atmosphere for doing research. I would never been able to finish my dissertation without the guidance of my supervisor, committee members, help from friends, and support from my family and wife.

I would like to thank my committee members, Dr. Soosan Beheshti, and Dr. Xavier Fernando, from Department of Electrical and Computer Engineering, Ryerson University, for their encouragement, insightful comments, constructive suggestions, but also for the hard questions which incented me to widen my research from various perspectives.

In addition, I am thankful to Dr. Habiba Bougherara, from Department of Mechanical and Industrial Engineering, Ryeron University and Dr. Saeed Mohammadi, from Department of Electrical and Computer Engineering, Purdue University for time spent in the evaluation of my research work and also for their suggestions. I would like to express the deepest appreciation to my committee chair, Dr. Martin Antony, from Department of Psychology, Ryerson University who has the attitude and the substance of a genius.

I thank my fellow graduates, especially Akram Hadeed, for the stimulating discussions and for helping me throughout the work.

I would also like to thank the Department of Electrical and Computer Engineering and the School of Graduate Studies at Ryerson University for their financial support during the course of this work.

I am also grateful to my family and fellow graduate students for their continuous assistance and inspiration throughout the work. 


\section{Table of Contents}

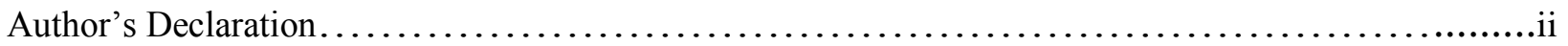

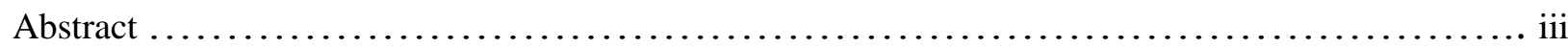

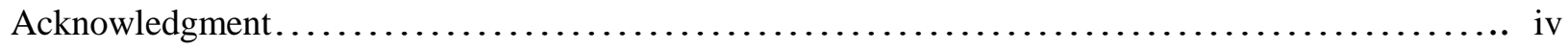

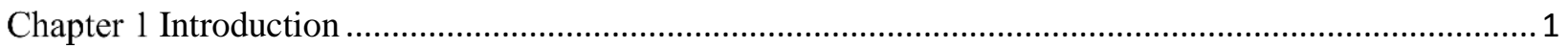

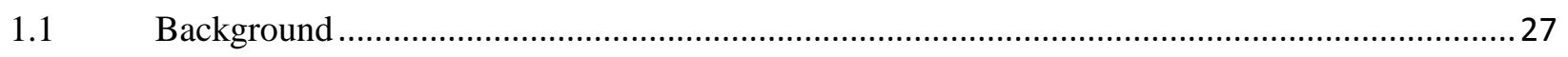

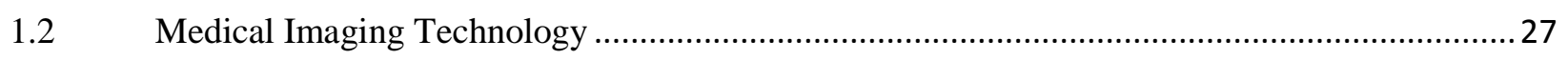

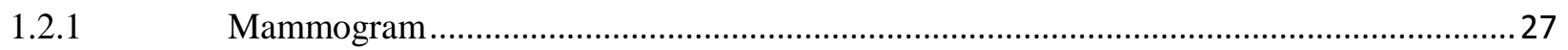

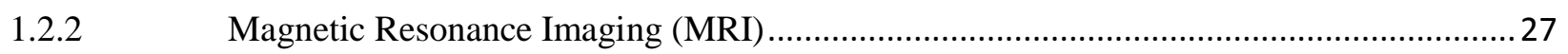

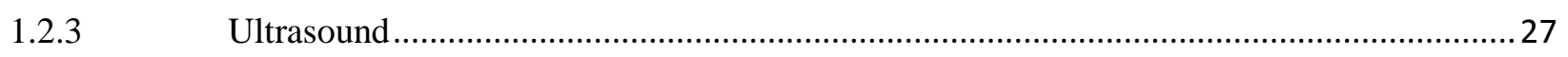

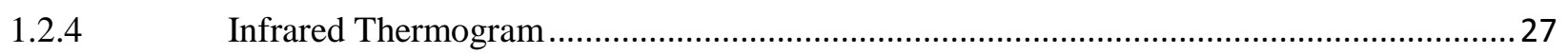

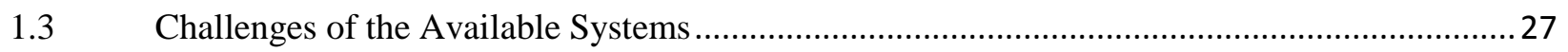

1.4 Computational Tools and Estimation Methodologies in Health Care......................................2 27

1.4.1 Anatomic -accurate Physical Model......................................................................... 27

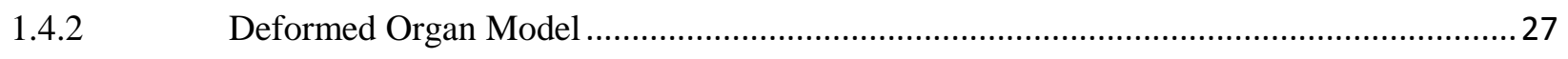

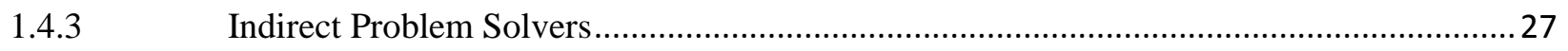

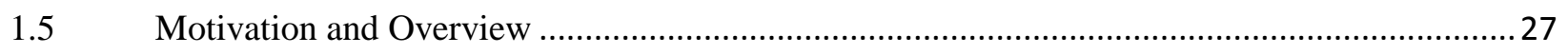

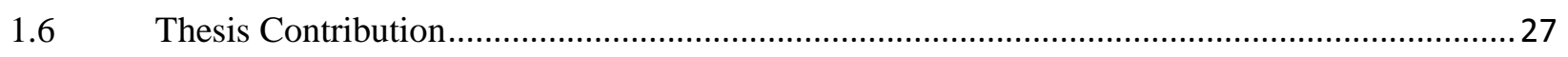

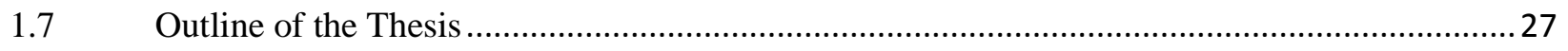

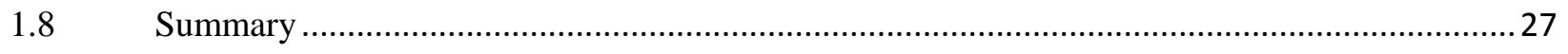

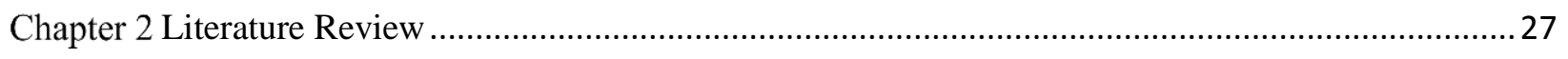

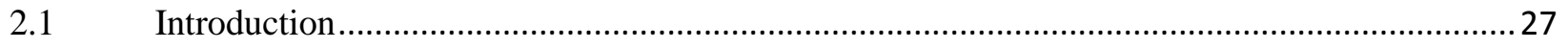

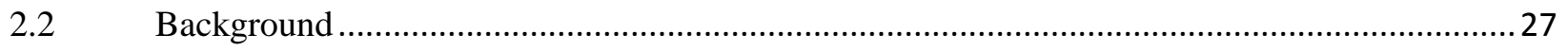

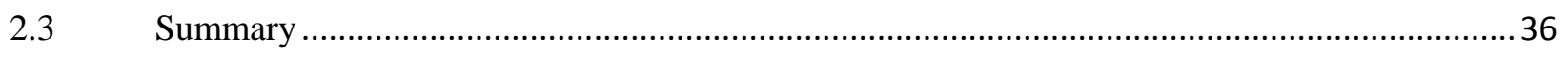

Chapter 3 Thermal Analysis in Healthy Homogeneous Tissues........................................................... 38

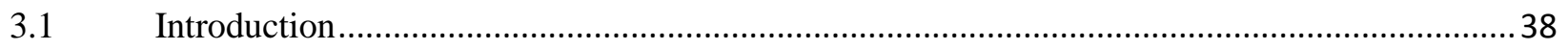

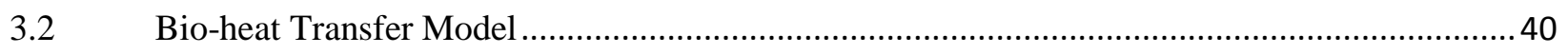

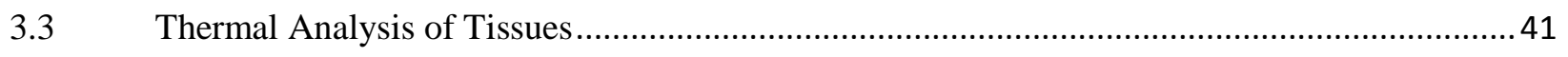

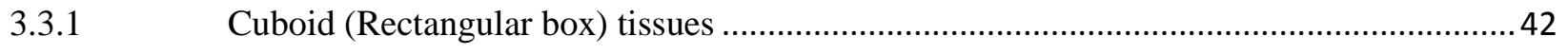

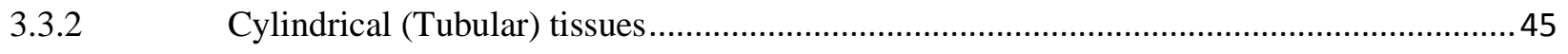

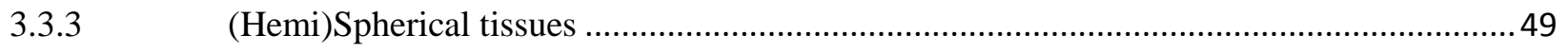




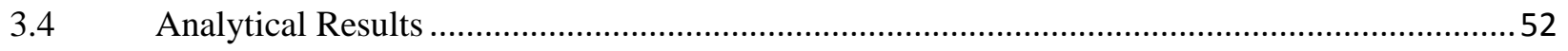

3.5 Comparisons between analytical solutions obtained in different coordinate system ..................54

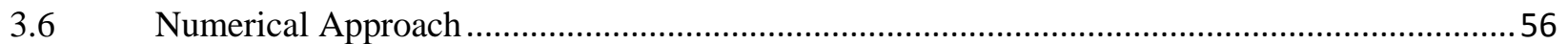

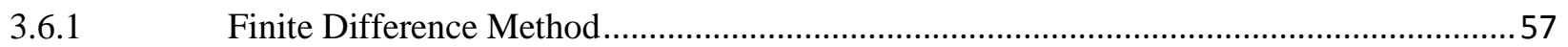

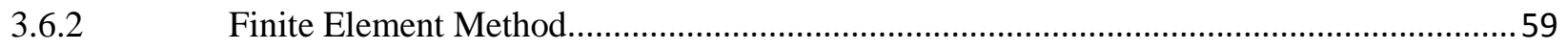

3.6.3 Comparison between Analytical and Numerical Results ..............................................61

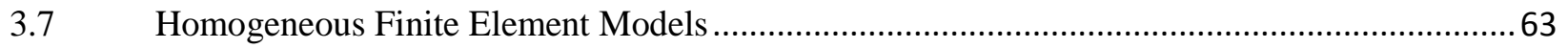

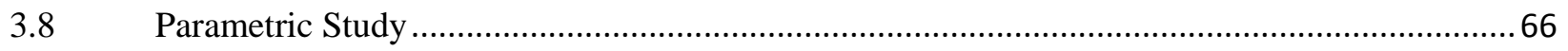

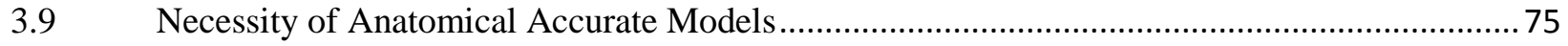

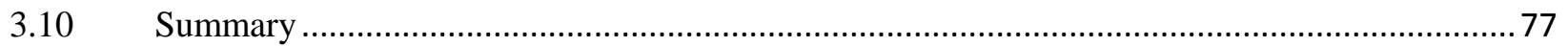

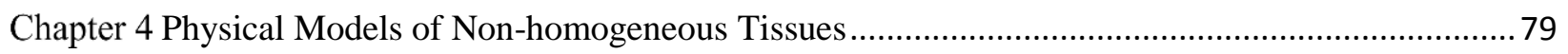

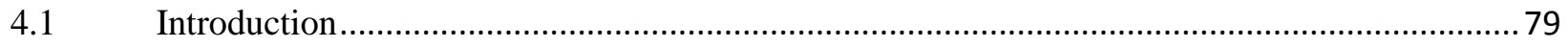

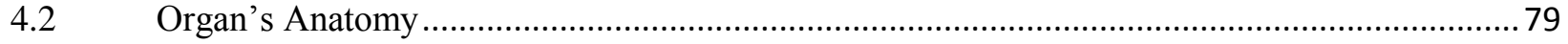

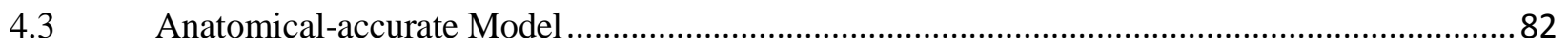

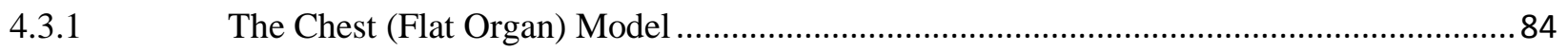

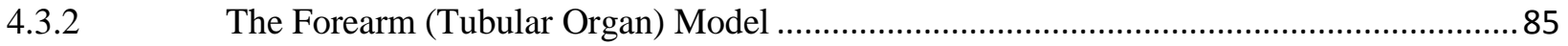

4.3.3 The Breast (Hemispherical Organ) Model .................................................................. 87

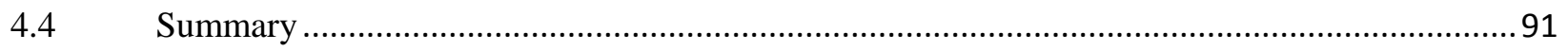

Chapter 5 Thermal Analysis of External Organs with Embedded Tumor ...............................................92

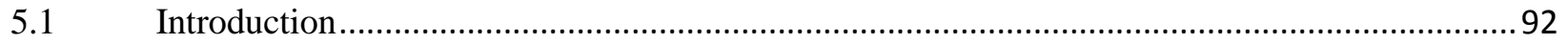

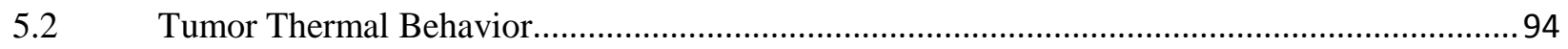

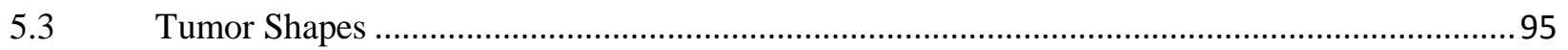

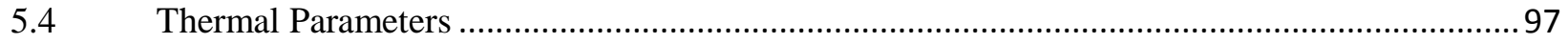

5.4.1 Blood Thermal Parameters: Density $(\rho b)$, Specific Heat (cb) and Perfusion Rate $(\omega b) \ldots . .98$

5.4.2 Thermal Parameters of tissues: Thermal Conductivity, Density and Specific Heat ........... 99

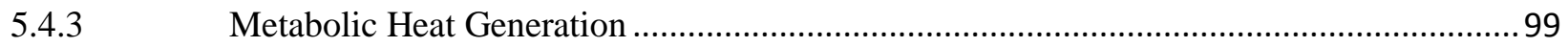

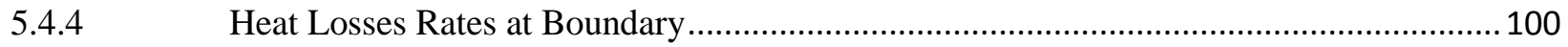

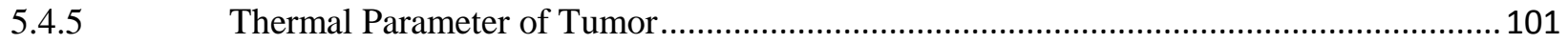

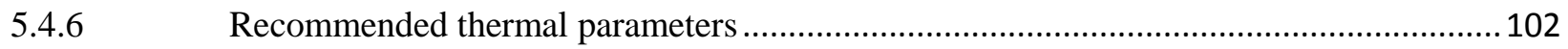

5.5 Thermal Analyzer: COMSOL Multiphysics ...................................................................... 103

5.5.1 Numeric Models (Gridding and Meshing) ................................................................. 104

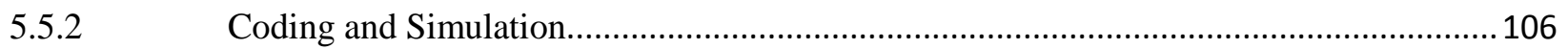




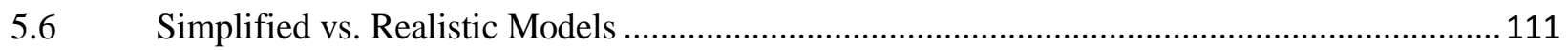

$5.7 \quad$ Necessity of Anatomic-accurate (Realistic) Models ............................................................. 115

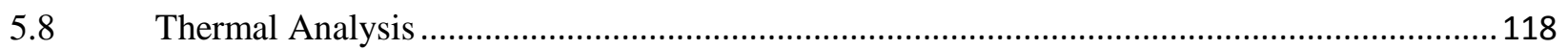

5.9 Effect of Physio-thermo-biological Parameters and the Surrounding ................................... 124

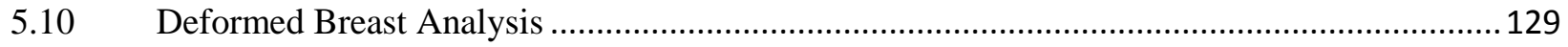

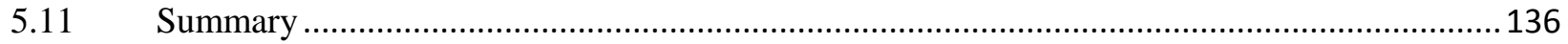

Chapter 6 Parameter Estimation —-Indirect Approach ................................................................... 137

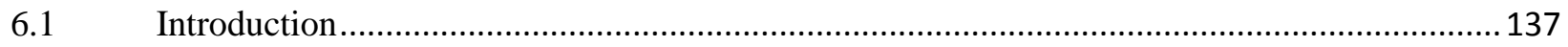

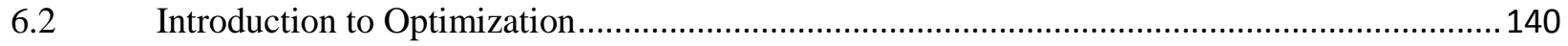

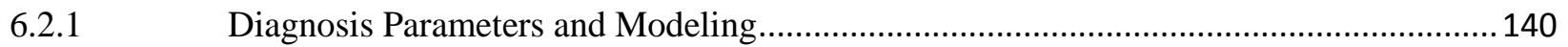

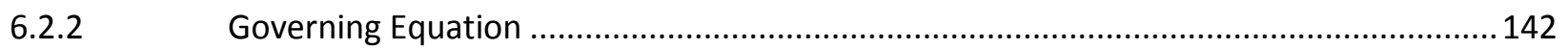

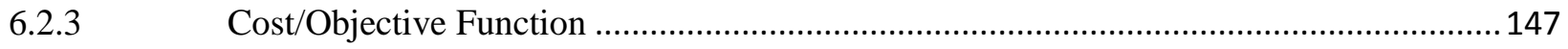

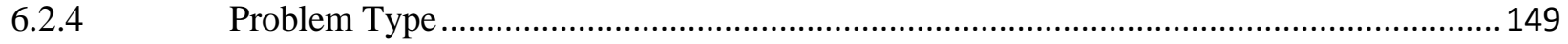

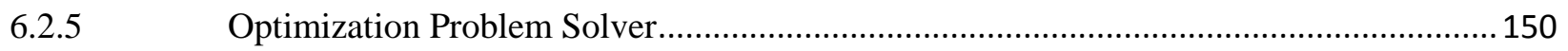

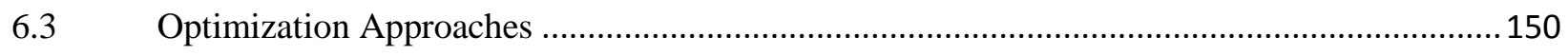

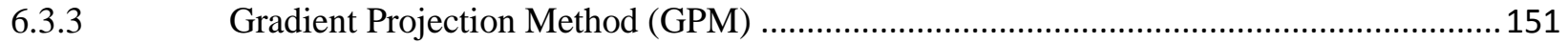

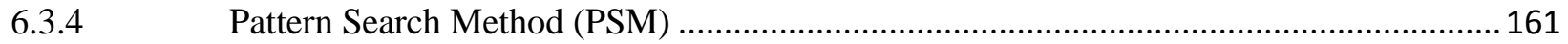

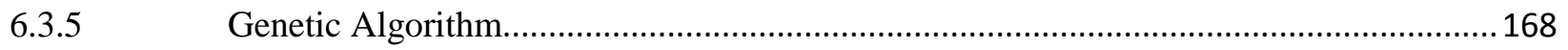

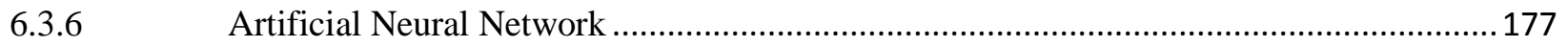

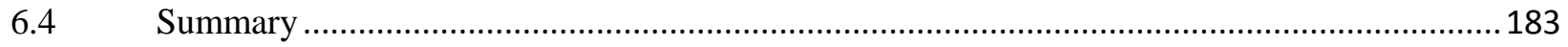

Chapter 7 General Conclusions and Future Work .......................................................................... 185

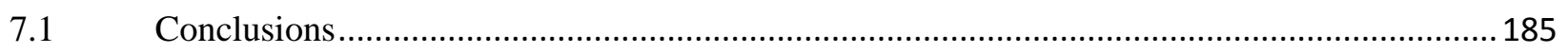

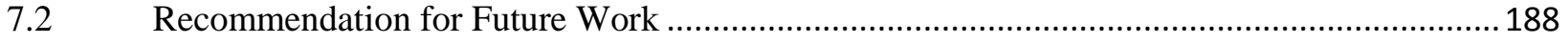

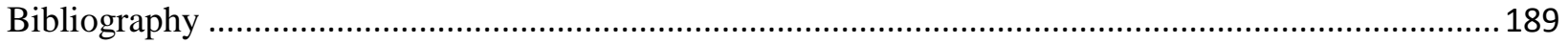

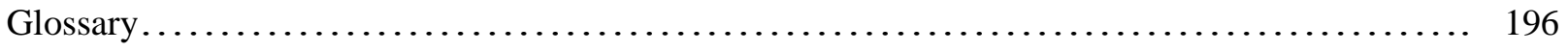




\section{List of Tables}

Table 2- 1 Study of Performance and Suitability Evaluation of Breast Thermogram..........................229

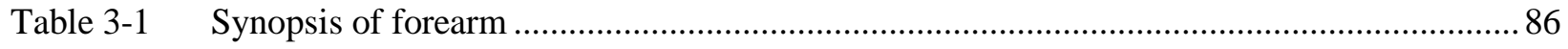

Table 5- 1 Thermal parameter of blood (assumed in previous studies) …..........................................98

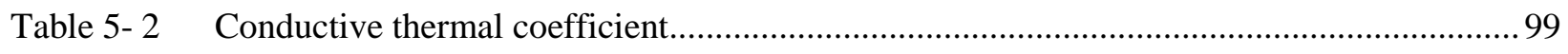

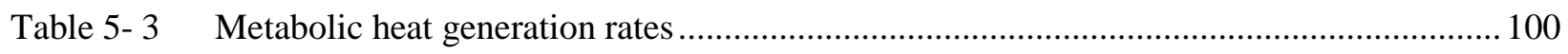

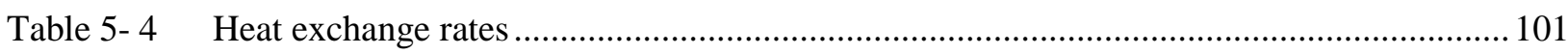

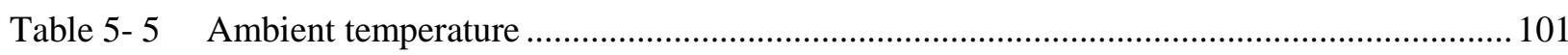

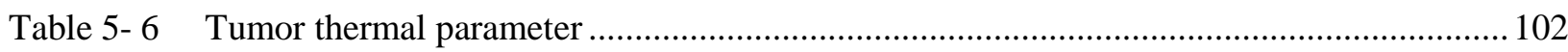

Table 5- 7 Thermal and biological parameters ............................................................................... 103

Table 6- 1 Estimated heat source parameters................................................................................ 161

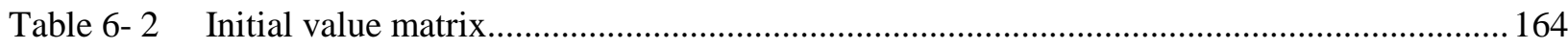

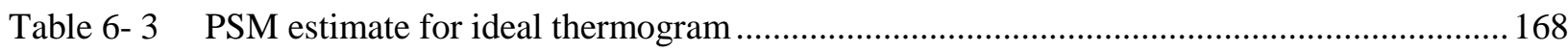

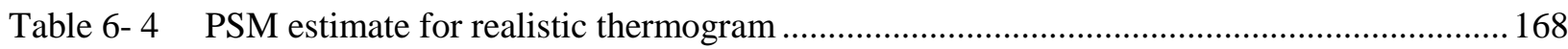

Table 6- 5 GA estimate for ideal thermogram of tumor buried in natural breast ................................ 176

Table 6- 6 GA estimate for realistic thermogram of tumor buried in deformed breast ....................... 176 


\section{List of Figures}

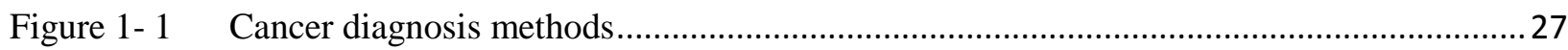

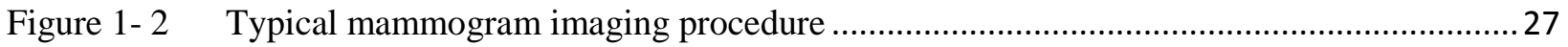

Figure 1-3 A patient lying on the scanning table outside the magnet.............................................2 27

Figure 1- 4 Thermogram detection of breast cancer ....................................................................... 27

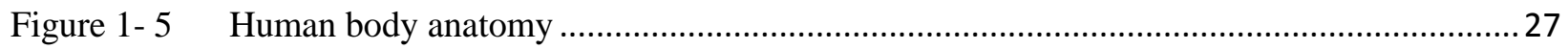

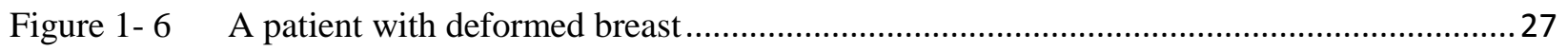

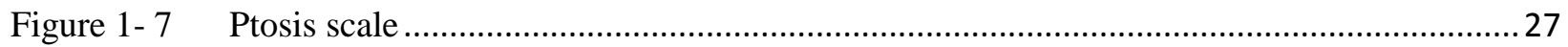

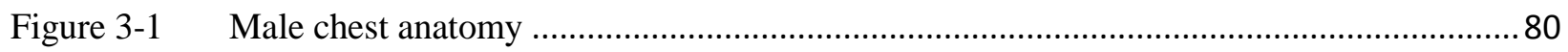

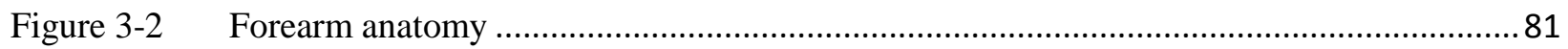

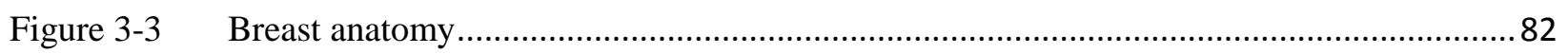

Figure 3-4 2D model of a female breast ............................................................................... 83

Figure 3-5 Male chest-(a) Cross-sectional diagram, and (b) Development of chest model base ........ 84

Figure 3-6 Three-dimensional model of male chest .................................................................. 85

Figure 3-7 Human forearm diagram - (a) Cross-sectional diagram, and (b) Development of forearm

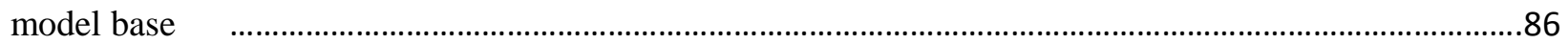

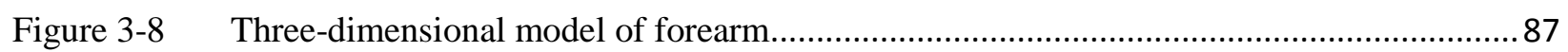

Figure 3-9 Breast diagram - (a) and (b) Physical dimensions, (c) cross-sectional diagram, and (d)

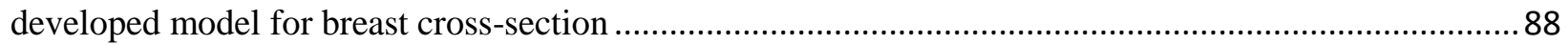

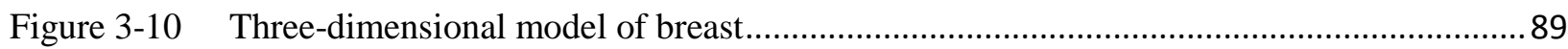

Figure 3-11 Deformed breast - (a) Mildly deformation due to standing posture and (b) Developed

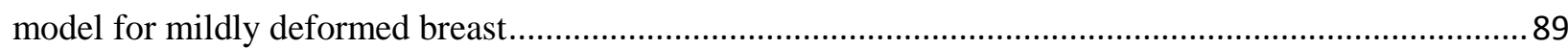

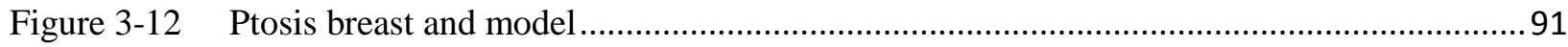

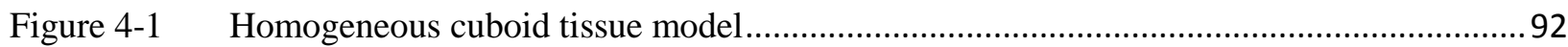

Figure 4-2 Homogeneous cylindrical tissue model ….................................................................... 92

Figure 4-3 Homogeneous spherical tissue model ..................................................................... 92

Figure 4-4 Radial temperature flow in homogeneous cuboid tissue from body core toward surface for

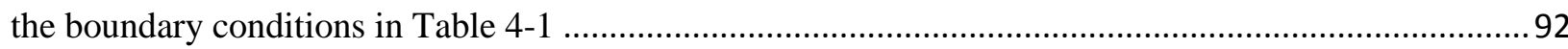

Figure 4-5 Radial temperature flow in homogeneous tubular tissue from body core toward surface for

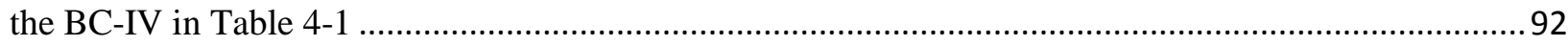

Figure 4-6 Radial temperature flow in homogeneous curved tissue from body core toward surface for

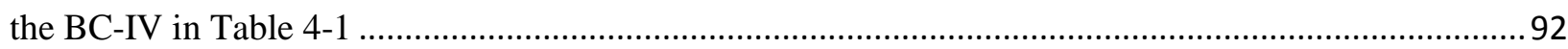

Figure 4-7 Comparison of radial temperature flow in homogeneous cuboid, tubular and curved tissues

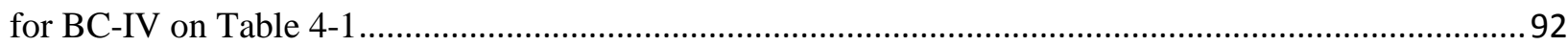

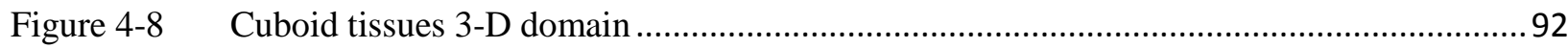

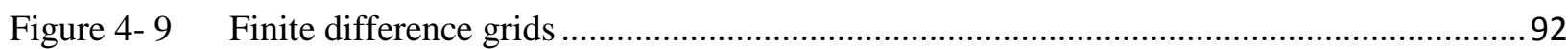

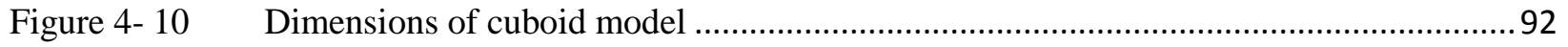

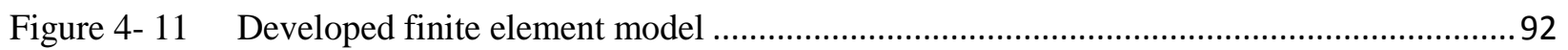

Figure 4- 12 Simulated radial temperature flow in homogeneous cuboid tissues using analytical, FDM

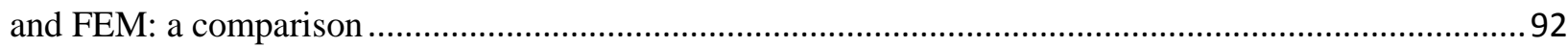

Figure 4-13 FEM mesh diagrams for (a) cuboid, (b) cylindrical and (c) spherical tissues...................92 
Figure 4-14 Temperature flow in radial direction estimated analytically and using FEM for three different shapes of organs

Figure 4-15 Analytical and FEM estimation of biological parameter's effect on radial temperature flow in cuboid tissues; influence of -(a) Qm, (b) k and (c) $\omega \mathrm{b}$..... 92

Figure 4-16 Analytical and FEM estimation of ambient and physical parameter's effect on radial temperature flow in cuboid tissues; influence of -(a) ha, (b) Te, and (c) Tissue thickness

Figure 4-17 Effect of biological parameter on temperature profile in cylindrical tissue -influence of (a) Qm, (b) $\mathrm{k}$ and (c) $\omega \mathrm{b}$

Figure 4-18 Effect of ambient conditions and model thickness on temperature distribution in cylindrical tissues; influence of(a) ha, (b) Te and (c) Tissue thickness....

Figure 4-19 Effect of biological parameter on temperature profile in spherical tissue -influence of (a) Qm, (b) k and (c) $\omega \mathrm{b}$

Figure 4-20 Effect of ambient and model thickness on temperature distribution in spherical tissues- (a) ha, (b) Te , and (c) Tissue thickness.

Figure 4-21 Estimation of radial temperature flow over anatomic-accurate (realistic) and simplistic (homogeneous) models for-(a) chest tissues, (b) forearm tissues, and (c) breast tissues .......................92

Figure 5- 1 Tumor shapes and margins .................................................................................. 96

Figure 5- 2 Cuboid tissue model 3D (not to scale) .................................................................... 104

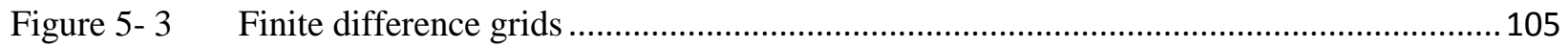

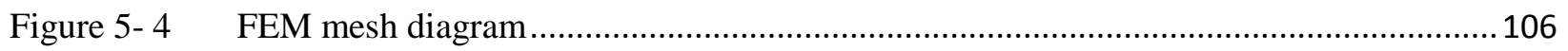

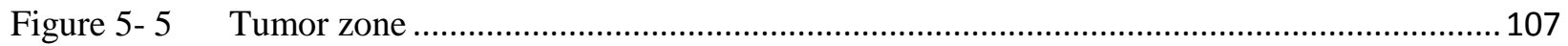

Figure 5- 6 Comparison of numerical analysis results; plot of-(a) radial temperature flow between core and skin through tumor center, and (b) surface temperature between side to side opposite points right above the tumor centre

Figure 5- 7 Comparison of numerical analysis results; for interior temperature distribution-(a) using FDM and (b) using FEM, on the horizontal plane through tumor center; and isothermal regions-(c) using FDM and (d) using FEM on the same plane 108

Figure 5- 8 Spatial temperature distribution over chest skin surface. 109

Figure 5- 9 Physical models - a) homogeneous, and b) anatomic-accurate for tumor in - (i) cuboid (chest), (ii) tubular (forearm), and (iii) hemispherical (breast) organ

Figure 5-10 FE structure of - a) homogeneous, and b) anatomic-accurate models for tumor in-(i) cuboid (chest), (ii) tubular (forearm), and (iii) hemispherical (breast) organ .....

Figure 5- 11 Comparison of temperature profiles obtained for a tumor in anatomic-accurate (realistic) and simplistic (homogeneous) chest model along the line-(a) between the body core and skin through tumor center, and (b) over the surface between two side to side opposite points right above the tumor center.

Figure 5- 12 Comparison of temperature profiles obtained for a tumor in anatomic-accurate (realistic) and simplistic (homogeneous) forearm model along the line - (a) between the body core and skin through tumor center, and (b) over the surface between two side to side opposite points right above the tumor center.

Figure 5- 13 Comparison of temperature profiles obtained for an on-axis tumor in anatomic-accurate (realistic) and simplistic (homogeneous) breast model along the line - (a) between the body core and nipple, and (b) over the surface between two side to side opposite points through the nipple. 117 
Figure 5- 14 Chest tumors; (a) to (d) surface temperature for tumor 1 to 4, respectively, (e) center-line

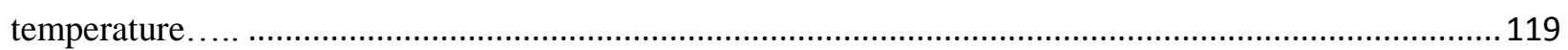

Figure 5-15 Area of temperature grow, (a) to (d) for chest tumor 1 to 4, respectively ...................... 120 Figure 5- 16 Forearm tumors, (a) to (d) surface temperature for tumor 1 to 4, respectively, (e) center-line

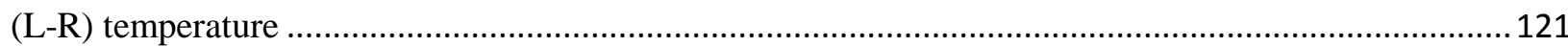

Figure 5- 17 Area of temperature grow, (a) to (d) for forearm tumor 1 to 4, respectively ................... 122

Figure 5- 18 Breast tumor analysis; (a) to (f) surface temperature for tumor 1 to 6 , respectively ....... 123

Figure 5- 19 Center-line temperature for tumor in undeformed breast............................................. 124

Figure 5- 20 Effect of bio-thermal parameters of tumor, e.g. influence of - (a) metabolism, (b) perfusion and (c) heat conduction rate of tumor on breast surface temperature

Figure 5-21 Effect of physical parameters of tumor, e.g. influence of-(a) location, and (b) diameter of

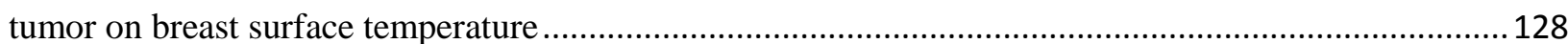

Figure 5- 22 Effect of environmental parameters, e.g. influence of - (a) heat exchange rate, and (b) ambient temperature of laboratory on breast surface temperature in case of a tumor ............................129

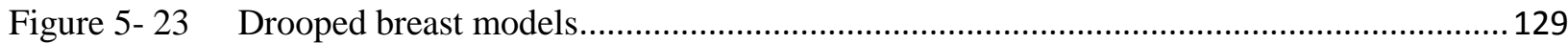

Figure 5- $24 \quad$ FEM models for drooped breasts .............................................................................. 130

Figure 5- 25 Mild deformed breast tumor thermal analyses - (a) to (f) for tumor 1 to 6, respectively 132

Figure 5- 26 Center-line temperature for mild deformed breast tumor along - (a) L-R (left to right side)

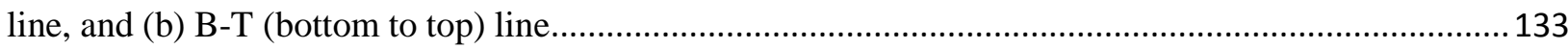

Figure 5- 27 Tumor in deformed breast, (a) to (d) surface temperature for tumor 1 to 4, respectively, (f)

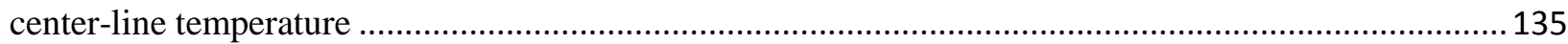

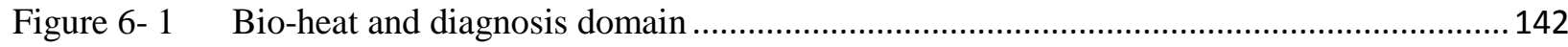

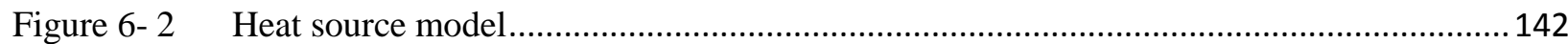

Figure 6- 3 Comparison of heat source model response with analytic result for various - (a) heat exchange rate, (b) ambient temperature, (c) metabolic heat and (d) thickness ...................................... 144

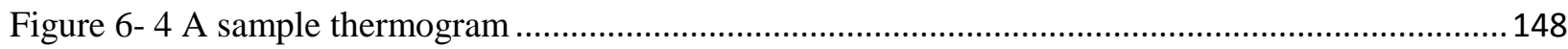

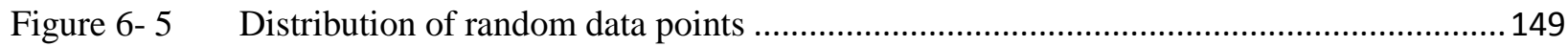

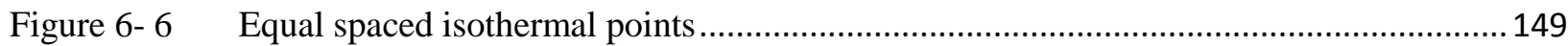

Figure 6- 7 Heat source model responses to $\mathrm{R}$ and respective slopes of $\mathrm{dT}$ (a)dR ............................ 154

Figure 6- 8 Heat source model responses to $h$ and slopes of dT(a)dh............................................. 155

Figure 6-9 Heat source model responses to Tc and slopes of dT(a)dTc ....................................... 155

Figure 6- 10 Representation of a chromosome ................................................................................ 171

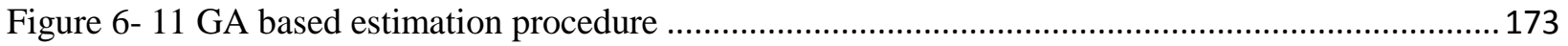

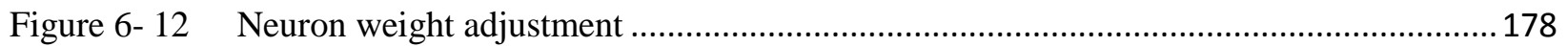

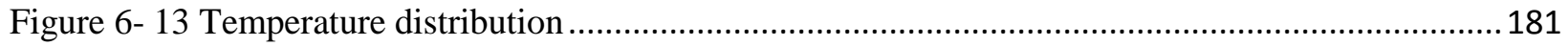

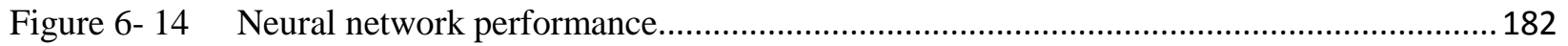

Figure 6- $15 \quad$ Best Simulated patterns .......................................................................................... 183

Figure 6- 16 Simulated pattern (not the best one)..................................................................... 183 


\section{Chapter 1 Introduction}

\subsection{Background}

Breast cancer has the second highest incidence (26\%) of all female cancer cases in Canada. More than $11 \%$ of Canadian women are expected to develop breast cancer during their lifetime (by the age of 90). An estimated 25,000 new incidences of breast cancer will be diagnosed and one in 5 (an estimated 5,000) Canadian women will die from breast cancer in 2015. These statistics remain unchanged over the past years [1]. However, breast cancer mortality rates have decreased by 42 percent since the peak in 1986. The significant decline in the breast cancer mortality rate suggests that there has been important progress made in cancer control which includes earlier detection through regular mammography screening, advances in screening technology, and improved treatments. In relation to breast cancer, advancements in earlier detection are an aspect of cancer control efforts that have been found particularly successful. When women are diagnosed at earlier stages of the disease, more treatment options are available, consequently providing a better chance at surviving the disease. Most recent data indicates a five-year survival rate for breast cancer of 88 percent, while in 1986, when breast cancer mortality was at its peak, the survival rate was 79 percent. Even small improvements in the early diagnosis and treatment of breast cancer would likely save thousands of lives annually.

At present, many imaging techniques such as X-rays, MRI (Magnetic Resonance Imaging) [2], Ultrasound [3], Mammogram [4], SPECT (Single Photon Emission Tomography), Positron Emission Tomography (PET) [5], Thermography (microwave and infrared) [6] and others clinical examinations are used for medical diagnosis of breast cancer. X-ray mammography and clinical breast examination (CBE) are the current gold standards of breast cancer screening. Ultrasound and MRI are also used as secondary screening tools to elucidate suspicious findings from the Xray mammogram. Non-optical imaging techniques including PET, electrical impedance tomography (EIT), and thermal imaging either rely on specific intrinsic characteristics of breast tissue or employ exogenous tracers/contrast agents [2] to image the breast or to identify lesions within it, therefore, amongst them some are useful in screening while others are useful only in diagnosis. As a whole, the above methods together have established success in cancer screening and diagnosis. However, each method has its own advantages and limitations. For example, X- 
Ray mammography represents the current standard of care and has contributed to a significant reduction in breast cancer mortality $[1,2]$. The sensitivity of X-ray mammography appears to be greater than $80 \%$ in women over 50 and less for younger women, there is still a need to detect cancers earlier for treatment. Furthermore, mammographic specificity is known to be limited [4] because it is inherently incapable of direct observation of physiological information relevant to the "functioning of cancer," which ultimately limits the specificity and prognostic value of X-ray mammography.

Moreover, methods that measure changes in functional processes, as opposed to those that measure structural changes, show the greatest promise for future improvements [7, 8, 9]. Functional MRI and PET do assess function, but their high cost and invasiveness have prevented their widespread deployment in breast screening and diagnosis. Likewise, Ultrasound, Mammography and MRI look at the structure or anatomy of the human body but modality of Infrared (IR) Thermography is assessing of body function as a heat or lack of heat signature due to physiological or metabolic change $[10,11]$. Therefore, achieving an accurate functional representation of the tissues in any living organism without disturbing and damaging, which is a goal of medical professionals, scientists and engineers for centuries, could be achieved from IR technique. Consequently, thermography, a rather physiological tool, can indicate developing disease states earlier than anatomical examination (computed tomography (CT) imaging or X-rays, etc.). Other advantages of thermography include high portability and real time imaging, which make it possible for the data to be recorded in computers for further processing. The imager converts the thermal energy to electrical signals in order to display the temperature profile of the subject, which contains a number of colors indicating different temperatures. However, the thermal imager should be used in an indoor environment where external factors, such as ambient temperature, humidity, and electrical sources, can be controlled.

Furthermore, the important distinguishing feature among the imaging techniques is, some are "invasive" and "ionizing" and some are "non-invasive" and "nonionizing". Unlike X-ray, MRI and Mammography; the Infrared (IR) Thermogram technique is non-contact, comfortable, and safe and has no radiation dose and can be applied repeatedly on the human body without known risk of morbidity.

IR is a diagnostic tool that relies on the human body's inherent quality as an Electromagnetic (EM) radiator, since it emits energy in the infrared range as a function of skin temperature 
distribution. Abnormalities in local body skin surface temperature have been recognized as a sign of disease for centuries, much before humans knew about the cause of ailments or of pain. Using hands to assess body temperature was primitive practice but information underlying abnormalities of skin temperature was not revealed properly. Today's sensitive temperature measuring devices invite physicians to identify subtle local thermal abnormalities and find an association between thermal image and a certain disorder.

Body surface temperature abnormalities have been recognized as a sign of disease for centuries, much before humans knew about the cause of ailments or of pain. An abnormality in local body temperature indicates a general sign of some disease under the region where the local surface temperature is higher or lower. But only some primitive practices (i.e. using hand or thermometer) were observed to assess body temperature. For that reason, information underlying abnormalities of skin temperature was not revealed properly until the modern sensitive temperature measuring devices became available. The modern IR camera or sensor can accurately measure the spontaneous Electromagnetic (EM) energy in IR frequency that is radiated from the human body as a function of skin temperature. The skin temperature, an important live character, is emanating from the heat discharge by cells activities and blood perfusion. Variation in skin temperature may be up to $0.5^{\circ} \mathrm{C}$ for a practically healthy body whereas for a defective body it may increase up to $5^{\circ} \mathrm{C}[12,13]$. Researchers discovered that local body surface temperature is controlled by metabolism, blood circulation underneath the skin, and heat exchange between the skin and its environment $[14,15]$. Change in any of these parameters could influence temperature distribution and heat flux at skin surface, and thus reflect the physiological state of the human body. The small change in surface temperature information in medical diagnosis and clinical therapy is a measure of such conditions as neuromuscular injury, breast cancer, rheumatism, arthritis-caused bone joint inflammation, lunge oncogenes, and thyroid gland disorders. The inflammation, metabolic heat rate, interstitial hypertension, abnormal vessel morphology and lack of response to homeostatic signals are some of the certain features that make tumors, in particular, behave differently than normal tissues in terms of heat production and dissipation [16, 17]. Therefore, abnormal skin temperature profile can be used to predict the location, size and thermal parameters of the hyperactive region as well as follow up the treatment procedure $[14,18]$. 


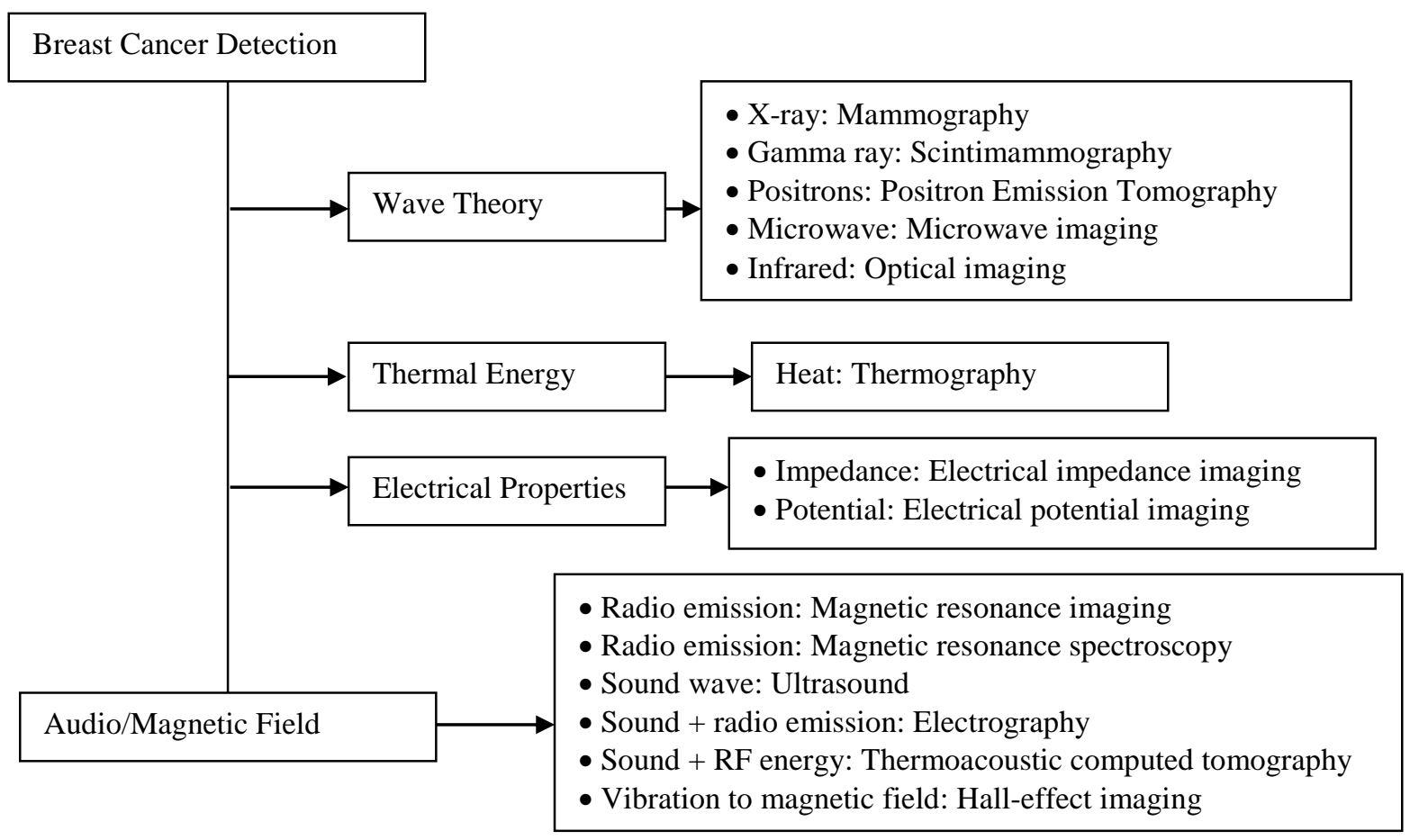

Figure 1-1 Cancer diagnosis methods [22]

The local skin temperature over a tumor is significantly higher than the normal breast because of convection properties related with increased blood perfusion, heat conduction, and metabolism around the tumor. Lawson [12] was the first to recommend the application of thermography for breast cancer detection, when he observed that the local skin surface temperatures above a tumor were significantly higher (approximately $2^{\circ} \mathrm{C}-3^{\circ} \mathrm{C}$ ) than the normal skin temperatures. Lawson and Chughtai [13] established that the regional temperature abnormality over an embedded tumor was because of the convection effects linked with the increased metabolism around the tumor, and the increased blood perfusion. Advance aspect of IR imaging techniques and detection methods of breast cancer from such images are discussed in detail by Diakides and Bronzino, [6]. In [19] Santa Cruz et al., after making a comparative investigation between thermography and boron neutron capture therapy (BNCT), concluded that the thermography, a potential imager of tissue functionalities, can help to locate abnormally high temperature regions as well as melanoma nodules that are virtually invisible in CT images.

As discussed, IR imaging has already paved its way as a promising adjunct screening tool and recent advances in the IR technology further promoting its medical application as a noninvasive tool for imaging tissue functionalities and the influence of vascular, neurogenic and 
metabolic processes that affect them. A high accuracy data acquisition system is currently available, but a big challenge is in deriving a solid correlation between superficial surface temperature and physiological bio-thermal parameters of a tumor that is yet to be resolved [20]. Numerous efforts have utilized the modern IR technology to predict the location, size and thermal parameters of tumors from the abnormal temperature profiles during the last few decades which included anatomical modeling or analogous modeling and inverse solution approach.

\subsection{Medical Imaging Technology}

Breast cancer grows in the epithelial cells, which are found along the terminal duct lobule unit. Abnormal growth of epithelial cells can lead to the formation of a tumor. If the growth is restricted, the cancer is classified as benign. If the growth is rapid or has the ability to progress to other regions of the body, it is classified as malignant or carcinoma. More often than not, a malignant breast is firm and irregular in shape. Various imaging modalities are extensively applied in breast cancer now-a-days. However, confirmation can only be made after mammography and clinical examination (biopsy).

Currently, available methods for cancer (emphasis is given on the screening of breast cancer) detection is summaries in Figure 1- 8. It can be seen that these methods are based on the underlying principles of wave theory, heat energy, audio/magnetic field and electric properties. A comparative study among Mammography, MRI, Ultrasound (US) and Thermogram is discussed in the following sections while applied to breast cancer screening.

\subsubsection{Mammogram}

In mammography, a 2D image of the breast is formed by passing X-rays through the breast. Absorption of X-ray photons varies between tissues, so the gray-level of a pixel in a mammogram gives an indication of the proportion of different tissues in the column of the breast that the X-rays corresponding to that pixel passed through. Therefore a mammogram is a projected 2D image of the breast, rather than a true 2D image of a slice of the breast (compared with an MRI, which will be discussed shortly).

Where no absorption of X-ray photons has occurred (such as outside the breast) the mammogram appears black, and where total absorption has occurred it will appear white. Fibroglandular tissue is highly X-ray absorbent, whereas fat is not, so the fibroglandular region of 


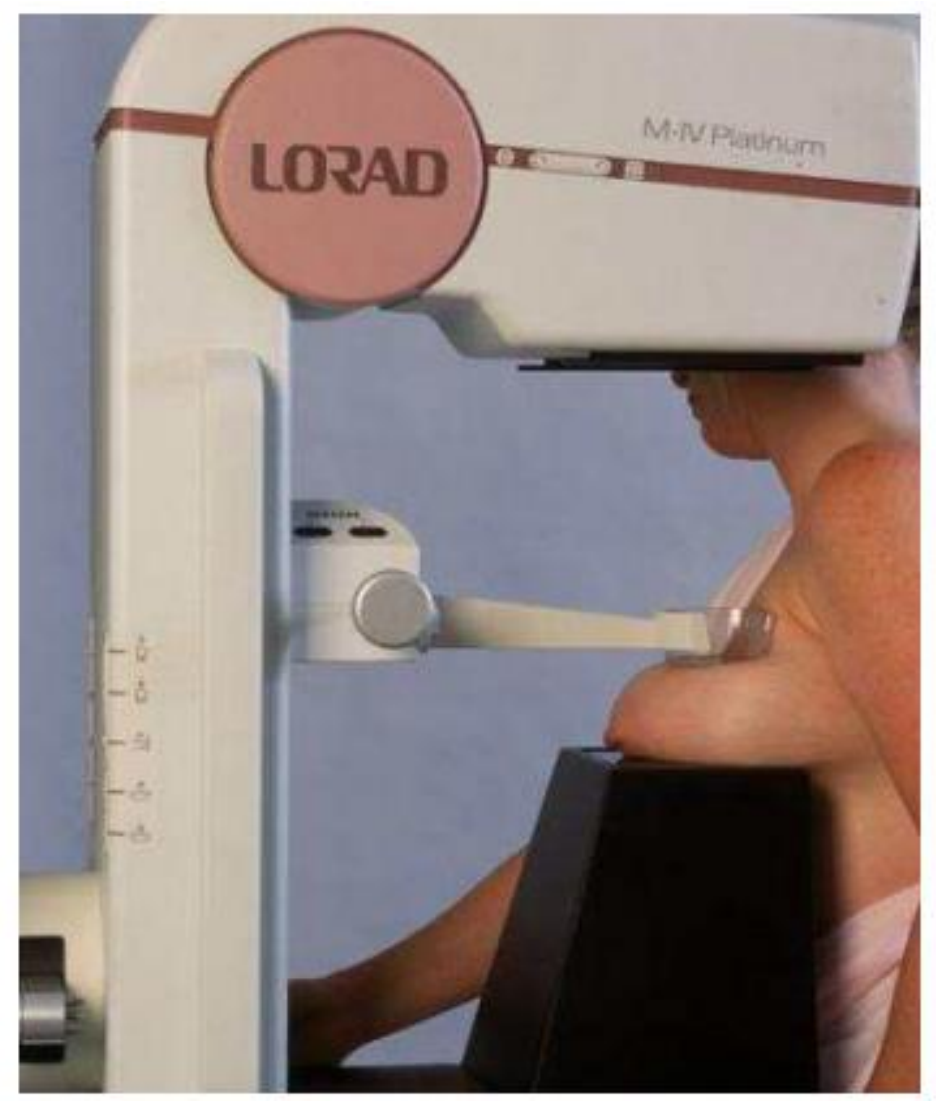

Figure 1-2 Cranio-caudal (typical) mammogram imaging procedure [29]. Compression causes deformation of breast tissues substantially.

the breast will appear brighter in mammograms. Because of this, mammography as a method of screening is only performed on older women, younger women tend to have dense breasts with a lot of fibroglandular tissue, and X-ray images of their breasts can sometimes turn out to be completely white. Typically, the lower age limit chosen for screening programs is about 50 years, an estimate for the onset of menopause. After menopause fibroglandular tissue is replaced by fatty tissue, resulting in better contrast mammograms.

For women for whom good quality mammograms can be taken, useful information can be gathered using mammography. Mammograms have the highest resolution of the three imaging modalities, and the small-scale structure of the breast can be visualized. In particular, this means that micro-calcification can sometimes be spotted. These are small deposits of calcium salts that are often the earliest indicators of the presence of a tumor. If un-obscured, they appear in X-ray images as small regions of very high contrast. 
There are various angles from which the X-ray image can be taken, the most common being cranio-caudal (CC), where the direction of the X-ray photon travel is head-to-toe, and mediolateral oblique (MLO), which is shoulder-to-opposite-hip. Less common views are mediolateral (ML) (center of the chest outwards to the side), and latero-medial (LM) (the opposite direction to ML). Screening mammography typically involves two views of the breast, usually CC and MLO. The use of a combination of views allows the radiologist to study the breast anatomy from different angles and come to a more informed judgment.

When mammograms are taken the breast is compressed heavily by two plates with normal in the direction on the X-ray photon travel. For example, in CC mammography, the breast is compressed in the vertical direction. Compression is carried out for two reasons: firstly, it spreads out the tissue, allowing the radiologist to see breast structures more clearly, and secondly, it reduces the distance between X-ray source and receptor, so that a smaller dose can be used.

Typical imaging procedure for CC mammography is shown in Figure 1- 9. Note how the amount of compression is quite substantial, and how the compressed breast shape is very different to the non-deformed shape.

\subsubsection{Magnetic Resonance Imaging (MRI)}

The magnetic resonance phenomenon was discovered independently by Edward Purcell and Felix Bloch in 1946, and resulted in the pair sharing a Nobel Prize in 1952. Atoms with nuclei with odd numbers of protons and neutrons were known to, in the presence of a static magnetic field, to tilt at a certain angle and precess at a certain speed; they discovered that the precession will intensify under an electromagnetic field at a precise frequency, known as the resonance frequency. The response to the field varies between atoms and is affected by other atoms in the same molecule, which can be exploited to obtain structural information on a molecule. These discoveries were originally put to use in analytic chemistry and biochemistry, in studies determining the composition of chemical compounds, and was known as nuclear magnetic resonance (NMR) spectroscopy. In the 1970s it was discovered that with the inclusion of a weak gradient magnetic field, together with the strong magnetic and electromagnetic fields, the MR phenomenon could provide spatial information, which eventually led to its use as a means for noninvasive tissue imaging. Clinical use began in the 1980s, for which the procedure was renamed magnetic resonance imaging, the word 'nuclear' being dropped because of its obvious negative connotations. 
The MR machine is a large machine with a cylindrical hole to contain the patient. For breast MR imaging, the patient lies prone (face down) on the scanning table, with the breasts hanging pendulously in depressions that contain the breast coils (the equipment used to detect the resonance signal). The table is moved into the MR machine, which houses the magnet, and imaging can then takes place, a procedure which can last 10 to 30 minutes. 2D images can be taken of different 'slices' of the breast, and a 3D picture obtained. The images can be taken in any desired plane. Figure 1- 10 displays the MR machine and the position of the patient.

There are two methods which can be used to search for tumors. One is to simply search for suspicious masses in the images, by eye or using image analysis algorithms. The second is a technique that can be used to search for vascular tumors. If a contrast-enhancing agent (a chemical with significantly different MR properties to normal tissue, which appears very bright in the images) is injected into the blood and MR images taken at different times, blood vessels will appear to get lighter and darker as blood density (and thus contrast-enhancing agent density) varies. Similarly, masses with their own blood supply, such as vascular tumors, will also change intensity. Vascular tumors often show up extremely clearly in contrast-enhanced MRI.

The main obvious advantage of MR scans is that they provide three-dimensional data on the breast. Each image is a true 2D image of that slice, not a projected 3D onto 2D image as with mammograms. It can be used equally effectively for younger (pre-menopausal) women as with older women. However, the resolution is a lot lower than mammograms and structures such as micro-calcification cannot be resolved. There are also practical disadvantages: MR imaging is expensive, and there are relatively few MRI machines in the world. Also, the process can be mildly unpleasant, and due to the huge magnetic fields involved, not possible on patients with, for example, pacemakers or metallic implants. Since the imaging procedure takes some time, MR images are highly affected by blurring and other motion-related artefacts, motion due to both chest movement for breathing and shifts in position by the patient. 


\subsubsection{Ultrasound}

Ultrasound imaging is performed with the breast in another new position to that of MRI or mammography. Here, the patient sits up, with the breast falling to their side. The ultrasound probe is pushed gently against the breast and dragged along the skin.

Ultrasound is the least useful of the three imaging techniques as ultrasound images are very poor quality, and is not suitable as a screening tool. It can be used as an alternative method for premenopausal women if MR imaging is not possible. However, there is one advantage unique to ultrasound: since the probe is pressed against the skin, the effect of different levels of compression on the breast can be seen. Tumors are generally much harder than surrounding tissue, and a method of identifying tumors is to look for masses that retain their shape under compression. Benign masses tend to be softer, so ultrasound is a useful diagnostic tool.

\subsubsection{Infrared Thermogram}

Thermogram was originally developed for military purposes. In the past fifty years the commercial and industrial applications of thermogram are incredible. Recent applications have extended to engineering applications and medical imaging. At present, thermogram is used in condition monitoring, medical imaging, infrared mammography, veterinary medicine, night vision, chemical imaging, volcanology, non-destructive testing, fault finding (in buildings), surveillance (in security, law enforcement and defence), and so on. Airport officials are using

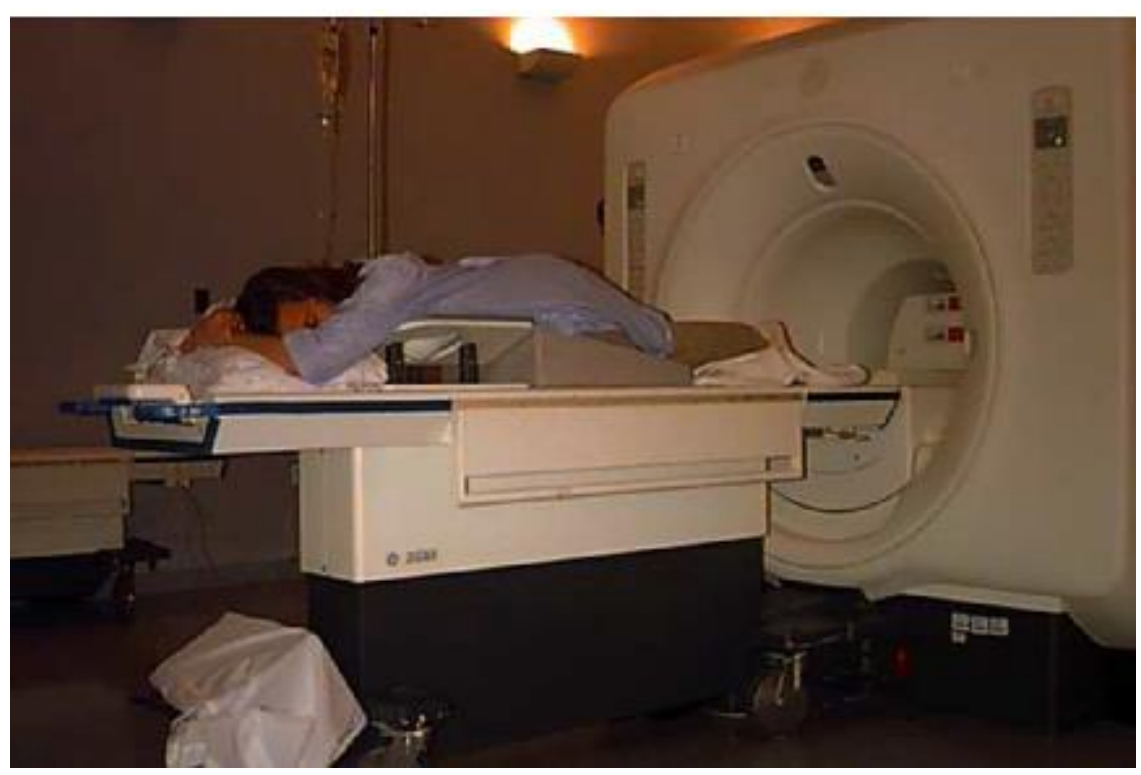

Figure 1-3 A patient lying on the scanning table outside the magnet before taking MRI [47] 
thermography for detecting suspected swine flu cases during the 2009 pandemic. Firefighters use this technology to see through smoke, to locate persons, and to determine the base of a fire. Maintenance technicians also utilize the tools to locate overheating junctions and segments of power lines, which are a tell-tale sign of forthcoming failure. For indicating the heat leaks in faulty thermal insulation, the building construction technicians usually see thermal signatures and use the IR images to improve the efficiency of heating and air-conditioning units. Some physiological changes in warm-blooded animals including the human being can also be monitored with thermal imaging during clinical diagnostics. IR thermography makes use of a thermal imager to detect the IR radiation and measure the heat pattern of the object surface or human skin [21, 22, 23]. Being noncontract, non-invasive, hygienic, and highly informative the promising diagnostic tool is currently used with X-rays, MRI, Ultrasound, CT, SPECT, PET and others.

Human skin emits infrared radiation as a function of body temperature according to the Stefan-Boltzmann law. Thermal energy is continuously generated within a human body due to the metabolic process and the status of the blood circulation system; hence the vascular system will be reflected on thermogram as the area of specific temperature pattern. According to the blackbody radiation law, invisible radiation in the infrared range of the electromagnetic spectrum is emitted by all objects near room temperature. Thermal imaging cameras can detect the radiation and produce visible images, called thermogram. The spectrum and amount of emitted thermal radiation is a function of temperature; therefore, thermography is capable to show temperature variations in objects having above absolute zero background temperature and hence can passively see all objects regardless of ambient light. The pattern is presented on a black and white or color image of temperature distribution. From this image decision can be made in medical application by (i) absolute temperature, (ii) temperature fixture i.e. shape of abnormal temperature zone and (iii) temperature asymmetry between the left and right side of a patient's body. The temperature asymmetry between the left and right side of the human body gives an indication of abnormality.

When a natural temperature difference is present between the object and ambient (background), the passive themography can be used. On the other hand, in active thermography, an external energy source is required to produce a thermal contrast between the feature of interest and the background. The basic energy expression in IR thermogram includes the incident energy (the energy profile viewed through a thermal imaging camera) which is the sum of the emitted energy (the actual informative energy of what is intended to be measured), the transmitted energy 
(that passes through the subject from a remote thermal source), and the reflected energy (that reflects off the surface of the object from a remote thermal source). A thermal imaging camera is capable of performing algorithms to interpret all these three forms of energy data and then employs a series of mathematical algorithms to build a picture in the viewer and record a visible picture, usually in JPG format.

Thermography makes a significant contribution to the evaluation of patients suspected of having breast cancer. The obviously abnormal thermogram carries with it a high risk of cancer. A report summarizes the results of patients with questionable or stage Th-III (thermogram risk level 3) thermograms where approximately 58,000 patients, most of whom had breast complaints, examined between August 1965 and June 1977, a group of 1,245 women were diagnosed at initial examination as either normal or benign disease by conventional means, including physical examination, mammography, ultrasonography, and fine needle aspiration or biopsy, when indicated, but nevertheless categorized as stage Th-III indicating a questionable thermal anomaly. Within five years, more than a third of the group had histologically confirmed cancers. The more rapidly growing lesions with shorter doubling times usually show progressive thermographic abnormalities consistent with the increased metabolic heat production associated with such cancers. Thermography is useful not only as a predictor of risk factor for cancer but also to assess the more rapidly growing neoplasms. 


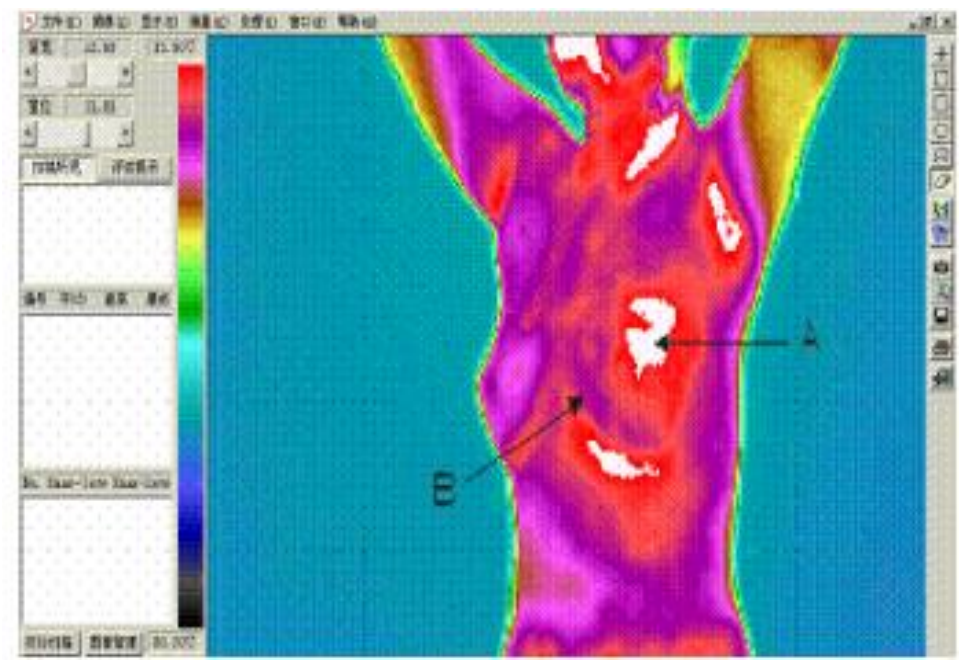

Figure 1-4 Thermal distribution for a benign lump in left breast detected by thermography [42]

An abnormal breast thermogram of a 44-year-old patient is shown in Figure 1- 11. This patient had been diagnosed with a benign lump in her left breast using Doppler Ultrasound. From her breast thermography, it can be observed that she has a relative warm area in her upper-outer quadrant (arrow A) and a relatively cool area in her lower-inner quadrant (arrow B). These indicate that the tumor is embedded in upper-outer quadrant of breast.

\subsection{Challenges of the Available Systems}

At present, Mammography is the most reliable technique for early detection of breast cancer. The patient discomfort associated with compression of the breast tissues (Figure 1- 9 Typical mammogram imaging procedure), exposure to ionizing radiation, and frequent false positive readings are the major limitations. MRI has major limitations associated with cost and availability. Ultrasound, although non-invasive, has a relatively low detection rate and has a low sensitivity for ductal microcalcifications in the breast. According to [3], breast ultrasound is low cost and radiation free and can be useful if combined with family history, but it is not recommended as a standalone technique for evaluating the presence of breast cancer.

Having drawbacks of the techniques above mentioned, thermography has been investigated as an alternative method to detect abnormalities, which could be attributed to breast cancer. Thermography acquires superficial temperature data and then analyzes it to reveal the concealed tissue functionalities. Being incapable to expose anatomical structures, its application is limited 
to as a screening tool adjunctive to other techniques. Thus, in physiological test, it alone is not sufficient for medical practitioners to make a diagnosis. However, when used adjunctively with other laboratory and outcome assessment tools, such as anatomical techniques including mammography, ultrasound and CT scanning, thermography may contribute to the best possible evaluation of breast health. The International Academy of Clinical Thermology issues the following position announcement: "The proper role of thermography is not as a replacement for mammography". Therefore the current accepted role of thermography is for applying only in breast cancer screening and detection.

Followings are the reasons behind:

- There is no one test that can have above $99 \%$ accuracy for detecting cancers. Therefore, no single test available that can be used alone as an adequate screening or detection tools for breast cancer.

- A physiological imaging procedure (thermography) cannot replace the existing anatomical imaging procedure (e.g. the mammography). Because the two approaches look for completely different pathological processes.

- Thermography is far more sensitive than mammography especially for slow growing non-aggressive cancers that may not be detected by mammography.

Breast thermography is a complementary screening and detection procedure, which when added to a woman's breast health examination substantially increases the sensitivity in detecting pathologies associated with the breast. As a unique physiological examination procedure, breast thermography is the only known test that can also serve as an early warning system by identifying women who have high-risk pre-cancerous infrared imaging markers. The procedure can also play a role in prognosis and as a method of assisting in monitoring the effects of treatment.

Evolutionary methods are being applied to pin point embedded tumor's physical and thermal parameters with surface temperature. The process is still ongoing and the insubstantial studies present some theoretical prediction of spherical tumor in soft tissues of an un-deformed breast. In reality tumor shape and thermal behaviour is completely unknown and breasts might undergo elastic deformation due to sagging or drooping. In addition, the analysis also requires an accurate anatomical model of some other organs except breast which differ exclusively in person to person. 
Deference may be in tissue heterogeneity, layer dimension, patient age, heredity, etc. and all of them have important influences on the thermal behaviour.

\subsection{Computational Tools and Estimation Methodologies in Health Care}

In clinical application, the health related problems have to be treated for healing in a fast and objective fashion. However, clinical observations taken by technologists might be influenced by internal (coming from the technologist) as well as external (independent from the technologist) impacts. The objectivity of classification is subject to the receptivity of human senses which are adversely influenced by the experiences or level of training, psychological conditions (tiredness, hurry, stress, etc.), as well as ambient conditions (lighting, destructive noise, humidity, etc.). The failure in awareness questions the entire recognition procedure. The recognition processes itself, which is subject to the above mentioned conditions, may cause a slowdown and/or lead to an erroneous diagnosis.

Computerized approaches for detecting clinical problems have become critically important in healthcare [24]. Mathematical analysis, modeling, and computer simulation become standard tools underpinning the current progress in developing the computational tools. A radical change in technologies have been integrated to develop a system for addressing the core of medicine, including patient care in ambulatory and in-patient setting, disease prevention, health promotion, rehabilitation, home care and so on. Computational tools are becoming a widespread use for the support of patient medical diagnosis and treatment, the assessment of the quality of care, and the improvement of decision making, modeling, simulation, and medical research. A computerized support in the analysis of patient information and implementation of a computer-aided diagnosis and treatment systems increase the objectivity of the analysis as well as speed up the response to pathological changes.

\subsubsection{Anatomic-accurate Physical Model}

The thermal texture computations in tissue interior as well as on the skin surface of a particular organ involve formulating the bio-heat transfer problem exactly, which can be achieved by addressing the problem on an anatomically accurate model. Such realistic models should include the tissue heterogeneity, organ's anatomy etc. Anatomical structures of outer organs, wholly or partially, can roughly be given some standard shapes. Inspecting Figure 1- 12, the 
superficial view of human anatomy, it is understood that human organs or segments has merely a flat, tubular or hemispherical outlook. In particular, the flat surfaces such as the dorsum (back) and the chest (male) segments can be assumed as rectangular boxes. Likewise the tubular organs such as arm, forearm and legs can be assumed as solid cylinders. Similarly, the curved organs like breast and buttock can be modeled as hemispheres.

The heat transfer problems in biological tissues address a typical second order differential equation and, therefore, the analytic computations require considering an appropriate coordinate system consistent with the organ's geometry. For a complete understanding of the thermal features of the human body the study deals with three different organs - the chest, the forearm and the

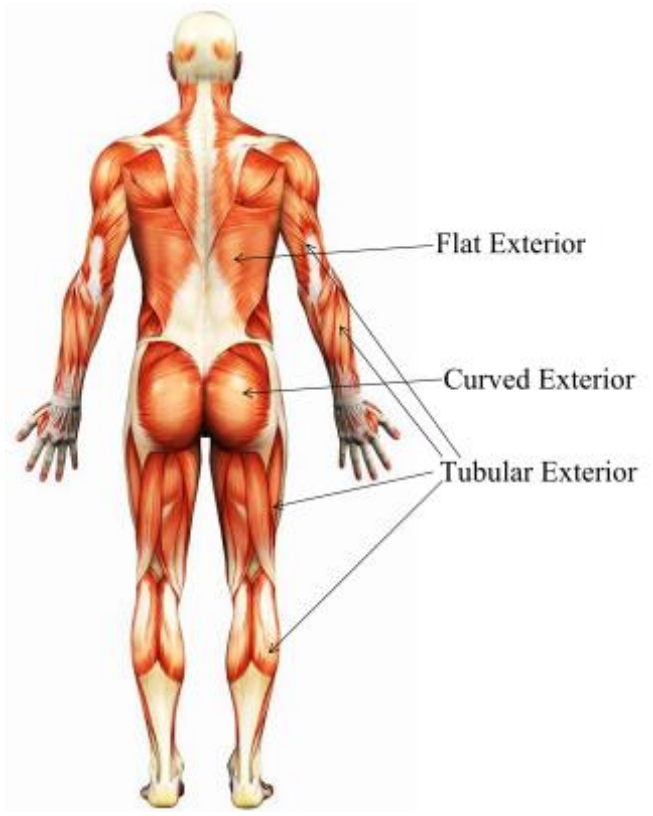

Figure 1-5 Human body anatomy (external view) [81]

breast.

\subsubsection{Deformed Organ Model}

Apart to the organ's geometry, the proportion to the constituent tissue layers might vary due to age, body mass index (BMI), gender etc. and the variation should be properly introduced while modeling. In particular, breasts deform widely due to body posture changes and/or ptosis disorders. Therefore, they evoke the necessity of considering the phenomenon of gravitational or structural deformations of breasts for accurate modeling. Following is the brief reasoning: 


\subsubsection{Deformation in Supine-to-Standing-to-Crawling Posture}

The simplest example of appropriateness of the model is considering breast deformation due to the supine-to-standing-to-crawling posture. The indirect approach is often an ill-posed problem and the solutions are highly sensitive to the changes in body posture. When IR images are taken of a patient, they are generally carried out with the patient standing, with the breast nipple hanging down under gravity. The thermo-physical solutions estimated in such posture would be different from the supine and/or crawling positions. An acceptable accuracy can be achieved only if the deformations are modeled properly.

In addition, during surgery (in particularly during a lumpectomy procedure, where a tumor is removed by the surgeon), or during open biopsy (where a sample of a lump is removed by the surgeon for examination by a pathologist) the patient will be in the supine position. A surgeon

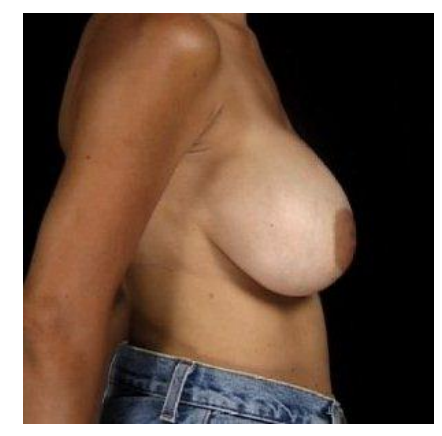

Figure 1- 6 A female patient with deformed breast [86]

using IR images as a guide will have to use some degree of guesswork when locating the tumor, which hinders minimally-invasive surgery. A deformable model of the breast could be used as guide for the surgeon: the model would be built from the original IR images, and the direction of gravity reversed to simulate the breast during surgery.

Similarly, the model can be used as a guide during fine-needle or core biopsy (where a small sample of the tumor is removed for examination using a needle, a thin needle in the case of fine needle biopsy and a larger needle in core biopsy). Needle biopsy is a very accurate test provided enough correct material is removed, but since the biopsy has to be guided by the images, it is not always easy to get a good sample. A model of the breast could be used as a guide during biopsy, and in fact if the model is highly accurate it could be used to automate the biopsy procedure. 


\subsubsection{Deformation in Ptosis Patients}

Ptosis of the breast is a natural consequence of aging where the term 'ptosis' is used in medical for referring to the drooping or sagging of breasts. The rates and the degree of ptosis depend on many factors, such as smoking, number of pregnancies, breast size before pregnancy, body mass index (BMI) and so on. The deformation process is also influenced by patient's heredity, which includes the elasticity of skin tissues, breast size, weight gain and the influence of weight gain in increasing the breast size, and the ratio to the adipose and glandular tissues. Unlike the simple hemispherical model for a natural breast, the deformed ptosis breast, shown in Figure 1- 15 , requires taking into account the multilateral deformations of tissues.

Furthermore, the severity of ptosis, shown in Figure 1- 16, is evaluated by the position of the nipple relative to the infra-mammary fold (the point at which the underside of the breasts attached to the chest wall), which could tremendously affect the accuracy of the model, and consequently the inverse thermal solutions.

\subsubsection{Indirect Problem Solvers}

The estimation methodology involving an indirect approach to determine unknown thermophysical or geometrical parameters of a tumor region using the temperature profiles (may be obtained by infrared thermography) on the skin surface necessitates minimizing a cost function. The generalized cost function can be given as:

$$
\min || T_{o}-T_{m}(x)||
$$

Subject to $x \subset\{$ List of characteristic parametets $\} \in \Omega$, where $\Omega$ is the region over which the solution is bounded, $T_{o}$ is the actual temperature pattern over the skin surface that may be obtained from thermogram (using infrared camera, numerical simulation etc) and $T_{m}(x)$ is the estimated temperature pattern that is obtained from the model expression (or directly from the

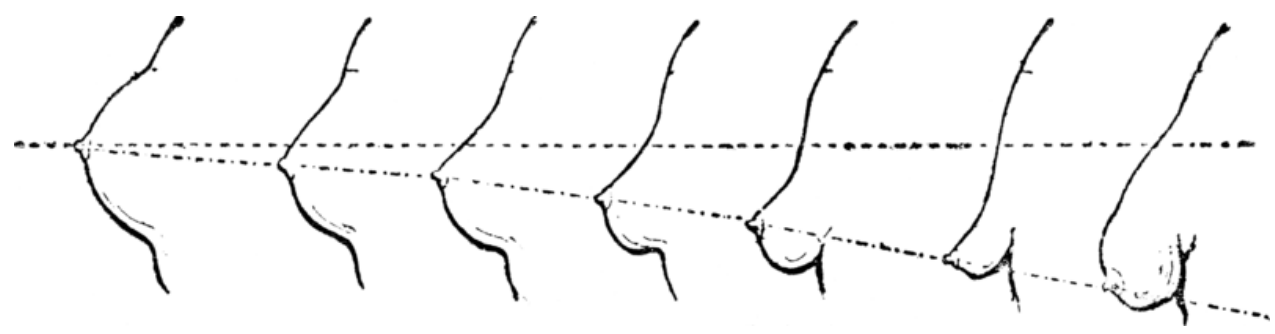

Figure 1-7 Scaling of breast ptosis disorder, starting from mild (left) to sever (right) [89] 
numerical models). Several evolutionary methods — such as the static slope method, pattern search method, genetic algorithms and artificial neural network method are commonly used in optimization problems.

The static slope estimation approach is developed on the basis of a straight-forward temperature expression of local skin experiencing an abnormal thermal behaviour due to an embedded tumor. The abnormal increase in spatial local skin temperature is expressed as a function of tumor parameters by analyzing the contribution of some important thermo-physical parameters to the skin temperature and developing a model for employing to the optimization technique. The solution matrix comes up with a number of local and/or the global minima from which the best solution is sorted using some physical constraints and clinical knowledge. The pattern search estimation methodology uses a diagonal matrix along which the optimization process follows. The convergence rates and step sizes can be pre-determined or computed using the adaptive algorithm.

Unlike the previous two approaches the Genetic Algorithm (GA) uses a solution pool to estimate tumor parameters. This non-gradient optimization tool can be applied for the characterization of wide range tumors ranging deep-seated to shallow deep and malignant to benign. The GA would run several times with different random initial population, even though the final estimates would be identical regardless the initial population.

Finally, an evolutionary optimization method, the neural network consists of a set of highly interconnected processing elements (neurons). The neurons are connected through a set of connection weights, or synaptic weights. Every neuron $i$ has $M_{i}$ inputs, and one output $y_{i}$. The inputs are real valued quantities labeled $s_{i 1}, s_{i 2}, \ldots \ldots, s_{i M i}$, representing signals coming either from other neurons in the network, or from the external world. Every neuron $i$ has $M_{i}$ synaptic weights, each one associated with each of the neuron inputs. The synaptic weights are labelled as $w_{i 1}, w_{i 2}, \ldots \ldots, w_{i M i}$, and represent real valued quantities that multiply the corresponding input signal. Every neuron $i$ has also an extra input, which is set to a fixed value $I_{i}$, and is referred to as the threshold of the neuron. Every neuron computes its own internal state, or total activation, according to the following expression:

$$
x_{i}=\sum_{j=1}^{M_{i}} w_{i j} s_{i j}+I_{i}=\sum_{j=0}^{M_{i}} w_{i j} s_{i j}
$$


Every neuron $i$ computes its output according to a function $y_{i}=f\left(x_{i}\right)$, where $f$ is the neuron function or transfer function. A three layer feedforward neural network with backpropagation learning/training algorithm will be applied for addressing the inverse problem.

\subsection{Motivation and Overview}

Having looked at the different imaging techniques in section Chapter 1 and Chapter 1, it can be seen that infrared thermogram provides superficial information as an indication of tissue functionalities in terms of heat radiation and dissipation. It is obvious that due to the lateral symmetry of the human body the thermal distribution over skin surface of a healthy organ differs from the other symmetrical organ. The subtle thermal information can be associated with the thermal and physical parameters of an underlying tumor. In order to relate the behavioural feature of temperature changes with hyperactive tissue functionalities, a realistic model of the human organ is required [25]. These models are regarded as a second reader, it is notable that the final decision is left to the medical professional. For several regions, the development of numerical models are regarded as an extremely challenging task. First, more importantly, the imaging system may have serious imperfections. Tissue compression, ionizing radiation, cost, availability, and rate of false positive (or false-negative) detection are the common deficiencies of the available clinically approved imaging modalities. Second, the image analysis task is compounded by the large variability in the appearance of abnormal regions. Sharp boundary discontinuity between cancer and healthy tissues in terms of heat generation and dissipation is debatable. Third, asymmetric thermal contrast for an organ's physical deformations that may vary with patient's age, body mass index (BMI), non-uniform thickness of fat layer etc, in particular, thermal asymmetry due to gravity induced deformation associated with body posture and ptosis disorders in breast tissues incur complications improving the sensitivity and the specificity. Finally, abnormal regions are often hidden in dense tissue. Relative lower specificity is a challenge for early detection of deep-seated benign.

The inverse thermal problems are not well-posed because the solutions may not be unique. Deducing characteristics parameters from the superficial thermal data is highly sensitive to the parameters and in many cases lead to a NP (Non-deterministic Polynomial-time)-hard problem. Essential measures namely the accurate correlation between the thermal-physical parameters and 
the temperature features, the realistic physical and thermal models, minimizing unknowns etc. attribute reasonable accuracy to the forecasted results.

According to the brief discussion, it's very crucial for determining the physical and thermal parameters from the external temperature data viz. thermograms. The analysis requires the establishment of a forward thermal model and anatomically accurate physical model for shallowly and deeply buried tumors either in malignant and benign stages, besides, creating a powerful algorithm for the inverse problem setting for the detection and characterization of the hyperactive region.

\subsection{Thesis Contribution}

This research has developed a novel methodology for correlating surface temperatures with thermal, biological and physical parameters of tumors. The methodology has been devised based on both thermal and evolutionary algorithm based analyses for parameterizing hyperactive tissue. The study has developed anatomically accurate physical models of human body organs; performed a theoretical analysis of thermal behaviour of the organs; estimated size, depth, perfusion rate and metabolic rate of spherical tumors buried in the physical models; and validated the results with simulated and experimental data.

The theoretical study begins with finding the internal temperature distribution in the tissue interior by solving steady sate Pennes' bio-heat equation analytically with different boundary conditions. The bio-heat equation is solved in three coordinate systems (because the external organs of the human body have flat, tubular and curved outlook, in general) and the influences of thermal conductivity, metabolism, blood perfusion and heat exchange rate are studied for homogeneous and isotropic tissues models. The purpose of this analysis is to promote the validation for numerical simulation results.

Physical models have been developed for chest, forearm and breast, with considering the geometrical and behavioural variation of tissues found in different layers of the above organs as well as considering the bio-thermo-physical variations in embedded tumors or cancer tissues, if any. Such models are called anatomic-accurate or realistic physical models. Bio-heat transfer problems on the realistic models are then solved numerically to estimate thermal behaviour for healthy as well as for cancerous tissues. Assuming a uniform bio-thermal property within an entire 
organ, an approximate model has also been developed, such model is called homogeneous or simplistic model, and the bio-heat transfer problem has been addressed both analytically and numerically. The purpose of the homogeneous model is to validate the accuracy of the developed model and to establish the numerical method as a stand-alone estimator. In addition, physical deformation in the female breast has been considered while developing the realistic models. In general, the female breast deforms due to body posture change (because of gravitational force) and for ptosis. Both phenomena have also been considered in preparing the deformed breast models and their thermal behavior have been analyzed accordingly. Discrepancies in estimated thermal patterns observed on realistic models with and without tumors have been utilized for screening, for making the decision whether there is a tumor or not. Further, a correlation between surface temperature distribution over the model and the underlying tumor has been established. Successful investigation in utilizing the correlation between the skin temperature and physiological parameters has turned the screening tool into a useful diagnostic tool.

Optimization methods, such as, the gradient projection method (GPM), Genetic Algorithm, Artificial Neural Network (ANN), and pattern search method have been used for inverse solving of thermal problems. Optimization algorithms have been tailored for each method to estimate tumor physio-bio-thermal parameters like the thermal conductivity, metabolic rate, blood perfusion rate, size and depth. In GPM, the search moves along the projection of the temperature function, requires a continuous heat flow equation derived analytically. However, the analytic solution can provide radial temperature flow inside tissues only but the main goal of the thesis is estimating tumor parameters from surface thermal data. Therefore, the analytic result has been reproduced by an equivalent heat source model (performance of the model discussed in section 6.3.1.1) and then the heat-source model governing equation is employed to estimate the surface pattern. Another purpose of the heat-source model is transforming the bio-thermal parameters into diagnosis parameter (discussed in section 6.3). The GPM algorithm computes the governing function and the gradient of the function to be minimized, an initial guess and a structure option containing some non-default options required by the process. The process also needs to pass in some constraints that confine the solution domain in a valid region. This function implements a two-metric projection method for optimization with upper and lower bounds on the values of the variables. The process has used a quasi-Newton strategy, where limited-memory BroydenFletcher-Goldfarb-Shannoalgorithm (L-BFGS) updates are used in computing the step direction, 
and a backtracking line search is used to find a step satisfying an Armijo condition along the projection arc. The algorithm has also tailored to change the number of L-BFGS corrections to store for the quasi-Newton update, and whether or not to use skipping or damping of the L-BFGS updates. Using this function also requires specifying the vectors LB and UB giving the lower/upper bounds on the variables. The elements of LB/UB have been set between 1 and 2 after normalizing the variables.

The GPM requires partial derivatives of the temperature function to the optimization variables which necessitates a continuous differentiable governing equation that has been met with the proposed heat source model. However, the heat-source model has been derived from the analytic results obtained on simplistic (homogenous) models. The optimization problems on realistic models require solving the bio-thermal problem numerically which is a desecrate process and unable to compute the gradients. Furthermore, determination of the projection arc (the direction at which the optimization search takes place) is not easy. The PSM can be applied to optimize a non-differentiable function while the optimum point search takes place in every direction. The method includes computing the basis matrix, direction matrix, exploratory moves and updating parameters and step sizes. The main challenges of this process are the computation time as the search takes place in every possible direction and the process does not guarantee the global minima rather it can easily converge toward a local minimum depending on the assumption of the initial search point. The study has suggested an initial search vector that might lead to the global minima, however, the proposed search vector depends extremely on comprehending the thermogram sensibly. Moreover, applying evolutionary optimization techniques can be a useful tool for finding the optima. Therefore, the PSM estimated values can be taken as an initial parameter vector for further accuracy. Application of the biological model onto optimization task in form of evolutionary strategies appears as very effective in situations where standard analytical approaches fail to succeed (either because the solution could not be analytically grasped and described or simply the exhaustive calculations became infeasible). Therefore, the study has also addressed the optimization problem with GA and ANN.

In GA optimization, the process includes defining an objective function that has to be minimized with the above mentioned five optimization variables, defining the stopping criteria, setting up GA parameters, generation of initial population (encoding) and ranking them, iteration through generation while finding pair and mate, performing mating using single point cross-over 
and mutation, re-evaluating the newly generated population and rank them, repeating the process and finally displaying the outcome (decoding). The objective function is designed to determine the absolute difference between the spatial temperature dataset acquired by thermography to the numerical simulation of heat flow problem over an anatomic-accurate (realistic) model (model that is developed while taking into account every significant tissue layer in a particular organ and outer structure of the organ). The numerically simulated temperature dataset is repeatedly computed for different values of optimization variables. The optimizing process will continue for maximum optimization error $1^{-5}$ (average absolute difference between target and simulated patterns) or for 100 generations. After a few trail, the process is run for 16 initial populations, mutation rate 0.15 and $50 \%$ selection rate. The optimization parameters are encoded to binary values where each parameter is a byte length and chromosome size is 50 bits. Random number generator function is used to create initial population and these populations are applied to evaluate the cost function and ranked, and then divide into two groups of parents. Parents are selected randomly from each group who will come forth to give production of new generation. The new generation (offspring) is created by a single point crossing where the crossing point is randomly chosen. Some offspring will undergo mutation process by toggling bit(s) i.e. replacing ' 0 's by ' 1 's and vice versa, the toggling bit(s) are also randomly selected. This entire newly generated offspring is decoded and applied to evaluate the function and determine the cost and sorted according to the minimum cost. This process is repeated until any stopping criteria are met. Finally the optimized parameters are denormalized and displayed with their respective fitness error. Though the GA process can compute any NP-hard, non-gradient problem with a good accuracy but the process does not always guarantee global minima and requires a huge computation time and memory. In addition, the test temperature pattern obtained from solving bio-heat flow problems on anatomic models numerical can be a significant source of error if the model is not developed accurately and the development of anatomic-accurate models is a challenge. However, the optimization process can also be performed without having a realistic physical model with the help of a trained artificial neural network (ANN).

The study has developed a 3-layer feed-forward ANN with back propagation learning to estimate thermo-physical parameters of tumors from surface thermal images. The network is developed with the MATLAB Neural Network Toolbox using the "newff" function. The "transig" transfer function was chosen for the hidden layers, "purelin" for the output layer and "trainlm" for 
back propagation training. The ANN is trained with idealized surface temperature profiles obtained for 5 different cases. Set of 500 data from the half of the symmetric pattern is normalized and then taken as training/validating input, $T(1 \times 500$ matrix). The target matrix $P$ is also normalized and each parameter is encoded with random numbers to produce 500 different values and scaled down to produce a $4 \times 500$ matrix. The learned network is then simulated for the above sample temperature pattern. The simulated output is, finally, decoded and justified with some constraints to filter out the noisy data and the algebraic mean of the remaining data is computed and treated as the output of that run. The forecasted parameter's values are applied to solve the bio-heat transfer problem on the developed anatomic-accurate model to produce the ideal temperature pattern (simulated). If such obtained temperature pattern is close (minimum average absolute error between the simulated and target pattern is $0.1 \%$ ) to the target pattern then the run is considered as a good run. In the end, the average of 20 good runs is computed to forecast the tumor parameters.

\subsection{Outline of the Thesis}

Chapter 2 is the literature review of thermogram assessment on living tissues, with a brief description of thermogram applied in breast tumor screening in particular. Studies, performed before 2000, had investigated the efficacy of this technology for early cancer detection in terms of Sensitivity (False-negative rate) and Specificity (False-positive rate). Continuing assessments of the capabilities in breast tumor diagnosis are ongoing. Recent studies have been using thermal information for correlating the temperature image with underlying physiological changes. However, clinical acceptance of such studies is still being argued. The chapter summarises major studies and their shortcomings relating to thermogram.

The direct solution of bio-heat problems in homogeneous tissues is obtained analytically in Chapters 3 and such tissues are also modeled to re-compute their thermal features numerically, where both approaches determine the interior thermal behaviour at a steady state with solving Pennes' bio-heat equation for nonlinear boundary conditions. Both approaches exhibit consistency in obtaining tissue interior thermal features.

Chapter 4 begins by providing an introduction to the human body anatomy with categorized organs into solid rectangular box, cylinder, and hemisphere and the imaging modalities applied 
especially to the breast, as well as motivating the need for models considering the organ's geometry as well as a deformable breast model.

In Chapter 5, the numerical approach for solving thermal features on organs with implanted tumors is discussed. The step-by-step procedure includes the development of structural models of organs mimicking their outlooks and heterogeneity, attributing the model layers with accurate physical, thermal, and biological parameters, meshing and solving the thermal problems for various conditions. The deformation of breasts is substantial due to aging, body posture change and ptosis. Therefore, this chapter also covers the problem which may arise immediately when the deformation of the breast is tackled, which includes modeling of the drooped breast, and investigating their thermal pattern and association with an underlying tumor.

Chapter 6 presents the optimization approaches to estimate the location, size and metabolic heat production rate of an embedded heat source. Different estimation methods are considered for example the Genetic Algorithm (GA), the Artificial Neural Network (ANN) and the pattern search algorithm. Full custom optimization algorithms are tailored according to the special feature of the thermal problem for each optimization process. The comparison of estimated parameters is presented. Finally a combination of GA and pattern search method is recommended.

General conclusions and future work is presented in Chapter 7.

\subsection{Summary}

With embedded tumor, an organ undergoes anatomical and physiological changes and between these two processes, one pathologic and the other physiologic, the latter one can be traced much sooner before the former. The medical imaging techniques such as mammogram, ultrasound, MRI, etc. inspect the pathologic (anatomic) changes whereas the thermography looks at the physiologic (functional) changes. This chapter presents a historical background of the imaging techniques with their own limitations and advantages. Though the anatomic changes provide a clear indication of a hyperactive (abnormal or defective) region but such changes might be detected at advance stages and consequently reduce the survival rate. For reducing the mortality rate by facilitating with more treatment options, it requires earlier detection by inspecting the functional changes in tumor tissues. An estimation methodology (based on data analysis and modeling) has been recommended in this research to locate a tumor and estimate its size and thermal behaviour 
using abnormal surface temperature profiles, an obvious indication of functional discrepancies, may be obtained by IR thermography. The methodology could pave a way in developing an epochmaking medical imaging technology. To summarize, the improvement of IR thermogram involve in the advancement in the medical technologies, infrared technologies and computer multimedia technologies. 


\section{Chapter 2 Literature Review}

\subsection{Introduction}

Thermography measures body heat that is constantly radiating away from the surface of the skin. The skin is an organ that breathes, exchanges gases with the environment, cools us as well as keeps us warm. This process is called thermoregulation and is done by a part of the nervous system called the sympathetic system that regulates blood flow in the skin. The energy radiates in the infrared frequency range and the relation between radiated energy and temperature can be defined by Stefan-Boltzmann law stating that the total radiation emitted by an object is directly proportional to the object's area and emissivity and the fourth power of its absolute temperature. Since the emissivity of human skin is extremely high, measurement of infrared radiation emitted by the skin can be converted directly into accurate temperature values.

Empirical evidence shows that underlying cancer alters regional skin surface temperatures due to increased vascular flow and metabolism. Infrared imaging of organs with implanted tumors may have critical prognostic significance since it may correlate with a variety of pathological prognostic features such as tumor size, tumor grade, lymph node status and markers of tumor growth. Thus, because of its inherent ability to detect subtle, vascular and physiological changes, thermography has been promoted as an early cancer detection test where thermal analysis has been presented to the patient as a method of determining the actual risk of malignancy. This information can then be used to direct laser therapy, massage, and chiropractic care and can be processed further to diagnosis a hyperactive region. In this chapter the contemporary state of art of the IR thermogram technology is addressed.

\subsection{Background}

Since 480 B.C. continued research and clinical observations proved that certain human body surface certain temperatures are, indeed, indicative of normal and abnormal physiologic processes [26]. Infrared monitoring systems for night vision ushered in a new era in thermal diagnostics in the mid-2000s. In 1957, a discovery by R. Lawson promoted thermography diagnostic when he observed the skin temperature over a cancer in the breast was higher than that of normal tissue [12]. The empirical evidence that underlying breast cancer alters regional skin surface temperatures was investigated, consequently, in 1963 Lawson and Chughtai, two McGill 
University surgeons, published an elegant intra-operative study demonstrating that the increase in regional skin surface temperature associated with breast cancer was related to venous convection [13]. Numerous medical centers and independent clinics have started using thermogram for a variety of diagnostic purposes since the late 1970's, primarily, the "Department of Health Education and Welfare" and the "Food and Drug Administration" approved and classified the thermography as an adjunctive diagnostic screening procedure for the detection of breast cancer in 1972 and 1982, respectively. Based on the early quantitative experiment in [27], JF Head et al. [8] remarked that IR imaging of the breast may have critical predictive significance since it may correlate with a variety of pathologic prognostic features such as tumor size, tumor grade, lymph node status and the superficial surface temperature. The pathologic basis for these infrared findings, however, is uncertain. Guidi and Schnitt observed that angiogenesis is an early event in the development of breast cancer and may occur before tumor cells acquire the ability to invade the surrounding tissues and even before there is morphologic evidence of an in-situ carcinoma [28]. Gamagami studied angiogenesis by infrared imaging and reported that hypervascularity and hyperthermia could be shown in $86 \%$ of hidden breast cancers. He also noted that in $15 \%$ of these cases infrared imaging helped to detect cancers that were not visible on mammography [29]. The biomedical engineering evidence of thermography's value, both in model in-vitro and clinically invivo studies of various tissue growths, normal and neoplastic, has been established [10, 30]. Thermography evaluation has two obvious viewpoints: the sensitivity of thermograms taken preoperatively in patients with known breast carcinoma, and the incidence of normal and abnormal thermograms in asymptomatic populations (specificity) and the presence or absence of carcinoma in each of these groups.

Performance and suitability of thermogram for breast cancer detection had been studied in the last century which has been presented in the following

Table 2-1. The performance evaluation includes the sensitivity (false negative rate) and the specificity (false positive rate) of detecting cancers. Researches also revealed that the thermogram can be applied to early detection of cancers and consequently increase the survival rates. 
Table 2-1 Study of Performance and Suitability Evaluation of Breast Thermogram

\begin{tabular}{|c|c|c|c|}
\hline Subject size & Methodology & Findings and Remarks & Ref \\
\hline 4,000 women & Thermography & $\begin{array}{l}\text { Sensitivity of } 94 \% \\
\text { False-positive rate } 6 \%\end{array}$ & [31] \\
\hline $\begin{array}{l}4,621 \text { women } \\
(35 \% \text { under } 35 \\
\text { years) }\end{array}$ & $\begin{array}{l}\text { Thermogram on } \\
\text { asymptomatic } \\
\text { women }\end{array}$ & $\begin{array}{l}24 \text { cancers }(7.6 \text { cancers per } 1,000) \\
\text { Sensitivity of } 98.3 \% \\
\text { Specificity } 93.5 \%\end{array}$ & [32] \\
\hline 61,000 women & $\begin{array}{l}\text { Thermography on } \\
\text { abnormal breast } \\
\text { persistently for } 10 \\
\text { years }\end{array}$ & $\begin{array}{l}\text { False-negative and positive rate } 11 \%(89 \% \\
\text { sensitivity and specificity) } \\
\text { Detect } 91 \% \text { of non-palpable cancers } \\
\text { Remark: Predicts the risk factor for future } \\
\text { development of breast cancer more accurately } \\
\text { than clinical or radiographic suspicion of } \\
\text { malignancy. }\end{array}$ & [33] \\
\hline 37,506 women & $\begin{array}{l}\text { Mobile } \\
\text { thermogram on } \\
\text { rural women }\end{array}$ & $\begin{array}{l}5.7 \text { cancers per } 1,000 \text { women } \\
12 \% \text { false-negative } \\
14 \% \text { false-positive } \\
\text { Remark: Early indication for } 10 \% \text { would have } \\
\text { a breast cancer. }\end{array}$ & [34] \\
\hline 4,393 women & $\begin{array}{l}\text { Concomitant } \\
\text { thermogram and } \\
\text { mammogram on } \\
\text { asymptomatic } \\
\text { patients }\end{array}$ & $\begin{array}{l}\text { Mammography found } 7 \text { cancers per } 1000 \text {. } \\
\text { IR found 1,028 patients with abnormal images } \\
\text { Combined methods } 24.1 \text { cancers per } 1000 \\
\text { Combined methods increase sensitivity by } 10 \% \\
\text { Remark: Mammography, infrared imaging and } \\
\text { other breast imaging techniques merely indicate } \\
\text { the presence of an abnormality. }\end{array}$ & {$[35,15]$} \\
\hline $\begin{array}{l}39,802 \text { women } \\
\text { (3 year period) }\end{array}$ & $\begin{array}{l}\text { Thermography, } \\
\text { physical } \\
\text { examination and } \\
\text { mammography }\end{array}$ & $\begin{array}{l}85 \% \text { sensitivity } \\
70 \% \text { specificity for thermography. } \\
\text { Remark: Thermography to shorten the list and } \\
\text { to detect earlier stage tumors. }\end{array}$ & [36] \\
\hline $\begin{array}{l}85,000 \text { patients } \\
10,834 \text { patients } \\
\text { (cold-stress) }\end{array}$ & $\begin{array}{l}\text { Therogram and } \\
\text { dynamic } \\
\text { thermogram }\end{array}$ & $\begin{array}{l}90 \% \text { sensitivity } \\
88 \% \text { specificity. } \\
\text { Remark: Cold stress decrease the false- } \\
\text { positive rate to } 3.5 \% \text { ( } 96.5 \% \text { sensitivity) }\end{array}$ & [37] \\
\hline $\begin{array}{l}4,716 \text { patients } \\
\text { (confirmed } \\
\text { carcinoma) } \\
3,305 \text { patients } \\
\text { (benign breast) }\end{array}$ & $\begin{array}{l}\text { Comparative } \\
\text { study among } \\
\text { clinical examination, } \\
\text { mammography, } \\
\text { and thermography }\end{array}$ & $\begin{array}{l}\text { Clinical examination: Sensitivity } 75 \% \text { overall } \\
\text { and 50\% in cancers less than } 2 \mathrm{~cm} \text { in size. } \\
\text { Mammography: Sensitivity } 80 \% \text { and specificity } \\
73 \% \text {. }\end{array}$ & [38] \\
\hline
\end{tabular}




\begin{tabular}{|c|c|c|c|}
\hline $\begin{array}{l}8,757 \text { general } \\
\text { women }\end{array}$ & $\begin{array}{l}\text { in the diagnosis of } \\
\text { breast cancer }\end{array}$ & $\begin{array}{l}\text { Thermography: Sensitivity } 88 \%(85 \% \text { in } \\
\text { tumors less than } 1 \mathrm{~cm} \text { in size) and specificity } \\
85 \% \text {. } \\
\text { Remarks: None of the techniques available is } \\
\text { stand alone. } \\
\text { Multimodal approach can give the best result. }\end{array}$ & \\
\hline 4,000 patients & $\begin{array}{l}\text { Mammography } \\
\text { and thermography } \\
\text { used separately } \\
\text { and together. }\end{array}$ & $\begin{array}{l}130 \text { sub-clinical carcinomas ranging in } \\
\text { diameter of } 3-5 \mathrm{~mm} \text {. } \\
10 \% \text { were detected by mammography only, } \\
50 \% \text { by thermography alone, } \\
40 \% \text { by both techniques. }\end{array}$ & [39] \\
\hline $\begin{array}{l}106 \text { patients } \\
\text { (negative) } \\
372 \text { patients } \\
\text { (positive) } \\
\text { physical and/or } \\
\text { mammogram }\end{array}$ & $\begin{array}{l}\text { Survival benefit } \\
\text { study } \\
\text { (subjected } \\
\text { identical } \\
\text { treatment } \\
\text { followed for } 5 \\
\text { years) }\end{array}$ & $\begin{array}{l}\text { Survival rate increased by } 61 \% \text { if the patients } \\
\text { medicated with initial thermographic } \\
\text { abnormalities. } \\
\text { Remark: Early identification of women at high } \\
\text { risk of breast cancer based on the objective } \\
\text { thermal assessment of breast health results in a } \\
\text { dramatic survival benefit. }\end{array}$ & [40] \\
\hline 58,000 women & $\begin{array}{l}\text { thermography } \\
\text { Cancer risk } \\
\text { assessment }\end{array}$ & $\begin{array}{l}40 \% \text { of } 1,527 \text { patients with initially healthy } \\
\text { breasts but abnormal thermograms developed } \\
\text { malignancies within } 5 \text { years. } \\
\text { Remark: Abnormal thermogram is the single } \\
\text { most important marker of high risk for the } \\
\text { future development of breast cancer. }\end{array}$ & [41] \\
\hline $\begin{array}{l}1,416 \text { patients } \\
165 \text { patients } \\
\text { (non-palpable } \\
\text { cancers) }\end{array}$ & $\begin{array}{l}\text { Isolated abnormal } \\
\text { breast } \\
\text { thermograms }\end{array}$ & $\begin{array}{l}\text { Isolated abnormal thermogram is associated } \\
\text { with an actuarial breast cancer risk of } 26 \% \text { at } 5 \\
\text { years. } \\
\text { In non-palpable the risk is } 53 \% \text { at initial } \\
\text { evaluation. } \\
\text { Remarks: } \\
\text { 1) A persistently abnormal thermogram, } \\
\text { even in the absence of any other sign of } \\
\text { malignancy, is associated with a high risk of } \\
\text { developing cancer, } \\
\text { 2) This isolated abnormal carries a high } \\
\text { risk of developing interval cancer, } \\
\text { 3) Abnormal thermograms is the first } \\
\text { warning sign for breast cancer }\end{array}$ & [42] \\
\hline
\end{tabular}

Studies showed that thermogram's first-line breast cancer detection strategy depends essentially on clinical examination and mammography. Though, mammography is accepted as the most reliable and cost-effective imaging modality, its contribution continues to be challenged with persistent false-negative rates ranging up to 30\% [43]; with decreasing sensitivity in patients on 
estrogen replacement therapy [44]. In addition, there is recent data suggesting that denser and less informative mammography images are precisely those associated with an increased cancer risk [45]. Echoing some of the shortcomings of the BCDDP concerning their study design and infrared imaging, Moskowitz indicated that mammography is also not a procedure to be performed by the untutored [46].

With the current emphasis on earlier detection, there is now renewed interest in the parallel development of complimentary imaging techniques that can also exploit the precocious metabolic, immunological and vascular changes associated with early tumor growth. While promising, techniques such as scintimammography [45], Doppler ultrasound [44], and MRI [47], are associated with a number of disadvantages that include exam duration, limited accessibility, need of intravenous access, patient discomfort, restricted imaging area, difficult interpretation and limited availability of the technology. Like ultrasound, they are more suited to use as second-line options to pursue the already abnormal clinical or mammographic evaluation. While practical, this step-wise approach currently results in the non-recognition, and thus delayed utilization of secondline technology in approximately $10 \%$ of established breast cancers. This is consistent with study published by Keyserlingk et al [48].

The above discussion proved that thermography had already paved its way as a promising adjunct screening tool. However, the sensitivity of this equipment varies with tumor size and the incidence of false positive result is very high. Moreover, medical application of infrared thermography was limited in the past years mainly due to the complexity, high cost and poor sensitivity provided by the output data generated from infrared cameras that were available at that time. Nowadays, advances in the infrared technology have again promoted its medical application as a promising non-invasive tool for imaging the functionality of superficial layers of tissues and the influence of vascular, neurogenic and metabolic processes that affect them. A high accuracy data acquisition system is available, but a big challenge is in deriving a solid correlation between superficial surface temperature and physiological bio-thermal parameters of a tumor, which has yet to be resolved. Numerous efforts have utilized the modern IR technology to predict the location, size and thermal parameters of tumors from the abnormal temperature profiles during the last few decades which included anatomical modeling or analogous modeling and inverse solution approach. 
Because of thermography's unique ability to image the thermovascular aspects of the breast, extremely early warning signals (from 8-10 years before any other detection method) have been observed in long-term studies. Consequently, thermography is the earliest known indicator for the future development of breast cancer. It is for this reason that an abnormal infrared image is the single most important marker of high risk for developing breast cancer. Thus, thermography has a significant place as one of the major front-line methods of breast cancer detection.

Studies performed before the 2000s have proved that thermogram is a promising screening tool to determine early stage benign tumor accurately much sooner before mammograms. Recent research is explaining the reason behind the abnormal thermal pattern over the skin under which hyperactive cells have grown. In general the body surface temperature is controlled by the local metabolism, blood circulation underneath the skin, and the heat exchange between the skin and its environment $[14,49]$. Change in any of these parameters could influence temperature distribution and heat flux at skin surface and thus reflect the physiological state of the human body. In particular the inflammation, metabolic rate, interstitial hypertension, abnormal vessel morphology and lack of response to homeostatic signals are some of the particular features that make tumors behave differently than normal tissues in terms of heat production and dissipation [14]. Therefore, an abnormal skin temperature profile can be used to predict the location, size and thermal parameters of the hyperactive region as well as follow up the treatment procedure. A number of previous papers show that the tissues in a hyperactive nodule exhibit remarkably higher metabolic and blood perfusion rate and slightly higher thermal conductivity. Gautherie et al. [37]found the following hyperbolic relationship between the metabolic heat generation and the tumor doubling time after experimenting on 84 patients with relatively small cancer (tumor size between 9 and $38 \mathrm{~mm}$ ). In another paper Gautherie [31] showed that the increased blood perfusion caused an enhancement in thermal conductivity. His in situ mode experimental findings were also supported by Molls et al. [50] who observed that an increase in blood flow of $150 \mathrm{ml} / 100 \mathrm{~g} / \mathrm{min}$ causes an increase in thermal conductivity of $0.05[\mathrm{~W} /(\mathrm{m} . \mathrm{K})]$.

In order to establish the correlation the internal temperature distribution in tissues was obtained by solving steady sate Pennes' bio-heat equation analytically with different boundary conditions [51,52] and the influence of thermal conductivity, metabolism, blood perfusion and heat exchange rate was studied for homogeneous and isotropic tissues [52]. Considering the geometrical and behavioral variation of biological tissues, which are common phenomenon for the 
case of tumor or cancer, numerical methods such as the finite difference method (FDM) [53], the finite element method (FEM) [14], and the boundary element method (BEM) [54] have been developed. All of the above mentioned research showed the correlation between bio-thermal parameter and temperature profile.

However, medical application of infrared thermography, that invokes indirect solution, was limited in the past years mainly due to the complexity, high cost and poor sensitivity provided by the output data generated from infrared cameras that were available at that time. Nowadays, advances in the infrared technology have again promoted its medical application as a promising non-invasive tool for imaging the functionality of superficial layers of tissues and the influence of vascular, neurogenic and metabolic processes that affect them. Lawson [12] was the first to propose the use of thermography detection of breast cancer, when he observed that the local temperatures of the skin over a tumor were significantly higher (approximately $2^{\circ} \mathrm{C}-3^{\circ} \mathrm{C}$ ) than the normal skin temperatures. Lawson and Chughtai [13] established that the region temperature difference over an embedded tumor was due to convection effects associated with increased blood perfusion, and the increased metabolism around the tumor. Further aspects of IR imaging techniques and detection methods of breast cancer from infrared images are described in detail by Diakides and Bronzino [6]. In [19] Santa Cruz et al. after making a comparative investigation between thermography and boron neutron capture therapy (BNCT), concluded that the thermography, a potential imager of tissue functionalities, can help to locate abnormally high temperature regions as well as melanoma nodules that are virtually invisible in CT images. Therefore, the analyses proved that the IR imaging can be applied as an important tool in the tumor screening process.

Research, mostly carried out in the last decade, observed the theoretical studies of the thermal behavior of the human organ by Direct Solving the Pennes bio-heat transfer equation analytically. Tissue interior thermal features had been anticipated for the overly simplistic rectangular and tubular homogeneous tissues models in [52, 51] and [55, 56], respectively. However, actual human organ tissues exhibit complex nonlinear, anisotropic, non-homogeneous, time, and rate-dependent behavior. In addition, the approach cannot provide the $2 \mathrm{D}$ temperature distribution over the skin surface. Consequently, bio-thermal parameters derived analytically are trivial and meaningless in clinical application. Several contemporary researchers incorporated thermal-electric analogy to find the parameters of a hot nodule $[21,57]$ by mapping the temperature 
texture over skin of a homogeneous tissue equivalent adiposity phantom. But, due to the interdependency of parameters, unknown shape and thermal behavior of tumor, the solution is murky and cannot be applied for clinical purposes readily. Numerous numerical approaches [58, 59] had been addressed on realistic (anatomical accurate) models to reveal the thermal feature of the female breast. The bio-heat problem was solved in the 3D rectangular tissue model [14] and breast model [60] to forecast the superficial temperature texture over a flat body organ and a female breast, respectively. These numerical methods, performed in the latter part of last decade, showed the correlation between bio-thermal parameters and the skin surface temperature profiles.

Moreover, several recent investigations utilized the correlation between the skin temperature and physiological parameters. As a result the screening tool turned into a useful diagnostic tool. Mital et al. [60] developed an in-vitro mode experimental and evolutionary method, to determined parameters of an embedded heat source representing a tumor using IR. An approximate heat source model of an embedded tumor was developed to determine the correlation among depth, radius and heat generation with skin temperature profile [21, 57]. Angelli et al. [14] used Finite difference method (FDM) and pattern search algorithm for estimating the depth, size and heat generation of an embedded tumor.

Literature survey indicates that, only a few works $[14,60]$ have been conducted to estimate tumor parameters from a thermogram image. All the studies have numerous limitations. For example, the anatomically accurate physical model of an organ considering its geometry and the tissue heterogeneity is an important feature to achieve accurate results. In addition, structural deformations merely due to body posture or some other disorder should be taken into account for developing the physical model. The adipose mode developed in [16] and the rectangular model in [14] is overly simplistic and premature. Though the proposed breast model in [25] is anatomically accurate, the assumptions that were made to design tissue layers for all the models were inappropriate and inaccurate. In conclusion, results obtained from those studies were not validated and were not suitable for clinical application. In addition, the Inverse Approach based on the Artificial Neural Network (ANN) [60, 57], the Genetic Algorithm (GA) [16], the pattern search algorithms [14] etc. to estimate the physical and thermal parameters of a tumor were not validated with real/clinical data. 
The objective of this study is the development of a novel methodology for correlating surface temperatures with hyperactive cell's thermal and physical behavior. The interior temperature distribution in tissues is obtained by solving the Pennes' bioheat equation analytically at a steady state with different boundary conditions and the influence of thermal conductivity, metabolism, blood perfusion and heat exchange rate studied for homogeneous and isotropic tissues. Though, the solutions are not useful for clinical application as long as they couldn't be applied to nonhomogeneous and anisotropic tissues, and also they couldn't be applied to anticipate the skin temperature distribution but they are applied to validate the numerical results obtained in the second stage. Anatomical accurate models of human superficial organs are developed in consideration of the geometrical and behavioral variation of biological tissues which is obvious for tumor or cancer, and the problem has been addressed in numerical methods.

Most of the previous works restricted the diagnosis process for breast cancer patients only. However, the idea of this work is to use thermography- temperature profiles to predict the location, size and metabolism of tumors embedded in any outer body organs of humans. In general, the superficial organs of the human body have flat, tubular and curved outlook. Therefore, the organs are modeled either as a solid rectangle, cylinder or hemisphere respecting to their physical structure and the tumor is modeled as a spherical heat source. Tumor's bio-thermal and physical features and the host organ's structure viz. the presence of local blood vessels, constituent tissues, heat loss surface area etc. may affect the temperature distribution. To investigate the anatomical effect, the bio-heat transfer model with non-linear boundary conditions was developed for the above mentioned body geometries, using finite element analysis software.

Continuing with the ongoing research for breast tumors, this study has also taken into account breast deformation caused by gravity. It has been noted, however, that the breast geometry may deform substantially merely because of body posture changes [61], and that the nonlinear elasticity effects associated with large deformations may not be negligible [62]. The study develops physical models for ptosis breast. Ptosis is a phenomenon of breast sagging due to aging, malnutrition, skin elasticity, etc. The forecasting of ptosis breast tumor parameters is an innovative thought of this study.

Then an inverse approach is proposed, applying an optimization method to estimate thermal and physical parameters of a tumor, from which surface temperature data may be obtained from 
clinic or infrared thermogram or finite element model. The study has applied both the Genetic Algorithm (GA) and Artificial Neural Network (ANN) optimization approaches to forecast the thermal and physiological parameters of tumors developed in the forearm, breast and chest. The forecasted data also underwent another optimization process, the Pattern Search Algorithm, to ensure the highest precision. The "Handbook of Genetic Algorithm" [63] covers a wide range of application of GA's in the real world and it can be seen that GA can be used as robust non-gradient based optimization procedures. ANNs are one of the burgeoning areas of current research and attract people from a wide variety of disciplines of science and technology. The important features and applications of ANN are discussed in [64]. The pattern search algorithm is a powerful approach for bound constraint minimization and discussed detail in $[65,66]$.

\subsection{Summary}

The large patient populations and long survey periods in many of the above clinical studies yield a high significance to the various statistical data obtained. This is especially true for the contributions of thermography to early cancer diagnosis, as an invaluable marker of high-risk populations, and therapeutic decision making (a contribution that has been established and justified by the unequivocal relationship between heat production and tumor doubling time).

Currently available high-resolution digital infrared imaging (Thermography) technology benefits greatly from enhanced image production, standardized image interpretation protocols, computerized comparison and storage, and sophisticated image enhancement and analysis. Over 30 years of research and 800 peer-reviewed studies encompassing well over 300,000 women participants has demonstrated thermography's abilities in the early detection of breast cancer. Ongoing research into the thermal characteristics of breast pathologies will continue to investigate the relationships between neoangiogenesis, chemical mediators, and the neoplastic process. Moreover, an indirect solution using evolutionary method is still in the way to apply in thermogram diagnostics.

It is unfortunate, but many physicians still hesitate to consider thermography as a useful tool in clinical practice in spite of the considerable research database, continued improvements in both thermographic technology and image analysis, and continued efforts on the part of the thermographic societies. This attitude may be due to the fact that the physical and biological bases of thermography are not familiar to most physicians. The other methods of cancer investigations 
refer directly to topics of medical teaching. For instance, radiography and ultrasonography refer to anatomy. Thermography, however, is based on thermodynamics and thermos-kinetics, which are unfamiliar to most physicians, though man is experiencing heat production and exchange in every situation he undergoes or creates.

Considering the contribution that thermography has demonstrated thus far in the field of early cancer detection, all possibilities should be considered for promoting further technical, biological, and clinical research in this procedure. 


\section{Chapter 3 Thermal Analysis in Healthy Homogeneous Tissues}

\subsection{Introduction}

Solutions of bio-heat transfer problems on the developed models have been discussed in this chapter and the obtained solutions have been applied for finding interior and exterior temperature distribution of the above-mentioned organs. Heat and temperature flow in a living biological body was first modelled by Pennes in 1948 [67]. The general theory about temperature distribution in tissue interior was obtained by analytic solving the Pennes' bio-heat transfer model, basically a second order differential equation, with known boundary conditions. Tissues interior dynamic thermal feature substantially recommended for hyperthermia (also called thermal therapy or thermotherapy) treatment (cancer killing by exposing tissue with high temperature). However, thermal imaging (thermogram), recently proved as a gold standard for breast cancer screening, requires the Penne's steady-state thermal model. The time-invariant bio-thermal model with known boundaries is applied to compute the temperature flow inside tissues which will be the main focus in this chapter. Tissues have distinctive thermal features because of their unique exterior (over which heat exchange occurs) and heat flow direction (heat gushing prominent in particular direction). Mathematical formulations involved in solving the thermal problems analytically with a coordinate system which can express the tissue geometry (in which the temperature distribution will be measured) conveniently. For example, the Cartesian system is the best choice for cuboid tissue, the Cylindrical system is for tubular tissues and the Spherical system is for curvy tissues. Therefore, an organ's interior thermal character will be estimated by solving the heat equation fitting the organ on resembling coordinate systems.

The objective of this chapter is developing a homogenous tissue model of external organs in the human body mimicking the physical exterior and solving the second order heat transfer equation with non-linear boundary conditions and finally estimating the radial temperature flow. Analytical approach for spatial temperature distribution is detailed thoroughly in this chapter. The major drawback of the analytical approach is that they can't apply to inhomogeneous models handily. Rather, they require numerical approaches for solving multi-tissue organ's thermal problems. However, the analytical approaches still stand as a powerful validating tool for numeric 
results. The comparison between analytical and numerical results is presented at a later part of this chapter.

During the last 60 years, several efforts had been carried out in solving the bio-heat model (a landmark model) proposed by Pennes. Wissler solved the Pennes model at steady-state and dynamic conditions with cylindrical systems in 1998 and remarked that the proposed (by Pennes) model is pertinent for estimating thermal behavior of biological tissues [68]. The model was, at first, applied for estimating the radial heat flow in cylindrical tissues which had been set up for application in limb and whole body heat transfer studies using Bessel's equation and the corresponding analytical solution was derived in $[55,56]$. The author revealed the effect of thermal conductivity, blood perfusion, metabolic heat generation and heat losses on the temperature distribution. Zhou et al. [51] applied the Cartesian system to expose the interior heat transfer in a single direction with asserting several boundary conditions and discussed how the temperature changes with tissue thickness. Cartesian geometry was also applied in solving the temperature distribution in skin tissues with four different heat exchange conditions i.e. the Dirichlet, Neumann, Convective and Radiation conditions.

Research has also been conducted to obtain an analytical solution on Pennes' dynamic bioheat model with different boundary conditions. Tzu-Ching Shih et al. [69] studied the effect of the temperature response of a semi-infinite biological tissue due to a sinusoidal heat flux at skin. A similar analysis had also been conducted in [70] with generalized spatial and transient heating on skin and inside the biological bodies using Green's function. The dynamic analysis results may be useful for therapeutic treatment, thermal comfort analysis, tissue parameter estimation, thermal injury estimation and hyperthermia tumor ablution.

This chapter covers a step-by-step process in solving the steady-state bio-heat equation analytically with perfect heat exchange boundary (section 4.2), the estimated temperature profiles in tissue inside (section 4.3) and will also show the merit of applying matched coordinate (section 4.4). Across-check between the analytical estimate and numerical result (section 4.3) will be articulated. With revealing the attributive physio-thermo-biological parameters, the empirical effect of each parameter on the temperature profile will be examined and cross-checked. 


\subsection{Bio-heat Transfer Model}

Pennes' developed a bio-heat model considering blood perfusion and artier blood temperature effect on in-tissue temperature distribution which is presented as:

$$
\rho c \frac{d T}{d t}=k \nabla^{2} T+\omega_{b} c_{b}\left(T_{a}-T\right)+Q_{m}
$$

where $\rho$ and $c$ refer to the density and specific heat of tissue, respectively, $\omega_{b}$ is the product of blood density and perfusion rate per unit volume of tissue, $c_{b}$ is the specific heat of blood, $T_{a}$ is the artier blood temperature and $Q_{m}$ is the rate of metabolic heat generation per unit volume of tissue.

In particular tissue, the temperature flow is controlled by the blood circulation rate, metabolism and thermal conductivity. Changes in any of these parameters, most likely because of tumor, can induce variation in flow rate and spatial distribution of temperature and heat flux at skin surface. However, the dynamic temperature flow rate becomes negligible for the thermal quantities, such as the metabolic rate, perfusion rate, and the heat exchange rate remains unchanged over time as well and for the slow growing tumors. Therefore, the steady-state bio-heat equation is useful for thermal analysis of living organs, which can be written as

$$
k \nabla^{2} T+\omega_{b} c_{b}\left(T_{a}-T\right)+Q_{m}=0
$$

Eqn. (4.2) can be applied to solve the physical models proposed in Chapter 34. Reviewing the proposed realistic models for the human organs, shown in Figure 4-6, 4-8 and 4-10, indicated several layers (domain) where each domain has different thermo-physical character. Eqn. (3.2) can be modified for multi-domain model as:

$$
k_{i} \nabla^{2} T+\omega_{b i} c_{b i}\left(T_{a}-T\right)+Q_{m i}=0
$$

where the suffix $i=1,2,3$, 4 , each number stands for a domain.

In addition the Laplacian operator $\left(\nabla^{2}\right)$ in Eqn. (4.2) can be written as:

$$
\begin{gathered}
\nabla^{2}=\left(\frac{\partial}{\partial x}\right)^{2}+\left(\frac{\partial}{\partial y}\right)^{2}+\left(\frac{\partial}{\partial z}\right)^{2}, \text { in the Cartesian system } \\
\nabla^{2}=\frac{1}{\rho} \frac{\partial}{\partial \rho}\left(\rho \frac{\partial}{\partial \rho}\right)+\frac{1}{\rho^{2}}\left(\frac{\partial}{\partial \varphi}\right)^{2}+\left(\frac{\partial}{\partial z}\right)^{2}, \text { in the Cylindrical system } \\
\nabla^{2}=\frac{1}{r^{2}} \frac{\partial}{\partial r}\left(r^{2} \frac{\partial}{\partial r}\right)+\frac{1}{r^{2} \sin \theta} \frac{\partial}{\partial \theta}\left(\sin \theta \frac{\partial}{\partial \theta}\right)+\frac{1}{r^{2} \sin ^{2} \theta}\left(\frac{\partial}{\partial \varphi}\right)^{2}, \text { in the Spherical system }
\end{gathered}
$$


With selecting the coordinate system and the boundaries, heat flow in any direction can be estimated. However, the exterior of the organ's model dictates the suitable coordinate system and the symmetry of the model indicates the prominent heat flow direction(s), and consequently, the boundary conditions. The fundamental three different models (except the droopy breast models) are the cuboid (rectangle box) or the cylinder or the hemisphere shapes. In case of a cuboid model $(x \ll y(=z))$ if placed the center of $x=0$ plane at origin, the prominent heat flow will happen in $x$ direction. Similarly for the origin-geometric center cylindrical model $(\rho \ll z)$ the thermal flux will be directed in $\rho$ axis. Likewise, if the base plane is thermally insulated the heat will be guided toward radial $(r)$ direction for hemispherical model. Therefore, Eqn. (4.2) can be rewritten for different geometry as:

$$
\begin{gathered}
k_{i}\left(\frac{\partial}{\partial x}\right)^{2} T+\omega_{b i} c_{b i}\left(T_{a}-T\right)+Q_{m i}=0 \quad(\text { Cartesian }) \\
k_{i} \frac{1}{\rho} \frac{\partial}{\partial \rho}\left(\rho \frac{\partial T}{\partial \rho}\right)+\omega_{b i} c_{b i}\left(T_{a}-T\right)+Q_{m i}=0 \quad \text { (Cylindrical) } \\
k_{i} \frac{1}{r^{2}} \frac{\partial}{\partial r}\left(r^{2} \frac{\partial T}{\partial r}\right)+\omega_{b i} c_{b i}\left(T_{a}-T\right)+Q_{m i}=0 \quad \text { (Spherical) }
\end{gathered}
$$

where $i$ indicates the layers of tissues in an organ where the layers have completely different bio-thermal features from one another and come up with $i$-number of heat transfer equations.Solving the multiple thermal equations for such a multi-layer (heterogeneous) structure analytically is impossible. However, to comprehend how an organ's shape influences the tissue interior thermal profiles a simplified homogeneous model will suffice. Another useful application of receiving the basic thermal features on the approximate models, as mentioned earlier, is to confirm the numerical accuracy.

\subsection{Thermal Analysis of Tissues}

The thermal analyses on the cuboid, cylindrical and (hemi) spherical homogeneous models are discussed in the following section. 


\subsubsection{Cuboid (Rectangular box) tissues}

The cuboid model is shown in Figure 4-22, the dimension ' $L$ ' is the model (tissue) thickness i.e. depth between skin and body core, ' $\mathrm{W}$ ' is the model width and ' $\mathrm{H}$ ' is the model height [W (or

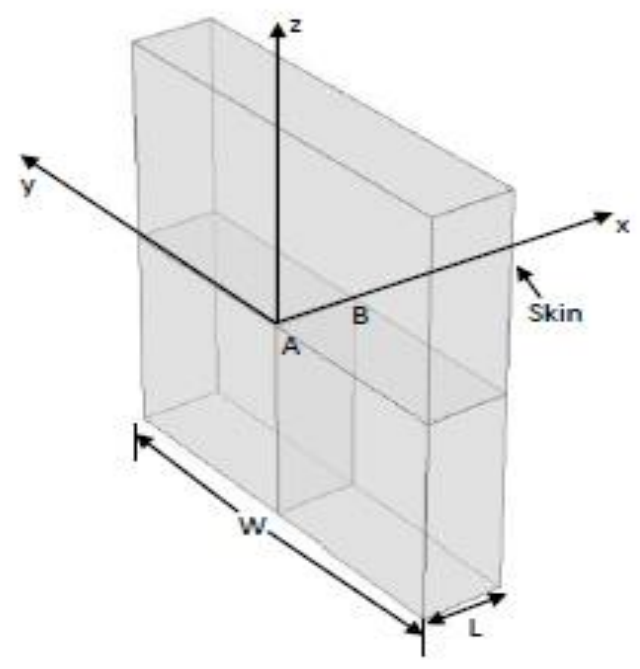

Figure 4-1 Homogeneous cuboid tissue model

H) $>>\mathrm{L}$ ]; ' $\mathrm{A}$ ' is the model center. Obviously, the model has symmetry in y and z-axes, therefore, the lateral boundaries are assumed to be thermally insulated i.e. no heat flow in these directions.

Assuming the core plane hold at the constant artier temper and the model surface is perfect heat exchanger i.e. emanated heat fluxes entirely exchange with environment. Therefore, the onedimensional steady-state Pennes' bio-heat equation for homogenous cuboid model can be written as: 


$$
k \frac{d^{2} T}{d x^{2}}+\omega_{b} c_{b}\left(T_{a}-T\right)+Q_{m}=0
$$

Eqn. (3.3) can be rewritten as:

Table 3-1 Boundary Conditions

\begin{tabular}{|c|c|c|}
\hline Boundary Set & Conditions & Remarks \\
\hline BC-I & $\begin{array}{ll}x=0, & T=T_{c} \\
x=d, & T=T_{s}\end{array}$ & $\begin{array}{l}\text { Core temperature }\left(T_{c}\right) \text { and surface } \\
\text { temperature }\left(T_{S}\right) \text { known }\end{array}$ \\
\hline BC-II & $\begin{array}{ll}x=0, & \frac{d T}{d x}=0 \\
x=d, & T=T_{s}\end{array}$ & $\begin{array}{l}\text { Thermal insulated core, } \\
\text { Surface temperature }\left(T_{S}\right) \text { known }\end{array}$ \\
\hline BC-III & $\begin{array}{c}x=0, T=T_{c} \\
x=d,-k \frac{d T}{d x}=h_{a}\left(T-T_{e}\right)\end{array}$ & $\begin{array}{l}\text { Core temperature }\left(T_{c}\right) \text { known, } \\
\text { Surface purely emissive }\end{array}$ \\
\hline BC-IV & $\begin{array}{c}x=0, \quad \frac{d T}{d x}=0 \\
x=d,-k \frac{d T}{d x}=h_{a}\left(T-T_{e}\right)\end{array}$ & $\begin{array}{l}\text { Thermal insulated core, } \\
\text { Surface purely emissive }\end{array}$ \\
\hline
\end{tabular}

where $\alpha=\frac{\omega_{b} c_{b}}{k}$ and $\beta=\frac{\omega_{b} c_{b} T_{a}+Q_{m}}{k}$. The general solution of Eqn.(3.4) is obtained as:

$$
T(x)=C_{1} e^{-\alpha x}+C_{2} e^{\alpha x}+\frac{\beta}{\alpha}
$$

The evaluation of arbitrary constants $C_{1}$ and $C_{2}$ is determined using boundary conditions. Some possible sets of boundary conditions are presented in Table 3.1. The BC-I (Boundary Condition-I) indicates both the tissue core and skin maintain constant temperatures - artier blood temperature $\left(T_{c}\right)$ and skin temperature $\left(T_{S}\right)$, respectively, known as Dirichlet condition; whereas in BC-II, the core flows the Fourier heat flow law; while a complete heat exchange at skin surface is assumed in BC-III. Unlike above conditions which have at least one side fixed, the BC-IV is more flexible and invariable to ambient or system.

Applying BC-I in Eqn. (3.5),

$$
C_{1}+C_{2}=T_{c}-\frac{\beta}{\alpha}
$$




$$
C_{1} e^{-\sqrt{\alpha} d}+C_{2} e^{\sqrt{\alpha} d}=T_{s}-\frac{\beta}{\alpha}
$$

Solving for $C_{1}$ and $C_{2}$ in Eqns. (3.6),

$$
C_{1}=\frac{T_{s}-\frac{\beta}{\alpha}-e^{\sqrt{\alpha} d}\left(T_{c}-\frac{\beta}{\alpha}\right)}{e^{\sqrt{\alpha} d}-e^{-\sqrt{\alpha}}} \text { and } C_{2}=\frac{e^{-\sqrt{\alpha} d}\left(T_{c}-\frac{\beta}{\alpha}\right)-T_{s}+\frac{\beta}{\alpha}}{e^{\sqrt{\alpha} d}-e^{-\sqrt{\alpha} d}}
$$

Substituting the values in Eqn. (3.5),

$$
\begin{gathered}
T(x)=\frac{T_{s}-\frac{\beta}{\alpha}-e^{\sqrt{\alpha} d}\left(T_{c}-\frac{\beta}{\alpha}\right)}{e^{\sqrt{\alpha} d}-e^{-\sqrt{\alpha} d}} e^{-\alpha x}+\frac{e^{-\sqrt{\alpha} d}\left(T_{c}-\frac{\beta}{\alpha}\right)-T_{s}+\frac{\beta}{\alpha}}{e^{\sqrt{\alpha} d}-e^{-\sqrt{\alpha} d}} e^{\alpha x}+\frac{\beta}{\alpha} \\
T(x)=\frac{\left(T_{s}-\frac{\beta}{\alpha}\right)\left(e^{-\sqrt{\alpha} x}-e^{\sqrt{\alpha} x}\right)+\left(T_{c}-\frac{\beta}{\alpha}\right)\left(e^{-\sqrt{\alpha}(d-x)}-e^{\sqrt{\alpha}(d-x)}\right)}{e^{\sqrt{\alpha} d}-e^{-\sqrt{\alpha} d}}+\frac{\beta}{\alpha} \\
T(x)=\frac{\left(T_{s}-\frac{\beta}{\alpha}\right) \sinh (\sqrt{\alpha} x)+\left(T_{c}-\frac{\beta}{\alpha}\right) \sinh (\sqrt{\alpha}(d-x))}{\sinh (\sqrt{\alpha} d)}+\frac{\beta}{\alpha}
\end{gathered}
$$

Similarly BC-II, III and IV give, respectively,

$$
\begin{gathered}
T(x)=\frac{\left(T_{s}-\frac{\beta}{\alpha}\right) \cosh (\sqrt{\alpha} x)}{\cosh (\sqrt{\alpha} d)}+\frac{\beta}{\alpha} \\
T(x)=\frac{\frac{h_{a}}{k}\left(T_{e}-\frac{\beta}{\alpha}\right) \sinh (\sqrt{\alpha} x)+\left(T_{c}-\frac{\beta}{\alpha}\right)\left[\sqrt{\alpha} \cosh (\sqrt{\alpha}(d-x))+\frac{h_{a}}{k} \sinh (\sqrt{\alpha}(d-x))\right]}{\sqrt{\alpha} \cosh (\sqrt{\alpha} d)+\frac{h_{a}}{k} \sinh (\sqrt{\alpha} d)}+\frac{\beta}{\alpha} \\
T(x)=\frac{\frac{h_{a}}{k}\left(T_{e}-\frac{\beta}{\alpha}\right) \cosh (\sqrt{\alpha} x)}{\sqrt{\alpha} \sinh (\sqrt{\alpha} d)+\frac{h_{a}}{k} \cosh (\sqrt{\alpha} d)}+\frac{\beta}{\alpha}
\end{gathered}
$$

Eqns. (3.9) through (3.12) present the temperature expressions in tissue interior of rectangular box homogeneous tissues under four different boundary conditions, respectively (see Table 4-3). Because of the rigidness of the Dirichlet conditions (BC-I), the expression in Eqn. (3.9) will be convenient for in vitro thermal estimation, for example, determining the thermal features of adipose in a fully controlled laboratory condition. The semi-rigid expressions in Eqns. (3.10) and (3.11) can also be applied to the in-vitro experiments. Unlike other solutions, the thermal expression in Eqn. (3.11) is flexible and reluctant to ambient, therefore, might be suitable for invivo (in situ) or clinical applications. 


\subsubsection{Cylindrical (Tubular) tissues}

Relating to the tubular model (see Figure 4.8), the approximate thermal plot inside the simplistic model as shown in Figure 4-23 centering on z-axis (point A) with axial and glide plane symmetry, will be estimated. Assuming the radius of the model $\rho$ is much smaller than the height, resulting prominent heat flowing toward the surface on $x-y$ plane. Therefore, the spatial temperature plot is a function of $\rho$ only, hence, the heat flow expression in Eqn. 3.2 has been rewritten for homogenous tubular organs as:

$$
\frac{1}{\rho} \frac{d}{d \rho}\left(\rho \frac{d T}{d \rho}\right)+\frac{\omega_{b} c_{b}}{k}\left(T_{a}-T\right)+\frac{Q_{m}}{k}=0
$$

Assuming, Eqn. (3.13) governs the thermal features of the simplistic model of a cylindrical organ (or segment), in particular, the portion of forearm which is closer to the elbow and considering the imaginary center axis $(z-$ axis) as the body core and attributing thermal insulation on it, while perfect heat loss on the outer surface, the boundary conditions become the following:

$$
\begin{aligned}
& \rho=0, \frac{d T}{d \rho}=0 \\
& \rho=d,-k \frac{d T}{d \rho}=h_{a}\left(T-T_{e}\right)
\end{aligned}
$$

Eqn. (3.13) and boundary conditions (3.14) are to be applied on the model shown in Fig. 3.2. Rationalizing Eqn. (3.13) and the boundary conditions (Eqns. 3.14) with the following characteristic quantities: 


$$
\rho^{*}=\frac{\rho}{d}, \quad T^{*}=\frac{T-T_{e}}{T_{a}-T_{e}}
$$

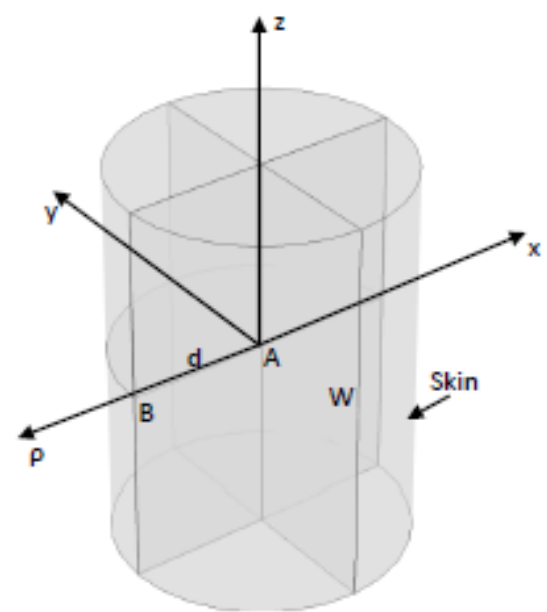

Figure 3-2 Homogeneous cylindrical tissue model

and substituting Eqn.(3.15) back into Eqn.(3.13):

$$
\frac{1}{\rho^{*}} \frac{d}{d \rho^{*}}\left(\rho^{*} \frac{d T^{*}}{d \rho^{*}}\right)+\frac{\omega_{b} c_{b} \rho^{2}}{k}\left(1-T^{*}\right)+\frac{Q_{m} \rho^{2}}{k\left(T_{a}-T_{e}\right)}=0
$$

the dimensionless parameters and variables are defined as follows:

$$
\omega_{b}^{*}=\frac{\omega_{b} c_{b} d^{2}}{k}, Q_{m}^{*}=\frac{Q_{m} d^{2}}{k\left(T_{a}-T_{e}\right)}, h_{a}^{*}=\frac{h_{a} d}{k}
$$

Hence, the original dimensional equation and the boundary conditions can be rewritten as:

$$
\frac{1}{\rho^{*}} \frac{d}{d \rho^{*}}\left(\rho^{*} \frac{d T^{*}}{d \rho^{*}}\right)-\omega_{b}^{*} T^{*}+\omega_{b}^{*}+Q_{m}^{*}=0
$$

and

$$
\left\{\begin{array}{c}
\rho^{*}=0, \frac{d T^{*}}{d \rho^{*}}=0 \\
\rho^{*}=1, \frac{d T^{*}}{d \rho^{*}}=-h_{a}^{*} T^{*}
\end{array}\right.
$$

Further, in order to standardize the equation, assume:

$$
A=\omega_{b}^{*}+Q_{m}^{*}, \quad B=\omega_{b}^{*}, \quad \varnothing=A-B T^{*}
$$

Thus substituting Eqn. (3.20) into Eqn. (3.18), we obtain 


$$
\frac{d^{2} \emptyset}{d \rho^{* 2}}+\frac{1}{\rho^{*}} \frac{d \emptyset}{d \rho^{*}}-B \emptyset=0
$$

Eqn. (3.21) is a zero order modified Bessel differential equation, whose general solution can be expressed as:

$$
R(z)=C_{1} I_{v}(z)+C_{2} K_{v}(z)
$$

where $I_{v}$ and $K_{v}$ are the modified Bessel functions of the second kind. In order to determine if the analytic solution can be expressed by Bessel functions, Eqn. (3.22) has been compared with the Generalized Bessel's equation as follows:

$$
\frac{d^{2} R}{d x^{2}}+\left[\frac{1-2 m}{x}-2 \alpha\right] \frac{d R}{d x}+\left[p^{2} a^{2} x^{2 p-2}+\alpha^{2}+\frac{\alpha(2 m-1)}{x}+\frac{m^{2}-p^{2} v^{2}}{x^{2}}\right] R=0
$$

The corresponding solution of Eqn. 3.23 is:

$$
R=x^{m} e^{\alpha x}\left[C_{1} J_{v}\left(a x^{p}\right)+C_{2} Y_{v}\left(a x^{p}\right)\right]
$$

where $J_{v}$ and $Y_{v}$ are the modified Bessel function of the first kinds respectively, $C_{1}$ and $C_{2}$ are arbitrary constants which can be obtained according to the given boundary conditions. The result of comparison between Eqn. (3.22) and (3.24) is shown below:

$$
\alpha=0, m=0, v=0, p=1, a^{2}=-B
$$

Hereby, the solution of Eqn. 3.20 can be expressed as:

$$
\emptyset=C_{1} I_{0}\left(\sqrt{B} \rho^{*}\right)+C_{2} K_{0}\left(\sqrt{B} \rho^{*}\right)
$$

Substituting 3.25 into 3.19 , the solution for $T^{*}$ can be written as:

$$
T^{*}=\frac{\omega_{b}^{*}+Q_{m}^{*}}{\omega_{b}^{*}}-\left[\frac{C_{1}}{\omega_{b}^{*}} I_{0}\left(\sqrt{\omega_{b}^{*}} \rho^{*}\right)+\frac{C_{2}}{\omega_{b}^{*}} K_{0}\left(\sqrt{\omega_{b}^{*}} \rho^{*}\right)\right]
$$

The next step is to determine the values of two arbitrary constants $C_{1}$ and $C_{2}$. According to the characteristics of Bessel's equation, when $z=0$, we have $I_{1}(0) \equiv 0$ and $K_{1}(0) \rightarrow \infty$. Considering the boundary conditions in Eqn. (3.18), some simple derivations lead to:

$$
C_{2} \equiv 0, \quad \frac{d T^{*}}{d \rho^{*}}=-\frac{\sqrt{\omega_{b}^{*}} C_{1}}{\omega_{b}^{*}} I_{1}\left(\sqrt{\omega_{b}^{*}} \rho^{*}\right)
$$


Putting $\rho^{*}=1, \frac{d T^{*}}{d \rho^{*}}=-h_{a}^{*} T^{*}$, we obtain:

$$
\begin{gathered}
-h_{a}^{*}\left(\frac{\omega_{b}^{*}+Q_{m}^{*}}{\omega_{b}^{*}}-\frac{C_{1}}{\omega_{b}^{*}} I_{0}\left(\sqrt{\omega_{b}^{*}}\right)\right)=-\frac{\sqrt{\omega_{b}^{*}} C_{1}}{\omega_{b}^{*}} I_{1}\left(\sqrt{\omega_{b}^{*}}\right) \\
C_{1}=\frac{h_{a}^{*}\left(\omega_{b}^{*}+Q_{m}^{*}\right)}{h_{a}^{*} I_{0}\left(\sqrt{\omega_{b}^{*}}\right)+\sqrt{\omega_{b}^{*}} I_{1}\left(\sqrt{\omega_{b}^{*}}\right)}
\end{gathered}
$$

So we have:

$$
\begin{gathered}
T^{*}\left(\rho^{*}\right)=\frac{\omega_{b}^{*}+Q_{m}^{*}}{\omega_{b}^{*}}-\frac{h_{a}^{*}\left(\omega_{b}^{*}+Q_{m}^{*}\right)}{\omega_{b}^{*}\left[h_{a}^{*} I_{0}\left(\sqrt{\omega_{b}^{*}}\right)+\sqrt{\omega_{b}^{*}} I_{1}\left(\sqrt{\omega_{b}^{*}}\right)\right]} I_{0}\left(\sqrt{\omega_{b}^{*}} \rho^{*}\right) \\
T^{*}\left(\rho^{*}\right)=\frac{\omega_{b}^{*}+Q_{m}^{*}}{\omega_{b}^{*}}\left[1-\frac{h_{a}^{*}}{\left[h_{a}^{*} I_{0}\left(\sqrt{\omega_{b}^{*}}\right)+\sqrt{\omega_{b}^{*}} I_{1}\left(\sqrt{\omega_{b}^{*}}\right)\right]} I_{0}\left(\sqrt{\omega_{b}^{*}} \rho^{*}\right)\right] \\
T^{*}\left(\rho^{*}\right)=\frac{\omega_{b}^{*}+Q_{m}^{*}}{\omega_{b}^{*}}\left[1-\frac{I_{0}\left(\sqrt{\omega_{b}^{*}} \rho^{*}\right)}{\left[I_{0}\left(\sqrt{\omega_{b}^{*}}\right)+\frac{\sqrt{\omega_{b}^{*}}}{h_{a}^{*}} I_{1}\left(\sqrt{\omega_{b}^{*}}\right)\right]}\right]
\end{gathered}
$$

Finally analytical solution for $T$ is given by:

$$
\begin{gathered}
\left.T=T_{e}+\left(T_{a}-T_{e}\right) \frac{\omega_{b}^{*}+Q_{m}^{*}}{\omega_{b}^{*}}\left[1-\frac{I_{0}\left(\sqrt{\omega_{b}^{*}} \rho^{*}\right)}{\left[I_{0}\left(\sqrt{\omega_{b}^{*}}\right)+\frac{\sqrt{\omega_{b}^{*}}}{h_{a}^{*}} I_{1}\left(\sqrt{\omega_{b}^{*}}\right)\right]}\right]\right] \\
\left.T=T_{e}+\left(T_{a}-T_{e}\right)\left[1+\frac{Q_{m}}{\omega_{b} c_{b}\left(T_{a}-T_{e}\right)}\right]\left[1-\frac{I_{0}\left(\sqrt{\frac{\omega_{b} c_{b}}{k}} \rho\right)}{\left[I_{0}\left(\sqrt{\frac{\omega_{b} c_{b} d^{2}}{k}}\right)+\frac{k \sqrt{\omega_{b}^{*}}}{h_{a} d} I_{1}\left(\sqrt{\frac{\omega_{b} c_{b} d^{2}}{k}}\right)\right]}\right]\right]
\end{gathered}
$$

In cylindrical organs temperature emanate towards the periphery and the radial flow is a function of the organ's radius $(\rho)$ only. 


\subsection{3 (Hemi)Spherical tissues}

The physical model of curved organ (see Figure 4.10) is approximated as a full-sphere filled with uniform tissue shown in Figure 4-24. At steady-state the temperature flow expression in Eqn. (3.2) is presented as in Eqn. (3.28) and the boundary conditions as in Eqn. (3.29):

$$
\frac{1}{r^{2}} \frac{d}{d r}\left(r^{2} \frac{d T}{d r}\right)+\frac{\omega_{b} c_{b}}{k}\left(T_{a}-T\right)+\frac{Q_{m}}{k}=0
$$

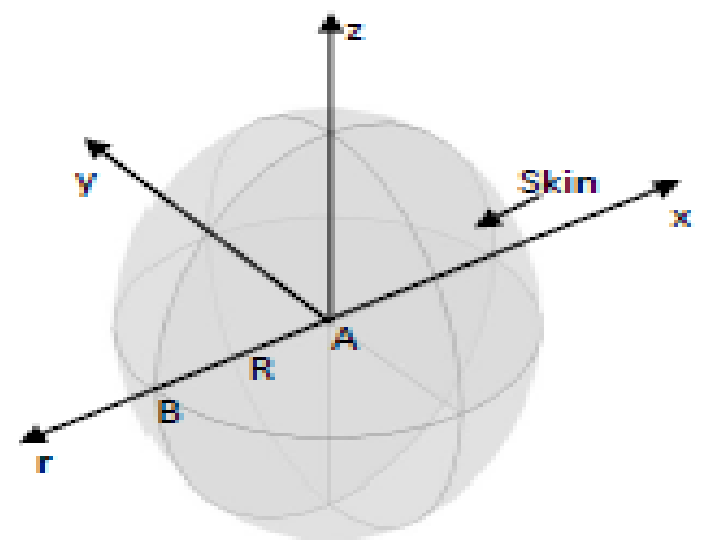

Figure 3-3 Homogeneous spherical tissue model

$$
r=0, \quad \frac{d T}{d r}=0 ; \quad r=d,-k \frac{d T}{d r}=h_{a}\left(T-T_{e}\right)
$$

Characteristics quantities to obtain the dimensionless heat equation and boundary conditions are as follows:

$$
r^{*}=\frac{r}{R}, \quad T^{*}=\frac{T-T_{e}}{T_{a}-T_{e}}
$$

Using Eqn. (4.30), Eqn. (4.28) can be written as:

$$
\frac{1}{r^{* 2}} \frac{d}{d r^{*}}\left(r^{* 2} \frac{d T^{*}}{d r^{*}}\right)+\frac{\omega_{b} c_{b} R^{2}}{k}\left(1-T^{*}\right)+\frac{q_{m} R^{2}}{k\left(T_{a}-T_{e}\right)}=0
$$

and the dimensionless boundary conditions are:

$$
r^{*}=0, \quad \frac{d T^{*}}{d r^{*}}=0
$$

and

$$
r^{*}=1, \quad \frac{d T^{*}}{d r^{*}}=-\frac{h_{a} R}{k} T^{*}
$$


The dimensionless parameters and variables are defined as:

$\frac{\omega_{b} c_{b} R^{2}}{k}=\omega^{*}, \frac{q_{m} R^{2}}{k\left(T_{a}-T_{e}\right)}=q_{m}^{*}$ and $\frac{h_{a} R}{k}=h_{a}^{*}$

Therefore, the dimensionless governing equation is:

$$
\frac{1}{r^{* 2}} \frac{d}{d r^{*}}\left(r^{* 2} \frac{d T^{*}}{d r^{*}}\right)-\omega_{b}^{*} T^{*}+\omega_{b}^{*}+q_{m}^{*}=0
$$

and the boundary conditions are:

$$
r^{*}=0, \quad \frac{d T^{*}}{d r^{*}}=0
$$

and

$$
r^{*}=1, \quad \frac{d T^{*}}{d r^{*}}=-h_{a}^{*} T^{*}
$$

By dropping the superscripts * and letting $\omega_{b}^{*}=\alpha$ and $\omega_{b}^{*}+q_{m}^{*}=\beta$, Eqn. (3.33) becomes:

$$
\frac{1}{r^{2}} \frac{d}{d r}\left(r^{2} \frac{d T}{d r}\right)-\alpha T+\beta=0
$$

To solve Eqn. (3.35), let's assume $T=\frac{H_{(r)}}{\sqrt{r}}$ and by differentiating gives,

$$
\frac{d T}{d r}=\frac{1}{\sqrt{r}} H^{\prime}-\frac{1}{r \sqrt{r}} H
$$

Substituting (4.36) in (4.35) we obtain,

$$
\frac{1}{r^{2}} \frac{d}{d r}\left(r \sqrt{r} H^{\prime}-\frac{1}{2 \sqrt{r}} H\right)-\frac{\alpha}{\sqrt{r}} H=-\beta
$$

The homogeneous equation is:

$$
\frac{1}{r^{2}} \frac{d}{d r}\left(r \sqrt{r} H^{\prime}-\frac{1}{2 \sqrt{r}} H\right)-\frac{\alpha}{\sqrt{r}} H=0
$$

After simplifying and multiplying by $\sqrt{r}$, we obtain

$$
r^{2} H^{\prime \prime}+r H^{\prime}-\left(\alpha r^{2}+\left(\frac{1}{2}\right)^{2}\right) H=0
$$

Eqn. (3.39) is modified Bessel equation of half kind and therefore the solution is: 


$$
H=C_{1} I_{1 / 2}(\sqrt{\alpha} r)+C_{2} K_{1 / 2}(\sqrt{\alpha} r)
$$

According to the characteristics of Bessel's equation, we have $C_{2}=0$. Therefore, the solution to Eqn. (3.40) is:

$$
H=C_{1} I_{1 / 2}(\sqrt{\alpha} r)
$$

The exact solution in terms of $T(r)$ is:

$$
T=C_{1} \frac{I_{1 / 2}(\sqrt{\alpha} r)}{\sqrt{r}}+T_{p}
$$

where, $T_{p}$ is the particular solution and $T_{p}=\frac{\beta}{\alpha}$.

Therefore, the temperature expression considering the superscript * again:

$$
T^{*}\left(r^{*}\right)=C_{1} \frac{I_{1 / 2}\left(\sqrt{\alpha} r^{*}\right)}{\sqrt{r^{*}}}+\frac{\beta}{\alpha}
$$

Considering the boundary condition of Eqn. (3.32-b), and after derivations of Eqn.(3.41) lead to:

$$
\begin{gathered}
\frac{d T^{*}}{d r^{*}}=C_{1}\left(-\frac{I_{1 / 2}\left(\sqrt{\alpha} r^{*}\right)}{2 r^{*} \sqrt{r^{*}}}+\frac{\sqrt{\alpha} I_{3 / 2}\left(\sqrt{\alpha} r^{*}\right)+\frac{1}{2 r^{*}} I_{1 / 2}\left(\sqrt{\alpha} r^{*}\right)}{\sqrt{r^{*}}}\right) \\
C_{1}\left(-0.5 I_{1 / 2}(\sqrt{\alpha})+\sqrt{\alpha} I_{3 / 2}(\sqrt{\alpha})+0.5 I_{1 / 2}(\sqrt{\alpha})\right)=-h_{a}^{*}\left(C_{1} I_{1 / 2}(\sqrt{\alpha})+\frac{\beta}{\alpha}\right) \\
C_{1}=\frac{-h_{a}^{*} \frac{\beta}{\alpha}}{\sqrt{\alpha} I_{3 / 2}(\sqrt{\alpha})+h_{a}^{*} I_{1 / 2}(\sqrt{\alpha})} \\
T^{*}=\frac{-h_{a}^{*} \frac{\beta}{\alpha}}{\sqrt{\alpha} I_{3 / 2}(\sqrt{\alpha})+h_{a}^{*} I_{1 / 2}(\sqrt{\alpha})} \frac{I_{1 / 2}\left(\sqrt{\alpha} r^{*}\right)}{\sqrt{r^{*}}}+\frac{\beta}{\alpha} \\
T^{*}=\frac{\beta}{\alpha}\left[1-\frac{h_{a}^{*}}{\sqrt{\alpha} I_{3 / 2}(\sqrt{\alpha})+h_{a}^{*} I_{1 / 2}(\sqrt{\alpha})} \frac{I_{1 / 2}\left(\sqrt{\alpha} r^{*}\right)}{\sqrt{r^{*}}}\right] \\
T=T_{e}+\frac{\beta}{\alpha}\left[1-\frac{h_{a}^{*}}{\sqrt{\alpha} I_{\frac{3}{2}}(\sqrt{\alpha})+h_{a}^{*} I_{\frac{1}{2}}(\sqrt{\alpha})} \frac{I_{\frac{1}{2}}\left(\sqrt{\alpha} r^{*}\right)}{\sqrt{r^{*}}}\right] *\left(T_{a}-T_{e}\right)
\end{gathered}
$$


Eqn. (3.46) provides the analytical solution for the temperature plot in tissue interior of spherical tissues. The expression shows that the heat fluxes conducted in radial direction toward the surface.

It is worth mentioning that Eqn. (3.27) and Eqn. (3.46) have been developed with assuming thermal insulated core and perfect emitting (emissivity $\approx 1.0$ ) surface, thus the outcome will be versatile and adapt to any condition.

\subsection{Analytical Results}

Applying the analytical solutions discussed in section Chapter 1, the interior temperature field at steady-state can be obtained. The temperature distributions inside homogeneous tissue models are calculated using MATLAB with the thermal and biological parameters of tissues listed in Table 4-4. There is no unarguable reference to evident assumed values to the tissue parameters,

Table 3-2 Thermal and Physical Parameters of Tissues

\begin{tabular}{|c|c|c|c|}
\hline Parameter & Symbol & Value & Unit \\
\hline Thermal Conductivity & $k$ & 0.52 & $\mathrm{~W} /(\mathrm{m} . \mathrm{K})$ \\
\hline Heat Exchange Coefficient & $h_{a}$ & 10 & $\mathrm{~W} /\left(\mathrm{m}^{2} . \mathrm{K}\right)$ \\
\hline Specific Heat (Blood) & $c_{b}$ & 4186 & $\mathrm{~J} /(\mathrm{kg} . \mathrm{K})$ \\
\hline Density (Blood) & $\rho_{b}$ & 1000 & $\mathrm{~kg} / \mathrm{m}^{3}$ \\
\hline Metabolism & $Q_{m}$ & 700 & $\mathrm{~W} / \mathrm{m}^{3}$ \\
\hline Perfusion Rate & $\omega_{b}$ & $5.2 \times 10^{-4}$ & $1 / \mathrm{s}$ \\
\hline Artier Blood Temperature & $T_{a}$ & 310.15 & $\mathrm{~K}$ \\
\hline Environmental Temperature & $T_{e}$ & 300.15 & $\mathrm{~K}$ \\
\hline
\end{tabular}

moreover, former studies are found with discrepant suggestions while assigning values to the featuring parameters. It may be because the biological tissues vary extensively among humans, between organs. Moreover, most of them are in-vitro computed; however, the reasonability of the recommended values is supported by many researchers and will be discussed in Chapter 5 . 
In general body heat is generated due to cell's metabolic activities and partially dissipated by blood perfusion and the remaining transferred to the skin surface. The heat transfer graph in interior of homogeneous cuboid tissue is shown in Figure 4-25, where thermal plots of all the boundary conditions, listed in Table 4-3, are presented. The temperature is plotted as a function of tissue thickness. The plots show that the boundary conditions influence the temperature distribution in tissue inside. Because of the same condition at the skin surface boundary (skin-air interface) in BC-I and BC-II, the curves are well coincident near the skin surface. The similar cases can be seen in BC-I and BC-III near the body core (because the core maintains the constant artery temperature), also in BC-II and BC-IV (because of perfect isolative core), whereas in BC-III and BC-IV at the skin surface (because of perfect emissive surface). A constant temperature $306.75 \mathrm{~K}$ is maintained at skin surface in BC-I and BC-II and $T_{c}=T_{a}=310.15 \mathrm{~K}$ is assumed in BC-I and III. Interestingly, BC-III and IV provide similar temperature graphs because the core should

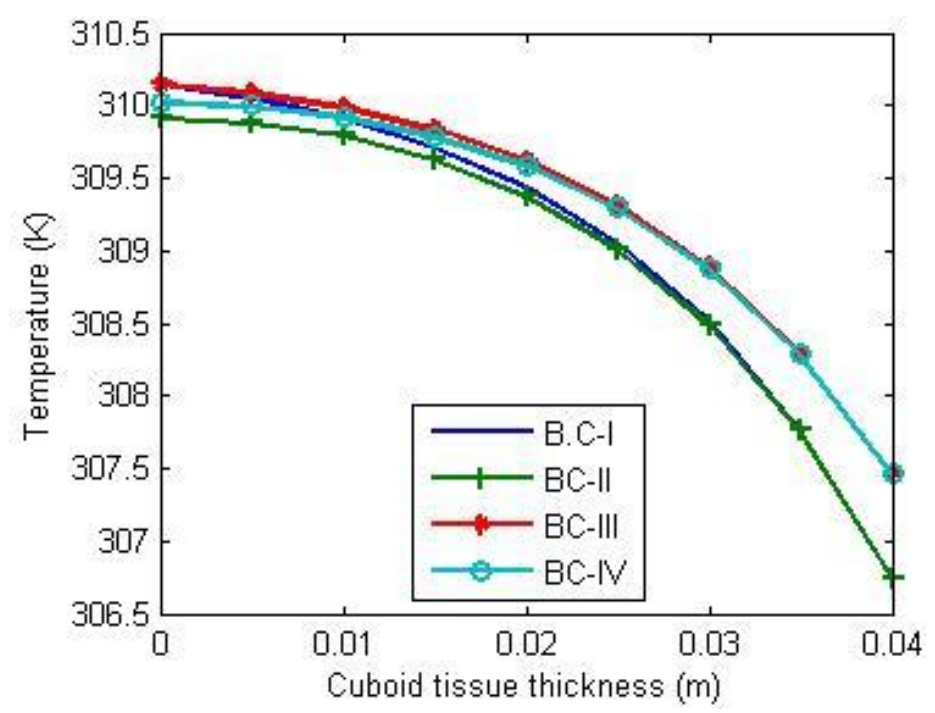

Figure 3-4 Radial temperature flow in homogeneous cuboid tissue from body core toward surface for the boundary conditions in Table 3-1

maintain the temperature equal to or lower than the blood vessel temperature $\left(T_{c} \leq T_{a}\right)$. For unbounded surface temperature, the graphs indicate a temperature difference $\leq 1.5 \mathrm{~K}$ between the core and the surface. On the other hand, for the custom bounded (BC-I) case the heat flow gradient will change with the skin temperature. 
The interior temperature distribution in cylindrical homogeneous living tissues in the resting state is presented in Figure 4-26. This distribution is obtained from the analytical expression

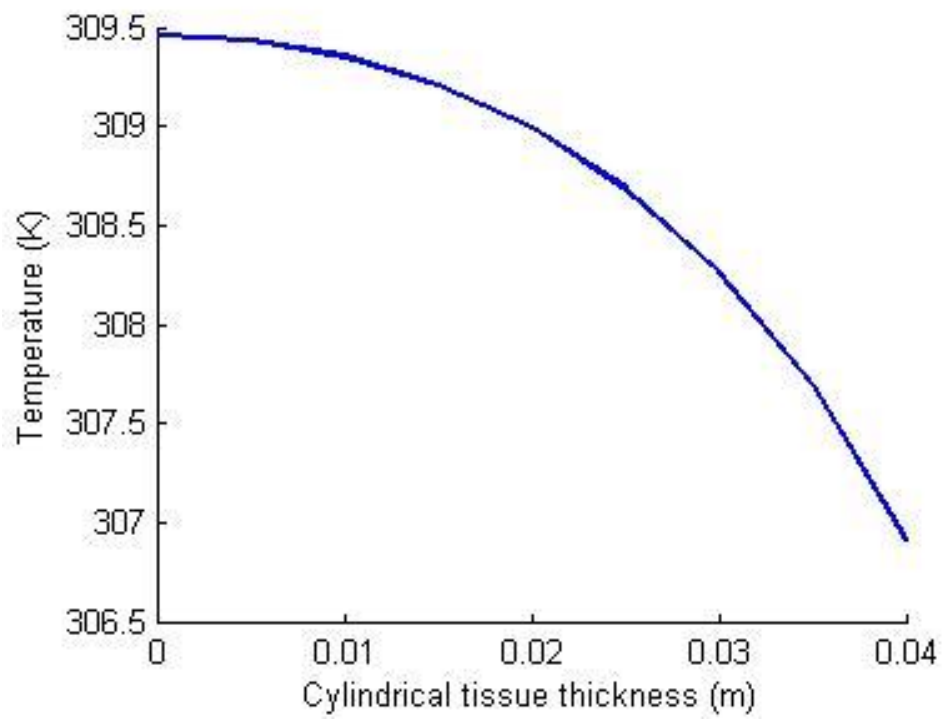

Figure 3-5 Radial temperature flow in homogeneous tubular tissue from body core toward surface for the BC-IV in Table 3-1

derived in Eqn. (3.27). Recall that the thermal expression was deduced for thermal isolated core and purely emissive skin (which is analogues to the BC-IV, in Table 3.1, of Cartesian geometry). The result shows that a tubular organ of radius $4 \mathrm{~cm}$ has a temperature drop of $2.05 \mathrm{~K}$ between center (core) and skin.

Similarly temperature plot on spherical homogeneous tissues obtained from Eqn. (3.46) is shown in Figure 4-27. The curve shows that at steady state the spherical tissues produce a nonlinear thermal diffusion at tissue inside with a center (core) temperature $308.98 \mathrm{~K}$ and peripheral temperature $306.99 \mathrm{~K}$ if the organ has the radius of curvature $4 \mathrm{~cm}$.

\subsection{Comparisons between analytical solutions obtained in different coordinate system}

Even in normothermia or euthermia condition, a wide range of temperatures have been found in healthy people. At specific circumstances, different parts of an individual person's body would have different temperatures. This section will examine the reason why there are different skin temperatures and will discuss how the temperature magnitude can relate to that particular organs exterior and finally, will demonstrate the reasoning of imitating organ's physical geometry (exterior outlook) in developing the physical model. 
The analytical solution of Pennes bioheat equation to compute the temperature fields in cuboid, tubular and curved organs with similar boundary conditions are presented in Eqn. (3.12), (3.27) and (3.46), respectively. The steady-state temperature flows in above three-type organs have been compared to estimate the influences of tissue geometry on the temperature distribution in healthy condition. With the very similar thermal and biological parameters, the interior temperature distributions of homogeneous models of above organs are studied. The spatial temperature distributions over tissue thicknesses have been estimated for $4 \mathrm{~cm}$ thick organs. The result is presented in ; the top curve is the temperature profile in cuboid organ (flat outlook), the middle curve is for tubular, and the bottom one is for spherical organs. It can be seen that the flat

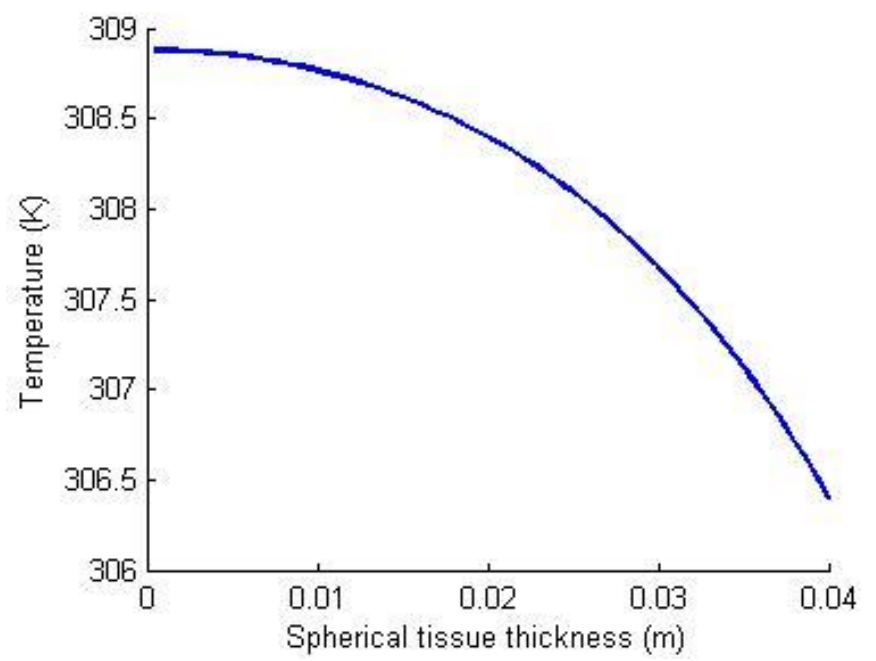

Figure 3-6 Radial temperature flow in homogeneous curved tissue from body core toward surface for the BC-IV in Table 3-1

organ's surface is warmer than the curved organs, to be exact $\approx 0.5 \mathrm{~K}$ warmer than cylindrical tissues and $1.07 \mathrm{~K}$ warmer than spherical organs. This is because of its smaller surface area over which heat losses is taking place. The difference between body core and skin surface temperature is almost $2 \mathrm{~K}$ for each case and dynamic slopes of the graphs are also identical. However, the organ geometry has significant influence on the skin temperature magnitude and establishes the necessity of developing organ models with considering their geometries. 


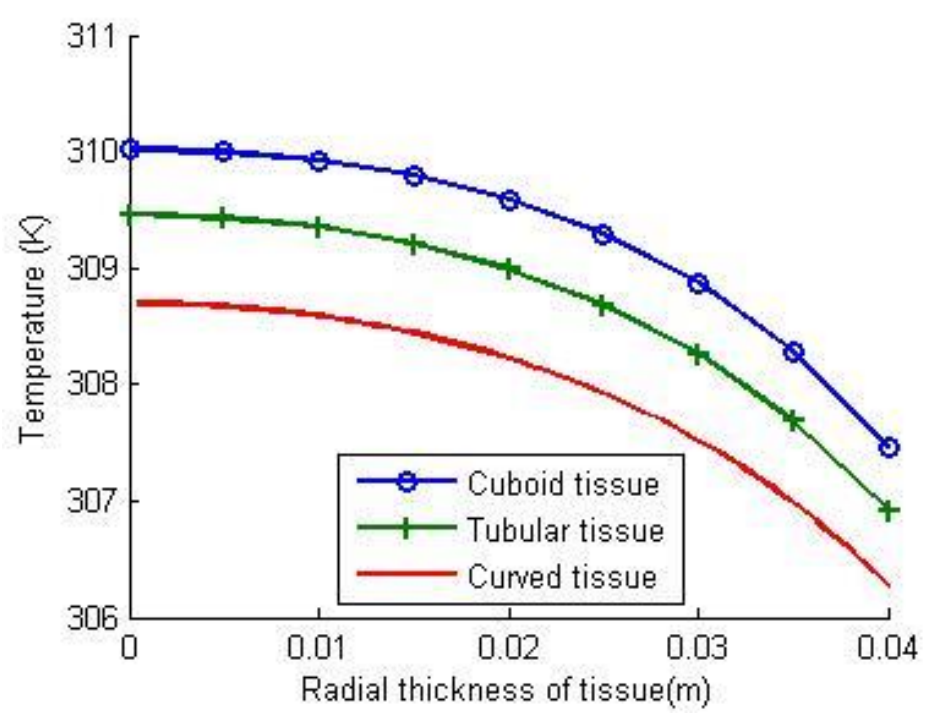

Figure 3-7Comparison of radial temperature flow in homogeneous cuboid, tubular and curved tissues for BC-IV on Table 3-1

\subsection{Numerical Approach}

Above found analytical solutions can determine the temperature distributions inside the living tissues. The temperature plots reveal important information about core temperatures, heat flow gradients and skin temperature magnitudes. However, the analytical process has the following shortcomings:

(i) Temperature probed over simplistic models (homogeneous models)

(ii) Estimating spatial distribution over skin temperature which requires surface temperature expression cannot be achieved from the analytical solution.

(iii) The solutions are not fitted for heterogeneous tissue. Heterogeneity may come not only for multiple tissues layers but also if some tissues become hyperactive and their thermal properties keep altering throughout the live. (This consequence would happen more likely in tumor organs.)

The limitations in the analytical approach may be overcome by the numerical approach. In addition, the ultimate goal of this research is detecting and locating tumors from surface temperature distribution through an inverse approach, which is completely unattainable from the analytical process. Thus the thermal problem will be addressed numerically. Although different numerical approaches (such as Finite Element Method, Finite Difference Method (FDM), and 
Monte-Carlo Method) for analysis of bio-heat problems are available, finite element analyses, which are based on accurate constitutive models, provide the most detailed information on the spatial and temporal distribution of temperature distribution in biological tissues. The objective of the present section was to simulate the bio-heat problems resulting from a healthy organ, using the finite element analysis software COMSOL [71]. This software is a Multiphysics simulation package which has a built-in heat transfer module for live biological tissues. In order to verify the performance of the COMSOL's library model, the very same problem is solved by writing codes in FDM using MATLAB program.

\subsubsection{Finite Difference Method}

The bio-heat model in Eqn. 3.2 with boundaries in Eqn. 3.43 and 3.44 can be solved numerically with FDM for cuboid geometry as shown in , where the bottom, top and lateral (left and right) boundaries are denoted as $\mathrm{BB}, \mathrm{TB}$, and $\mathrm{LB}$ and $\mathrm{RB}$, respectively. Computing interior thermal behaviour requires solving the thermal flow in two directions so the other two lateral sides (front and back) are omitted.

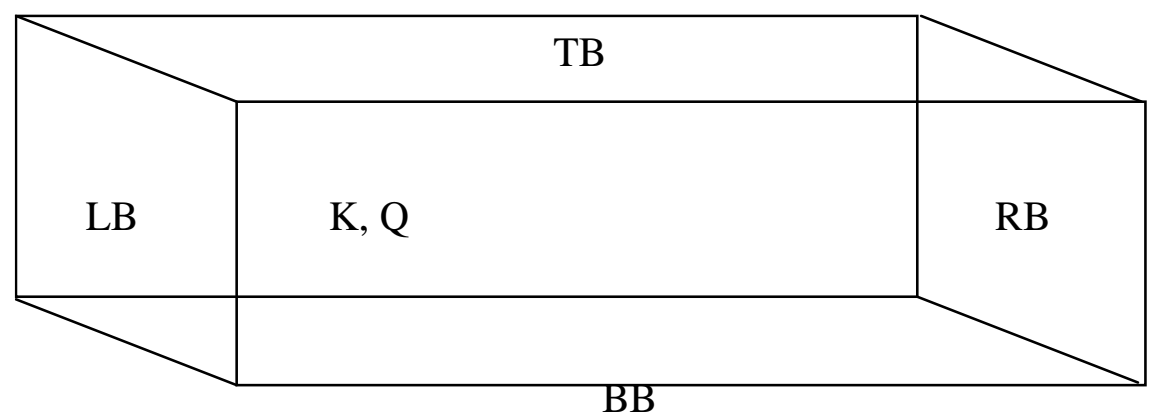

Figure 3-8 Cuboid tissues 3-D domain

Assume the boundaries BB, LB and RB are thermally isolated i.e.

$$
\frac{\partial T}{\partial n}=0
$$

and at skin surface (TB) is entirely emissive i.e.

$$
-k \frac{\partial T}{\partial n}=h_{f}\left(T_{s, a}-T_{e}\right)+\sigma \varepsilon\left(T_{s, a}^{4}-T_{e}^{4}\right)
$$


where $h_{f}$ is the convective heat coefficient, $\sigma$ is the Stefan-Boltzmann constant, $\varepsilon$ is the emissivity of the skin surface, $T_{e}$ is the environment temperature, and $T_{s, a}$ is the skin temperature at a particular point. This boundary condition can be approximated as:

$$
-k \frac{\partial T}{\partial n}=h_{a}\left(T_{s, a}-T_{e}\right)
$$

where $h_{a}$ is the total heat exchange coefficient.

In FDM, the Laplacian in 2D problems can be written as:

$$
\left(\frac{\partial^{2} T}{\partial x^{2}}+\frac{\partial^{2} T}{\partial y^{2}}\right)_{i, j}=\frac{T_{i+1, j}-2 T_{i, j}+T_{i-1, j}}{\Delta^{2}}+\frac{T_{i, j+1}-2 T_{i, j}+T_{i, j-1}}{\Delta^{2}}
$$

where $\Delta x=\Delta y=\Delta$ and in $\mathrm{x}$-direction $i=1,2, \ldots \ldots, n_{x}$ and in $\mathrm{y}$-direction $j=$ $1,2, \ldots \ldots, n_{y}$. Here $i$ and $j$ are the grids in $x$ and $y$ direction, respectively, and $\Delta$ is the grid spacing factor. The number of grids in $x$ and $y$ direction are, respectively, $n_{x}=\frac{x}{\Delta}$ and $n_{y}=\frac{y}{\Delta}$. A sample grid system is shown in .

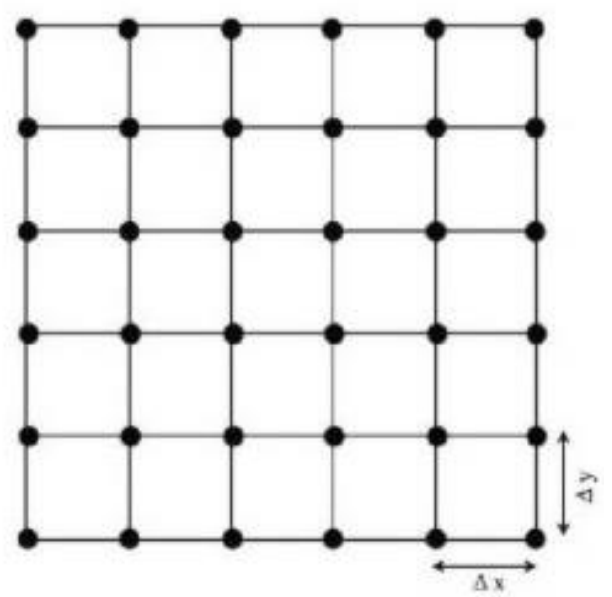

Figure 3-9Finite difference grids

For computing thermal expression for each grid Eqn. (4.2) can be rewritten as:

$$
k\left(\frac{T_{i+1, j}-2 T_{i, j}+T_{i-1, j}}{\Delta^{2}}+\frac{T_{i, j+1}-2 T_{i, j}+T_{i, j-1}}{\Delta^{2}}\right)+\omega_{b} c_{b}\left(T_{a}-T_{i, j}\right)+q_{m}=0
$$

After some algebraic operation Eqn. (3.51) becomes:

$$
T_{i, j}=\frac{k}{4 k+\Delta^{2} \omega_{b} c_{b}}\left(T_{i+1, j}+T_{i-1, j}+T_{i, j-1}+T_{i, j+1}\right)+\frac{\Delta^{2}}{4 k+\Delta^{2} \omega_{b} c_{b}}\left(\omega_{b} c_{b} T_{a}+q_{m}\right)
$$


Except boundaries, temperature at other grid is computed using Eqn. (3.52). Following is the thermal expressions for the boundaries:

At the left lateral boundary LB:

$$
\left(\frac{\partial T}{\partial x}\right)_{1, j}=\frac{T_{2, j}-T_{0, j}}{2 \Delta}=0 \quad j=2,3, \ldots \ldots \ldots, n_{y}-1
$$

Therefore $T_{0, j}=T_{2, j}$ and letting $\beta=4 k+\Delta^{2} \omega_{b} c_{b}$ and $\forall=\omega_{b} c_{b} T_{a}+q_{m}$ and replacing Eqn. (3.52) gives:

$$
T_{1, j}=\frac{k}{\beta}\left(T_{1, j+1}+2 T_{2, j}+T_{1, j-1}\right)+\frac{\Delta^{2}}{\beta} \forall j=2,3, \ldots \ldots \ldots, n_{y}-1
$$

At the right lateral boundaries RB:

$$
T_{n_{x}, j}=\frac{k}{\beta}\left(T_{n_{x}, j+1}+2 T_{n_{x}-1, j}+T_{n_{x}, j-1}\right)+\frac{\Delta^{2}}{\beta} \forall j=2,3 \ldots \ldots, n_{y}-1
$$

Top convective boundary TB:

$$
\begin{gathered}
T_{i, n_{y}}=\frac{k}{\beta+2 \Delta h_{a}}\left(2 T_{i, n_{y}-1}+T_{i+1, n_{y}}+T_{i-1, n_{y}}+\frac{2 \Delta h_{a} T_{e}}{k}\right)+\frac{\Delta^{2}}{\beta+2 \Delta h_{a}} \forall \\
i=2, \ldots \ldots \ldots, n_{x}-1
\end{gathered}
$$

Finally the bottom boundary $(\mathrm{BB})$ condition is:

$$
\left(\frac{\partial T}{\partial y}\right)_{i, 1}=\frac{T_{i, 2}-T_{i, 0}}{2 \Delta}=0 \quad i=2,3, \quad \ldots \ldots, n_{x}-1
$$

Therefore $T_{i, 0}=T_{i, 2}$ and replacing into Eqn. (3.51) gives,

$$
T_{i, 1}=\frac{k}{\beta}\left(T_{i+1, j}+2 T_{i, 2}+T_{i-1,1}\right)+\frac{\Delta^{2}}{\beta} \forall j=2,3, \ldots \ldots \ldots, n_{y}-1
$$

Eqns. through 3.52 to 3.56 are written in MATLAB language to obtain the internal temperature distribution of the cuboid living tissue.

\subsubsection{Finite Element Method}

The COMSOL solver is a powerful and popular numerical analysis software, which works based on the finite element method. The solver is featured with an environment to analyze the heat 
transfer in biological tissues which has been applied to estimate the temperature profiles of different types of living tissues. The COMSOL solver requires developing a physical geometry, identifying domains, defining models and boundaries, selecting analysis type, and growing mesh. A step by step procedure for thermal analysis of rectangular prism homogeneous healthy tissues required for the solver is exemplified by the following:

\section{Geometry Design}

The COMSOL's geometry tool is used to create a cuboid shape shown in Figure 4- 28. The homogeneous tissue geometry is split into three domains. The domains interface line is marked with $\mathrm{AB}$. The purpose of creating the boundary edge $\mathrm{AB}$ is to estimate the temperature flow on that line handily. The dimensions of each domain are labelled on the figure.

\section{Materials Definition:}

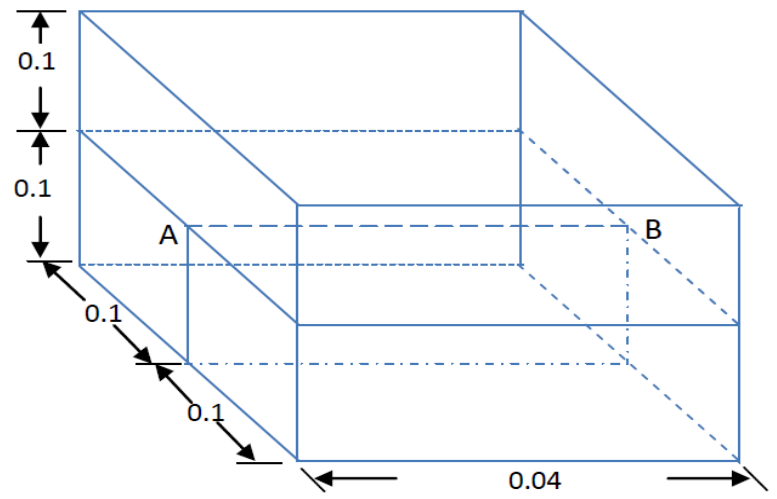

The value for thermal conductivity, density and specific heat of tissue is defined for the Figure 3-10 Dimensions of cuboid model

domains. In this particular problem all domains will be given the same thermal values as specified on Table 4-4.

\section{Physical Model and Boundaries Setting:}

The values for blood density, specific heat, perfusion rate and metabolic heat generation were specified in the developed finite element model. In addition, the values for $T_{c}, h_{a}$ and $T_{s}$ were also defined. 


\section{Analysis Type and Global Definition:}

The steady state analysis was performed using the developed finite element model. For custom designed geometry, parameters can be defined by assigning their physical values. This parameter list may include the dimensions of the geometry, thermal and biological parameters of tissues and blood and thermal values in boundaries.

\section{Mesh Generation:}

3-D thermal model developed for this study is disintegrated into differential elements as shown in. The elements are two shapes - the triangular meshes are mainly seen at the boundaries and everywhere else is tetrahedral. The entire model is divided into approximately 5000tetrahedral elements and 380 triangular elements, on the maximum and minimum element size, element growth rate and resolution are set to $0.05,0.005$ and 0.2 to obtain optimum result.

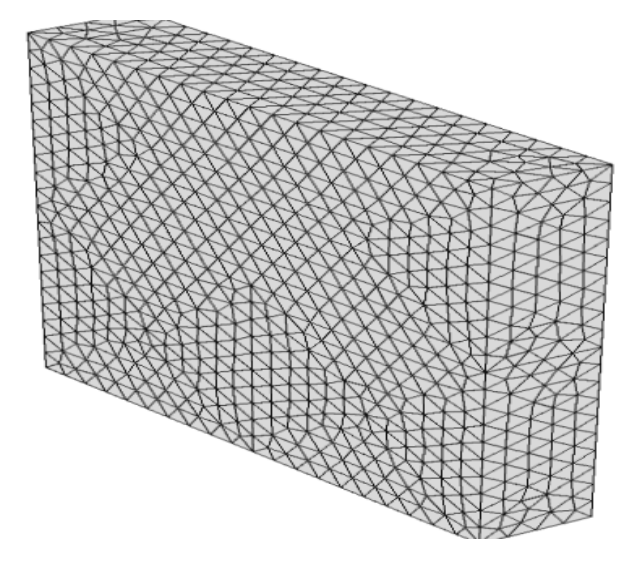

Figure 3-11 Developed finite element model

\subsubsection{Comparison between Analytical and Numerical Results}

Thermal behaviors of homogeneous healthy tissues can be studied both analytically and numerically [using FDM approach and finite element method (FEM) via COMSOL Solver]. Though, all these methods may have some advantages and limitations. Results obtained using these approaches are to be verified to demonstrate their performance and accuracy. 
The thermal results on a cuboid homogeneous tissue model are presented in this section. The analysis has considered the values for the tissue parameters listed in Table 4-4. Though tissue thickness (distance between base of the core layer and the top of the skin surface) is different for different organs, only for comparison's purpose the tissue thickness has been assumed $4 \mathrm{~cm}$ in every case. The FDM approach assumes the domain dimensions of $0.04 \times 0.2 \times 0.2 \mathrm{~m}$ and the grid spacing factor of $1 \mathrm{~mm}$. The number of grids on the $x y$ plane was assumed to be $40 \times 200$ with the convergent factor $\leq 0.0001$ using FEM. Likewise, the maximum and minimum element sizes are $1 \mathrm{~mm}$ and $0.5 \mathrm{~mm}$, respectively, hence, the entire geometry is divided into approximately 500 tetrahedral and 100 triangular boundary elements.

The fundamental thermal behaviour in homogeneous tissues at healthy condition is forecasted separately using the analytical approach and numerical methods (FDM and finite

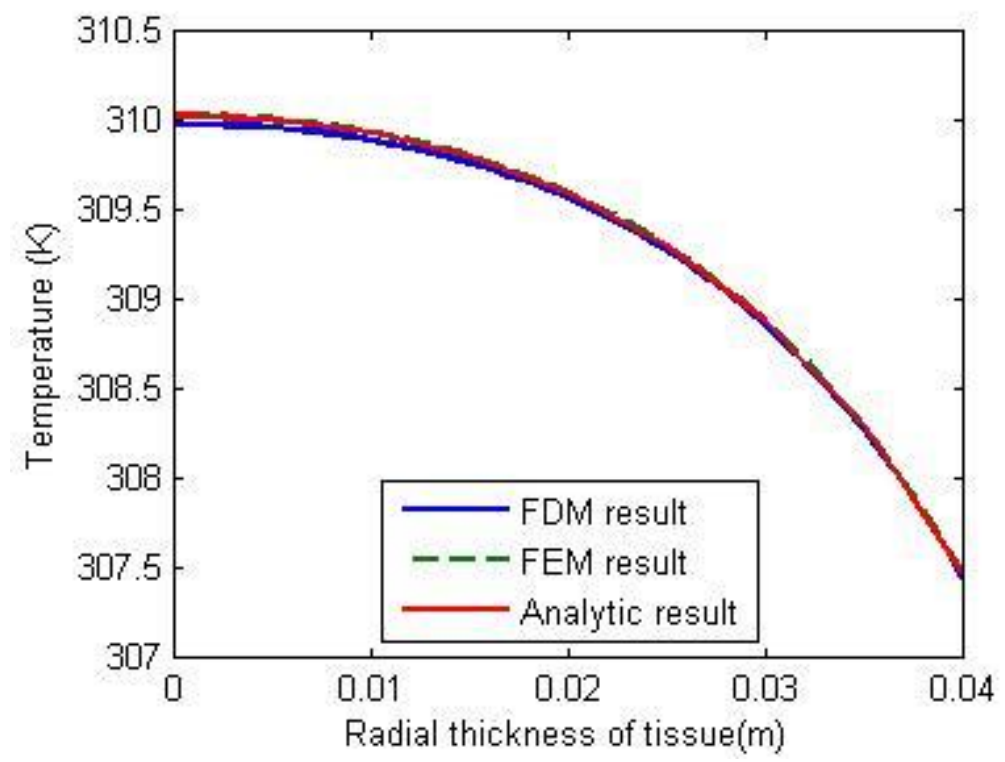

Figure 3-12 Simulated radial temperature flow in homogeneous cuboid tissues using analytical, FDM and FEM: a comparison 
element method). The estimated through the thickness temperature evolution using these three

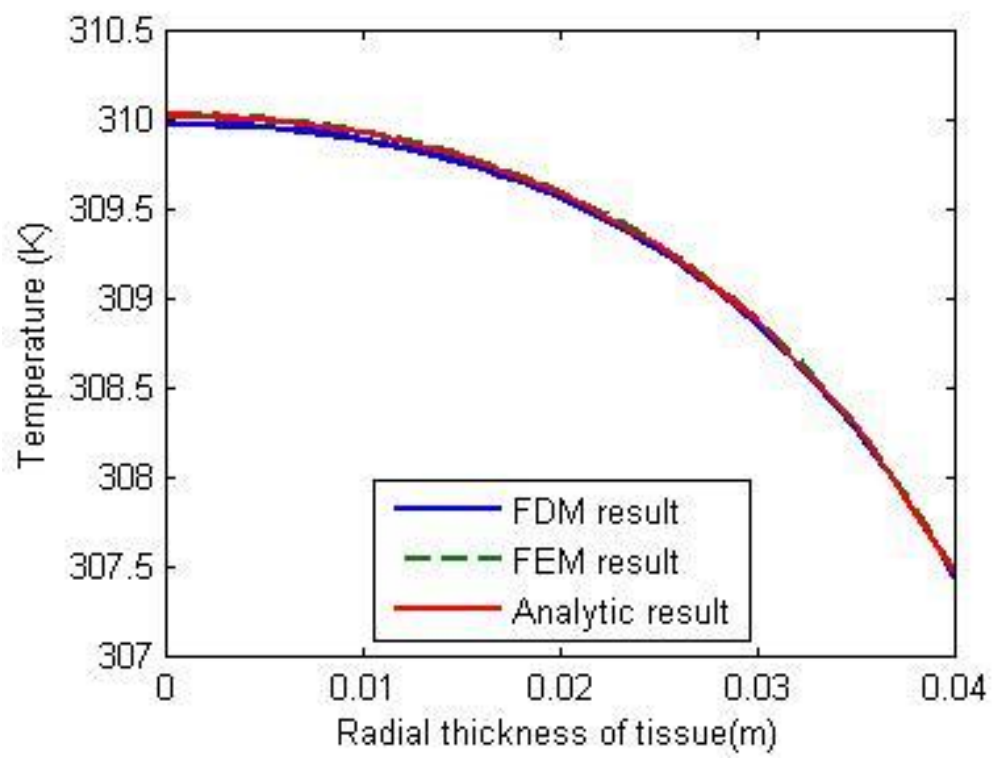

methods is presented in

Figure 4- 29. For a $4 \mathrm{~cm}$ tissue thickness, the graphs show that at skin surface the estimate temperatures have a negligible deviation of $0.0257 \mathrm{~K}$ between FDM and analytical result and $0.0003 \mathrm{~K}$ between finite element and analytical result. However, respective deviations at core end are $0.05 \mathrm{~K}$ and $0.0001 \mathrm{~K}$. The analysis shows that analytical and the finite element forecast is consistent while the FDM estimates discrepant. It may result from non-adaptive definition of finite grid size, accuracy, and/or model dimension.

Therefore, finite element analyses (which are based on accurate constitutive models) of the heat transfer problems of live biological tissues, provide the most detailed information on the spatial and temporal distribution of temperature. Consequently, the finite element analysis using COMSOL Multiphysics is used to predict thermal behavior of biological tissues in our numerical simulation.

\subsection{Homogeneous Finite Element Models}

As discussed in section Chapter 1, FEM is an accurate, effective and stand-alone tool for solving bio-heat problems. The cuboid, cylindrical and spherical homogeneous tissue models of live biological organs were developed using COMSOL Multiphysics software. The finite element results were compared with the analytical ones to validate the developed models. 
The geometries were developed according to the Figure 4-22(cuboid tissue model), Figure 4-23(Cylindrical tissue model) and Figure 4-24(Spherical tissue model). A dimension of $4 \mathrm{~cm} \times$ $20 \mathrm{~cm} \times 20 \mathrm{~cm}$ for the cuboid tissues; radius $4 \mathrm{~cm}$ and height $20 \mathrm{~cm}$ for the cylindrical tissues; and radius $4 \mathrm{~cm}$ for the spherical tissues was assumed. For each case temperature evolution is estimated for tissue thickness of $4 \mathrm{~cm}$ (indicates line AB, see Figure 4-22 to Figure 4-24). The most convenient way to acquire radial temperature flow is splitting the entire domain into several segments as shown in the figures in order to form an inter-domain boundary AB.

After defining thermal and biological properties, the geometries were discretized using solid elements to create their respective mesh as shown in Figure 4-30. The mesh layout of rectangular box model is shown in Figure 4-30(a) where the skin surface was assumed as a heat exchange surface and the remaining five faces are thermally insulated. The finite element cylindrical model has the heat exchange surface at its periphery and both the top and bottom circular surfaces are thermally isolated as shown in Figure 4-30(b). However, the sphere periphery is assumed to exchange heat with environment (see Figure 4-30(c)).

The entire domain of the rectangular pyramidal model was meshed using approximately 27000 tetrahedral and 3500 triangular elements. In the cylindrical tissues, the domain was meshed with more than 200,000 tetrahedral elements and 28,000 triangular elements. Similarly for spherical organs, the tissues were meshed using approximately 5060 tetrahedral and 800 triangular elements. Mesh sensitivity analysis were performed to determine the maximum and minimum element sizes in order to obtain a good resolution in the simulated patterns, and to minimize the computational memory and simulation time. In addition, nodes along the surface area constrained in the normal-translational direction for all three geometries. All other nodes were unconstrained in all directions. A constant temperature of $293.15 \mathrm{~K}$ was applied at the entire section as an initial condition. The maximum and minimum element sizes are chosen as 0.6 and $0.2 \mathrm{~mm}$, respectively. The maximum element aspect ratio is 1.5 .

The steady-state analysis has been performed to estimate the thermal flow throughout the domain. Temperature profile along the AB section inside the tissue is shown in Figure 4-31. The 'black solid' line indicates the analytical curves whereas the 'red dotted' line indicates the finite element results. The plots are shifted in vertical (in temperature) axis where the temperature plot for spherical organs lies under that for the tubular organs and the tubular organ's plot is below of 
the cuboid organ's temperature profile. It can be seen that both approaches (analytical and numerical) produce similar results for the respective tissues.

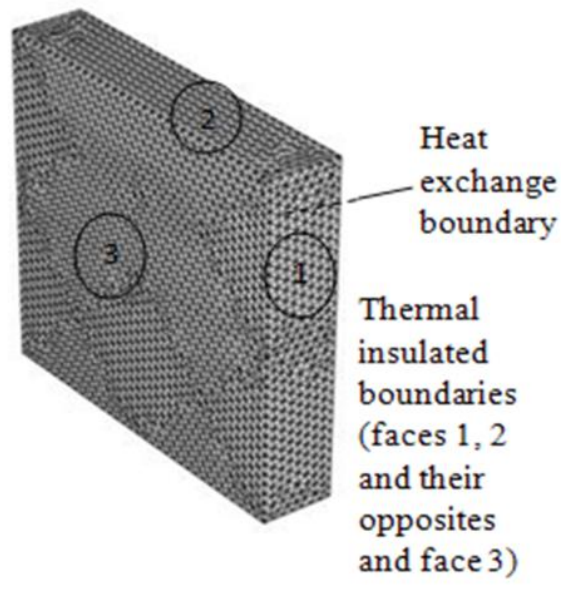

(a)

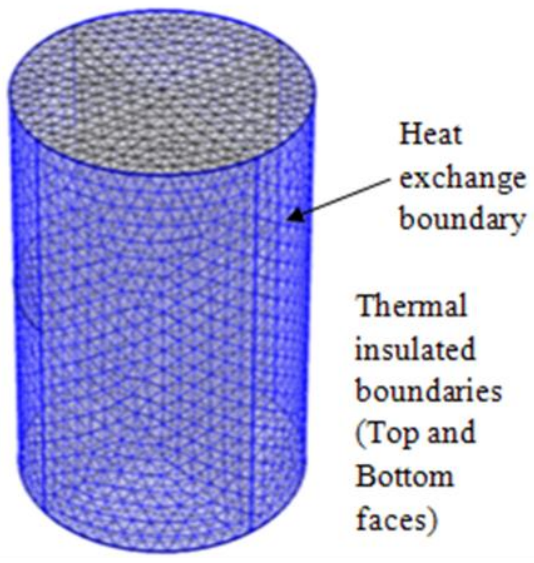

(b)

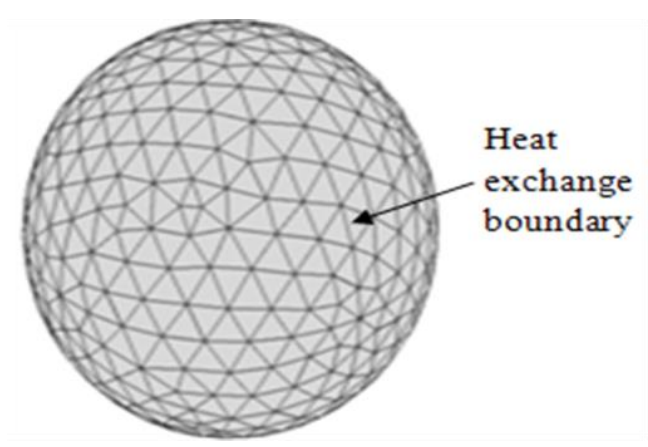

(c)

Figure 3-13 FEM mesh diagrams for (a) cuboid, (b) cylindrical and (c) spherical tissues 


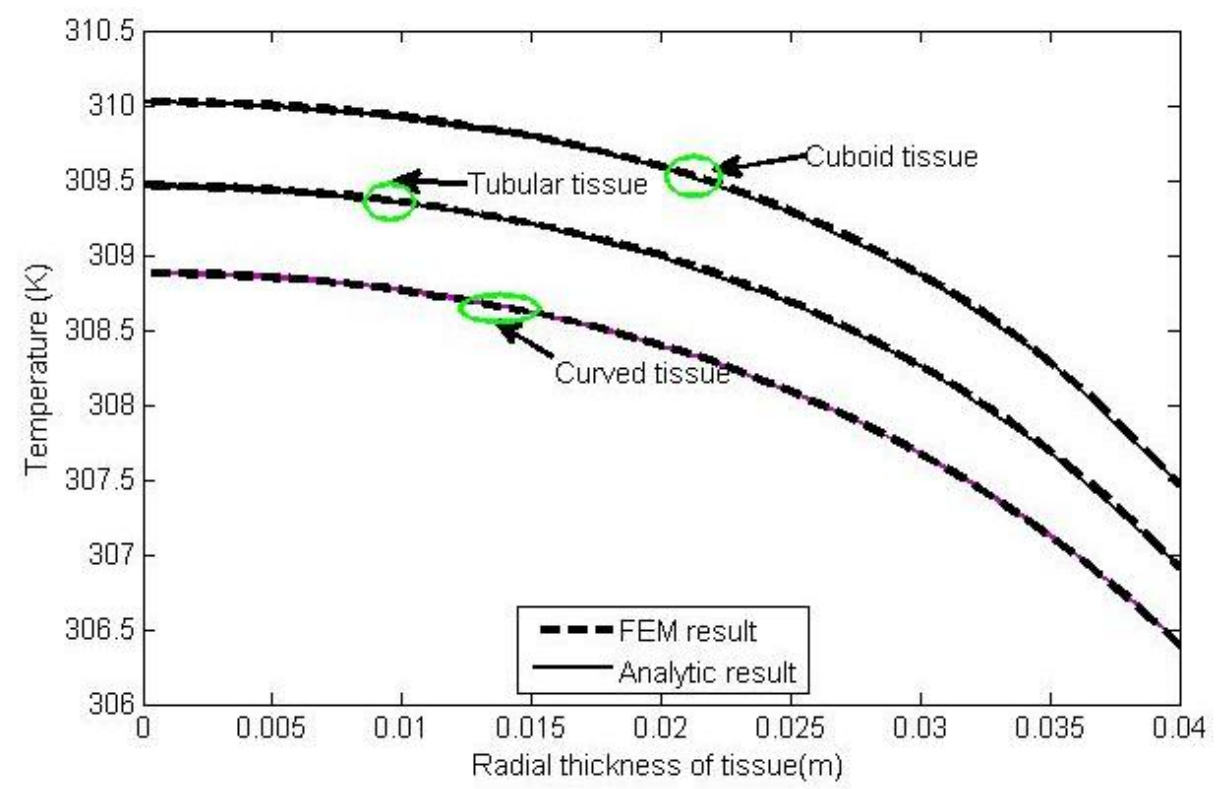

Figure 3-14 Temperature flow in radial direction estimated analytically and using FEM for three different shapes of organs

\subsection{Parametric Study}

The comparative study was performed for a wide range of parameter value. Both the analytical and numerical approaches were validated by parametric analysis to verify how the thermal features of homogeneous chest, forearm and breast models respond to variation of a particular parameter while keeping others unchanged.

The influence of the bio-thermal and physical parameters on the tissue temperature is presented through Figure 4-32 to Figure 4-320. Results are accumulated in two groups; Group 1: influence of thermal and biological parameters, and Group 2: influences of environment and model parameters. The former group includes the parameters which are likely to change in hyperactive tissues. Indeed the latter group, the varying parameters are extracted from ambient conditions and the physics of a patient's specific organ that undergo for diagnosis. The bio-thermal (Group1) parameters includes the metabolic heat rate, conductive thermal coefficient and the blood perfusion rate, while the Group 2 includes the heat exchange rate, ambient temperature and the tissue depth (organ thickness or curvature radius). 
The Figure 4-32 shows the effect of metabolism, conductive heat rate and blood perfusion rate on the temperature distribution of rectangular pyramidal tissues. The Figure 4-32(a) showed

(a)
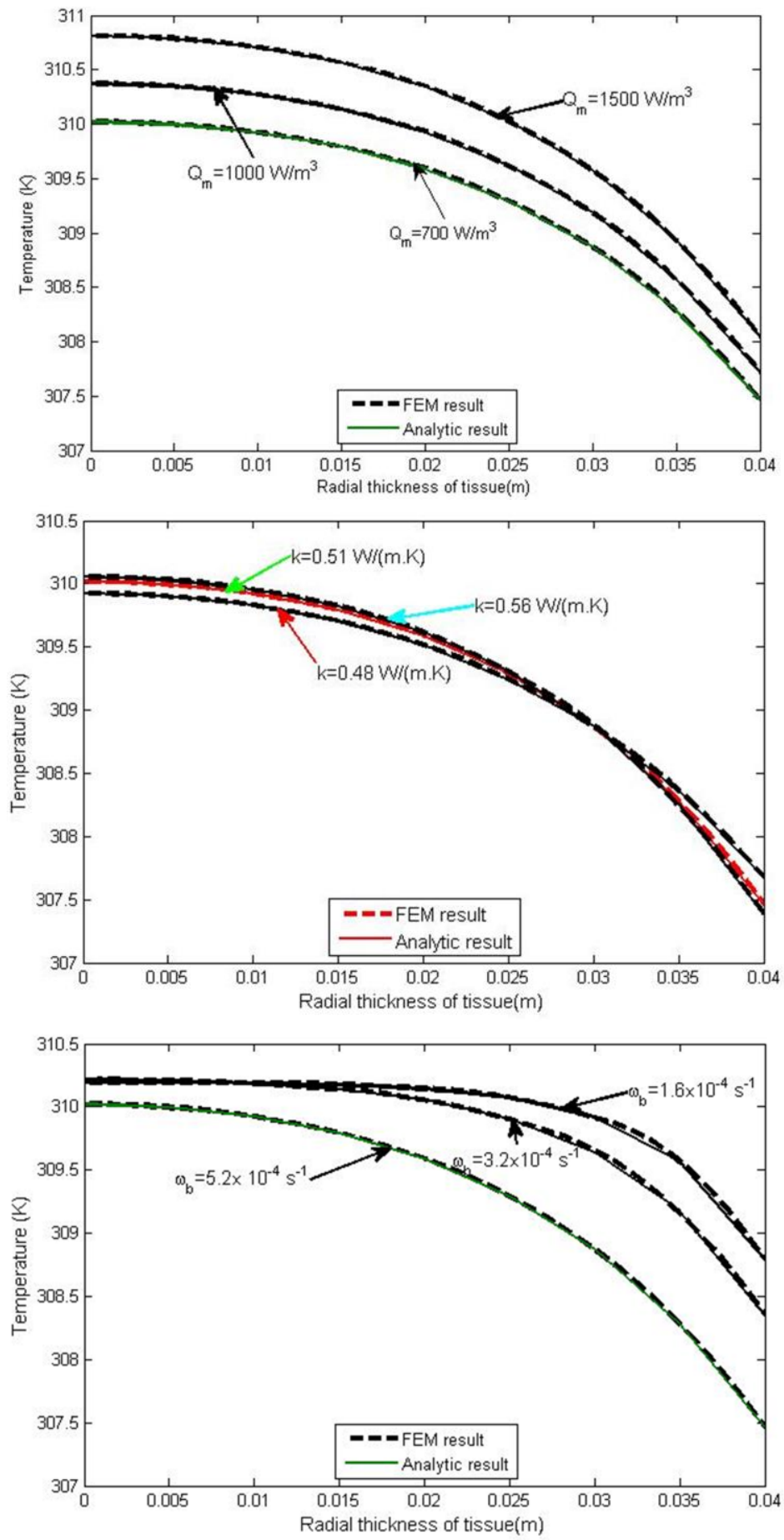

Figure 3-15 Analytical and FEM estimation of biological parameter's effect on radial temperature flow in cuboid tissues; influence of -(a) $\boldsymbol{Q}_{\boldsymbol{m}}$, (b) $\boldsymbol{k}$ and (c) $\boldsymbol{\omega}_{\boldsymbol{b}}$ 
how the tissue temperature has been affected by the change in metabolic heat generation. If the metabolic rate changed from $700 \mathrm{~W} / \mathrm{m}^{3}$ to $1500 \mathrm{~W} / \mathrm{m}^{3}$ the skin temperature increased by almost $0.1 \mathrm{~K}$. Figure 4-32(b) indicates that the thermal conductivity has insignificant effect on the temperature distribution as it varied between 0.48 and $0.55 \mathrm{~W} \mathrm{~m}^{-10} \mathrm{~K}^{-1}$. In comparison with the case of blood perfusion of $\left(\omega=5.4 \times 10^{-4} 1 / \mathrm{s}\right)$, it is obvious that the increase of blood perfusion has a remarkable influence on the surface temperature in living tissues as shown in Figure 4-32(c). The curves in Figure 4-32(c) indicate that the gradient of the temperature variation in radial direction decreases with increasing blood perfusion, which is a result of higher rate of heat distribution caused by the blood perfusion. In addition, the differences between the effects of the higher blood perfusion rates on temperature distributions become smaller. The core temperature approaches to a constant value of $310.15 \mathrm{~K}$. 
(a)

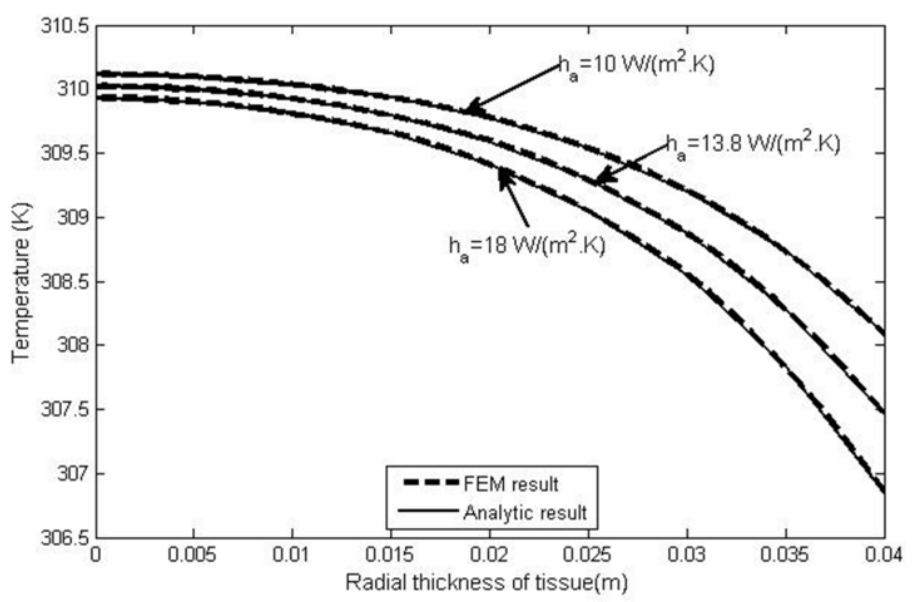

(b)
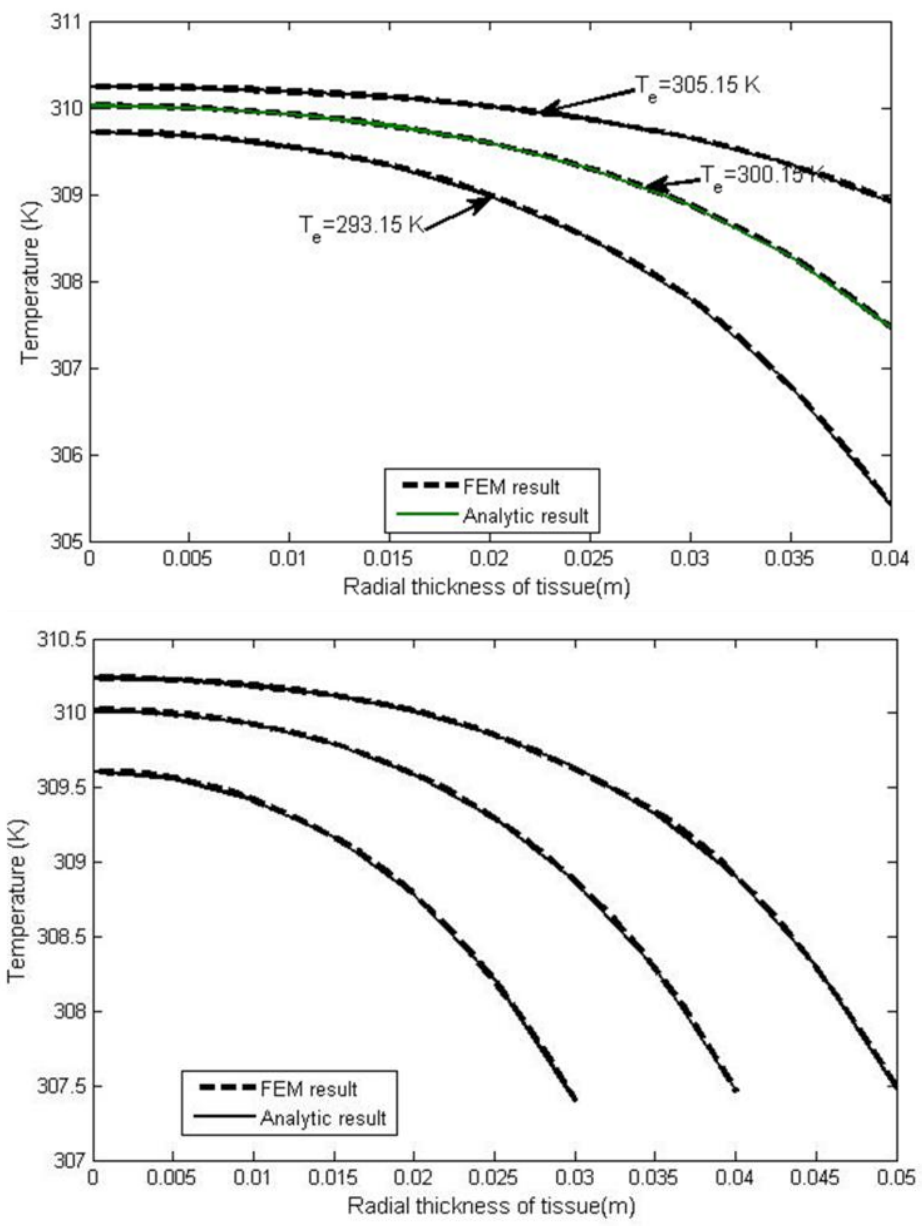

Figure 3-16 Analytical and FEM estimation of ambient and physical parameter's effect on radial temperature flow in cuboid tissues; influence of - (a) $\boldsymbol{h}_{\boldsymbol{a}}$, (b) $\boldsymbol{T}_{\boldsymbol{e}}$, and (c) Tissue thickness

effect of the ambient condition, for example, the heat loss rate and environment temperature on the temperature distribution in rectangular tissue have been presented in Figure 4-33(a) and (b), respectively. Both figures indicate that the ambient conditions have significant effects on the 
surface temperature. Therefore, a controlled environment might play an important role in the hyperthermia diagnosis process. The effect of tissue thickness on the temperature profile is also presented in Figure 4-33(c) where it can be seen that tissue thickness has no significant effect on the surface temperature.

Similar analyses were conducted on the cylindrical and spherical tissues, which are presented in through . and Figure 4-34 show the temperature profile curves for cylindrical model for Group1 and Group 2 parameters, respectively. Temperature profiles for different metabolic rates are presented in (a) for a range between $700 \mathrm{~W} / \mathrm{m}^{3}$ and $1500 \mathrm{~W} / \mathrm{m}^{3}$. If conductive heat transfer rate increases, the body core temperature decreases while the skin temperature increases negligibly, this is evident from (b). Unlike these above two parameters, the higher inflated rate makes the curve saturated at the body core at temperature $310.15 \mathrm{~K}$ with a superficial non-linearity. For increasing perfusion rate, the skin surface becomes hotter which is presented in (c). 
(a)

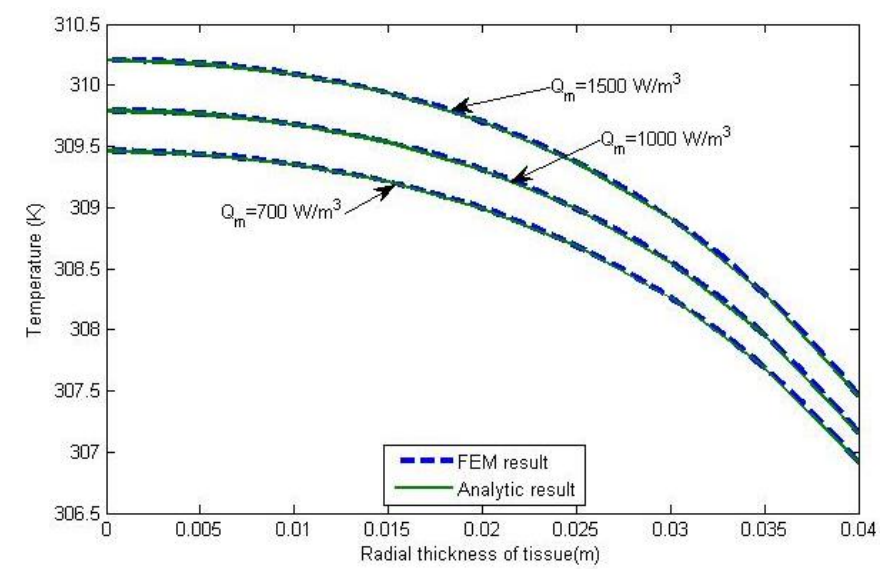

(b)
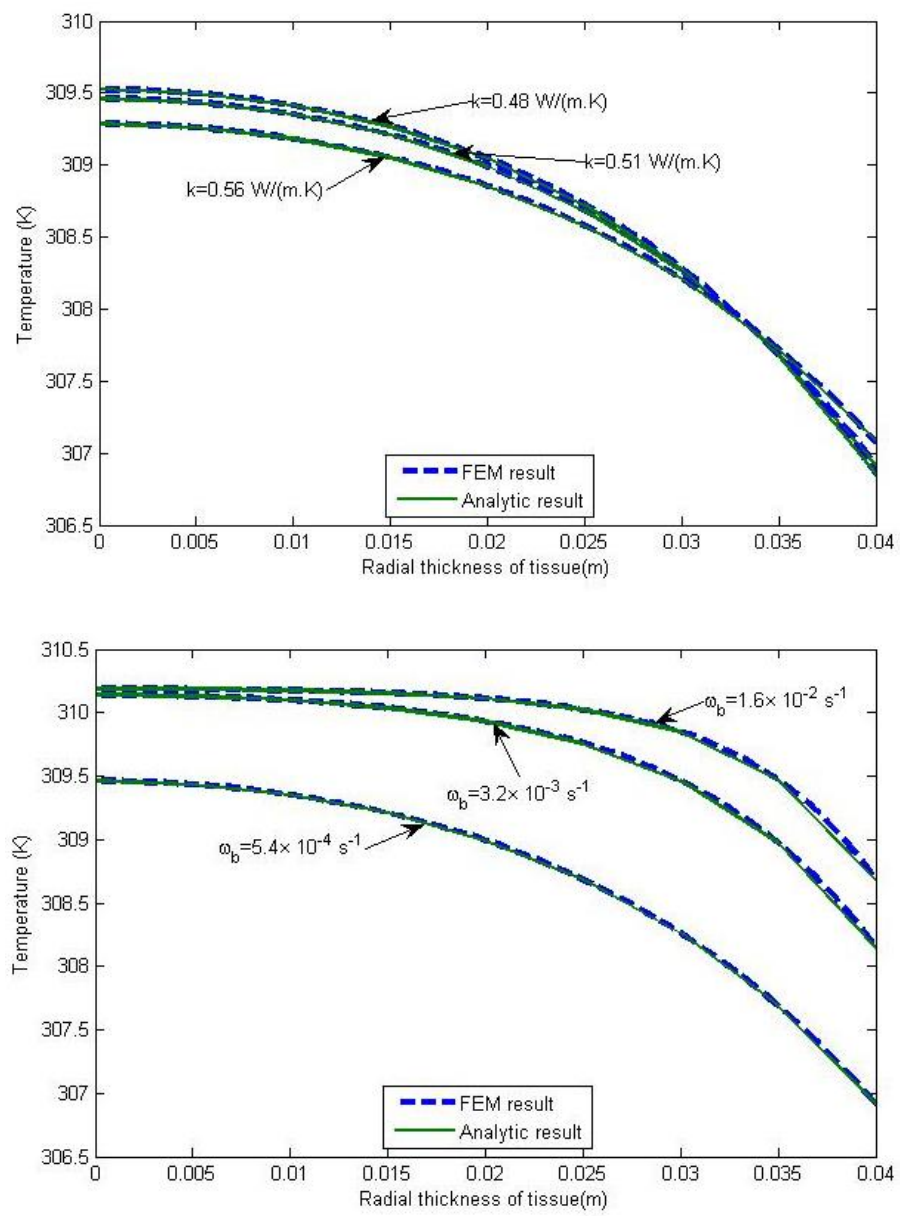

Figure 3-17 Effect of biological parameter on temperature profile in cylindrical tissue influence of (a) $Q_{m}$, (b) $k$ and (c) $\omega_{b}$ 
(a)
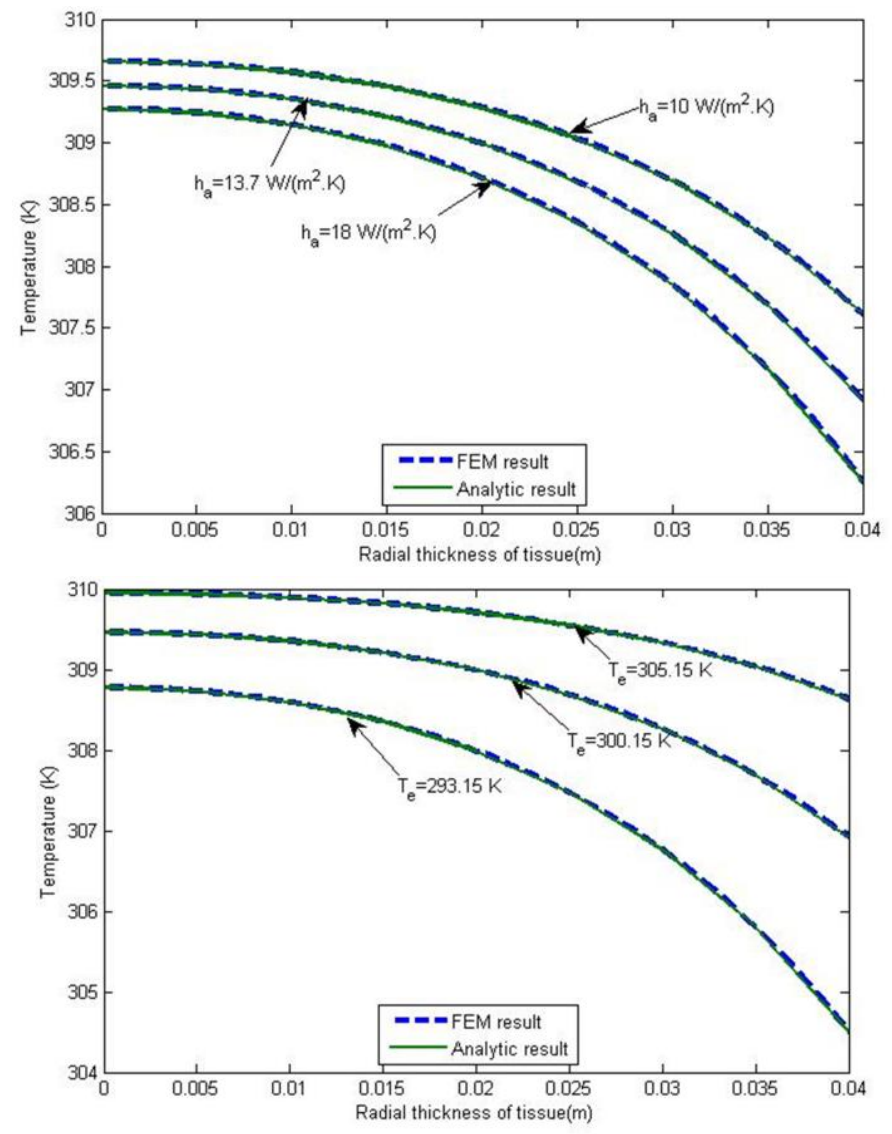

(c)

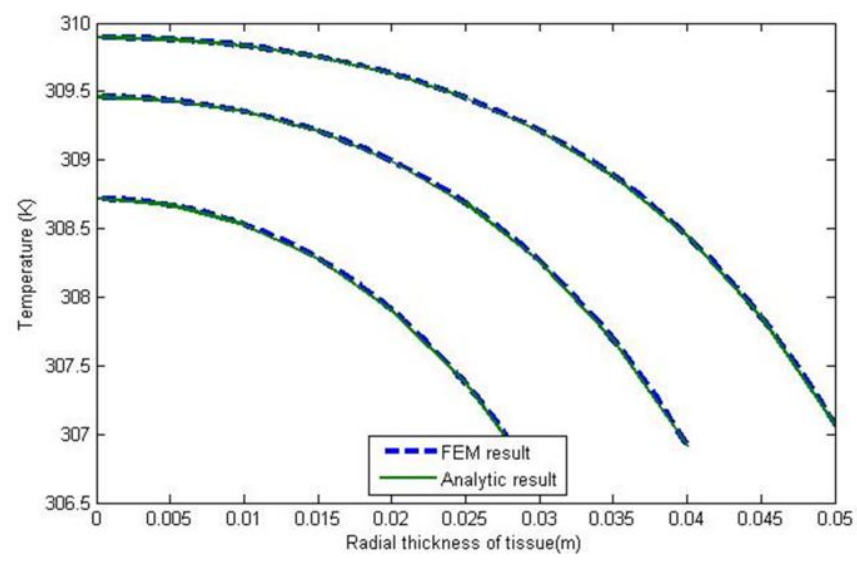

Figure 3-18 Effect of ambient conditions and model thickness on temperature distribution in cylindrical tissues; influence of (a) $h_{a}$, (b) $T_{e}$ and (c) Tissue thickness

The effect of heat loss rate at the skin surface on the temperature profile is shown in Figure 4-34(a). The more vigorously heat transferred to the environment, the less temperature intensity is observed on the surface as well as inside the tissue. The analysis also presents that the ambient temperature 
affects the temperature profile curves [see Figure 4-34(b)]. It can be seen that warm laboratory decreases the interior temperature gradient. Skin temperature could be felt $3 \mathrm{~K}$ higher temperature if the ambient temperature increases from $296.15 \mathrm{~K}$ to $305.15 \mathrm{~K}$. For the same ambient temperature hike the body core reaches about $0.9 \mathrm{~K}$ higher temperatures. Figure 4-34(c) shows that thick tubular organ will produce higher temperature value at body core, which is more than a degree Kelvin for

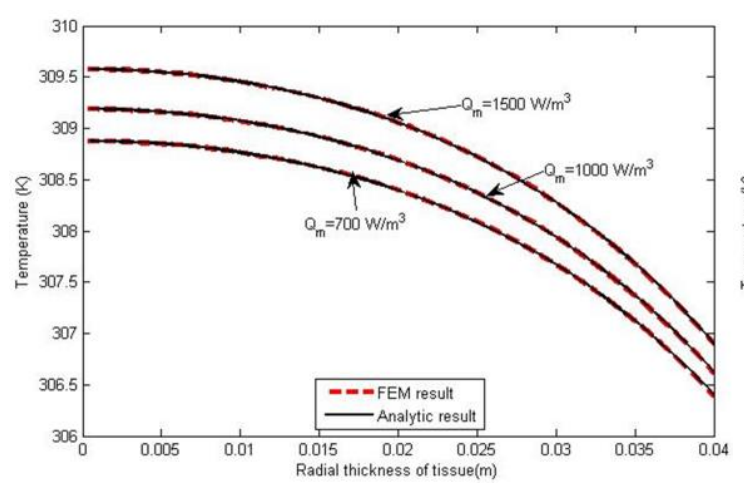

(a)

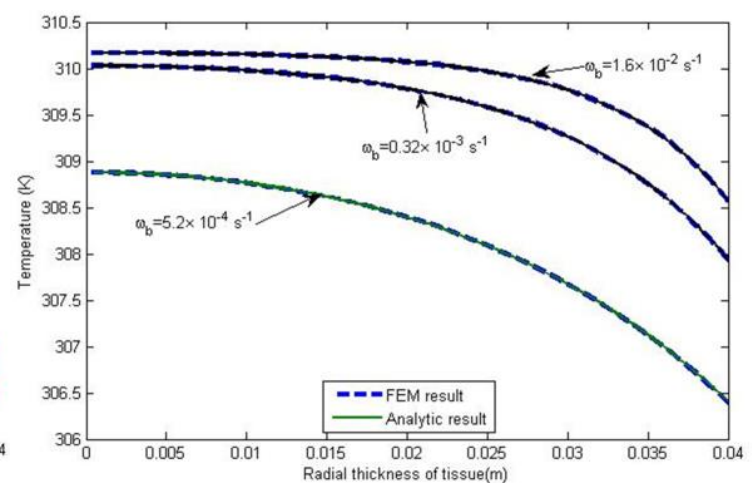

(b)

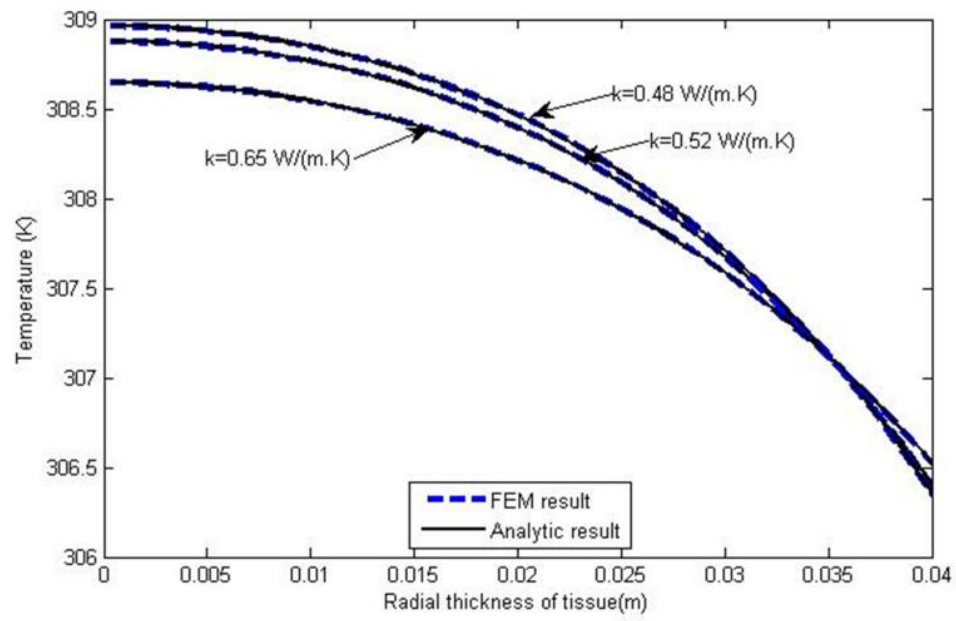

(c)

Figure 3-19 Effect of biological parameter on temperature profile in spherical tissue influence of (a) $Q_{m}$, (b) $k$ and (c) $\omega_{b}$

the organ which has $5 \mathrm{~cm}$ radius than that has $3 \mathrm{~cm}$. However, the effect of organ's thickness may be quite untraceable at the skin surface.

Increasing metabolic rate moves the temperature value up as shown in Figure 4-35(a). The spatial rate of change in temperature with metabolic rate is uniform, at core the rate is $\frac{d T}{d Q}=$ 
$0.025\left(K-m^{3}\right) / W$ while at skin is $\frac{d T}{d Q}=0.0015\left(K-m^{3}\right) / W$. Higher conduction coefficient though increases the core temperature but decreases the skin temperature. The role of conduction heat rate on the temperature flow is presented in Figure 4-35(b). The perfusion rate is a very important function of the in-tissue temperature distribution. The non-linear thermal curve for healthy organ $\left(\omega_{b}=5.4 \times 10^{-4} s^{-1}\right)$ becomes saturated if the perfusion rates increases. The

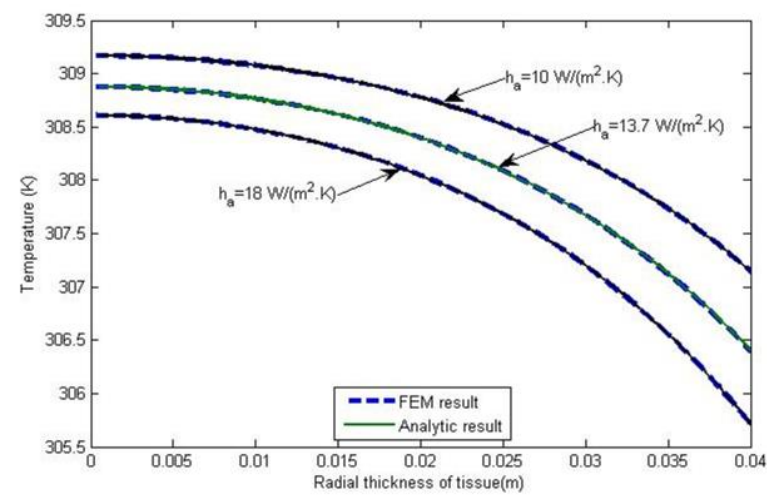

(a)

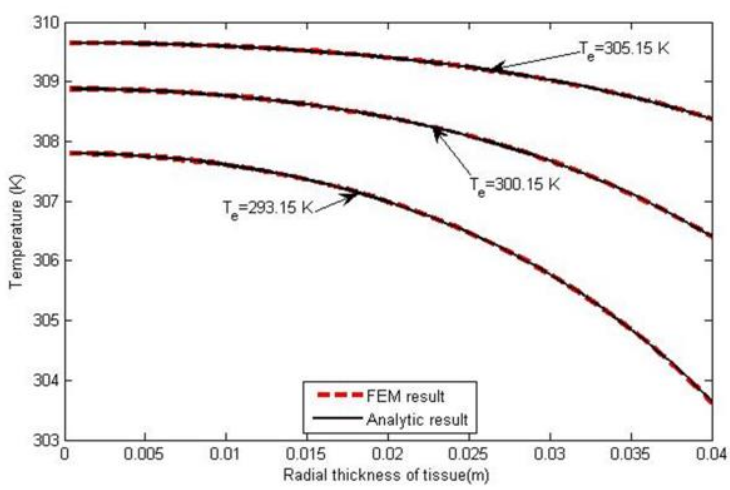

(b)

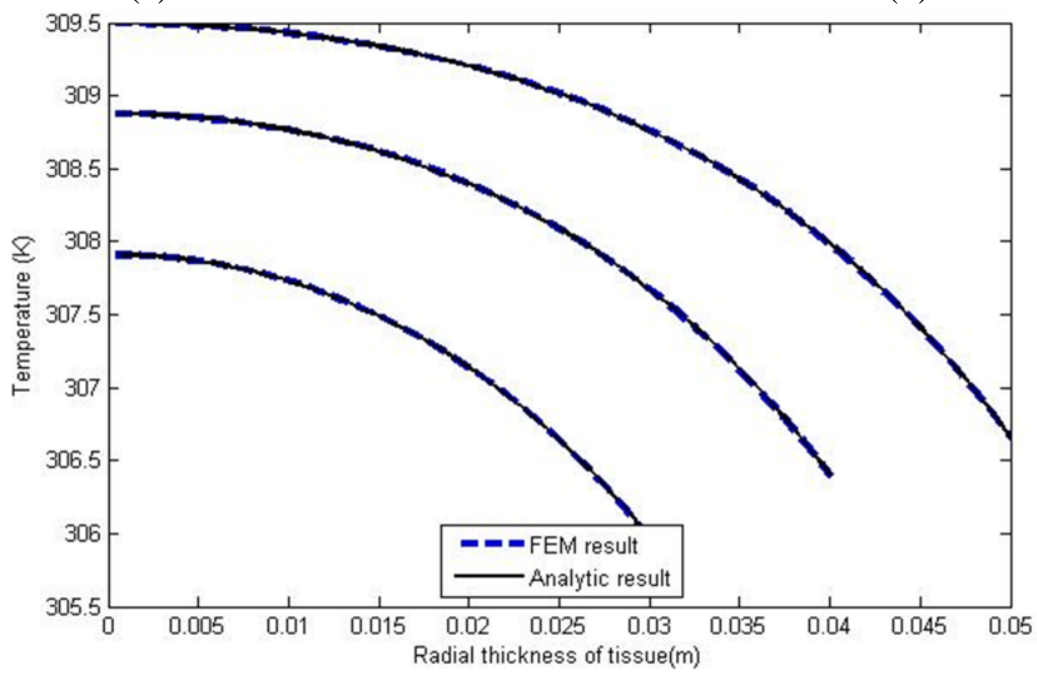

(c)

Figure 3-20 Effect of ambient and model thickness on temperature distribution in spherical tissues- (a) $h_{a}$, (b) $T_{e}$, and (c) Tissue thickness

'saturated part', maintains the constant artery temperature, increases for higher inflammation as shown in Figure 4-35(c).

Thermal image is not only affected by the bio-thermal characters of tissues but also by the thermal condition of the laboratory where the image is acquired. The thermal condition, basically, the heat exchange rate and ambient temperature are two important issues that require investigating. 
The influence of heat exchange rate on the thermal flow is presented in Figure 4-36(a) where the curves show that the exchange coefficient has an inverse effect on the magnitude of temperature and the effect is more sensible at the skin surface than core. Environment temperature also alters the temperature profile in the tissue interior; however, the effect is more pronounced near the skin surface as shown in Figure 4-36(b). The temperature curve varies with radius of curvature of spherical organ which as shown in Figure 4-36(c). It can be seen that the $3 \mathrm{~cm}$ radius organ produces almost $308.15 \mathrm{~K}$ at core and $296.15 \mathrm{~K}$ at skin while the $5 \mathrm{~cm}$ radius organ has $1.5 \mathrm{~K}$ and $0.5 \mathrm{~K}$ higher temperatures at the respective locations.

The above analyses settle the following issues down:

(a) Group 1 and Group 2 parameters have similar effect on the temperature distributions of all models,

(b) The thermal and biological parameters (except the perfusion rate) have relatively stronger effect on the magnitude of core temperature than skin temperature. However, at a certain blood flow rate the temperature curves saturate at the deep region,

(c) Ambient situation affect the thermal images. The skin surface thermal flux controls the magnitude of tissue temperature. The environment temperature also changes the tissue temperature magnitude.

The analytical solutions comply with their respective numerical solutions.

\subsection{Necessity of Anatomical Accurate Models}

Interior thermal profiles on the simplified (homogeneous) models have been discussed in section Chapter 1. It is evident from the estimated radial temperature flows in different organs, shown in Figure 3-7, that organ's shape has important effect on the temperature magnitude and should be mimicked properly while developing the physical model. Referring to the cross-sectional diagrams of several organs (section 0), it is found that they are made on multiple tissue layers where bio-thermal features of each layer are different from others, in particular, the $k, Q_{m}$ and $\omega_{b}$ (Group1 parameters). Individual effects of these parameters have been discussed in Figure 3.15, 3.17 and 3.19 to demonstrate their notable influences on tissue's heat transfer features. For simplistic model, the variations in bio-thermal features of different tissue types that build the organ is omitted and assumed that the organ is homogeneous (made up with single type of tissues). 
Consequently, thermal estimations over simplified models could give pessimistic results and may not be applicable for clinical purpose. To resolve this inaccuracy, the realistic or anatomic-accurate models have been developed in Chapter 4, mimicking organ's true anatomy accordingly (see Figure 4.6, 4.8 and 4.10). Temperature computations over both simplistic and realistic models are presented in Figure 4-37, where the temperature flow in the chest, forearm and breast models are presented, respectively, in top, middle and bottom window and the temperature flow in simplified and realistic models of each organ are shown together. Figure 4-37(a) shows that accurate chest model estimates at least $1 \mathrm{~K}$ lower temperature on the surface than simple mode. It can also be seen that the continuity of heat flow graph for realistic model falls sharply at $0.33 \mathrm{~m}$ from where the fat layer start, it is because of the lower thermal conductivity of fat layer than the muscle layer (see Table 5.7). For the true forearm model the similar discontinuity shows up at $0.35 \mathrm{~m}$ [Figure 437(b)]. In the case of the realistic breast model, bends happen [Figure 4-37(c)] at the interface of two different tissue layers for example the fat-muscle, fat-lobule and fat-skin interface. However, for simplified models the graphs remain continuous because of uniform bio-thermal values 

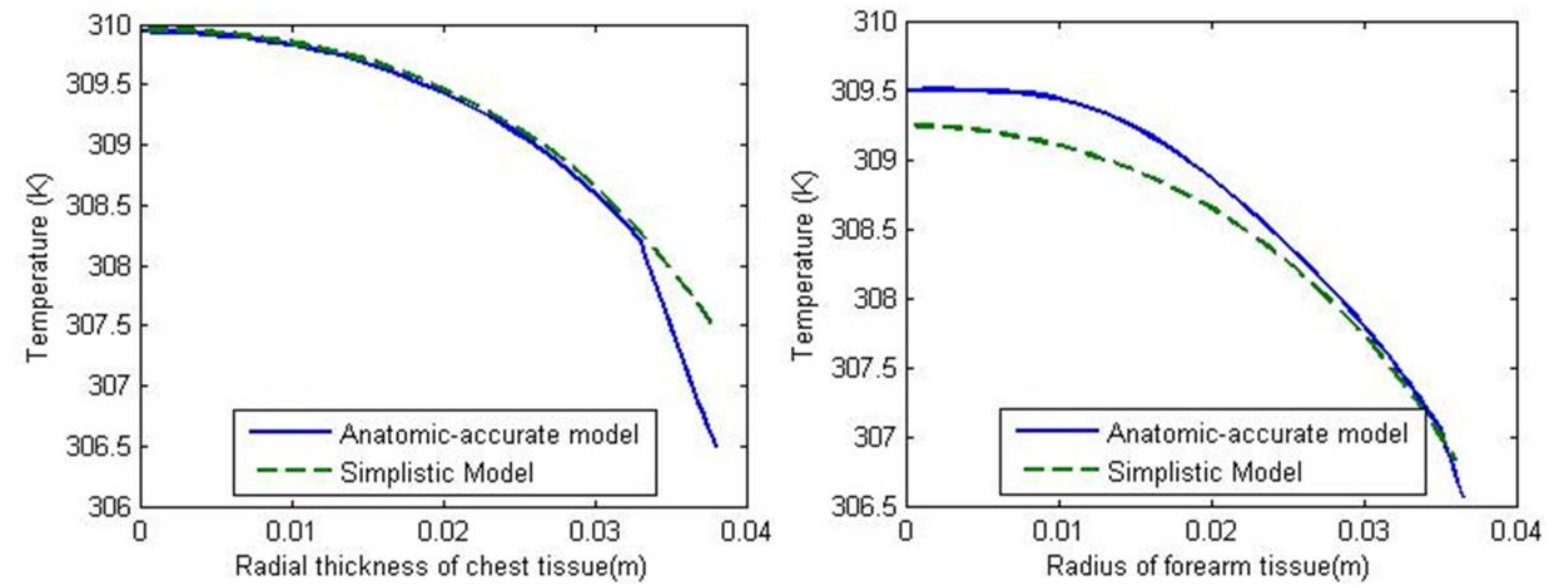

(b)

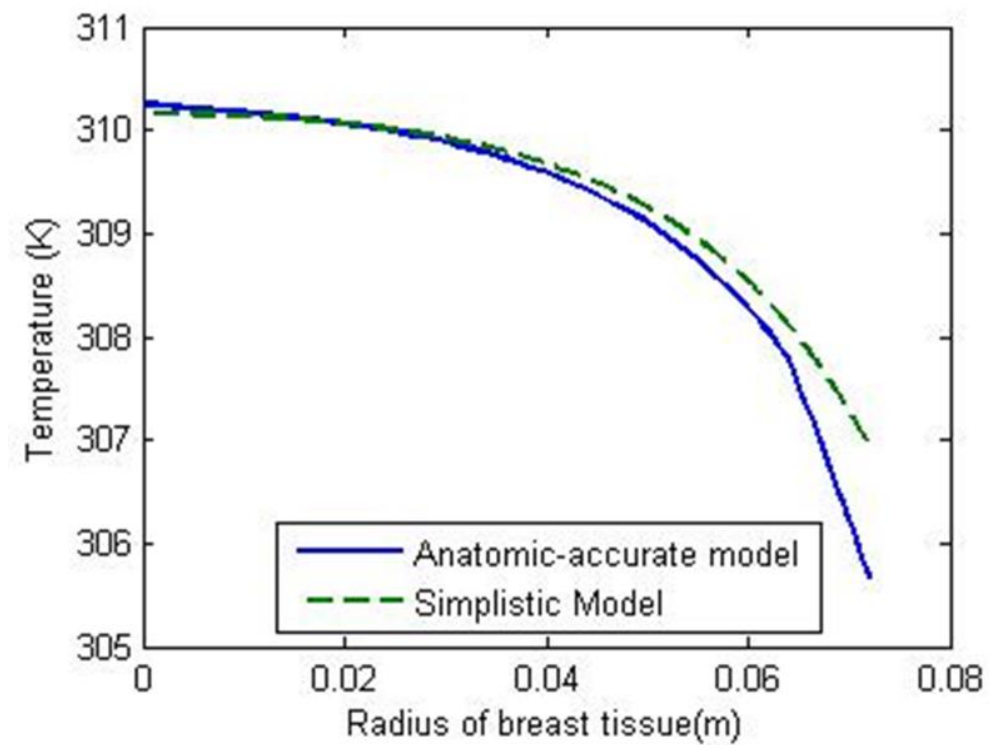

(c)

Figure 3-21 Estimation of radial temperature flow over anatomic-accurate (realistic) and simplistic (homogeneous) models for-(a) chest tissues, (b) forearm tissues, and (c) breast tissues

throughout the entire domain. As the purpose of the research is developing a novel methodology for parameterizing tumor parameters for clinic application from thermogram, therefore from now on the subsequent study will emphasize the computation of thermal features on anatomical accurate models.

\subsection{Summary}

Inner tissue thermal features of a healthy organ have been studied analytically and numerically. The expression of radial temperature profiles has been derived for cuboid, cylindrical and spherical organs by solving the one dimensional Pennes' bio-heat equation at steady-state. The 
analytical solution expressed with trigonometric function for cuboid model and with the Bessel's function for cylindrical and spherical models. As a consequence, the accurate initial temperature field can be easily achieved with respect to the various transient analysis and calculation of heat transfer in living tissues.

The results obtained by the analytical analysis were compared to the numerical solution to demonstrate the suitability of numerical approach. The analysis also shows how an organ's geometry controls the temperature texture. The analysis facilitates finding the effect of thermal conductivity, heat generation rate, blood perfusion and heat exchange coefficient on the temperature distribution which can provide a good knowledge of thermal behaviour of living tissues. This information can be applied to measure the thermal parameters, to reconstruct the temperature field with the help of optimization method and also can be extended for the thermal diagnosis and hyperthermia treatment. 


\section{Chapter 4 Physical Models of Non-homogeneous Tissues}

\subsection{Introduction}

For valid thermal data, a bio-heat transfer problem of living tissues has to be solved on a physical model closely resembled to the anatomic structure. Not only the biological and thermal parameters e.g. the thermal conductivity, perfusion, heat convection and radiation of the biological tissues but also the shape of exterior and anatomic structure of interior of an organ influence the temperature distributions. Therefore comprehensive thermal models of the human body's external organs should come up with the study of thermal mechanism being addressed on models where both the anatomical structures and physiological characteristics of the organ should be compiled in developing the models.

According to Figure 1- 12, the external organs of the human body generally have three different outlooks, namely - the arm, forearm, finger, and leg have tubular (cylindrical) exteriors, the chest (male) and back have rectangular prism outlook and the breast (female) and buttock have hemispherical shapes. The shape of an organ assists the reliable computation for convection and radiation heat losses. On the other hand the knowledge of tissue interior contributes to figure out the conductive heat flow, the metabolic heat production and the perfusion heat loss; these vary significantly among tissue types. Therefore the thermal models of external organs in the human body will be featured with - a standard (cuboid, cylindrical and hemispherical) structure, and heterogeneity (inhomogeneity). Developments of the anatomically accurate 2D and 3D thermal models of the above three-type organs have been discussed in this chapter. A brief review of the existing thermal models has also been deliberated.

\subsection{Organ's Anatomy}

Organ's shape has an influential consequence on the thermal behavior. Let alone the internal tissue compositions and their respective thermal behavior, the prominent heat flow direction(s) and the convection and radiation heat losses surface overall area contribute to the regional thermal distribution, consequently attributes an uneven thermal graph on the body outward. In this study, the superficial organs (or a segment on an organ) in human body has categorized into three groups, with each group typically composed into four tissue layers (bone, muscle, fat and skin). The only exception is the female breast, which has an extra lobule (mammary) tissue layer underneath the 
fat. The basic idea of this study is to model organs mimicking the true anatomy i.e. the organ's

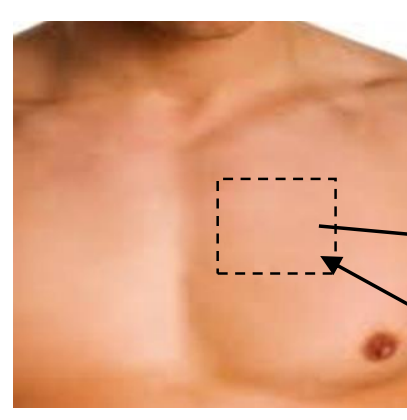

(a) Male chest outlook

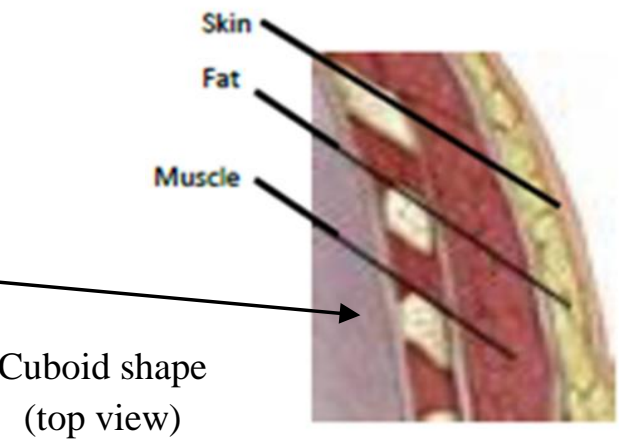

(b) Male chest cross-section

Figure 4-1 Male chest anatomy

shape and the inhomogeneity. To summarize, the proposed model will be built on a bone-filled muscle (body core) topped, one after another, with muscle, fat and skin tissues and will have the structure like a box, a cylinder or a hemisphere.

Firstly, development of the thermal model for a flat organ (e.g. the male chest) has been discussed. The outer-appearance and cross-sectional diagram of male chest is shown in Figure 41. The highlighted (rectangular or square) area on the chest, as shown in (a), has been modeled. The chest constituent tissue layers underneath the selected part are revealed in (b). It is seen that the chest is founded on a rib-filled intercostal muscle tissues which is encrusted with the subsequent muscle, fat and superficial skin layers. The intercostal muscle layer is termed as the body core which is assumed to have a constant temperature $310.15 \mathrm{~K}$. On top of the body core a thick, fan-shaped muscle is situated which is called the pectoral major tissue. Underneath the pectoral major tissues there is a thin, triangular muscle layer called the pectoral minor. The subcutaneous tissue (hypodermis) layer is right above the pectoral major tissue. The hypodermis mainly consists of loose connective tissues, lobules of fat, blood vessels and nerves. The fat layer is covered by a skin layer, which consists of dermis and epidermis layers. Together the fat, dermis and epidermis layer is called the skin which is the principal organ for dissipating heat. The human body dissipates approximately $85 \%$ of its heat loss through the skin under normal environmental conditions. The interface between the epidermis and dermis is extremely irregular and consists of a succession of papillae, or finger-like projections. The skins emissivity is around 0.97 which makes it almost perfect as a radiator and absorber. 


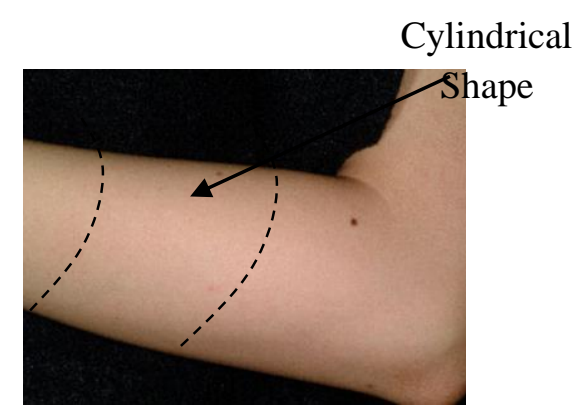

(a) Forearm outlook

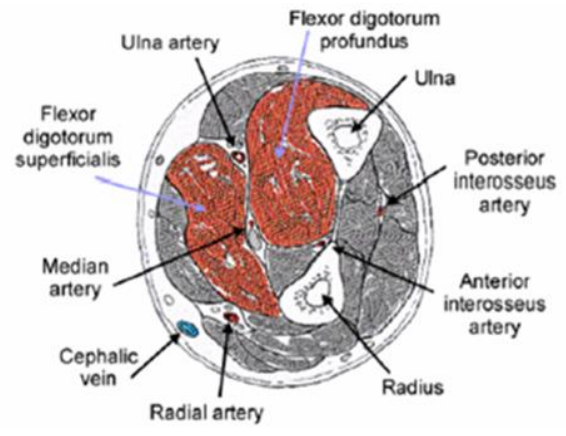

(b) Forearm cross-section

Figure 4.2 Forearm anatomy

Secondly, the thermal behavior of a tubular organ (e.g. the forearm) has been examined. The Figure 4.2 presents the shape and cross-section of an adult male forearm. It is evident that the selected part of forearm, as shown in (a), can be modeled by concentric cylinders of three realistic tissue layers (muscle, fat and skin). Indeed the larger cylindrical surface of forearm contributes the higher amount of the convection and radiation heat losses than the flat organs. The heterogeneity of the forearm tissues is depicted in (b). The cross-section includes bones (e. g. radius and ulna), arteries (e.g. radius artery, ulna artery, posterior interosseus artery, anterior interosseus artery, and median artery), muscles (e.g. flexor digotorum profunds, flexor digontorum superficial and soft tissues) and cephalic vein. Like the chest, the skin over the forearm has three layers-the epidermis, the dermis, and the fat lobule. The average diameter of an adult forearm is roughly 70 $\mathrm{mm}$.

Finally, the female breast has been considered to compute the thermal feature of hemispherical-like organs. The study launches considering the natural, non-deformed breast because of the nearly hemispherical structure, which is shown in Figure 4.3(a). The circular dotted lines around the breast base and above the nipple explain the reasoning of assuming a halfspherical model. The perception of the model domains could be understood from the internal edifice, which is illustrated in Figure 4.3(b). The interior of the breast is made up of three major tissue types, parenchymal (functional) or glandular tissue, fibrous tissue and fat. The parenchymal tissue of the breast is compartmentalized into 15-20 lobes, which is where milk production occurs. The lobes are separated by fibrous walls and can be further divided into smaller compartments called lobules. The lobules are connected to the nipple by a network of ducts. The ducts begin as tiny ductules at the lobules, which merge together as they near the nipple into larger and larger 
ducts. The ducts and lobules are contained and supported by fibrous or connective tissue. All this glandular (lobes and ducts) and fibrous tissue can be grouped together as fibroglandular tissue. Outside the fibroglandular tissue is a layer of adipose or fatty tissue. The proportion of fibroglandular to adipose tissue varies between individuals and with age. There is always a significant proportion of fatty tissue, with even small breasts rarely being composed of less than a third of fat. After menopause, the fibroglandular tissue atrophies, resulting in a much higher proportion of fat to fibroglandular tissue.

Beneath the breast is a large muscle, the pectoral major (pectoral muscle), which separates the breast from the rest of the body. The pectoral muscle is the muscle that is contracted when the hands are placed on the hips and the elbows thrown back. The pectoral minor is found between the pectoral major and the ribs. The whole of the breast is supported by fibrous strands called Coopers ligaments, which are attached to the muscle wall. Coopers Ligaments strengthen the breast significantly, and weakening of these ligaments can cause the breast to sag. The whole of the breast is also supplied with blood vessels. In this thesis we assume that the breast has not been augmented

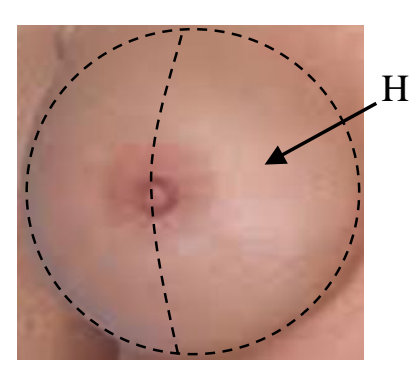

(a) Breast outlook
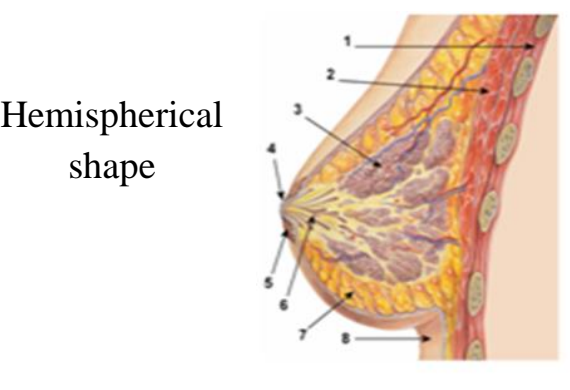

(b) Breast cross-section
1. Chest wall

2. Pectoralis

muscles

3. Lobules

4. Nipple

5. Are ola

6. Milk duct

7. Fatty tissue

8. Skin

Figure 4.3 Breast anatomy

with silicone implants. There are two other major tissue types which we will need to consider. The skin is a layer of highly elastic tissue and is generally 1-2 millimeters thick.

\subsection{Anatomical-accurate Model}

An anatomically accurate model of an organ is a precondition for estimating the exact thermal behavior. At the past, most studies were limited to the thermal analysis of the breast because of its likelihood of developing cancer or tumor. A comprehensive breast model had been presented by Osman and Afify [72] with taking into consideration metabolic heat production, tissue perfusion with capillary blood, arterial and venous blood thermal interactions. However, the 
cross-sectional view of the female breast shown in Figure 4.3(b) presents that the breast is heterogeneous and the thickness of layers are uneven. In addition, the hemispherical geometry could be found only for an ideal and non-deformed breast in supine condition only. The breast cross-section [Figure 4.3 (b)] shows that the breast comprises of two fat layers - a subcutaneous fat layer just under the skin and another fat layer sandwiched between the gland (lobule) layer and the deep muscle layer. The glandular layer is beneath the subcutaneous fat layer whereas the muscle layer (pectoral major and minor) lies close to the thoracic wall just below the retromammary bursa (not shown in the figure). The breast is developed on a deep muscle (pectoral muscle) layer and on the chest wall (muscle layer). The overall breast is covered with a superficial skin layer except the nipple at the vertex which is surrounded by areola tissues.

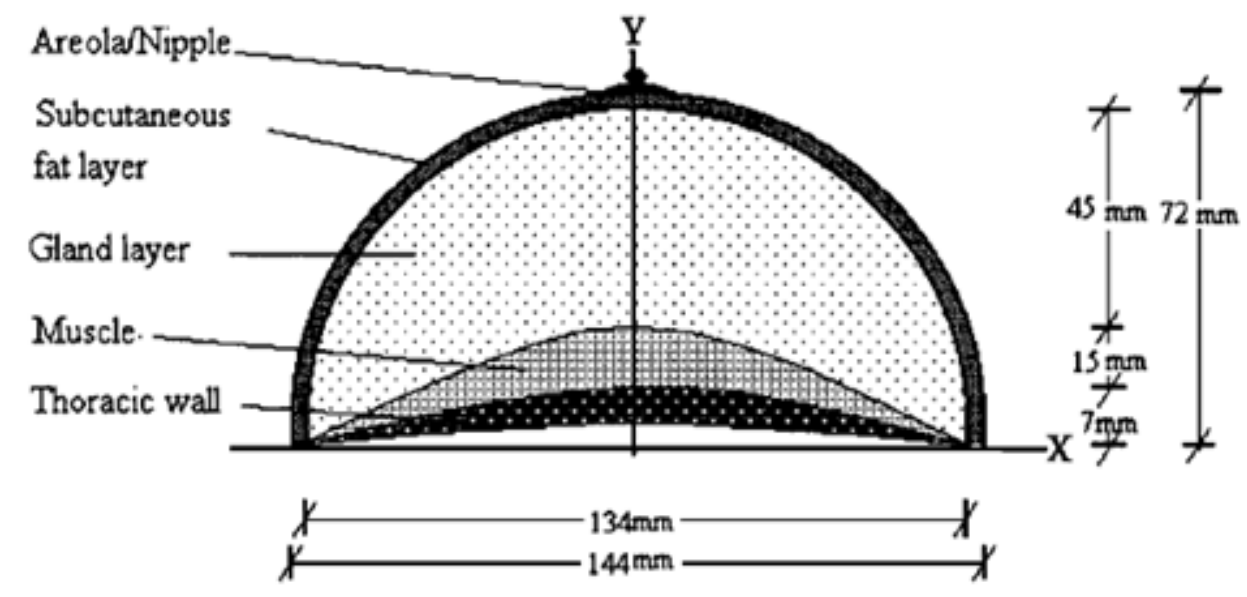

Figure 4.42D model of a female breast

NM Sudharsan et al. [58] proposed a hemispherical breast model considering thickness irregularity pretending to closer to the breast anatomy. The two-dimensional model of the authors is depicted in Figure 4.4. The authors had assumed the breast to be of hemispherical shape with a $5 \mathrm{~mm}$ of subcutaneous layer, followed by a gland and muscle layer of varying thickness. Polynomial functions were defined to compute the thicknesses of layers though the fat layer between the gland and muscle layers was overlooked. It is also worth mentioning that the nipple tissue was not considered in the model. In spite of having the above-mentioned limitations, modern studies have applied the breast model in Figure 4.4 for thermal analysis on breast. 
Though the thermal feature of forearm was first addressed analytically in the early twenties but so far no model was proposed to estimate the surface temperature on the forearm or such

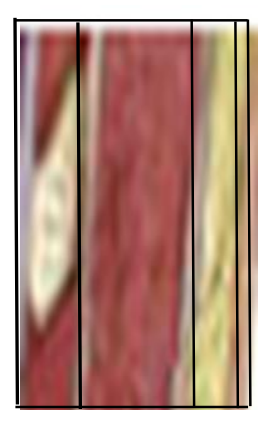

(a) Chest tissue domains

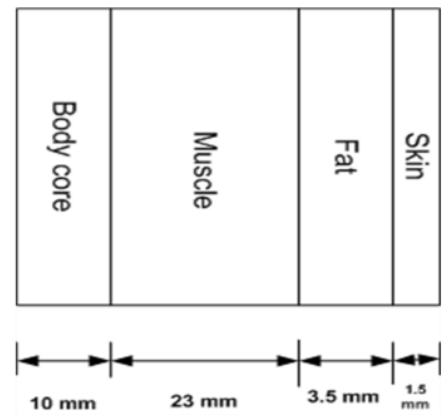

(b) Tissue thicknesses in different domains

Figure 4.5 Male chest - (a) Cross-sectional diagram, and (b) Development of chest model base tubular organs. Fortunately, a latest study conducted by JP Agnelli et al. [14] has developed a rectangular box model to forecast the thermal behavior for an embedded tumor. The authors developed a simple, ideal and two-domain model regardless any real human organ anatomy.

Having looked at the thermal imaging modalities (as discussed in section Chapter 1), it is seen that infrared thermogram provides superficial information as an indication of tissue functionalities in terms of heat radiation and dissipation. Relating the thermal images with tissue functionalities demands a realistic physical model of the human body organ which could be attained by developing a segmental model according to the physical structure and tissue heterogeneity.

\subsubsection{The Chest (Flat Organ) Model}

Generally a portion on a human's back or male chest has almost a flat building. The study considers a rectangular specimen from the male chest, which is marked with the dotted area in Figure 4-1(a). Though the thickness of the chest wall varies among individuals due to their food habits and physical activities, the average chest wall overall thickness of $40-45$ year, $1.7-1.75 \mathrm{~m}$ and $65-70 \mathrm{~kg}$ adult male is between 3.6 and $3.8 \mathrm{~cm}$ [73] which includes three layers such as muscle, fat and skin. Lagoundoye et al. [74] showed that the subcutaneous fat layer could be 0 $6 \mathrm{~mm}$ thick. Taking the sample chest flesh as shown in Figure 4-1(b), resize the thickness of the chest wall to $3.8 \mathrm{~cm}$ which provides a proportion to the muscle layer $3.3 \mathrm{~cm}$ and the fat layer of $3.5 \mathrm{~mm}$ which is covered with the relatively thin skin layer of $1.5 \mathrm{~mm}$. Off the muscle layer, it 
seems obvious that the ribs are located within the bottom $10 \mathrm{~mm}$ region. The chest physical model thus developed consists of four layers - the body core, the muscle tissue, the fat soft tissue and the skin as shown in Figure 4.5. The thickness is assumed to be uniform and the thickness of each layer is labelled on the figure.

A three-dimension physical model of the male chest segment is shown in Figure 4.6. The dimensions of the model are $10 \mathrm{~cm}(\mathrm{~L}) \times 10 \mathrm{~cm}(\mathrm{~W}) \times 3.8 \mathrm{~cm}(\mathrm{D})$ which has a surface area 100 $\mathrm{cm}^{2}$. The body core is mainly built with the pectoral minor tissue and bone (rib) tissues. However, the influence of bone (ribs) tissues on the thermal feature is neglected because the body core is assumed to maintain a constant temperature $310.15 \mathrm{~K}$. The muscle layer, built with pectoral major tissues, is the main component of the model.

\subsubsection{The Forearm (Tubular Organ) Model}

Apart from the flat organs, maximum external organs in the human body have a cylindrical surface and more interestingly some of them don't hold any complex functioning inner organs, thus appealed a great interest to the previous bio-thermal researchers. The study has analyzed the thermal behavior of male's forearm tissues.

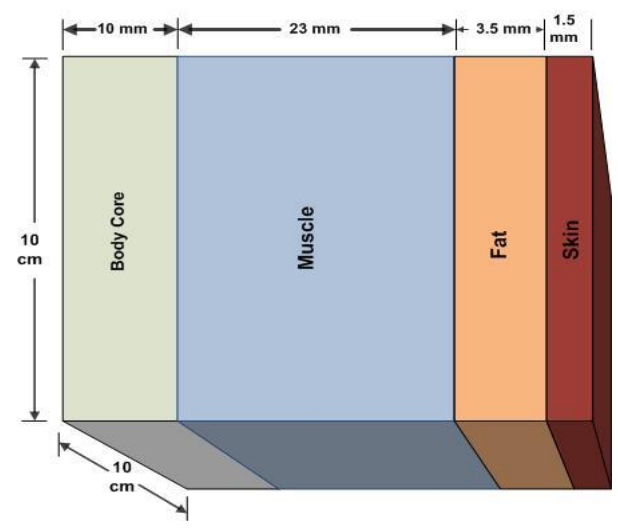

Figure 4.6 Three-dimensional model of male chest

The forearm diameter can be 0.033 to $0.04 \mathrm{~m}$ for an adult. The forearm model, shown in Figure 4.7, has been developed from Figure 4.2 by fitting into a circle of $70 \mathrm{~mm}$ diameter. This model includes two concentric circles to mark the skin and outer periphery of muscle tissues. The muscle layer includes the flexor digotorum profunds, flexor digontorum superficial and interosseous membrane. Uniform thermal and physical characteristics will have been chosen for the muscle layer; though constructing multi-type tissues have dissimilar functionalities but almost 
similar thermal behavior. Two bigger circles in the muscle layer indicate the ulna and radius bone tissues and their respective arteries are modeled by other two smaller circles. The bone and artery circles are fitted with their sizes as well as the locations. However narrow arteries and vein were neglected because of their insignificant effects on the skin thermal features. The synopsis of the model is presented in the Table 4.1. Assuming the center of forearm model at $(0,0)$ the location of bones and arteries are determined. For example, the center of the radius bone is located at $5 \mathrm{~mm}$ right and $13 \mathrm{~mm}$ down from the forearm model center. Likewise the location and size of other domains are tabulated in Table 4.1. The irregular shapes of bones are omitted and assumed to have a round shape.

Table 4.1 Synopsis of forearm

\begin{tabular}{|l|c|c|}
\hline Tissue Type & Diameter $(\mathrm{mm})$ & Location $(\mathrm{mm})$ \\
\hline Forearm & 70 & $(0,0)$ \\
\hline Radius & 16.74 & $(5,-13)$ \\
\hline Ulna & 16.74 & $(15,18)$ \\
\hline Radial Artery & 2.57 & $(-12,-18)$ \\
\hline Ulna Artery & 2.57 & $(-10,13)$ \\
\hline
\end{tabular}

Thermal analysis of forearm tissues is limited only on $1 \mathrm{~cm}$ surface, shown in Figure 4.2(a), because the outer radius and the inner structure are not uniform everywhere on the forearm. Therefore $1 \mathrm{~cm}$ portion of forearm near the elbow has been chosen for developing the model. The three dimensional physical model of the forearm is shown in Figure 4.8. The "green" solid rods are the ulna and the radius and the "orange" solid rods are the respective capillaries.

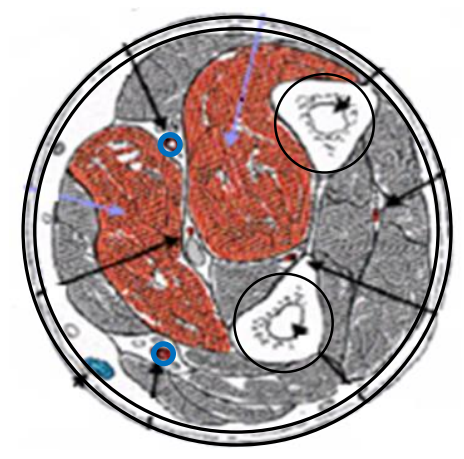

(a)

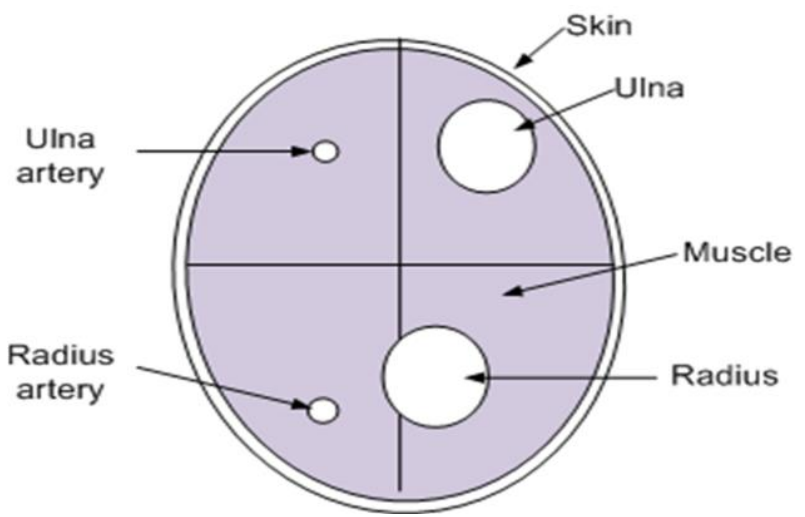

(b)

Figure 4.7 Human forearm diagram-(a) Cross-sectional diagram, and (b) Development of forearm model base 


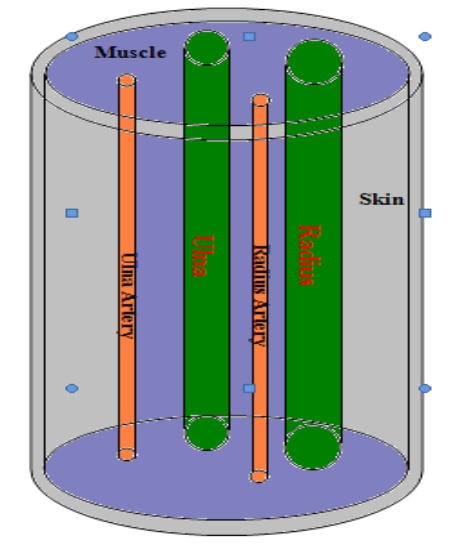

Figure 4.8 Three-dimensional model of forearm

\subsubsection{The Breast (Hemispherical Organ) Model}

The female breast, an organ that prone to cancer, has been drawing an increasing interest to researchers for decades. Several simple models have already been developed $[72,58]$ based on the cross-sectional view which is shown in Figure 4.3. The existing breast models have two major limitations - the sandwiched fat layer between muscle and lobule was not shown up and the deformations of breasts due to body posture and ptosis were not accounted. Therefore the model does work for thermal analysis only for natural breasts at supine on back position. The research has proposed the physical model of natural breasts with considering the fat layer as well as accounting for body posture. In addition the deformity of ptosis breast has also been modeled.

The natural breast has a circular base of diameter $14.4 \mathrm{~cm}$ and the vertex of $72 \mathrm{~mm}$ which can be considered as a hemisphere of radius $72 \mathrm{~mm}$ as shown in Figure 4.9. Breast is founded on $10 \mathrm{~mm}$ thick thoracic wall; the other tissue layers are following - the $7 \mathrm{~mm}$ width muscle layer, the soft tissue layer (lobule) is a hemisphere of radius $59 \mathrm{~mm}$ which is surrounded by a fat layer of thickness $5 \mathrm{~mm}$ and the $3 \mathrm{~mm}$ thin skin layer covering the outer-fat layer. At the apex, the projected part of the breast is called the nipple which is surrounded by areola tissues. Because of the inconsistent structure of the nipple and the milk duct, these tissues are not considered in developing the breast model. Considering the likeness of the thermal and physical features of the thoracic wall and muscle tissues these two layers can be considered a single domain. Three of the above mentioned four domains (excluding the thoracic wall-muscle layer) are marked with dotted lines which are shown on the cross-sectional diagram. The straight line joining the nipple center 
and breast base passes through the muscle-fat interface which is considered as the model center. Two outer circles have been drawn from the model center with radius $72 \mathrm{~mm}$ and $69 \mathrm{~mm}$, respectively. The inner circle enclosing the lobule tissues has center on the fat-lobule interface (bottom) plane and radius $59 \mathrm{~mm}$. In addition, the chest wall tissues and muscles are considered as solid rectangles of overall $17 \mathrm{~mm}$ width. Assuming the lobule tissues are encompassed by a fat layer, which it is clearly learned from the Figure 4.3, the sandwiched fat layer between muscle and lobule was not shown in the previous models $[58,72]$. For an adult female, a non-deformed typical breast has average $72 \mathrm{~cm}$ radius overall where the thoracic wall and muscle are 7 and $10 \mathrm{~mm}$ thick, respectively. The lobule radius is $59 \mathrm{~mm}$ and the fat layer is approximately $5 \mathrm{~mm}$ thick, which was obtained from [72]. Considering the areola region is a curvature of radius $7.2 \mathrm{~cm}$, subtend angle $20.33^{\circ}$ and thickness of $3 \mathrm{~mm}$. The ribs and blood veins are also shown in the model.

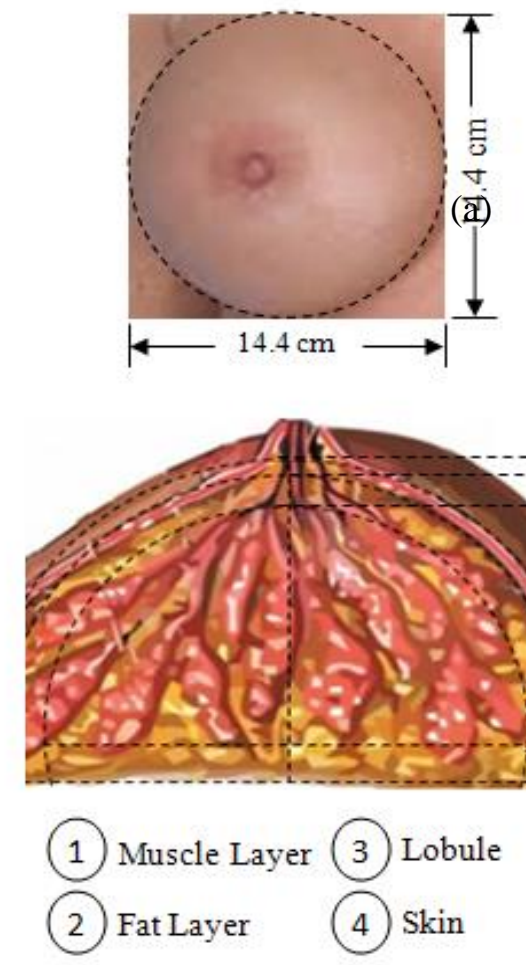

(c)

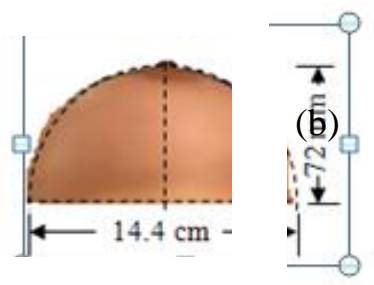

Fig

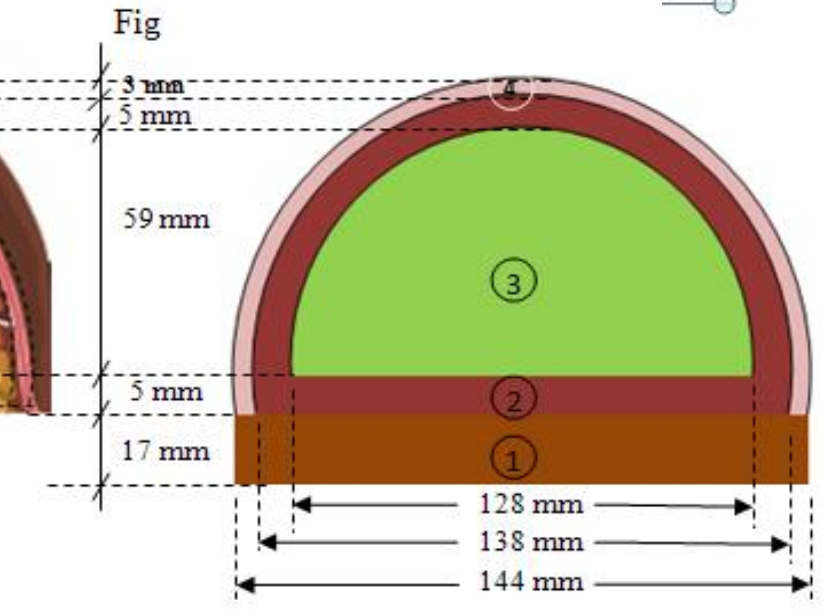

(d)

Figure 4.9 Breast diagram-(a) and (b) Physical dimensions, (c) cross-sectional diagram, and (d) developed model for breast cross-section

The three-dimensional anatomically accurate thermal model of a natural, non-deformed female breast is shown in Figure 4.10. The domains are indicated by different colors- the "red" 
domain is muscle and thoracic wall, the "white" zone is skin, the "blue" layer is the fat and the "light green" indicates the mammary gland tissue layer.

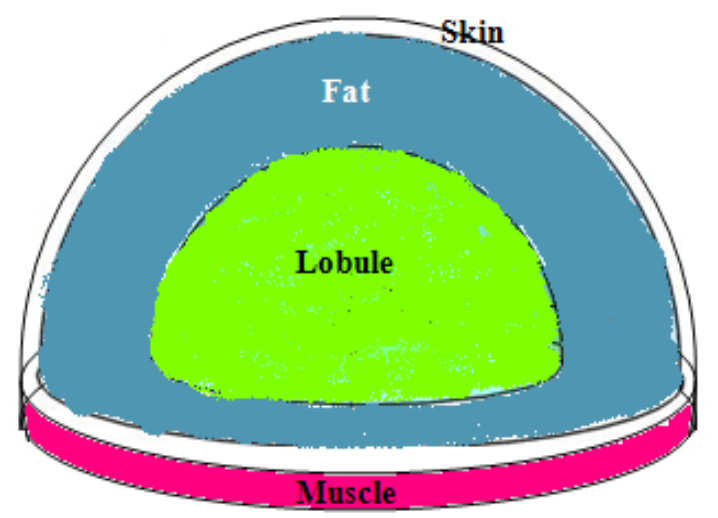

Figure 4.10 Three-dimensional model of breast

Breast tissue is a kind of viscoelastic tissue that shows large deformation. Therefore, conventional model is not useful for computing accurate thermal features of deformed breasts. Although previous thermal-modeling studies did not take into account breast deformations caused by gravity. In this study, a new method has been proposed for modelling the deformed breasts. The deformation of breast tissue due to gravity (the change of imaging body position) is analyzed to reconstruct the model which is a kind of inspection method and is able to analyze mild to severe deformations.

It has been noted, however, that the breast geometry may deform substantially merely because of body posture changes [61], and that the nonlinear elasticity effects associated with large

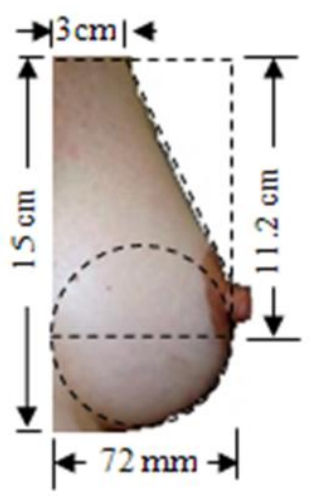

(a)

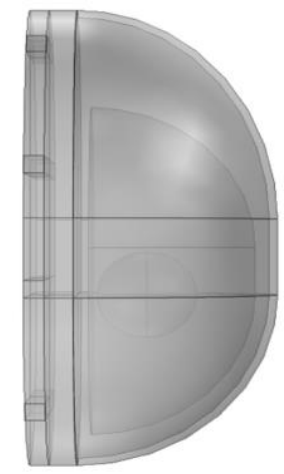

(b)

Figure 4-11 Deformed breast-(a) Mildly deformation due to standing posture and (b) Developed model for mildly deformed breast 
deformations may not be negligible [62]. A recent simulation study using combined thermal and elastic modeling [62] indicated that the gravity-induced deformation alone can affect the temperature distribution throughout the breast. However, to the best of our knowledge, up to now there has still been a shortage of simulations studying the relation between skin thermal expression of deformed breast and interior pathophysiological information, such as tumor, inflammation and infection.

In the standing position the female breast, even the apparently looking natural breast, deforms significantly which can be understood from Figure 4-11. The outlook of deformed breast as shown in Figure 4-11(a) has divided into two parts - the bottom part (below the nipple) still has a hemispherical structure and the upper part (above the nipple) has almost a conical structure. The three-dimension comprehensive thermal model of breast in standing position is developed mimicking the anatomical structure and physiological characteristics, which are shown in Figure 4-11(b). Assuming the deformation is caused by gravitational force only and the force acts vertically downward, therefore, the height of breast will remain unaffected which is $72 \mathrm{~mm}$ (as it was for the natural breast). Taking the mass and tissue density into account the length (top-down) of the breast is calculated to be $15 \mathrm{~cm}$ and the height of the conical part is $11.2 \mathrm{~cm}$.

Permanent deformity of the breast is called ptosis. Ptosis patients are categorized into six different scales, which are discussed in section 1.4 of Chapter 1. For a mild drooping, the nipple is slipped downward from the apex and in many cases it could be noticed only if the patient standing or sitting. The deformation measure of such (Grade-1) ptosis is similar to deformation likely found in natural breast at the standing body posture, as discussed above, and needless to explain again. However, the only exception is the gentle nipple shift of mild ptosis patient causes to build a relatively thicker fat layer at the top part of breast keeping the lobule layer un-deformed. It is due to the lesser elasticity of the adipose layer than the fibroglandular tissues.

The drooping might continue because of aging, pregnancies, malnutrition, excessive physical exercise or any other physiological deformations and downward sliding of the nipple could reach far below the infra-mammary fold and at the lower level of maximum breast projection and could points toward the floor in case of severe ptosis (Figure 4.12a). Unlike mild ptosis, in the case of a severe deformation (for example: Grade-IV) breast nipple slips below the breast base and both the outer and inner structures of breast are altered as shown in Figure 4.12b. The physical 
geometry of the deformed breast has three segments - the hemispherical part (lower part around the nipple), the cylindrical part (middle part) and the cylindrical wedge part (upper part). The cylindrical wedge part produce a subtend angle $45^{\circ}$ with the breast base. Ptosis breast develops a non-uniform fat layer while most of the fat is stored at the top. Unlike mild ptosis, the mammary gland also deforms and which has divided into two parts where the bottom part has hemispherical structure and the upper part has cylindrical structure. The dimensions of each segment have been labeled on the model. The breast base (i.e. the thoracic wall and muscle layer) diameter is $144 \mathrm{~mm}$ and the distance between the lower part of the breast base to the

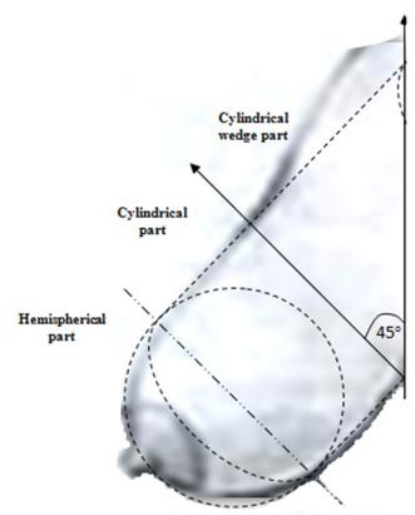

(a) Severe ptosis breast

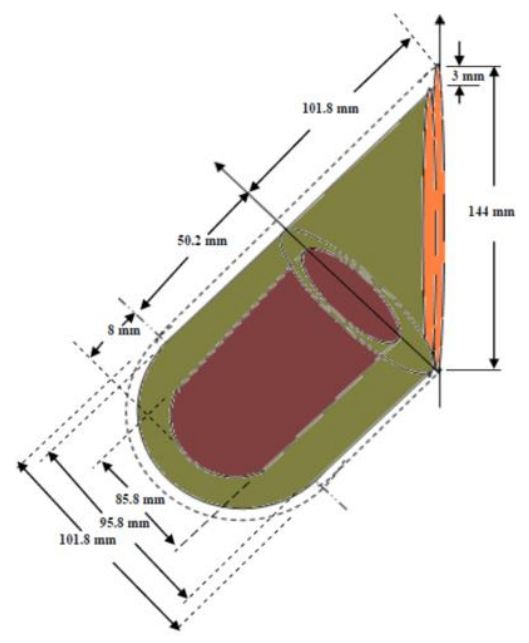

(b) Ptosis breast Model

Figure 4.12 Ptosis breast and model

nipple is $72 \mathrm{~mm}$, which are obtained from the natural breast geometry, will be remain unchanged. However, the length between upper-part of breast base to the nipple will be increased due to the elastic deformation which is measured $168 \mathrm{~mm}$ - the length wedge segment is $101.2 \mathrm{~mm}$, the cylindrical segment is $50.8 \mathrm{~mm}$ and the radius of the hemispherical segment is $50.9 \mathrm{~mm}$.

\subsection{Summary}

Developments of anatomically accurate thermal models are a prerequisite to forecast thermal behavior of an organ. The anatomy of an organ could provide the necessary information about the number of tissue layers, layer thicknesses, heat loss surface area etc. which play a vital role to develop the realistic model. To understand the temperature variation on different regions in the human body the male chest, forearm and female breast have been modeled. 


\section{Chapter 5 Thermal Analysis of External Organs with Embedded Tumor}

\subsection{Introduction}

In the previous chapter, thermal features on benchmark tissue models at healthy condition have been addressed analytically (Equations. 3.10-3.12, 3.27 and 3.46, respective temperature flow

in Figure 3.4, 3.5 and 3.6) and numerically (Figure 3.12) with deliberating general influences of thermal and biological parameters on tissue's interior temperature distributions (Figure 3.15-3.22). The analytical and numerical results were in good agreements (Figure 3.12) for the cases studied in Chapter 13. However, the analytic solutions are obtained on simplistic (homogeneous) models and cannot be applied for clinical application. Since human tissues exhibit behavioural diversities in terms of heat generation and temperature evolution, anatomical accurate models are a must requirement predicting for accurate thermal behaviour. In addition analytic solution cannot be applied to compute abnormal local surface temperature distribution which is an obvious phenomenon in case of an embedded hyperactive nodule. The realistic models have also paved their ground in inverse computing the physical and thermal behaviours of implanted hyperactive regions from the peripheral abnormal thermogram. Development of the pragmatic models of chest, forearm and breast (natural and deformed), by mimicking their anatomical structures, was discussed in Chapter 34. Estimating thermal features of cancerous organs, while implanting a hot nodule inside the proposed models, is the main objective of this chapter. A brief description of the contemporary models of organs with buried tumors is presented in the opening section of the chapter, followed by the author's perception relating to the importance of imitating the anatomical structure accurately in developing physical models. In addition, the distinguishing behaviours of malignant and benign tumors in terms of heat generation and dissipation have also been addressed. Moreover, numerical simulations for computing thermal behaviours of faulty organs using finite element method have been detailed. Finally, the chapter demonstrates that important diagnostic parameters of a tumor are implied in the abnormal thermal behaviour they exhibit, thus invoking an inverse process that may be effectively fit in revealing significant diagnostic (clinical) parameter 
Numerical approach in solving bio-thermal problem involves preparing a structural (physical) model, developing numerical (such FDM, FEM etc.) models and solving bio-thermal equations. Physical models should be developed mimicking an organ's structure and homogeneity. Bio-thermal physics are to be linked appropriately, the governing equation to the domain of study as well as to the associated boundaries in developing the thermal model. The domain of study is, then, to be discretized into small elements which is called the meshing process. Finally thermal equations are solved repeatedly until estimates converge to a desired accuracy. All the processes can be done by well-known numerical methods, namely the FEM, FDM, MOM and others. State of the art approaches in addressing bio-thermal problems are discussed below:

A second order finite difference scheme was implemented for solving the bio-heat Pennes' equation with mixed boundary conditions in 2010 A.D. by Agnelli et al. [14]. The authors proposed a cuboid thermal model consisting of two domains - the healthy tissue and the spherical tumor tissue where the tumor was encompassed by the healthy tissues. The authors estimated the temperature distribution in the tissue interior, as well as on the surface, despite the fact that the modelled organ was unknown, resulting the clinical applications debating. However, as best to the author's knowledge, this was the only work credited for proposing a three-dimensional rectangular thermal model while the other studies were limited to the modeling of the breast only.

The hemispherical structure and the heterogeneity of the breast were modelled comprehensively by Sudershan et al. [58]. The weakness of the model is discussed in Chapter 3. However, in the recent past, almost all previous studies had been using the very same model to estimate the thermal features of a breast tumor. While estimating the surface temperature and taking into account the variations in the metabolic heat generation, blood perfusion and vascular thermal interactions of each tissue layers as well as in the tumor, the authors [60] remarked that there was a direct relation between the breast surface temperature and the embedded tumor beneath the breast. The result contradicted the earlier findings of Osman and Affify [72]. The reason behind the failure of Osman et al. was the poor grid selection (meshing) of domain discretization. Mesh size effect, subject to change with domains sizes, could be optimized by parametric study in conventional approaches, plays a vital role to the accuracy of the output results. However, the commercial numerical solvers have some built-in features to define the optimum element size. Mital et al. [60, 16] used a finite element meshing, using ANSYS commercially available package to solve the Pennes' bio-heat equation in order to find the surface temperature. The Monte Carlo 
method was implemented to solve the transient three-dimensional bio-heat transfer problem with non-linear boundary conditions (simultaneously with convection, radiation and evaporation) and space-dependent thermal physiological parameters [75] and reported that thermal states of biological bodies, reflecting physiological conditions, could be correlated to the temperature or heat flux mapping recorded at the skin surface. In addition to the thermal analysis on natural (undeformed) breasts, it is the only paper [76] where Jiang et al. considered the gravity-induced elastic deformation to develop a 3D finite element method based on thermal and elastic model of breast in various body postures, to characterize comprehensively both the thermal and elastic properties of normal and tumor breast tissues during static and dynamic thermography.

Inspecting the results of the research in $[14,20,60]$ the major lacking in the accurateness of models, the inconsistency in defining the bio-thermal parameters, the contradiction in finding the dependency of temperature texture on bio-thermal parameter. This study has resolved the former drawback by replacing the existing models with realistic models proposed in Section 0; while the latter drawbacks will be solved in the subsequent sections with assigning accurate tissue's thermal behaviours, precise meshing (finite element) and adopting all possible situations in developing the mutual dependency of parameters.

\subsection{Tumor Thermal Behavior}

Understanding thermal behaviour of tumors was a key concern for researchers for centuries. In 1963, it was first investigated by Lawson and Chugtai [13] that every mammary carcinoma is hotter than its artier blood supply and so was the venous drainage from tumor. A later study by Gautherie [15] also confirmed the similar result during the surgical operations on breast cancer patients and remarked that it (getting hotter locally) is a general sign of tissues or organs with high metabolic rates. In connection with the physiopathological viewpoint, it is said that the greater the blood perfusion to a tissue, the greater the local metabolism and the greater the quantity of local heat generation. Therefore the cancerous tissues have much higher blood flow rate than healthy tissues. Consequently, tissue thermal conductivity of cancer tissue is likely to increase than that of healthy tissue. An in-vivo analysis using finite-needle probe discovered that the average thermal conductivity of cancer tissues is $0.511 \mathrm{~W} /\left(\mathrm{m} \cdot{ }^{\circ} \mathrm{C}\right)$ due to the excessive blood supply in the defective cells which can be enhanced by $0.031 \mathrm{~W} /\left(m .{ }^{\circ} \mathrm{C}\right)$ compared with that of surrounding healthy gland tissues [68]. In a quantitative study [77], L. Priebe et al. estimated a correlation 
between the change in volumetric blood flow rate and the thermal conductivity. The authors proved experimentally (in-vitro mode) that an increase in blood flow of $150 \mathrm{ml} / 100 \mathrm{~g} / \mathrm{min}$ (150 millilitres of blood per 100 grams of tissues per minute) causes an enhancement in thermal conductivity of $0.05 \mathrm{~W} /\left(m{ }^{o} \mathrm{C}\right)$. Therefore, a solid correlation between the change in thermal conductivity $(\nabla k)$ and the blood perfusion rate $\left(\omega_{b}\right)$ can be achieved as:

$$
\omega_{b}\left[L /\left(g m-{ }^{o} C\right]=30 \nabla k\left[W /\left(m-{ }^{o} C\right)\right]\right.
$$

From above investigations, it is evident that cancer may be considered as a tissue on account of higher metabolic and blood perfusion rate and also a higher conductivity compared with surrounding tissues. However, determination of the metabolic heat generation of cancer tissue in terms of quantifying it is certainly complex because of the extreme intricacy of the biochemical reaction involved as well as their variability from one type of cancer to the other. Gautherie [15] performed an experiment on 84 patients with relatively small cancer (tumor size between 9 and 38 $\mathrm{mm}$ ) and found that the tumor metabolic heat generation is related to the doubling time by nearly hyperbolic law, whereby the faster the tumor grows the more heat it produces. In brief, the relation between them can be expressed as follows:

$$
Q_{m} \cdot \tau=3.27 \times 10^{6} \quad\left(\mathrm{~W} . \text { day. } \mathrm{m}^{-3}\right)
$$

where $\tau$ is the time needed for the tumor to double its volume, $Q_{m}$ is the volumetric heat generation rate. Finally, the relation between the tumor size $(d)$ and $\tau$ was found as:

$$
d=10 e^{2.134 \times 10^{-3}(\tau-50)}
$$

Therefore, the tumor thermo-physical parameters such as the thermal conductivity, perfusion rate, heat generation rate, and diameter are interdependent as the empirical way presented in Eqns. (5.1) through (5.3), which display influential effect on thermal features.

\subsection{Tumor Shapes}

An abnormal growth of tissues creates lumps or tumors which perform no useful body function and grow at the expense of healthy tissues. A cancerous tumor is a mass of tissue that is growing in an abnormal, uncontrolled way. It may invade surrounding tissue, or shed cells into the

bloodstream or lymph system. The relatively denser mass appears whiter than any tissue around it while doing biopsy. Biopsy also discovers tumor shapes. Tumors have a wide range of shapes, 
sizes and contrast, in particular, a breast tumor is very hard, like a bit of raw carrot. They will also have an irregular shape, and feel bumpy (not smooth). Foretelling about tumor shape without surgery is still trivial. However, this study will detail a novel methodology to project tumor shape from thermogram.

Depending on the physio-thermo-biological natures, tumors can be divided broadly into two categories - benign and malignant. Generally benign masses are well circumscribed and compact, such tumors possess well-defined shape and sharp boundaries that delineate them from surrounding tissues. Inspections explore that most of this type usually are round or oval in shape, but some cases may be partially round, with a spiked or irregular outline as part of their circumference. Masses having irregular shapes and spiculated or indistinct margins suggest a higher possibility of malignancy. A multi-pointed star-shaped outline mass is described as spiculated. Figure 5- 1 presents different shapes and margins of breast tumors likely observed in biopsy or anticipated by computer techniques.

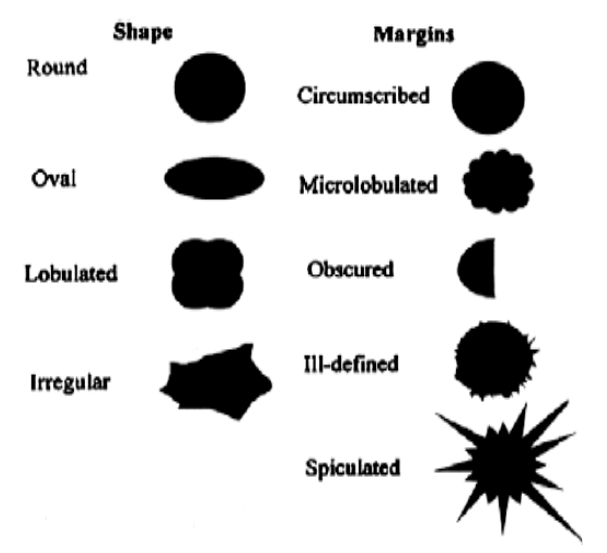

Figure 5- 1 Tumor shapes and margins

Computer techniques proposed to date for tumor analysis have concentrated mainly on shape and texture measures. Shape measure based on isothermal contours provides some useful indication about the shape complexities that might be observed between circumscribed and spiculated tumors; however, they are not designed to characterize the density variations across the boundary of a tumor.

The uncertainty in shapes and margins may have remarkable impact on thermal features and needs to be investigated properly. However, the analysis in this chapter is limited to the spherical tumors only in order to avoid the complexity of relating unique shapes with thermal features. 


\subsection{Thermal Parameters}

Tissues are groups of similar cells that perform a common function. There are four categories of tissues in the human body: epithelial, connective, nervous, and muscle. Epithelial tissue (covering and lining epithelium, and Glandular epithelium) protects the human body from moisture loss, bacteria, and internal injury. Connective tissue (loose connective and fibrous connective tissues) generally provides structure and support to the body. Nervous tissue forms the nervous system, which is responsible for coordinating the activities and movements of the body through its network of nerves. Muscle tissue differs from other tissue types in that it contracts. Muscle tissue comes in three types: cardiac, smooth, and skeletal. Those muscle tissues are made up of muscle fibers. The muscle fibers contain many myofibrils, which are the parts of the fiber that actually contract. Not only are the purposes and functions different as discussed, but the tissues differ from one another in terms of their biological and thermal behavior. Characteristic parameters controlling the thermal features in each tissue layer were first introduced in the landmark paper by Pennes, which appeared in 1948. In that study, the author discovered the governing equation for the bio-thermal model of a living organ (see Eqn. 4.1). Applying on the Pennes' bio-heat transfer model, numerous studies, since then, have disclosed that thermal and biological properties of tissues play a critical role in the study of tumour detection and localization. Parameters such as heat conductivity, density, specificity, and metabolism incur some significant attributes to temperature images. Arguably, previous research shows inconsistency in evaluating these parameters and consequently leads to unconvincing findings. Certainly, the accuracy of the thermal analysis requires, first and foremost, the appropriate choice of the biological and thermal parameters of the healthy tissue layers as well as the tumour. Before sorting the exact values out, the suggestive bio-thermal values of pioneer researcher are briefed in the following: 
Table 5- 1 Thermal parameter of blood (assumed in previous studies)

\begin{tabular}{|c|c|c|c|c|c|c|c|c|c|}
\hline \multirow{2}{*}{$\begin{array}{l}\frac{7}{0} \\
0 \\
0 \\
0 \\
0 \\
0 \\
0\end{array}$} & \multirow{2}{*}{$\begin{array}{c}\begin{array}{c}\text { Blood } \\
\text { perfusion rate, }\end{array} \\
\omega_{b} \\
\left(\frac{1}{s}\right)\end{array}$} & \multicolumn{2}{|c|}{$\begin{array}{c}\text { Blood } \\
\text { Density, } \rho_{b}\end{array}$} & \multicolumn{2}{|c|}{$\begin{array}{c}\text { Blood specific } \\
\text { heat, } c_{b}\end{array}$} & \multicolumn{2}{|c|}{$\rho_{b} \omega_{b}$} & \multirow{2}{*}{$\frac{c_{b} \rho_{b} \omega_{b}}{\frac{W}{m^{3} K}}$} & \multirow{2}{*}{$\begin{array}{l}\text { Tissue } \\
\text { type }\end{array}$} \\
\hline & & $\frac{g m}{m l}$ & $\frac{\mathrm{kg}}{\mathrm{m}^{3}}$ & $\frac{\mathrm{Cal}}{\mathrm{gm^{ \circ } \mathrm { C }}}$ & $\frac{J}{k g K}$ & $\frac{g m}{m l} \frac{1}{s}$ & $\frac{\mathrm{kg}}{\mathrm{m}^{3}} \frac{1}{\mathrm{~s}}$ & & \\
\hline [78] & & & 1000 & & & $\begin{array}{r}0.00025 \\
0.0005\end{array}$ & $0.25-0.5$ & & \multirow{8}{*}{$\begin{array}{l}\text { Muscle, } \\
\text { gland }\end{array}$} \\
\hline [67] & $\begin{array}{c}0.0002 \\
0.00025 \\
0.0003\end{array}$ & 1 & 1000 & 1 & 4186 & $\begin{array}{l}0.00020 \\
0.00025 \\
0.00030\end{array}$ & $\begin{array}{c}0.2 \\
0.25 \\
0.3\end{array}$ & $\begin{array}{c}837.2 \\
1046.5 \\
1255.8\end{array}$ & \\
\hline [68] & 0.0003 & 1 & 1000 & 1 & 4186 & 0.00030 & 0.30 & 1255.8 & \\
\hline [52] & & & & 0.919 & 3850 & & 0.3 & 1155 & \\
\hline [51] & 0.00052 & 1 & 1000 & 1 & 4186 & & 0.52 & 2176.72 & \\
\hline [79] & 0.0005 & & 1000 & 0.86 & 3600 & & 0.5 & 1800 & \\
\hline $\begin{array}{c}{[58,59,} \\
53]\end{array}$ & 0.00054 & & 1060 & 1.003 & 4200 & & 0.57 & 2400 & \\
\hline [14] & 0.000477 & & 1000 & & 4186 & & 0.477 & 1998.1 & \\
\hline $\begin{array}{c}{[58,59,} \\
53]\end{array}$ & 0.00018 & & 1060 & 1.003 & 4200 & & 0.19 & 800 & $\begin{array}{c}\text { Areola } \\
\text { Fat, } \\
\text { skin }\end{array}$ \\
\hline
\end{tabular}

\subsubsection{Blood Thermal Parameters: Density $\left(\rho_{b}\right)$, Specific Heat $\left(c_{b}\right)$ and Perfusion Rate $\left(\omega_{b}\right)$}

Heat generated due to metabolic activity is partly lost through perfusion, which depends on the perfusion rate, blood density, and blood specific heat (see Eqn. 4.1). Authors in [51, 14] had recommended blood density $1000\left[\frac{\mathrm{kg}}{\mathrm{m}^{3}}\right]$ whereas $1060\left[\frac{\mathrm{kg}}{\mathrm{m}^{3}}\right]$ was considered in $[23,25,19]$. The specific heat, the amount of heat required to increase per kg blood by one degree Kelvin, was

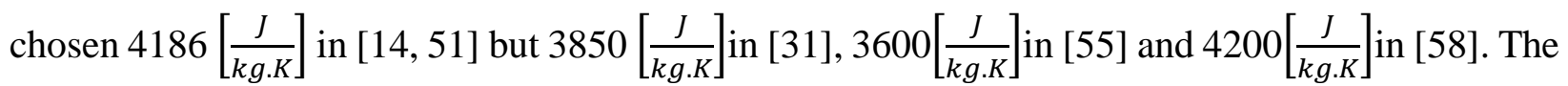
volumetric blood flow per second in muscle or gland tissues was considered between $2 \times 10^{-4}$ and $5 \times 10^{-4}\left[\frac{\mathrm{gm}}{\mathrm{ml.S}}\right]$ in $[67,72]$ while Minhua et al [51] applied $0.52\left[\frac{\mathrm{kg}}{\mathrm{m}^{3} . \mathrm{S}}\right] . \mathrm{Ng}$, a pioneer researcher in this field used heat loss amount due to blood perfusion $2400\left[\frac{W}{m^{3} . K}\right]$ in $[22,23]$ and the same value was also recommended in [55]. As the blood perfusion rates differ in different tissue layers, the previous papers had introduced the differences and assigned one-third of muscle-gland (lobule) layer perfusion loss to areola-fat-skin layers. In the most recent work [14] assumed this value $1998.1\left[\frac{\mathrm{W}}{\mathrm{m}^{3} \cdot \mathrm{K}}\right]$. The thermal parameters of blood used in previous research are presented in Table 5- 1, where the boldface values with respective units are found directly on their papers. Other 
values are computed from that (the boldface values) by converting the units in order to make an easily understandable comparison. It is seen that blood perfusion $\rho_{b} \omega_{b}$ for muscle/gland tissues was widely chosen between 0.2 and $0.52\left(\frac{\mathrm{kg}}{\mathrm{m}^{3}}\right)\left(\frac{1}{\mathrm{~s}}\right)$, however, the relatively reliable studies limit their assumption to between 0.477 and $0.52\left(\frac{\mathrm{kg}}{\mathrm{m}^{3}}\right)\left(\frac{1}{\mathrm{~s}}\right)$.

Table 5- 2 Conductive thermal coefficient

\begin{tabular}{|c|c|c|c|}
\hline \multirow[t]{2}{*}{ Reference } & \multicolumn{2}{|c|}{ Thermal Conductivity, $\mathrm{k}$} & \multirow[t]{2}{*}{ Tissue Type } \\
\hline & {$\left[\frac{\mathrm{cal}}{\mathrm{s.cm} \cdot{ }^{\circ} \mathrm{C}}\right]$} & {$\left[\frac{W}{m \cdot K}\right]$} & \\
\hline [2] & 0.0005 & 0.21 & \multirow[t]{5}{*}{ Muscle } \\
\hline [3] & 0.00015 & 0.627 & \\
\hline$[4][8,9][11]$ & & 0.48 & \\
\hline [10] & & 0.51 & \\
\hline [5-7] & & 0.5 & \\
\hline$[9,10]$ & & 0.21 & Areola \\
\hline [9] & & 0.21 & \multirow[t]{2}{*}{ Fat } \\
\hline$[10]$ & & 0.22 & \\
\hline$[9,10]$ & & 0.48 & Gland \\
\hline$[10]$ & & 0.27 & Skin \\
\hline
\end{tabular}

\subsubsection{Thermal Parameters of tissues: Thermal Conductivity, Density and Specific Heat}

Tissues' thermal feature is controlled by conduction heat transfer rates, densities and specific heats. Deducting the perfusion heat loss, the remaining metabolic heat is transferred toward the surface by the means of conduction. Pennes' [67] preferred the tissue thermal conductivity $k=$ $0.0005[\mathrm{cal} /(\mathrm{s.cm} . \mathrm{K})]$, however, later his assumption was proven roughly one-third of the realistic value [68]. Recently, the average heat transfer rate of muscle tissues are accepted between 0.48 and $0.51[\mathrm{~W} /(\mathrm{m} . \mathrm{K})][14,57,72]$ and between 0.21 and $0.22[\mathrm{~W} /(\mathrm{m} . \mathrm{K})]$ for areola and fat tissues $[25,60]$. The density and specific heat of tissues play a significant role in the dynamic thermal analysis, however, have no effect to the thermal analysis in the steady state, and therefore are skipped in this study. Thermal conductivity values recommended in previous studies are listed in Table 5- 2. It is observed that muscle layer conducts heat more profusely than any other tissues.

\subsubsection{Metabolic Heat Generation}

Metabolism is the live phenomena that produces heat and keeps the body warm. Study [56, $67,51]$ assumed the metabolic heat generation rate $Q_{m}=418.6\left[\mathrm{~W} / \mathrm{m}^{3}\right]$. For breast soft tissues the value is $700\left[\mathrm{~W} / \mathrm{m}^{3}\right]$ and for fat, skin, lobule and areola tissues is $400\left[\mathrm{~W} / \mathrm{m}^{3}\right]$ according [59, 
16]. The works reported solving the bio-heat problems analytically, where $[69,51]$ assumed the rectangular geometry while works $[68,52]$ dedicated for the cylindrical living tissues used heat generation rate $1085\left[\mathrm{~W} / \mathrm{m}^{3}\right]$. But surprisingly study reported in [14] applied $4200\left[\mathrm{~W} / \mathrm{m}^{3}\right]$ which was not supported by any other sources (it took roughly 6-10 times higher than other woks). Table 5- 3 lists the suggested values of metabolic heat rate for different tissues at healthy condition. It is seen, though, that several studies applied $418.6 \mathrm{~W} / \mathrm{m}^{3}$, but the most recent works have taken around $700 \mathrm{~W} / \mathrm{m}^{3}$.

Table 5- 3 Metabolic heat generation rates

\begin{tabular}{|c|c|c|c|}
\hline \multirow{2}{*}{ Reference } & \multicolumn{2}{|c|}{ Metabolic heat, $Q_{m}$} & \multirow{2}{*}{ Tissue Type } \\
\cline { 2 - 3 } & $\frac{\text { Cal }}{\mathrm{sm}^{3}}$ & $\frac{W}{m^{3}}$ & \\
\hline$[68,67]$ & $\mathbf{0 . 0 0 0 1}$ & 418.6 & Muscle \\
\hline$[52]$ & & 1085 & \\
\hline$[60,72,58]$ & & 700 & \\
\hline$[14]$ & & 4200 & Areola, Fat, Skin, Gland \\
\hline$[60,72,58]$ & & 400 & \multicolumn{2}{c}{} \\
\hline
\end{tabular}

\subsubsection{Heat Losses Rates at Boundary}

Thermal analysis requires solving the heat transfer problem in different medium; the conditions that the field must satisfy at the interface separating the media are called boundary conditions. The boundary interfaces are: body core-muscle interface, muscle-blood vessel interface, skin-ambient interface etc.; the boundary conditions are presented in Eqn. 4.2. The conditions state that the artier blood or body core temperature, the ambient temperature and the heat exchange coefficients have inclusive influences on heat flow graphs. Referring to Eqn. 4.1, the artier blood (body core) temperature was assumed $310.15 \mathrm{~K}\left(37^{\circ} \mathrm{C}\right)$ by most of the previous works, which was remaining unaffected during the analyses. Recalling the boundaries, the body core is thermally insulated and the heat is entirely exchanged at the skin surface (Eqn. 4.2), the environment temperature was assumed between $25^{\circ} \mathrm{C}$ and $28^{\circ} \mathrm{C}$ and the heat exchanges between skin and environment occur mainly due to convection and radiation process. In the earliest work by Pennes, assumed the total heat loss rate over forearm skin $10^{-4}\left[\frac{\mathrm{cal}}{\mathrm{s.cm}^{2} \mathrm{C}}\right]$ but recent studies reveal that Pennes' [67] assumption was too low otherwise building that kind laboratory environment would not be realistic. Wissler [68], therefore, recommended double of the Penne's assumption. 
Authors in $[10,60]$ solved the problem with taking heat losses factor around $10\left[\frac{\mathrm{W}}{\mathrm{m}^{2} \mathrm{~K}}\right]$ and in $[52]$ addressed the value $13.5\left[\frac{W}{m^{2} K}\right]$.

Table 5- 4 Heat exchange rates

\begin{tabular}{|c|c|c|}
\hline \multirow{2}{*}{ Reference } & \multicolumn{2}{|c|}{ Heat Transfer coefficient, $h_{a}$} \\
\cline { 2 - 3 } & $\frac{c a l}{s . m^{2}{ }^{o} C}$. & $\frac{W}{m^{2} K}$ \\
\hline$[67]$ & $\mathbf{0 . 0 0 0 1}$ & 4.18 \\
\hline$[68,51]$ & $\mathbf{0 . 0 0 0 2}$ & 8.37 \\
\hline$[52]$ & & $\mathbf{1 0 . 0 2 3}$ \\
\hline$[14]$ & & $\mathbf{1 0}$ \\
\hline$[59,56]$ & & $\mathbf{1 3 . 5}$ \\
\hline
\end{tabular}

The diverse choices recommended for the heat exchange rates are summarized in Table 5- 4 while the assumed artery (or body core) and environment temperatures are presented in Table 55.

Table 5- 5 Ambient temperature

\begin{tabular}{|c|c|c|c|}
\hline \multirow[t]{2}{*}{ Reference } & \multicolumn{2}{|c|}{ Temperature } & \\
\hline & ${ }^{\circ} \mathrm{C}$ & $\mathrm{K}$ & \\
\hline [67] & 36.15 & 309.3 & \multirow{4}{*}{ Artier blood $\left(\mathrm{T}_{\mathrm{a}}\right)$} \\
\hline [68] & 36.8 & 309.95 & \\
\hline [52] & 36.85 & 310 & \\
\hline [14] & 37 & 310.15 & \\
\hline$[56,60]$ & 26.6 & 299.75 & \multirow{4}{*}{ Ambient $\left(\mathrm{T}_{\mathrm{e}}\right)$} \\
\hline$[59,60]$ & 24.85 & 298 & \\
\hline [51] & 25 & 298.15 & \\
\hline [79] & 28 & 301.15 & \\
\hline
\end{tabular}

\subsubsection{Thermal Parameter of Tumor}

In general, tumours behave differently from healthy tissues in terms of heat generation and dissipation. Several previous papers suggested that the tissues in hyperactive nodule exhibit remarkably higher metabolic and blood perfusion rate and slightly higher thermal conductivity. More specifically, Agnelli JP et al. [14] assumed $k=0.75$ [W/(m.K)], $\alpha_{b}=c_{b} \rho_{b} \omega_{b}=7992.4$ $\left[\mathrm{W} /\left(\mathrm{m}^{3} . \mathrm{K}\right)\right]$ and $Q_{m}=42000\left[\mathrm{~W} / \mathrm{m}^{3}\right]$ though the authors did not explain the reasonability or the sources of their assumptions. According to their assumptions, the tumour tissues have roughly four times higher perfusion rate, and ten times higher metabolism than the sound tissues. Similarly, studies [70,58] suggested 20 times higher perfusion and 8 times higher heat rate while conduction 
heat flow rate kept unchanged. Moreover, [23, 72, 20, 16] applied $\rho_{b} \omega_{b}=17.2 \frac{\mathrm{kg}}{\mathrm{m}^{3}} \frac{1}{\mathrm{~s}}$ and $Q_{m}$ between $5 \mathrm{~kW} / \mathrm{m}^{2}$ and $65.4 \mathrm{~kW} / \mathrm{m}^{2}$. The list of the recommended tumor thermal parameters on the earlier works can be found in Table 5- 6 .

Table 5- 6 Tumor thermal parameter

\begin{tabular}{|c|c|c|c|c|c|c|c|}
\hline$\ddot{0}$ & $\omega_{b}$ & $c_{b}$ & \multicolumn{2}{|c|}{$\rho_{b} \omega_{b}$} & $c_{b} \rho_{b} \omega_{b}$ & $k$ & $Q_{m}$ \\
\hline $\begin{array}{l}\vec{Q} \\
\overrightarrow{8} \\
\overrightarrow{8}\end{array}$ & $\left(\frac{1}{s}\right)$ & $\frac{\mathrm{kg}}{\mathrm{m}^{3}}$ & $\frac{J}{k g K}$ & $\frac{\mathrm{kg}}{\mathrm{m}^{3}} \frac{1}{\mathrm{~s}}$ & $\frac{W}{m^{3} K}$ & {$\left[\frac{W}{m \cdot K}\right]$} & $\frac{W}{m^{3}}$ \\
\hline$[8,9]$ & 0.01077 & 1060 & 4200 & 11.42 & 48000 & 0.48 & 5500 (Tumour $32 \mathrm{~mm})$ \\
\hline $\begin{array}{l}{[10,} \\
11]\end{array}$ & $\begin{array}{c}0.01622 \\
6\end{array}$ & 1060 & 4200 & 17.2 & 72240 & 0.511 & $\begin{array}{c}65400(\text { dbl in } 50 \\
\text { days }) \\
5000 \text { (dbl in } 650 \\
\text { days })\end{array}$ \\
\hline [7] & 0.00189 & 1000 & 4186 & 1.89 & 7992.4 & 0.75 & 42000 \\
\hline
\end{tabular}

\subsubsection{Recommended thermal parameters}

Reviewing the above mentioned researches, the thermal and biological parameters proposed in this study are presented in Table 5-7. For healthy tissues, muscle and lobule assumed to have same conductive heat transfer and blood perfusion rate, respectively. Fat, areola and skin tissues also have identical thermal conductive coefficient and identical perfusion loss rate. The metabolic heat generation rate is assumed following - the chest muscle produces $420 \mathrm{~W} / \mathrm{m}^{3}$ which is same also for the fat, skin and areola tissues, the breast and forearm muscle tissues produce $700 \mathrm{~W} / \mathrm{m}^{3}$.

Past studies, though, suggested higher thermal values for tumors than healthy tissues but did not explain the reasons, however, the study [77] discovered the hyperbolic relation (Eqn. (5.2)) between the metabolic heat generation and tumor doubling time. After performing in-situ mode experiment the study also proved that increased blood perfusion had caused an enhancement in heat conduction rate which was also supported in [15] and also added that an increase in blood flow of $150 \mathrm{ml} / 100 \mathrm{~g} / \mathrm{min}$ caused an increase in thermal conductivity by $0.05[\mathrm{~W} /(\mathrm{m}$. K)]. Following up the above studies, the mutual dependency of thermal and biological parameters of tumour tissues and their relation with tumour age and doubling time a ranges of values for $k, \omega_{b}$ and $Q_{m}$ (as presented in Table 5-7) are assumed. 
Table 5-7 Thermal and biological parameters

\begin{tabular}{|c|c|c|}
\hline Parameter & Value and Unit & Tissue type \\
\hline \multirow{3}{*}{$k$} & $0.52 \mathrm{~W} /(\mathrm{m} . \mathrm{K})$ & Muscle, Lobule \\
\hline & $0.27 \mathrm{~W} /(\mathrm{m} . \mathrm{K})$ & Fat, Areola, Skin \\
\hline & 0.52 to $0.82 \mathrm{~W} /(\mathrm{m} . \mathrm{K})$ & Tumour \\
\hline \multirow{3}{*}{$\omega_{b}$} & $5.2 \times 10^{-4} 1 / \mathrm{s}$ & Muscle, Lobule \\
\hline & $1.6 \times 10^{-4}$ to $2.2 \times 10^{-4} 1 / \mathrm{s}$ & Fat, Areola, Skin \\
\hline & $1.6 \times 10^{-2}$ to $4.7 \times 10^{-3} 1 / \mathrm{s}$ & Tumour \\
\hline \multirow{3}{*}{$Q_{m}$} & $420 \mathrm{~W} / \mathrm{m}^{3}$ & Muscle (chest, fat, skin, areola) \\
\hline & $700 \mathrm{~W} / \mathrm{m}^{3}$ & Muscle (breast, forearm) \\
\hline & 25000 to $90000 \mathrm{~W} / \mathrm{m}^{3}$ & Tumour \\
\hline$c_{b}$ & $4186 \mathrm{~J} / \mathrm{m}^{3} . \mathrm{K}$ & Everywhere \\
\hline$\rho_{b}$ & $1060 \mathrm{~kg} / \mathrm{m}^{3}$ & Everywhere \\
\hline
\end{tabular}

\subsection{Thermal Analyzer: COMSOL Multiphysics}

The main goal of the study is estimating the thermal feature of the proposed models whilst assigning values to the bio-thermal parameters listed. Recall the analytical formulations for thermal estimation on homogenous tissues (presented in Section 3.3) can't be extended further for the models proposed in Section 4.3. Because the proposed anatomic-accurate physical models Figure 4.6 to Figure 4.10are non-homogenous, impel the analytical approaches out, the numerical methods are proven quite reasonable. Unlike the mathematical formulations in analytical approaches, the numerical methods require discretization (meshing), and repetitive convergence testing. In the previous chapter the consistency of numerical method has been validated by comparing it to the analytical results and demonstrated that numerical methods could be a standalone approach in addressing thermal problems in biological organs. Therefore, the rest of the thermal analyses will be performed numerically in this thesis. Designing domain geometry, linking the domain with bio-thermal governing equations, meshing and convergence testing can be handled efficiently by 'COMSOL Multiphysics' [71] commercial finite element software. This software is used in solving diverse engineering and scientific problems numerically. This software comes with a built-in bio-thermal heat transfer module. Therefore, the superficial and interior thermal behaviour of an organ with hyperactive living tissues can be analyzed numerically more efficiently than 'conventional finite element software'.

However, prior to applying the finite element approach on realistic models, a simplified cuboid tissue model was developed using both finite element approach and Finite Difference 
Method (FDM, traditional numerical scripts codes developed in MATLAB). The results obtained from the developed model using both approaches allowed a validation the FEM analysis.

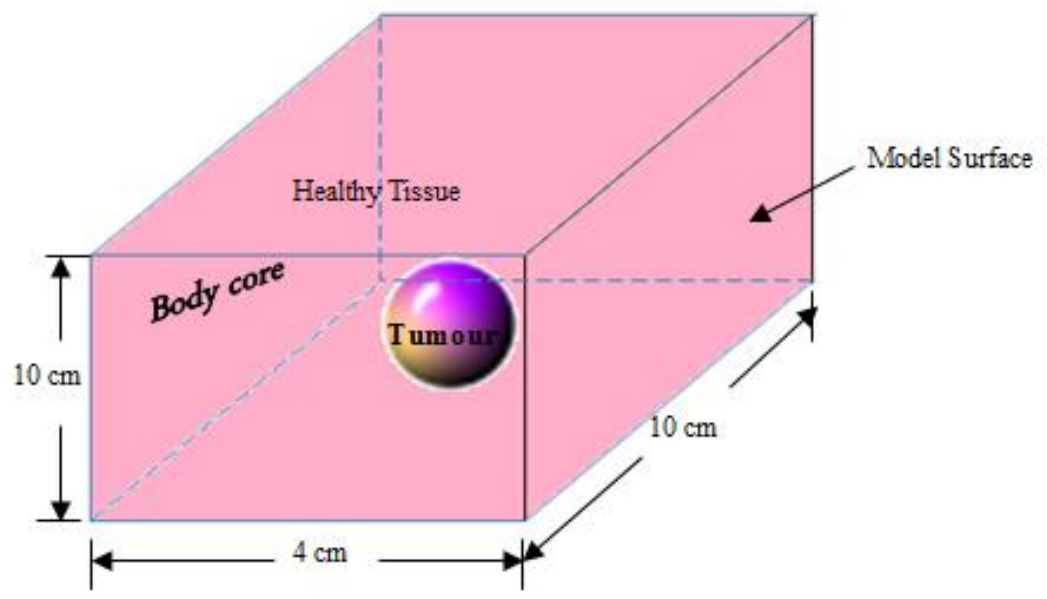

Figure 5-2 Cuboid tissue model 3D (not to scale)

Solving the thermal problem of a cuboid homogeneous tissue domain embedding a 'round' shape, 'circumscribed' margin tumour with conventional FDM approach and the FEM analyzer have been applied on the physical domain which has modelled as a $3 \mathrm{D}$ rectangle implanted with a spherical tumour as shown in Figure 5-2 with delimited dimension $4 \times 10 \times 10 \mathrm{~cm}$ i.e. surface area $100 \mathrm{~cm}^{2}$ and model depth (tissue thickness) $4 \mathrm{~cm}$. The core is assumed to have a constant temperature $310.15 \mathrm{~K}$ (artier temperature) and heat losses due to convection and radiation occurs at model surface and all other lateral boundaries are assumed thermally insulated. The physical model has two sub-domain - the tumour (spherical) and the healthy tissues (cuboid with a spherical hole inside). After some inspection, it was observed that the largest possible tumour (radius $2 \mathrm{~cm}$ ) can affect subtle change in local temperature within $5 \times 5 \mathrm{~cm}$ surface only. Furthermore a larger model domain requires more memory and computation time. Therefore, the $10 \times 10 \mathrm{~cm}$ square surface has been assumed.

\subsubsection{Numeric Models (Gridding and Meshing)}

The domain in Figure 5-2 first discretize into grids for solving the problem using FDM. This is usually done by dividing the domain into a uniform grid (see Figure 5-3). Note that the finitedifference methods produce sets of discrete numerical approximations to the derivative, often in a "time-stepping" manner. Assuming grid spacing factor $h=1 \mathrm{~mm}$, the number of grids (nodes) in $x$ (direction of heat flow) axis is $m=40$ and in $y$, and $z$ axes is $n=100$. The tumour region is 
also divided into nodes. For a $13 \mathrm{~mm}$ diameter spherical tumor, there is 13 nodes at the center and successively decreased by two on the way to the tumour surface. The approximated finite difference model of the tumor is shown in Figure 5-5. The shaded boxes indicate the nodes on the center plane of the tumour. Obviously the number of nodes, and consequently, the accuracy of FDM method depend on the spacing factor $h$. If $h$ decreases the accuracy increase resulting increase in computational memory and time. The dilemma is resolved by the 'trial and error' method and finally come up with $h=1 \mathrm{~mm}$.

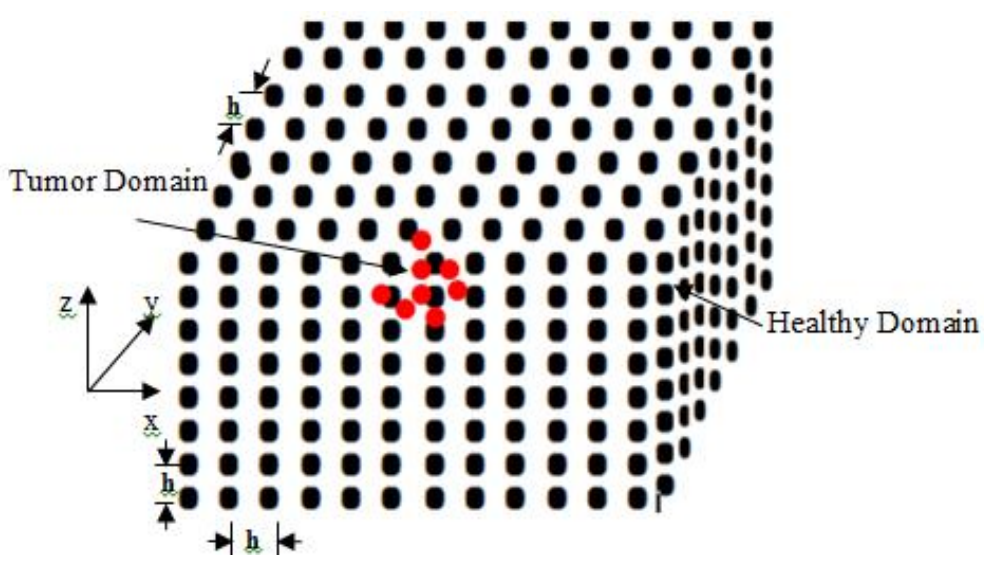

Figure 5-3 Finite difference grids

Alternatively the physical model in Figure 5-2 is divided into small elements while solving with the FEM tools which is called meshing (see Figure 5-4). The meshes are usually tetrahedral inside the domain but triangular around the boundaries as well as around the tumor-healthy domain interface. Applying a custom setting for element size of $1 \mathrm{~mm}$, the analyzer generates 1000 tetrahedral and 500 triangular elements for the overall domain and 10 tetrahedral and 20 triangular elements for the tumor. Moreover the elements are translational in all directions. 


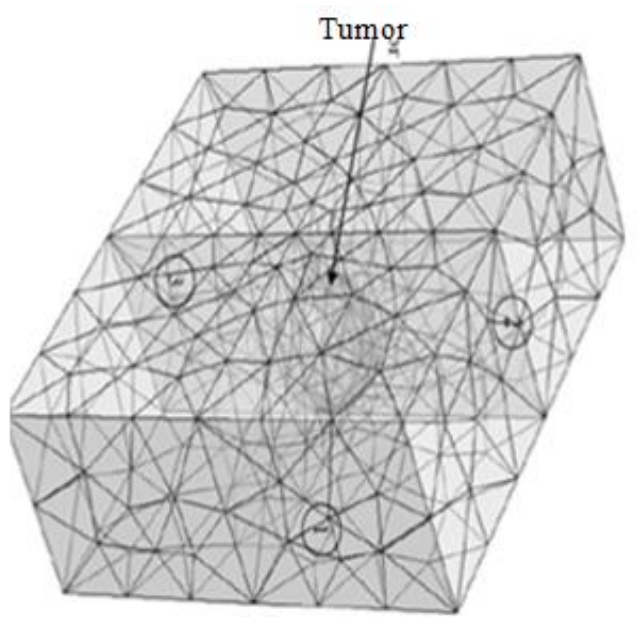

Figure 5-4 FEM mesh diagram

\subsubsection{Coding and Simulation}

Mathematical formulation for thermal analysis in FDM on homogeneous (single) model of healthy tissues was discussed in Section Chapter 1. However, tumor thermal behaviours are significantly different from sound tissue that inquires considering heterogeneity in solving the biothermal problems. Consequently, the terms $Q_{m}, \omega_{b}$, and $k$ require to replace by $Q_{m x, y, z}, \omega_{b x, y, z}$, and $k_{x, y, z}$, respectively, thus that they would change in between domains. Accordingly, the variable $\beta$ and $\forall$ have to replace by $\beta_{x, y, z}$ and $\forall_{x, y, z}$. Modifying Eqns. 3.51 to 3.55 with the above variables and initializing their respective values, the study tailors a MATLAB script for solving the above problem using FDM approach with assuming the tumour centered at the midway of the model i.e. at $(0.02,0.05,0.05)$. In $2 \mathrm{D}$ analysis, the shaded region (lies on center-cut plane passing at $\mathrm{z}=0.05$ ) as shown in Figure 5-5 is assigned with tumor's bio-thermal values while the remaining region holds healthy tissues bio-thermal features. In addition, the all lateral boundaries are set accordingly up, more specifically, the bottom boundary maintains artier temperature, the top boundary radiates and exchange heat with environment and the side boundaries are thermally insulated. Finally, the script is repeatedly computed until any of the stopping criteria meets: a convergence accuracy $\left(\varepsilon \leq 10^{-5}\right)$ or maximum iteration $(N=1000)$. 


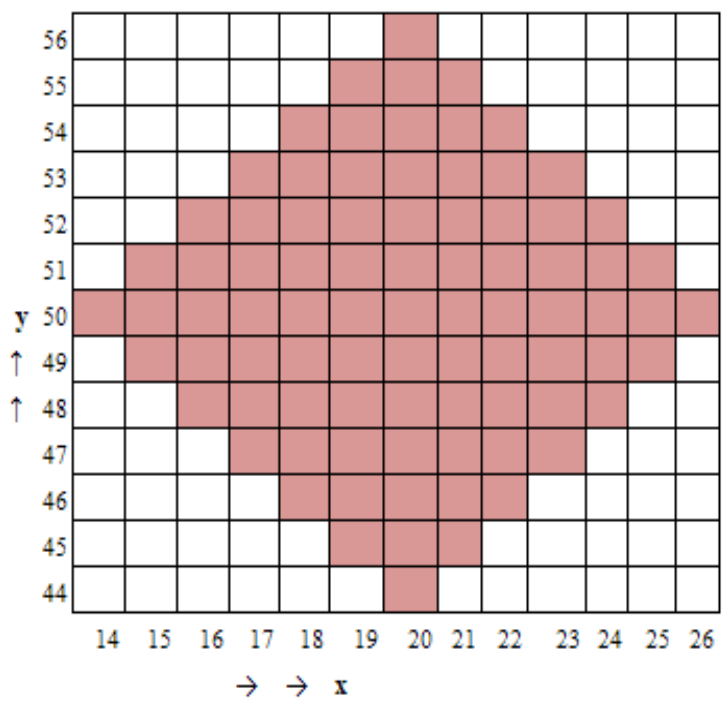

Figure 5-5 Tumor zone

Assuming the bio-thermal values for $k=0.52 \mathrm{~W} /(\mathrm{m} . \mathrm{K}), \omega_{b}=5.2 \times 10-41 / \mathrm{s}, Q_{m}=$ $700 \mathrm{~W} / \mathrm{m}^{3}$ for healthy tissue and $k=0.72 \mathrm{~W} /(\mathrm{m} . \mathrm{K}), \omega_{b}=2.2 \times 10-41 / \mathrm{s}, Q_{m}=40000 \mathrm{~W} / \mathrm{m}^{3}$ for tumour and the blood specific heat, and density as given in Table 5-7, the numerical simulation was performed using FDM and finite element approach for the spherical tumour of diameter 13
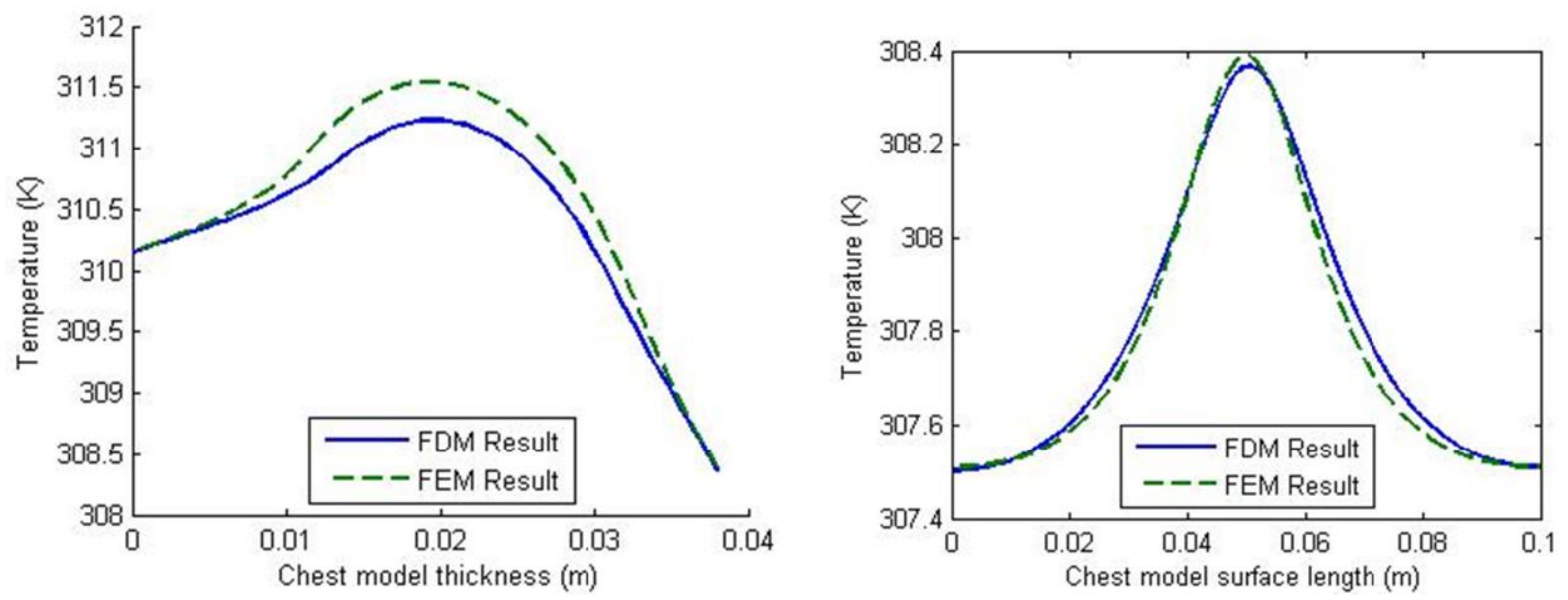

Figure 5-6 Comparison of numerical analysis results; plot of-(a) radial temperature flow between core and skin through tumor center, and (b) surface temperature between side to side opposite points right above the tumor centre

$\mathrm{mm}$ and located at $(0.02,0.05,0.05)$ on the proposed heterogeneous (two layer) model. The validation process includes a relative study of the 1D and 2D outward thermal flow in tissue inside and the spatial thermal distribution over skin surface. 


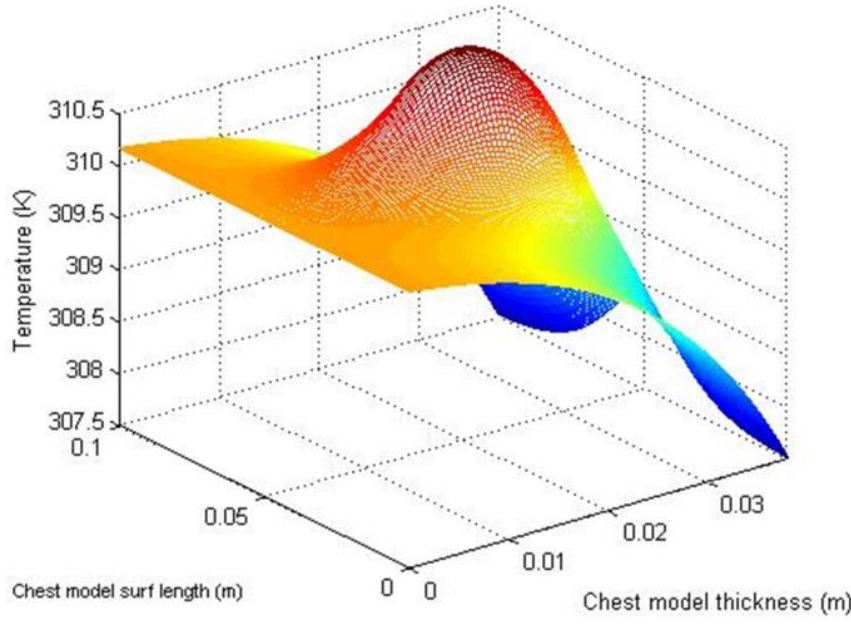

(a)

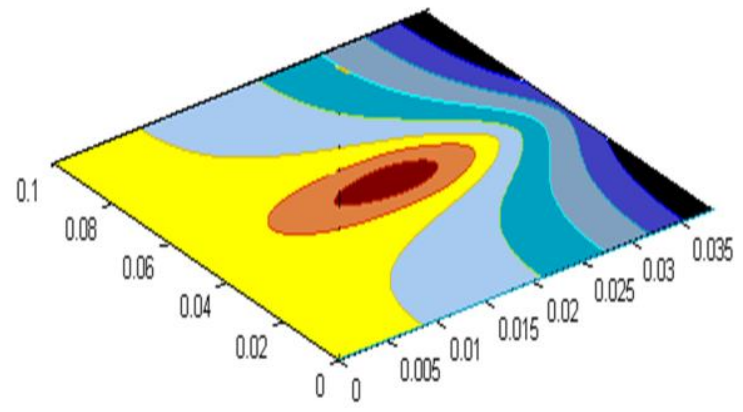

(c)

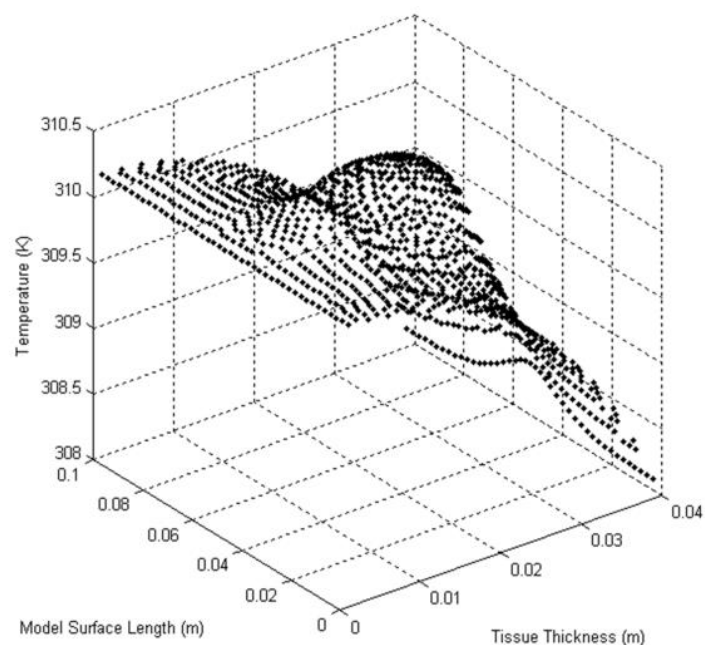

(b)

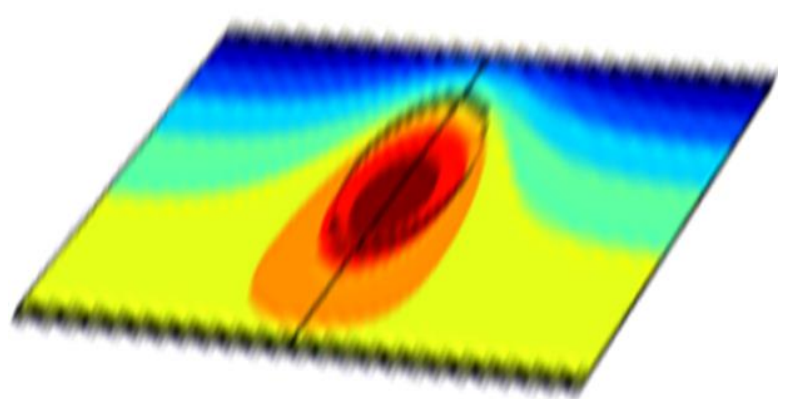

(d)

Figure 5-7 Comparison of numerical analysis results; for interior temperature distribution-(a) using FDM and (b) using FEM, on the horizontal plane through tumor center; and isothermal regions - (c) using FDM and (d) using FEM on the same plane 
The temperature flow diagram in tissue inside (along the line $\mathrm{y}=\mathrm{z}=0.05$ ) is presented in Figure 5-6(a) where the 'solid line' indicates the result given by the FEM and the 'dotted line' indicates the result of the FDM. Both curves have overlapped trimmings though an insignificant error $(\Delta T<0.5 \%)$ observed inside the tumour region. Evolution of temperature along $\mathrm{x}=0.04, \mathrm{y}=$ $0.05,0<z<0.1$ at skin surface is shown in Figure 5-6(b). It can be seen that both curve has symmetry. This figure also indicates that the warmest zone (surrounding the vertex), the main informative and significant point on the thermal image, has almost identical shape. The graphs
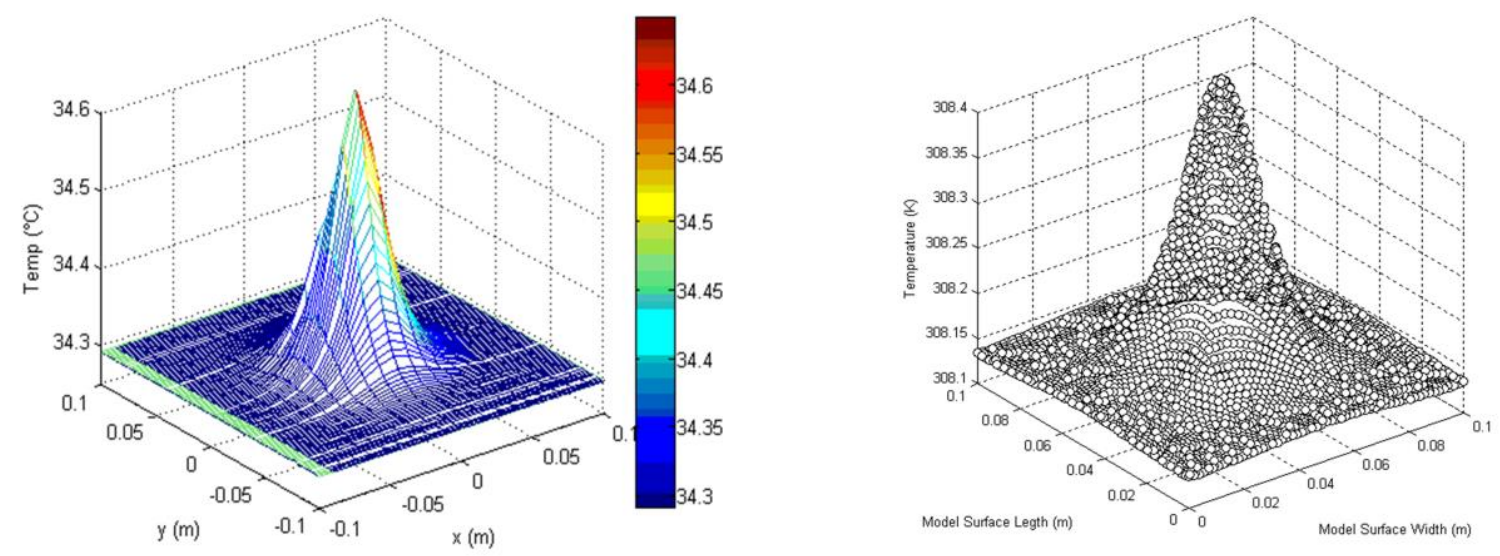

Figure 5-8 Spatial temperature distribution over chest skin surface

show that the drop in heat flow occur in both sides from the warmest point, the hottest zone on surface, indeed, lies directly above the tumor center. Note that the discrepancy between the approaches can be minimized by choosing smaller $h$ and $\varepsilon$ in FDM. In hyperthermia (i.e. tumor ablution with heating up/cooling down the tissues), the estimation of the thermal distribution inside tissues is essential. The spatial temperature distributions inside tissues (along plane $\mathrm{y}=0.05$ ) are obtained for both methods as shown in Figure 5-7. The graphs exhibit temperature grows, termed as 'temperature hill', on skin surface where the region subtended by the hill is caused for hyper metabolism of tumor cells and the subtend area is simply the temperature gradient filed distribution. The 'temperature hills' (red circles), shown in Figure 5-7(c) and (d), respectively for FDM and FEM analysis, are identical and the subtended area under the hills are alike. In hyperthermia, the estimation of the thermal distribution inside tissues is essential.

Unlike regular thermal distribution on a healthy organ, the tumor produces abnormal distribution with a relatively higher temperature over the skin surface above the area under which 
hyperactivity exists and the temperature increase estimated with the above tumor is shown in Figure 5-8, using FDM and FEM methods. The identical figures reveal the local warmest zones above the surface under which cancer cells are growing. Obviously, the features (vertex and warm zone) on skin temperature distribution will play a significant role in determining physio-thermal properties of tumor (indirect approach).

From above analyses, it is clear that the estimated profiles using FDM and FEM method are consistent. It can be concluded that either analysis can be used for the simulation efforts. However, negligible discrepancies are observed between two approaches which are originated due to the limitations in defining the node locations, the convergent criteria etc. which affect the FDM result, while the results obtained from the FEM method remain reliable unless the mesh size is compromised. In conclusion, it is demonstrated that the use of a commercially available heat transfer module provides a powerful tool to solve bio-heat problems for live biological tissues. Therefore, the onward study will apply the FEM method to address thermal problems. 


\subsection{Simplified vs. Realistic Models}

Bio-heat transfer problems on human external body organs, more specifically on the chest, forearm and breast have been solved using FDM and FEM where a Matlab script is written for FDM whereas a commercial solver is utilized for FEM. A comparative study has been made in section 5.6 to validate the built-in 'Bio-heat Transfer Model' of the FEM Analyzer. Another important issue to be investigated in this section is why developing realistic (anatomically accurate) physical models are necessary for accurate thermal analysis. For this, the study has developed simplistic models for the chest, forearm and breast along with the realistic (anatomicaccurate) models proposed in Chapter 3 (see Figure 3.6, 3.8, and 3.10). The simplistic model is also known as homogeneous model as the organs are assumed made with muscle tissues only. 
(a) $-\mathrm{i}$

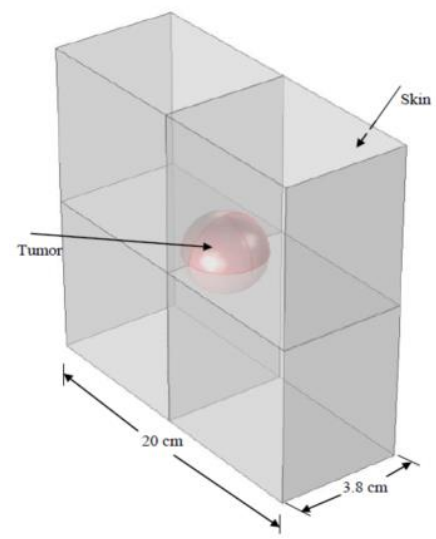

(a)-ii

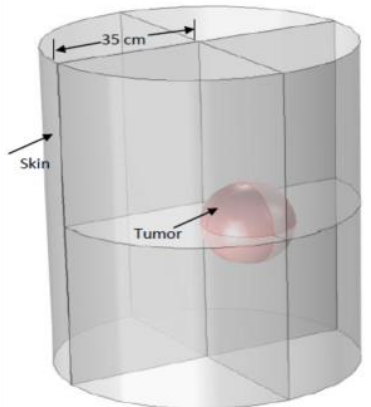

(a)-iii

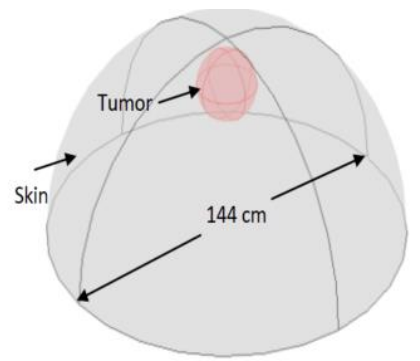

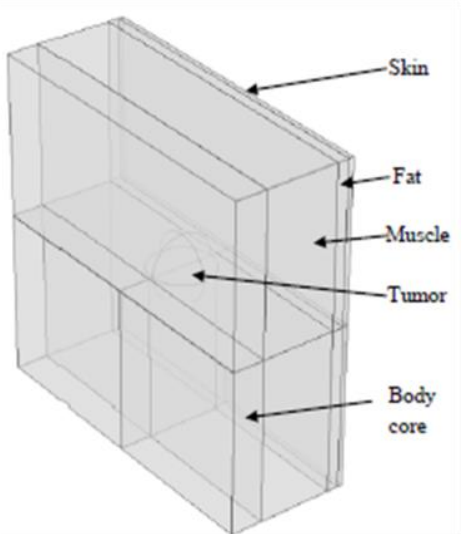

(b) $-\mathrm{i}$

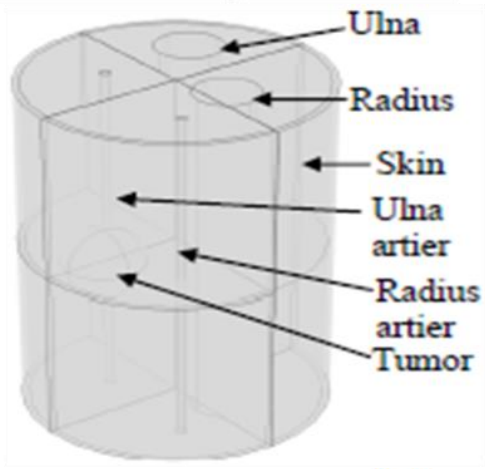

(b)-ii

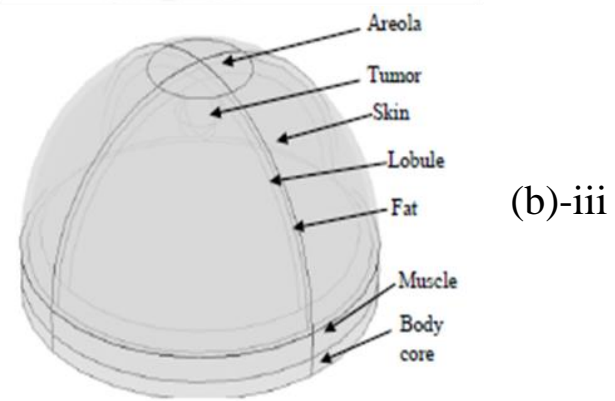

Figure 5-9 Physical models - a) homogeneous, and b) anatomic-accurate for tumor in-(i) cuboid (chest), (ii) tubular (forearm), and (iii) hemispherical (breast) organ

How to generate anatomic accurate physical models, mimicking the organ's structure (outlook and heterogeneity), was discussed in Chapter 3. Further study in Chapter 1, discussed the importance of imitating the organ's shapes (outlook) in developing the models. Recall, the analysis showed that the curved organ's (hemispherical model) surface senses colder than the tubular organ (cylindrical model) and the tubular organ feels less warm than the flat organ (cuboid model) even if all the thermo-physical parameters are assumed the same. In addition to the previous analysis, this section will present the obligation of imitating organ's non-homogeneity in developing realistic model which will come up with separate analyses on overly simplistic (single tissue) and realistic (multi-tissue) models. Thermal estimation over the overly simplified 3D rectangular 
model developed above and over anatomical accurate physical model will be explained separately with applying the 'FEM Analyzer'.

(a)-i

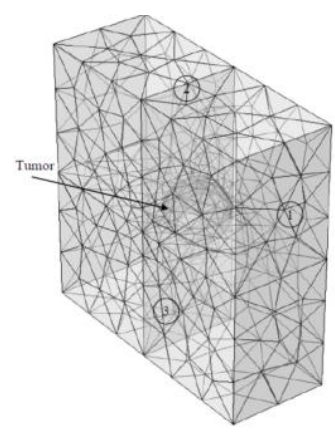

(a)-ii

(a)-iii
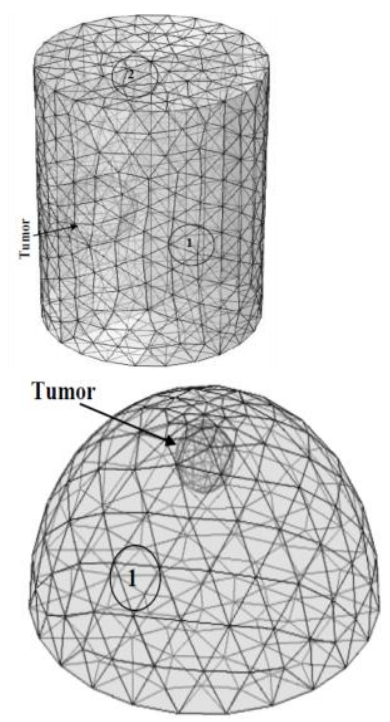

(b)-i

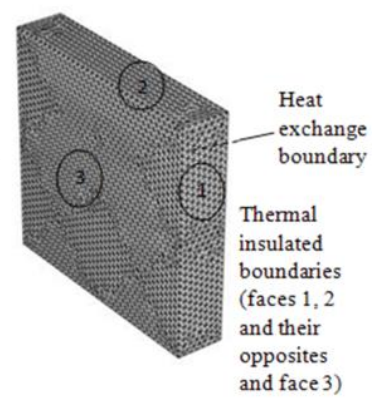

(b)-ii

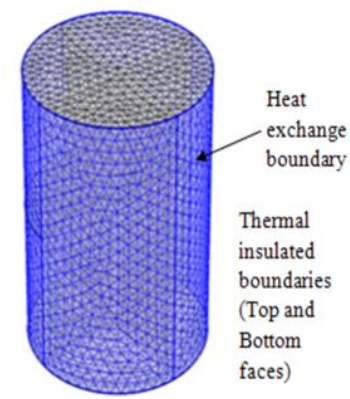

(b)-iil

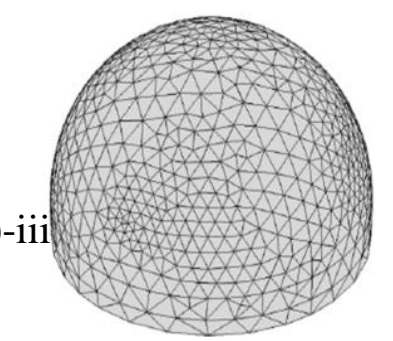

Figure 5-10 FE structure of - a) homogeneous, and b) anatomic-accurate models for tumor in-(i) cuboid (chest), (ii) tubular (forearm), and (iii) hemispherical (breast) organ

The simplistic physical models of the chest, forearm and breast are presented in Figure 59(a) and the realistic models of the similar organs are shown in Figure 5-9(b) where the (i) chest, (ii) forearm, and (iii) breast have been created with consideration of the entire domain made with single layers of tissues and the sensible models of these organs, respectively, notable that according to model proposed in Figure 4.6, Figure 4.8 and Figure 4.10, respectively. The dimensions and layer names are labelled on the figures.

Thermal analyses will be performed on both models in case of implanted hyperactive nodule where the implanted nodule buried in healthy soft tissues and behaves differently from the surrounding tissues in terms of heat conductivity, blood inflammation and metabolism as signs of hyperactivity. 
Though tumors may have different shapes (Figure 5- 1), only 'round' shapes with 'circumscribe' margins are assumed to avoid complexity that may arise due to the structural assortments in tumours. Assume an implanted chest tumor centered at $(0.02,0.05,0.05)$, the forearm tumor centered at $\left(0.023,90^{\circ}, 0.05\right)$ and the breast tumor centered on $\mathrm{z}$-axis and located at $z=0.065$.

The overall dimensions and thicknesses of each layer in physical model are defined accordingly described in Section 0. Notice that in the proposed model the geometry is separated into layers, however, while developed with FEM the domains (layers) are also segmented through horizontal and vertical planes at the center of tumour, the purpose such fragmentation is to explore the thermal flow along the inter-domain edges handily. It is noting that such virtual separate segments within a particular layer will be assigned with unique thermal and biological character so that they observe as a single domain.

Next step is generating meshes by disintegrating the physical model into finite number of polyhedral or polygonal elements. The FEM divides the model into small elements of geometrically simple shapes, generally tetrahedrons except triangular meshes at boundaries and edges. In each tetrahedron, a set of polynomial functions is used to approximate the structural displacement field - how much the object deforms in each of the three coordinate directions. In this study, because the tumour is relatively small in size, finer meshes are suggested. This would better resolve the thermal variations and give a more accurate result. A finer mesh, however, comes at a cost: the computation time as well as memory usage will go up. Choosing a mesh size is always a trade-off between accuracy on the one hand and speed and memory usage on the other hand. Mesh diagram of the models are shown in Figure 5-10 where the mesh for simple models is shown in (a) and of realistic models are in (b). The homogenous chest is disintegrated into approximately 27000 tetrahedral and 3500 triangular elements (Figure 5-10(a)-i); similarly, the simplified forearm model is divided into more than 200,000 tetrahedral elements and 28,000 triangular elements (Figure 5-10(a)-ii) and simple breast model with tumour is divided into approximately 5060 tetrahedral and 800 triangular elements (Figure 5-10(a)-iii). The same element size and aspect ratio have been applied to the realistic models which ended up with the tetrahedral and triangular elements approximately 10000 and 4500; 55000 and 16000; and 21000 and 7000; for the chest, forearm and breast model, respectively. The mesh diagram of chest model is shown in Figure 5-10(b)-i, forearm model in Figure 5-10(b)-ii, and breast model in Figure 5-10(b)-iii. In 
addition, nodes along the surface area constrained in the normal-translational direction for all geometries. All other nodes were unconstrained in all directions. The maximum and minimum element sizes were chosen as 0.6 and $0.2 \mathrm{~mm}$, respectively. The maximum element aspect ratio was 1.5 .

Second order heat transfer problem in living biological tissues is solved at steady-state. After assigning respective bio-thermal parameter values to the domains and attributing appropriate boundary conditions, a constant temperature of $293.15 \mathrm{~K}$ is applied at the entire section as an initial condition.

\subsection{Necessity of Anatomic-accurate (Realistic) Models}

For demonstrating the importance of developing the anatomic accurate physical model over the simplistic model, the study has derived thermal features of realistic and simplistic models. The thermal study compares the following features:

(i) Radial heat flows between body core and outer surface along the tumour center (i.e. tissue interior thermal graph)

(ii) $1 \mathrm{D}$ temperature distribution on the surface along the line that is passing directly above the tumour center (surface temperature graph)

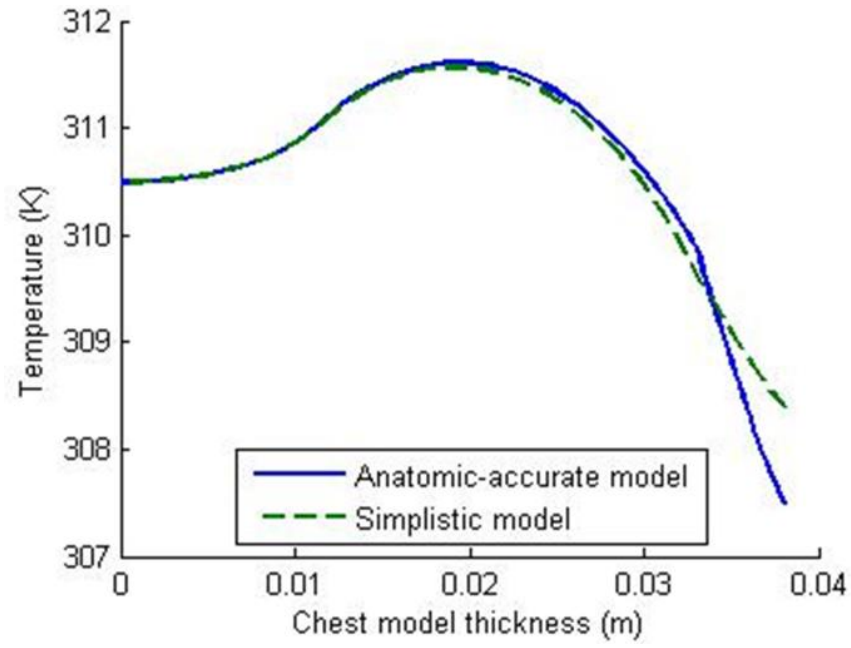

(a)

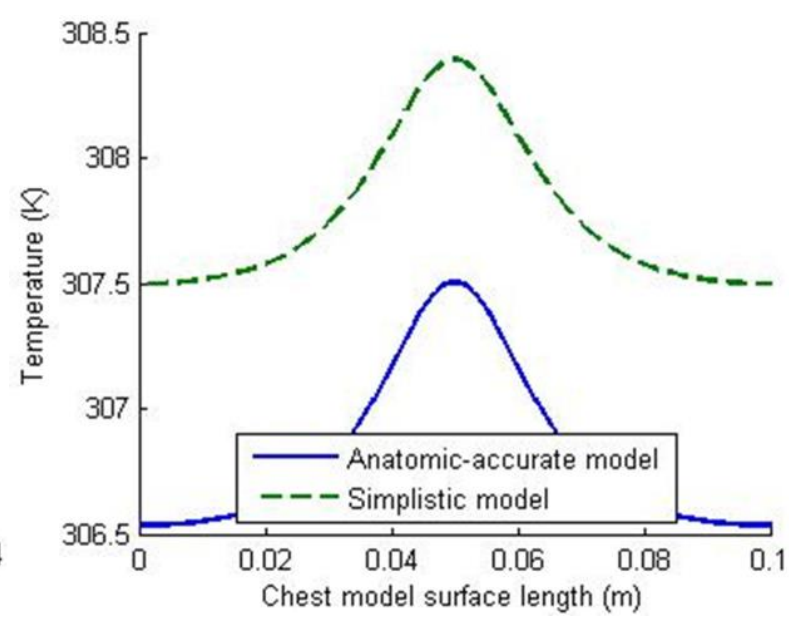

(b)

Figure 5-11 Comparison of temperature profiles obtained for a tumor in anatomic-accurate (realistic) and simplistic (homogeneous) chest model along the line-(a) between the body core and skin through tumor center, and (b) over the surface between two side to side opposite points right above the tumor center

The estimated results are presented Figure 5-11through Figure 5-13. Figure 5-11(a), the thermal analysis on the chest model, temperature flow along the line crossing the tumor center is 
shown where the 'solid line' and 'dotted line' present the temperature flow in simple and real model, respectively. The graphs are overlapped at the middle but anatomic-accurate model estimates lower temperature at both ends, especially at skin surface the temperature fall is quite clear. It can be said that both models can estimate temperature flow accurately in the defective region (around location of tumour), and, therefore, either model can be applied to the hyperthermia tumour ablution. On the other hand, the lower thermal coefficient of skin and fat tissues reduces the temperature by $0.8 \mathrm{~K}$ at skin surface in the anatomical accurate model than the simplistic model. The temperature gap $(\approx 0.8 \mathrm{~K})$ is maintained everywhere on the surface which is shown clearly in Figure 5-11(b). It is also observed that the shapes of the graphs are identical only shifted by $0.8 \mathrm{~K}$. The analyses have been assumed for a spherical tumor diameter $D=2 R=18 \mathrm{~mm}$, depth, $h=$ $1.7 \mathrm{~cm}$. The hyperactivity of cancerous cells have $\omega_{b}=1.6 \times 10^{-2} 1 / \mathrm{s}, \nabla k=0.1 \mathrm{~W} /(\mathrm{m} . \mathrm{K})$ and $Q_{m}=25 \mathrm{~kW} / \mathrm{m}^{3}$. Healthy tissue bio-thermal parameters, the ambient conditions and heat exchange rate are taken from Table 5-7.

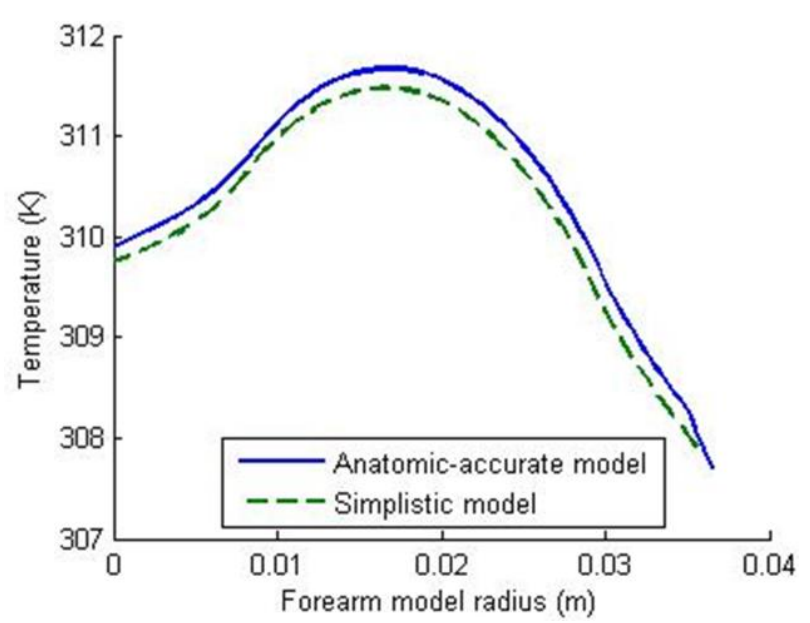

(a)

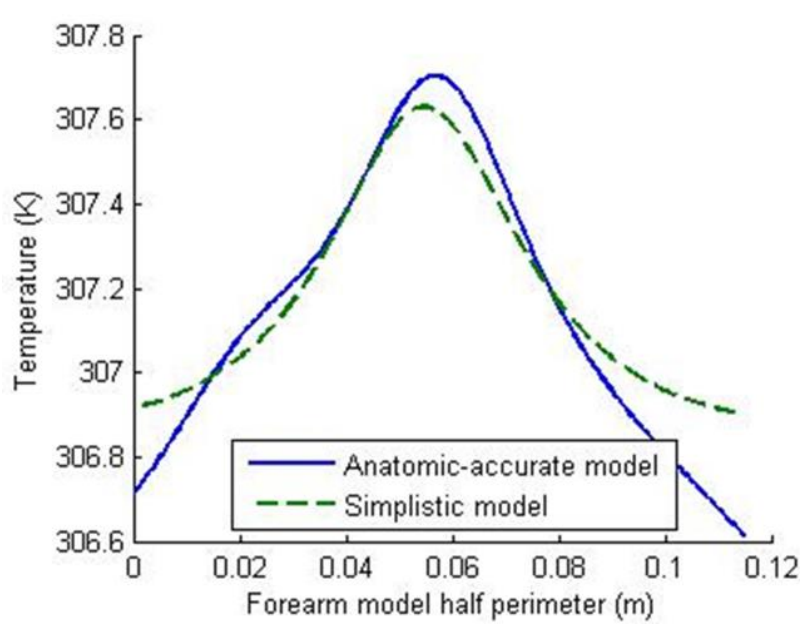

(b)

Figure 5-12 Comparison of temperature profiles obtained for a tumor in anatomic-accurate (realistic) and simplistic (homogeneous) forearm model along the line - (a) between the body core and skin through tumor center, and (b) over the surface between two side to side opposite points right above the tumor center

Assume a spherical tumor of diameter, $D=2 R=24 \mathrm{~mm}$, depth, $h=2 \mathrm{~cm}$ grown at the middle (z-axis) of the forearm model and considering the $\omega_{b}=2.2 \times 10-21 / \mathrm{s}, k=$ $0.62 \mathrm{~W} /(\mathrm{m} . \mathrm{K})$ and $Q_{m}=25 \mathrm{~kW} / \mathrm{m}^{3}$. Estimations indicate that the average gap between the plots is $|\Delta T|<0.001^{\circ}$ in model inside (see Figure 5-12(a)). Certainly, the absence of 'low $k$ ' fat layer in the forearm anatomy, however, estimates identical temperature elevation at skin surface 
for both models while the presence of bone tissues (radius and ulna) and the blood capillaries (radius and ulna arteries) distorts the temperature texture and the real model's surface temperature losses its symmetry, which may be a significant feature for correlating tumour with surface temperature.

The breast fat layer also influences the magnitude of maximum temperature elevation (see

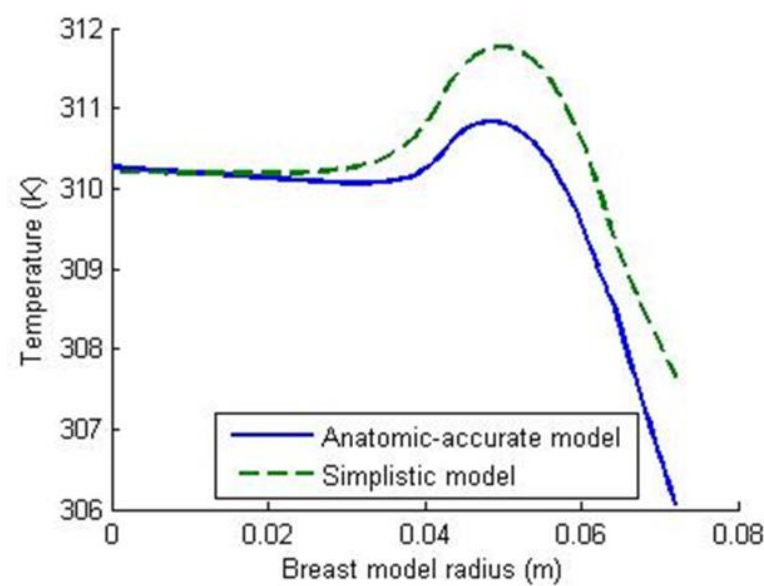

(a)

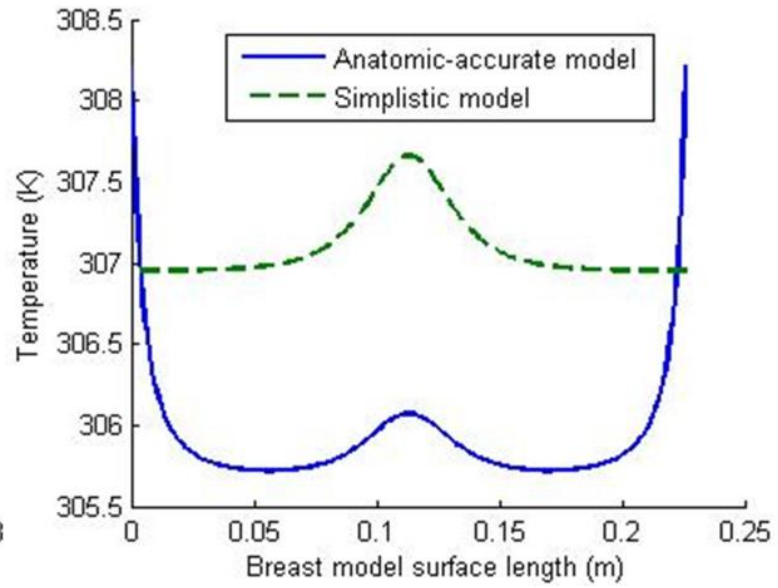

(b)

Figure 5-13 Comparison of temperature profiles obtained for an on-axis tumor in anatomicaccurate (realistic) and simplistic (homogeneous) breast model along the line - (a) between the body core and nipple, and (b) over the surface between two side to side opposite points through the nipple

Figure 5-13(a)) as well as pushes down the profile of the skin temperature distribution (see Figure 5-13 (b)). Figure 5-13(a) reveals that simple model estimates a maximum $308.58 \mathrm{~K}$ temperature on skin surface above the tumor center while the anatomical accurate model estimates the highest temperature $307.22 \mathrm{~K}$ for an on-axis spherical tumor having the same bio-thermal values of the chest tumor with $R=12 \mathrm{~mm}, h=17 \mathrm{~mm}$. It is seen from Figure 5-13(b) that for the simplistic models the 'temperature hill' is grown around the vertex and drops nonlinearly away from the vertex, however, for real model similar plot shows a sharp rise in temperature at the model base and then drops and again goes high to develop the 'hill' due to the tumor.

The major goal of the study is to correlate the thermo-physical parameters of a tumor with the featuring abnormal temperature grow on the surface. Exact thermal analysis is the sole requirement to correlated physical and thermal parameters, which can only be achieved on the anatomically accurate models. The above discussion has also made it clear that the simplistic models (homogeneous models) provide pessimistic results in terms of the shape and magnitude of 
temperature grow and thus might give poor estimate of the location, size and hyperactivity of tumor.

In conclusion, taking the realistic physical models, setting the proper boundary conditions, defining the accurate bio-thermal values are some preconditions to achieve good result. Thermal response of the realistic models for tumours with different thermo-physical values is going to be discussed next.

\subsection{Thermal Analysis}

This section will discuss the temperature distribution over local skin surface of the three organs in presence of implanted tumors. The temperature profiles on the surface of the proposed realistic models of the organ with faulty region, (tumor) where the faulty region having different physio-thermo-biological features, are being investigated. The analyses mostly cover studies of malignant and benign; deep-seated and shallow; fast and slow growing; on-axis and off-axis tumors etc. Depending on the parameter's value range, tumors are termed as benign (radius between 6 and $15 \mathrm{~mm}$, heat rate between $5 \mathrm{~kW} / \mathrm{m}^{3}$ and $25 \mathrm{~kW} / \mathrm{m}^{3}$ ), malignant (radius between 21 and $38 \mathrm{~mm}$, heat rate between $50 \mathrm{~kW} / \mathrm{m}^{3}$ and $80 \mathrm{~kW} / \mathrm{m}^{3}$ ), shallow body tumor (depth between $5 \mathrm{~mm}$ and $12 \mathrm{~mm}$ ) and deep seated tumor (depth more than $20 \mathrm{~mm}$ ). It is worth mentioning that the above categorization is still a debated issue among scientists and researchers. The analysis discovers the 2D surface temperature and 1D center-line temperature profiles where the center-line is the horizontal (Left-to-Right, L-R) and vertical (Bottom-to-Top, B-T) lines over the outer surface of the model center.

Numerical results of the thermal problem to the above models are presented through Figure 5-14 to Figure 5-19. Assuming the cancerous cells have $k=0.62 \mathrm{~W} /(\mathrm{m} . \mathrm{K}), \omega_{b}=0.0022 / \mathrm{s}$, four chest tumors are analyzed-Tumor 1, benign, diameter $D=12 \mathrm{~mm}$, submerged at $h=1.6 \mathrm{~cm}$ and $Q_{m}=20 \mathrm{~kW} / \mathrm{m}^{3}$; Tumor 2, small malignant, $D=12 \mathrm{~mm}, h=1.2 \mathrm{~cm}$ and $Q_{m}=50 \mathrm{~kW} / \mathrm{m}^{3}$; Tumor 3, deep-seated large malignant, diameter $D=20 \mathrm{~mm}, h=1.7 \mathrm{~cm}$ and $Q_{m}=70 \mathrm{~kW} / \mathrm{m}^{3}$; Tumor 4, large malignant, $D=12 \mathrm{~mm}, h=1.6 \mathrm{~cm}$ and $Q_{m}=80 \mathrm{~kW} / \mathrm{m}^{3}$. Figure $5-14($ a) to (d) show their respective 2D temperature distributions, where each graph is divided into several isothermal contours and Figure 5-14(e) shows 1D centerline (L-R or B-T) temperature profiles. It is discovered tumor-3 produces maximum $307.28 \mathrm{~K}$ above the center and if depth decreased by $6.25 \%$ and size by $78 \%$, elevates the apex by $1 \mathrm{~K}$ for only $14.28 \%$ increase of metabolism (Tumor 
4). In the first two cases, temperature raise is only $0.2 \mathrm{~K}$ may be screen by high sensitive IR images and would be impossible to accurate parameterize due to smaller dataset size they provide.

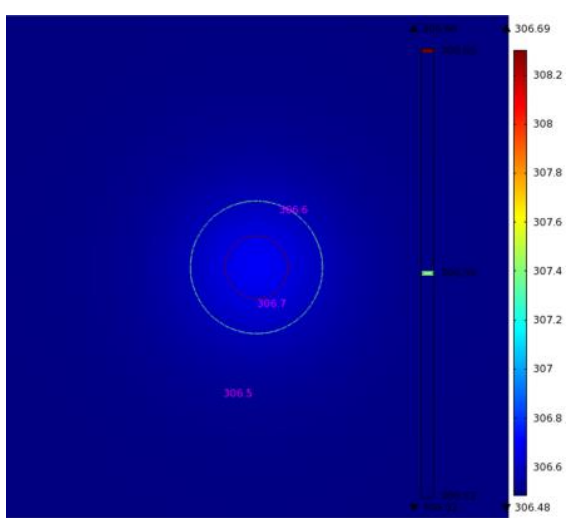

(a)

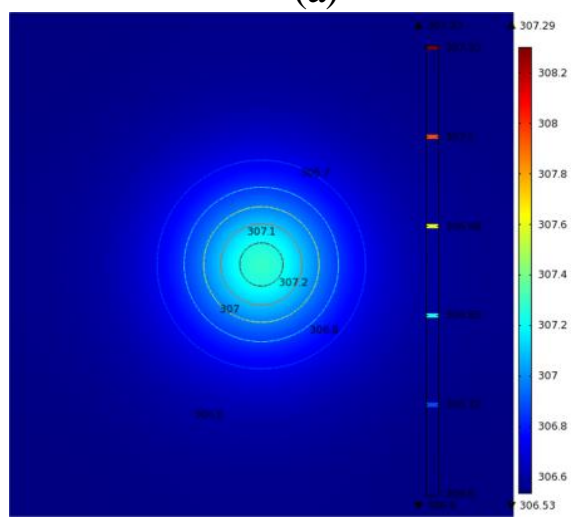

(c)

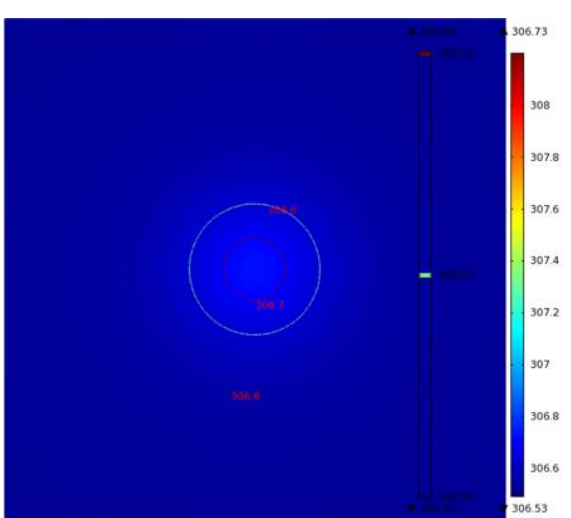

(b)

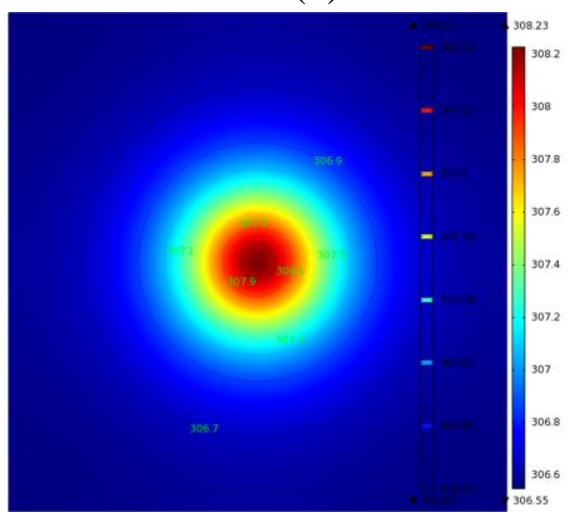

(d)

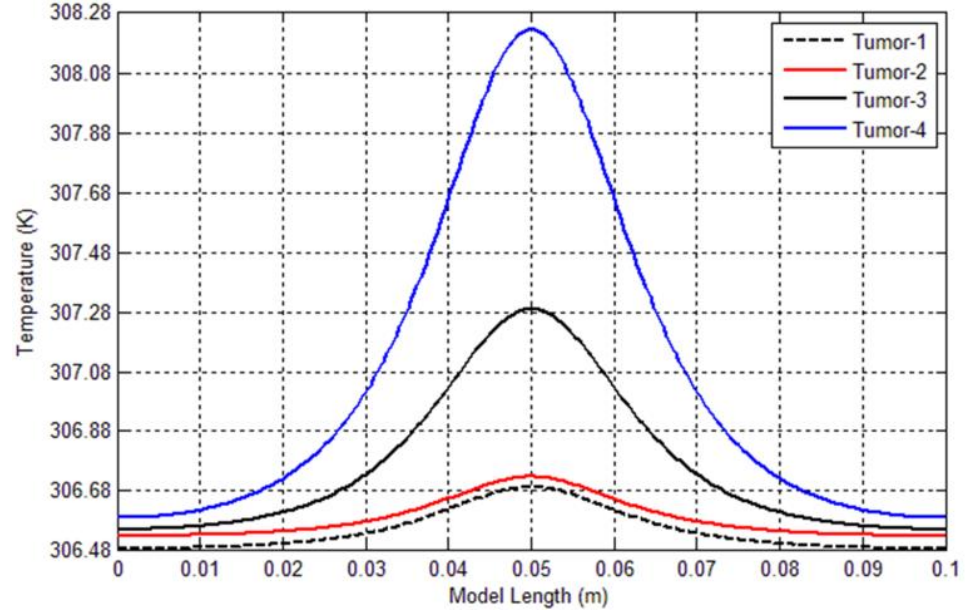

(e)

Figure 5-14 Chest tumors; (a) to (d) surface temperature for tumor 1 to 4, respectively, (e) center-line temperature

Most of the thermal sensing device can isolate a temperature contrast at least $0.2 \mathrm{~K}$, for this the study has investigated the skin area which feels at least $0.2 \mathrm{~K}$ hotter than the super cold region and 


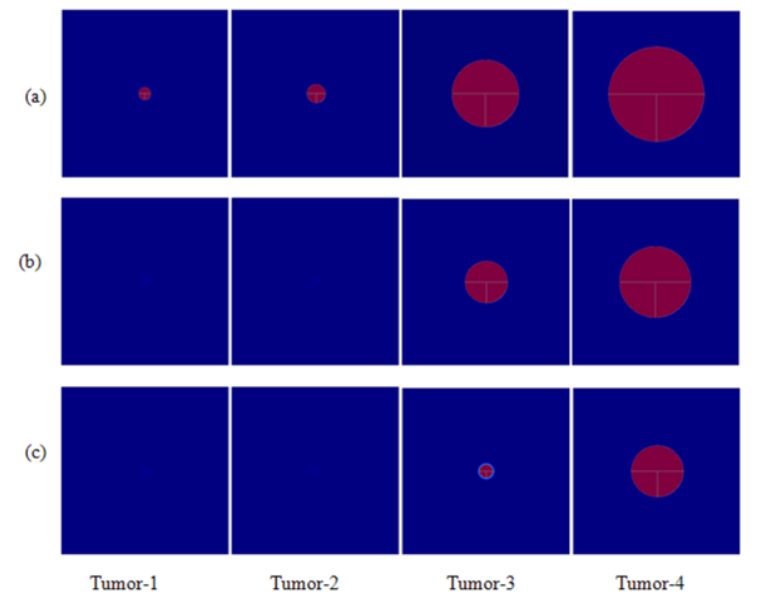

Figure 5-15 Area of temperature grow, (a) to (d) for chest tumor 1 to 4, respectively showed how the area is related with the physio-bio-thermal parameters of the tumor. The above four cases provide warmer zones as shown in Figure 5-15 where the 'red' colored $\Delta \mathrm{T} \geq 0.2 \mathrm{~K}$ region for each tumor shown in (a), $\Delta \mathrm{T} \geq 0.6 \mathrm{~K}$ in (b) and $\Delta \mathrm{T} \geq 0.8 \mathrm{~K}$ in (c) respectively, generally indicates the warm zone area is a function of $d, h$ and $Q_{m}$.

Assuming forearm model centered at $(0,0,0.05)$, the similar analysis is conducted onTumor 1, benign, $D=12 \mathrm{~mm}$, located at $(-0.019,0,0.05)$ and $Q_{m}=20 \mathrm{~kW} / \mathrm{m}^{3}$; Tumor 2 , malignant, diameter $D=20 \mathrm{~mm}$, located at $(-0.019,0,0.05)$ and $Q_{m}=80 \mathrm{~kW} / \mathrm{m}^{3}$; Tumor 3, deepseated large malignant, $D=24 \mathrm{~mm}$, located at $(-0.013,0,0.05)$ and $Q_{m}=80 \mathrm{~kW} / \mathrm{m}^{3}$; Tumor 4 , shallow-body large malignant, $D=24 \mathrm{~mm}$, located at $(-0.021,0,0.05)$ and $Q_{m}=50 \mathrm{~kW} / \mathrm{m}^{3} .2 \mathrm{D}$ surface temperature distributions of each tumor are presented in Figure 5-16(a) to (d), respectively, and 1D center-line (L-R) temperature is presented in Figure 5-16(e). Tumor 1 cannot make sensible alterations on the natural temperature distribution and could provide 'false negative' screenings. However, if the diameter increased by $66 \%$ and heat rates by three folds, the tumor at the same location produces almost $1.2 \mathrm{~K}$ elevation from the super cooled temperature. A tumor nearer to the skin surface has tremendous effect on surface temperature, a tumor right under the fat layer (tumor 4) feels 1.75K more warmer than a same size (tumor 3) 17.5 deep tumor with 1.6 times high rate in volumetric heat production. The shapes and warmest location are clearly observed from Figure 5-16(e), the curves reveal that if the temperature patters hold L-R symmetry, improve accuracy diagnosis. 


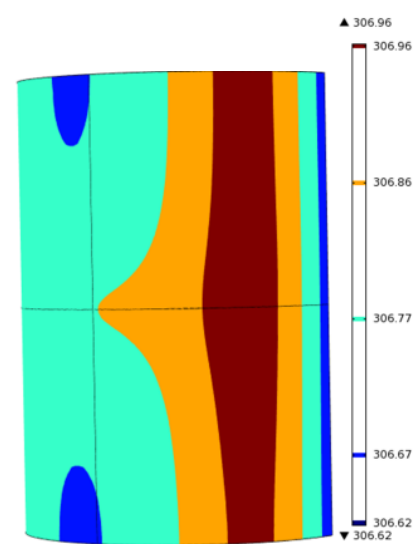

(a)

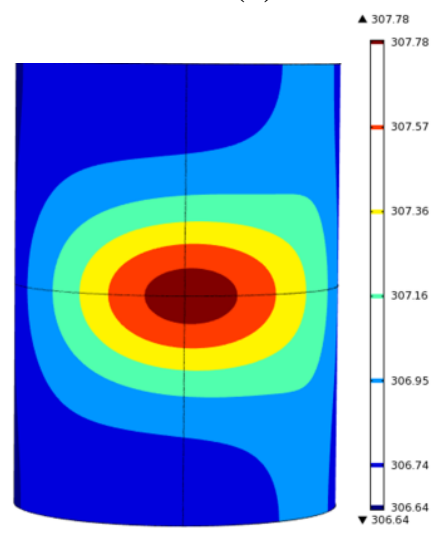

(c)

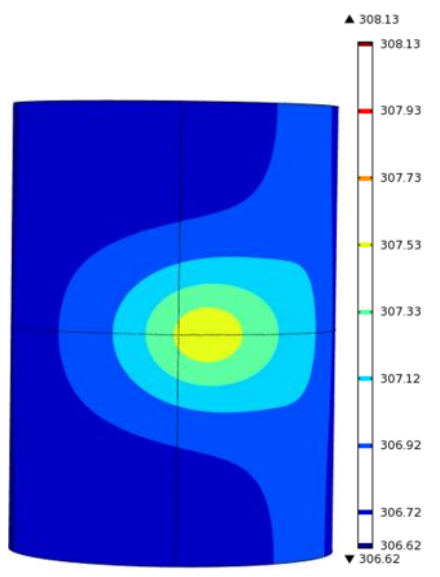

(b)

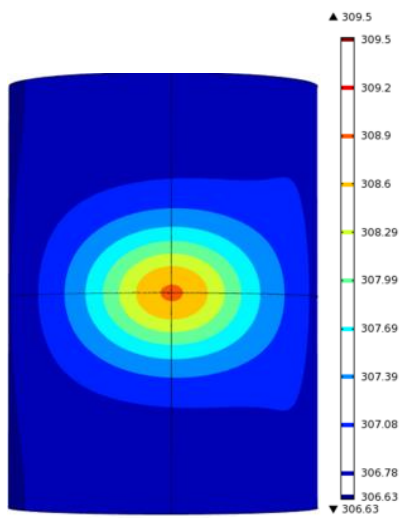

(d)

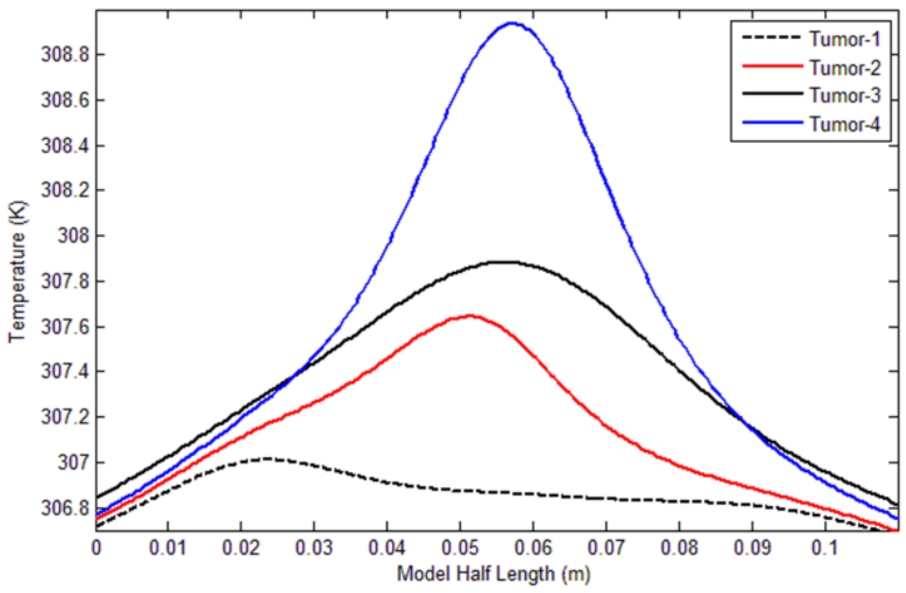

(e)

Figure 5-16 Forearm tumors, (a) to (d) surface temperature for tumor 1 to 4, respectively, (e) center-line (L-R) temperature

Warmer zones detected over the forearm due to these tumors are explored in Figure 5-17 121 
where (a) shows the region over which $\Delta \mathrm{T} \geq 0.2 \mathrm{~K}$ felt, (b) shows $\Delta \mathrm{T} \geq 0.4 \mathrm{~K}$ region and (c) shows $\Delta \mathrm{T} \geq 0.6 \mathrm{~K}$ region. It is clearly evident that the tumor 1 couldn't show any sign of its presence and remain untraced in thermogram, resulting in a false negative outcome. Though the defective zone is assumed spherical, the surface warm zone is not symmetrical over forearm.

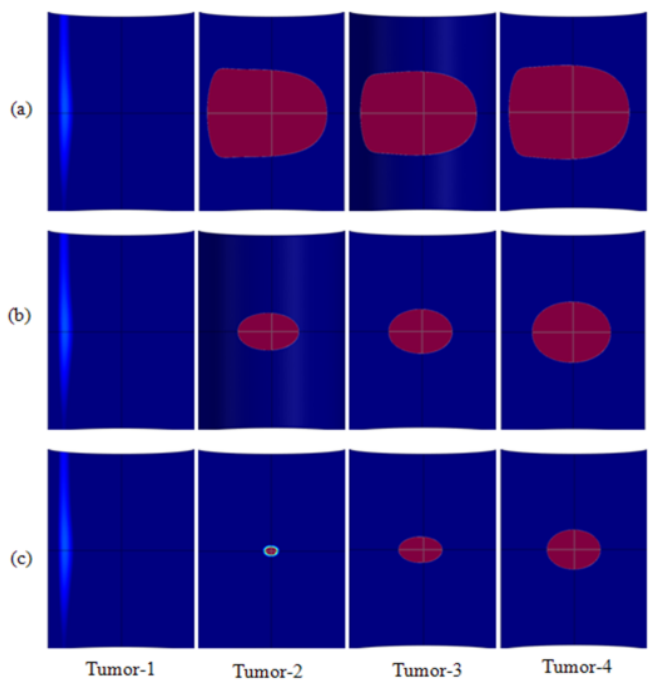

Figure 5-17 Area of temperature grow, (a) to (d) for forearm tumor 1 to 4, respectively

Breast tumor thermal analysis is performed for-Tumor 1 , benign $d=24 \mathrm{~mm}$ located at $(0$, $0,0.052), Q_{m}=40 \mathrm{KW} / \mathrm{m}^{3}$; Tumor 2 , deep-seated large benign $D=54 \mathrm{~mm}$ located at $(0,0$, $0.034), Q_{m}=40 \mathrm{KW} / \mathrm{m}^{3}$; Tumor 3 , small malignant $D=24 \mathrm{~mm}$ located at $(0,0,0.052), Q_{m}=$ $80 \mathrm{KW} / \mathrm{m}^{3}$; Tumor-5, deep-seated large malignant $D=54 \mathrm{~mm}$ located at $(0,0,0.034), Q_{m}=80$ $\mathrm{KW} / \mathrm{m}^{3}$; Tumor-5 malignant $D=24 \mathrm{~mm}$ located at $(0, \pm 0.017,0.052), Q_{m}=80 \mathrm{KW} / \mathrm{m}^{3}$; Tumor 6 big benign $D=24 \mathrm{~mm}$ located at $(0, \pm 0.017,0.052), Q_{m}=40 \mathrm{KW} / \mathrm{m}^{3} \mathrm{e}$. Assumed breast model is centered at origin and perfusion rate of tumors is $\omega_{b}=4.6 \times 10^{-3} 1 / \mathrm{s}$. Figure $5-18$ presents the 2D spatial temperature over surface for each case respectively in Figure 5-18(a) to (e) where on-axis tumors develops symmetric distribution (a-d) but loss symmetry in case of tilted tumors $(\mathrm{e}, \mathrm{f})$. The 1D center-line temperature plots for above cases are presented in Figure 5-19. It is seen that Tumor-1 and 4 produce very same profile, might lead non-unique inverse estimation. A breast tumor could produce more than $2 \mathrm{~K}$ higher temperature on surface. 


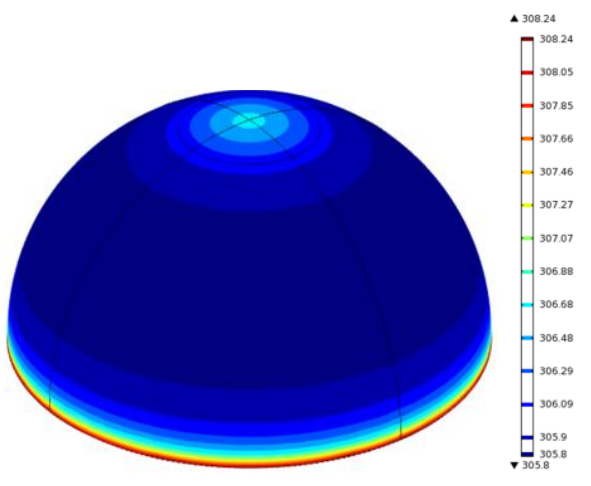

(a)

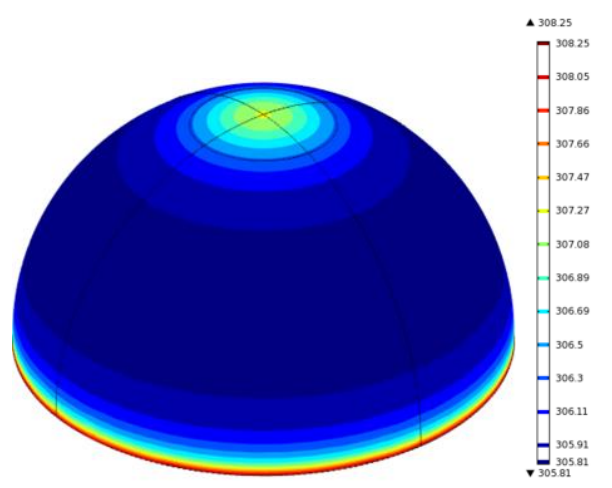

(c)

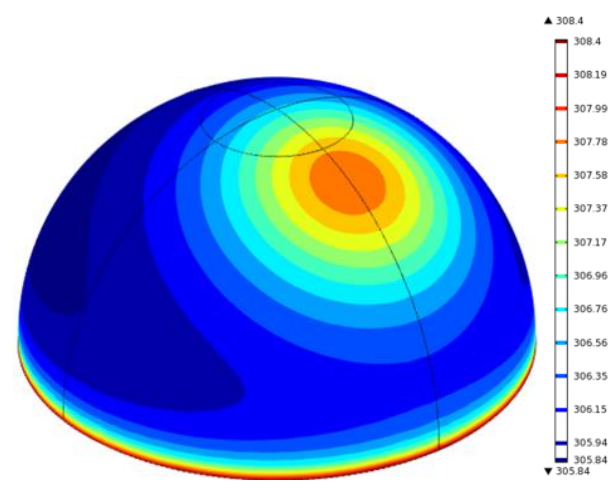

(e)

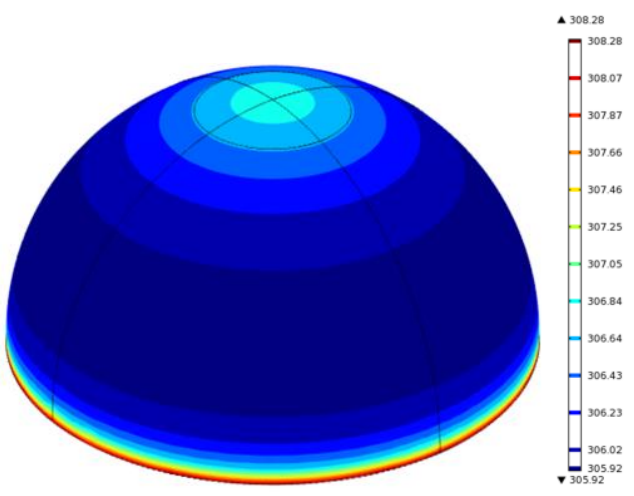

(b)

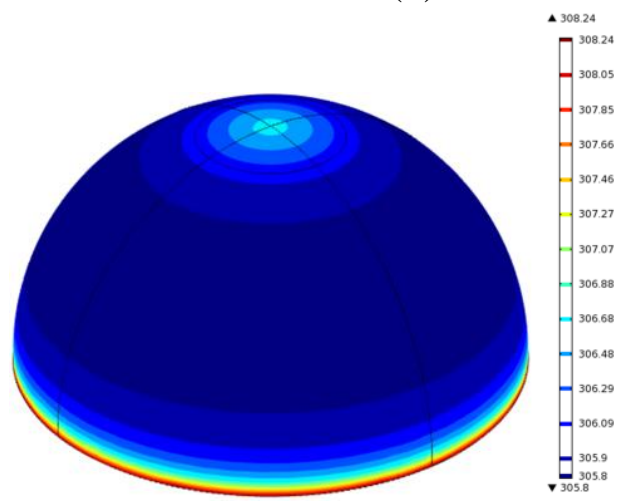

(d)

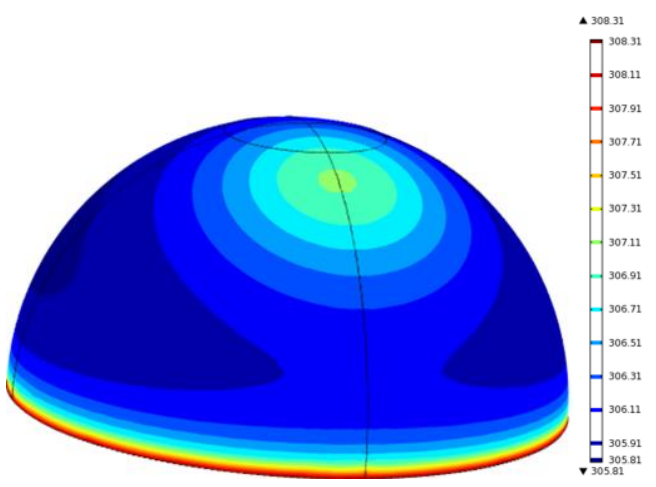

(f)

Figure 5-18 Breast tumor analysis; (a) to (f) surface temperature for tumor 1 to 6 , respectively

In the above analyses, thermal features of tumor in the chest, forearm and breast have been investigated while the physical parameters (such as size and location) and the bio-thermal parameters (such as conductivity, metabolism and perfusion rate) of the tumor are reported to have influences on the thermal features, to be exact, the temperature elevation and shape (or area) of warm zone. Therefore the analyses investigate the surface thermal feature as a function of size, 


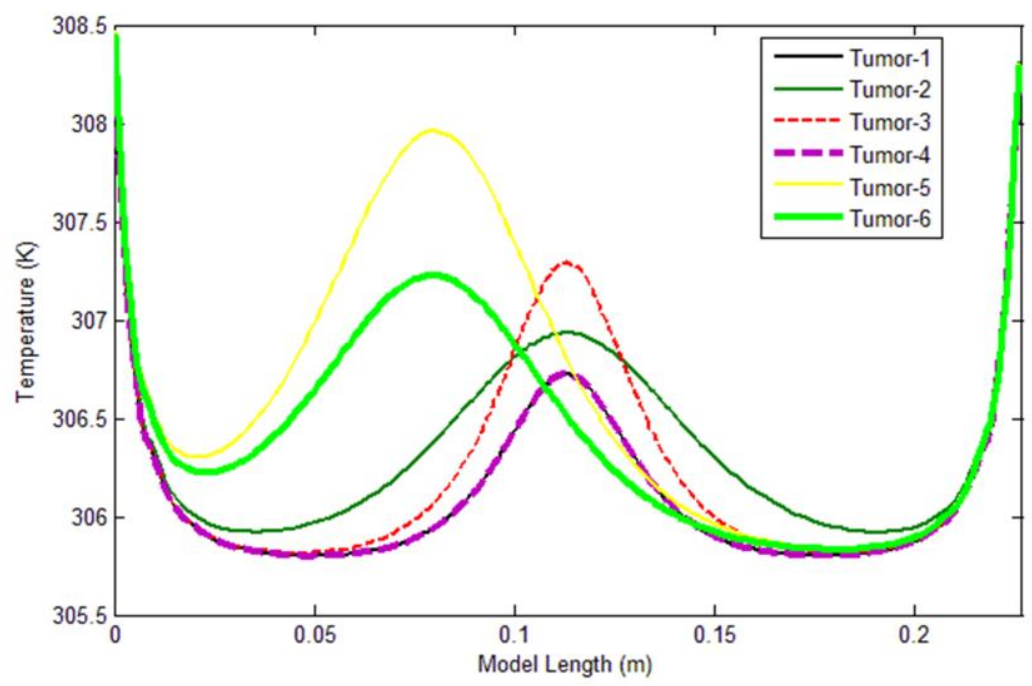

Figure 5-19 Center-line temperature for tumor in undeformed breast

location, conductivity, metabolism and perfusion. Of these variables, the size and location are independent but the conductivity, heat generation rate and perfusion rate are mutually dependent (see Eqn. 5.1 to 5.3). In the subsequent section the influence of physio-thermo-biological parameters will be computed. Moreover thermal feature also depends on the rate at which heat is exchanged with environment (see Eqn. 1.1) therefore the correlation between temperature graph and ambient conditions will also be explored.

\subsection{Effect of Physio-thermo-biological Parameters and the Surrounding}

The above analysis demonstrated that the abnormal surface temperature patterns over the faulty organ were controlled by biological thermal and physical parameters. The purpose of this section is to determine the impact of each controlling parameter on the temperature pattern. Equations 4.10-4.12, 4.27 and 4.46 show that steady-state temperature flow in tissue interior is a function of metabolism $\left(Q_{m}\right)$, blood perfusion rate $\left(\omega_{b}\right)$, blood specific heat $\left(C_{b}\right)$, heat conduction rate $(k)$, environment temperature $\left(T_{e}\right)$, heat exchange rate $\left(h_{a}\right)$ and model radial dimension (Figures 4.15 through 4.20 show how each of the above parameter influences the internal temperature flow of tissues). Further analysis in the previous section shows that surface temperature above the faulty organ is a function of the tumor's physical, biological and thermal values provided that the ambient remains unchanged. The analyses has categorized these parameters into three groups_-bio-thermal (metabolism, blood perfusion rate, blood specific heat, 
heat conduction rate) group, environmental (ambient temperature, heat exchange rate) group, and physical (model radial dimension, tumor size, and location) group. Inspecting Equation 5.1 through 5.3, it is seen that thermal conductivity is affected by perfusion rate and metabolism and size are influenced by the doubling time. Considering tumor's doubling time and incremental thermal conductivity are known, the other parameters are computed accordingly. Assume a tumor grown in $20 \mathrm{~mm}$ deep, doubles in 38 days and thermal conductivity increases by $0.2 \mathrm{~W} /(\mathrm{m}-$ $K)$ form healthy tissues $(k=0.52 \mathrm{~W} /(m-K))$. Therefore, the other parameters are computed to be-metabolic rate $85000 \mathrm{~W} / \mathrm{m}^{3}$ (see Eqn. 5.2) blood perfusion rate $0.161 / \mathrm{m}$-s (see Eqn. 5.1) and the diameter $\approx 10 \mathrm{~mm}$ (see Eqn. 5.3). It is worthless of sweeping model radial dimension and blood specific heat because tumor has no known effect on them. Influence of other variables will be inspected separately. 


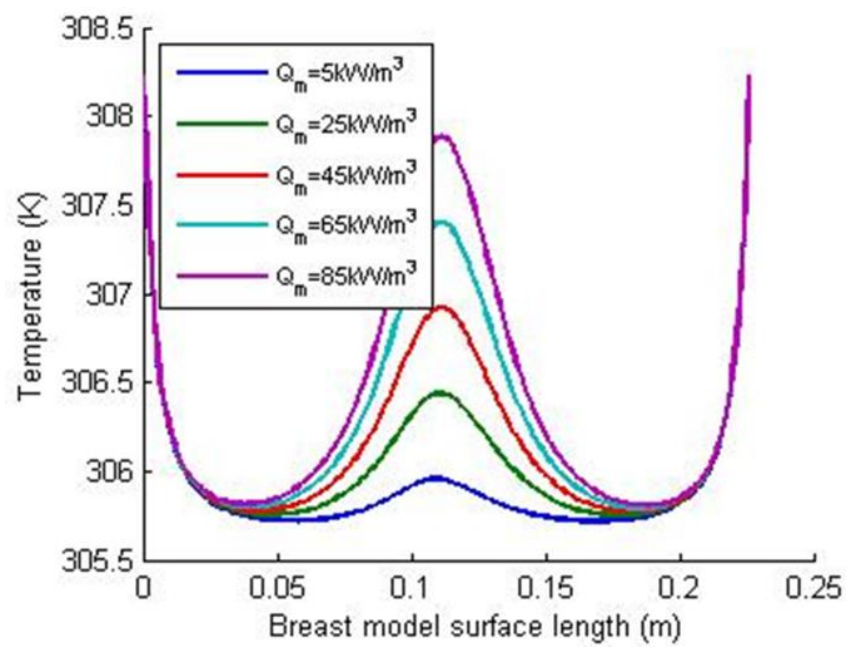

(a)

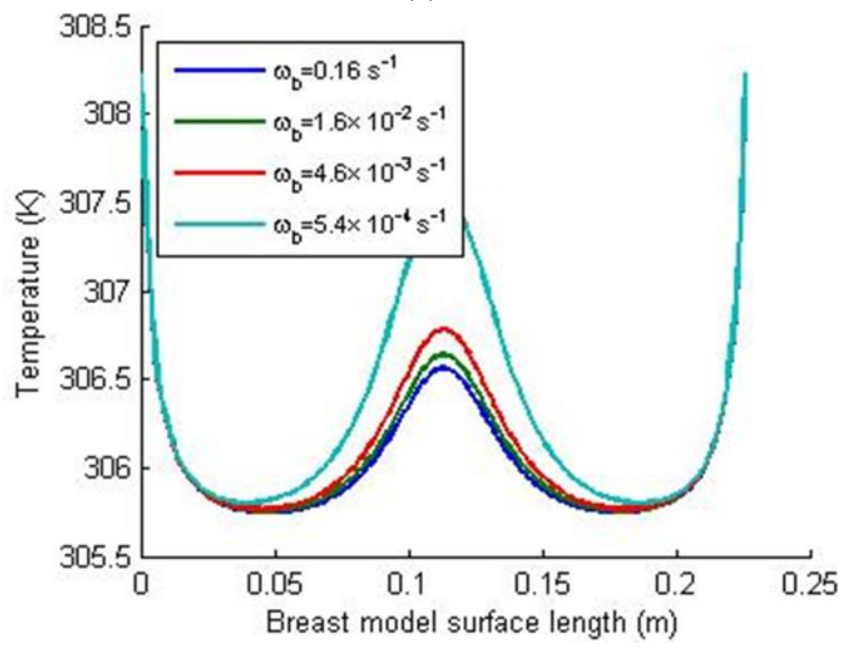

(b)

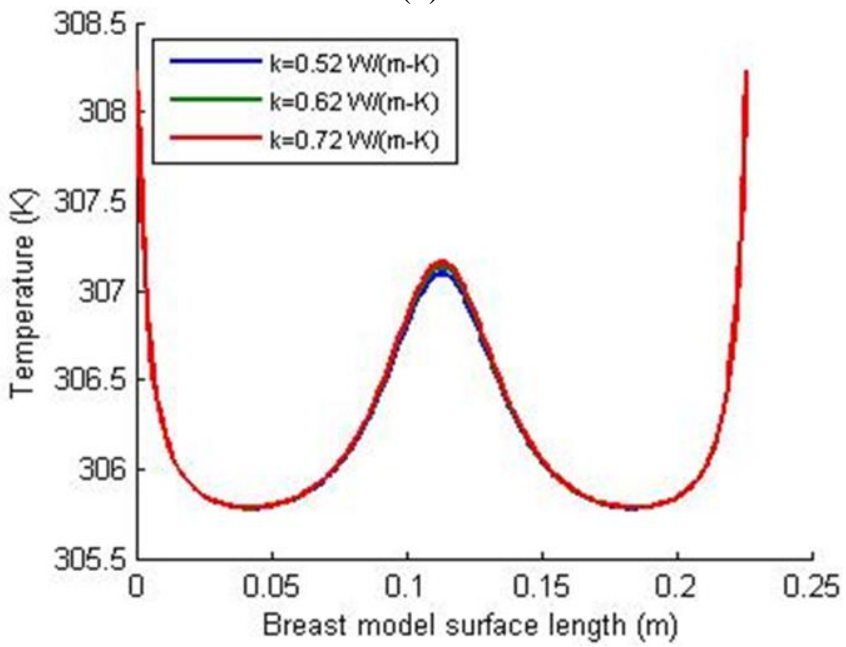

(c)

Figure 5-20 Effect of bio-thermal parameters of tumor, e.g. influence of - (a) metabolism, (b) perfusion and (c) heat conduction rate of tumor on breast surface temperature

For computing the parametric effect on spatial temperature distributions on realistic models, 
the study has performed parametric sweep. However, the report presents thermal computations of spherical on-axis tumour submersed in lobule layer in the natural un-deformed breast model only to exemplify the aspect.

Thermal values of healthy breast tissues are acquired from Table 5-7. Except the swept variable the other parameters of tumor have assumed $Q_{m}=25000 \mathrm{~W} / \mathrm{m}^{3}, h=2 \mathrm{~cm}, \omega_{b}=3.4 \times$ $10^{-4} \frac{1}{\mathrm{~s}}, R=1.5 \mathrm{~cm}$ as well as the heat losses rate is $h_{a}=13.7 \mathrm{~W} / \mathrm{m}^{2}$ and ambient temperature is $T_{e}=300.15 \mathrm{~K}$. The analysis follows-(i) estimation of the bio-thermal influences (Group-1), (ii) estimation of the pinpointing parameters' influences (Group-2) and (iii) estimation of the ambient influences (Group-3). Obtained thermal results are plotted along L-R line (a line joining two opposite left and right base points through model vertex) or T-B line (a line joining two opposite top and bottom base points through model vertex)for each case are the following:

The influences of bio-thermal group variables on surface temperature distribution are presented in Figure 5-20 through (a) to (c), respectively. The family of graphs in Figure 5-20 (a) while sweeping $Q_{m}$ between $5 \mathrm{k}$ and $85 \mathrm{k}$ in step of $20 \mathrm{k}$ per unit volume, presents the $1 \mathrm{D}$ temperature plot along L-R (or T-B) line. It is observed that the heat rate has a linear relationship with the maximum temperature elevation and the area over which the temperature grows. The opposite effect could be found for the perfusion heat loss rate, which is presented in Figure 5-20 (b) with the blood flow rate shown. Unlike the wide varying metabolic and perfusion rates, the swing in the conduction heat flow rate is insignificant, thus plays a worthless effect (see Figure 520 (c)) on the temperature figure.

The effect of the tumor's physical parameters (Group-2) on temperature pattern is shown in Figure 5-21. Thermal plots of tumor developed in different locations show that depth has a nonlinear relation with the heat flow and with the maximum temperature rise at skin, which is observed from Figure 5-21(a). It is evident that a deep seated tumor could give a false negative result at the earlier stage. In contrast, the size of a tumor is a linear function of temperature, i.e. the bigger tumor will elevate the temperature peak and increase the hot zone area (see Figure 5-21(b)).

How the environmental condition (Group-3) affects the temperature pattern is given in Figure 5-22-window (a) and (b) for ambient temperatures and heat loss rates, respectively. Inspecting the left figure it is clear that the heat exchange rate doesn't affect the shape of the graphs but rather pushes them downwards when increased (Figure 5-22(a)). On the other hand, the higher 


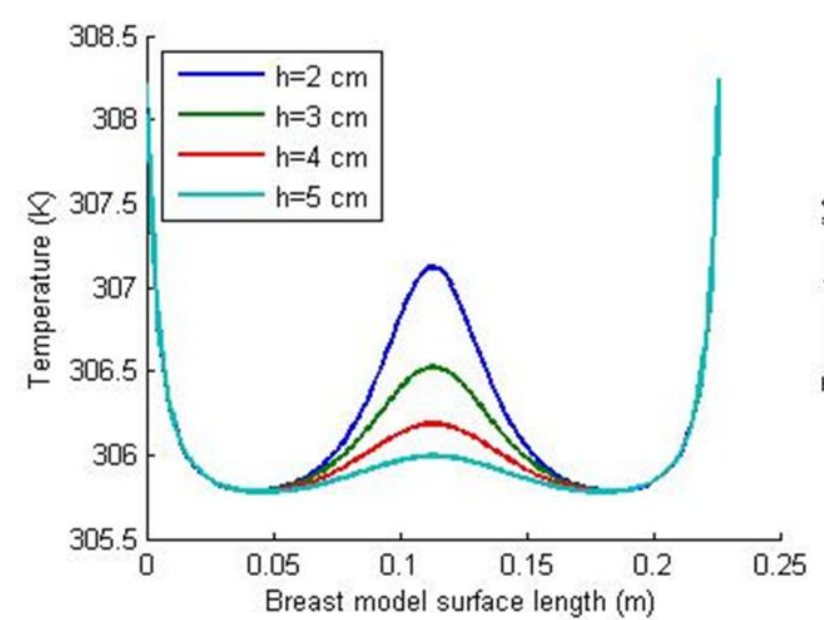

(a)

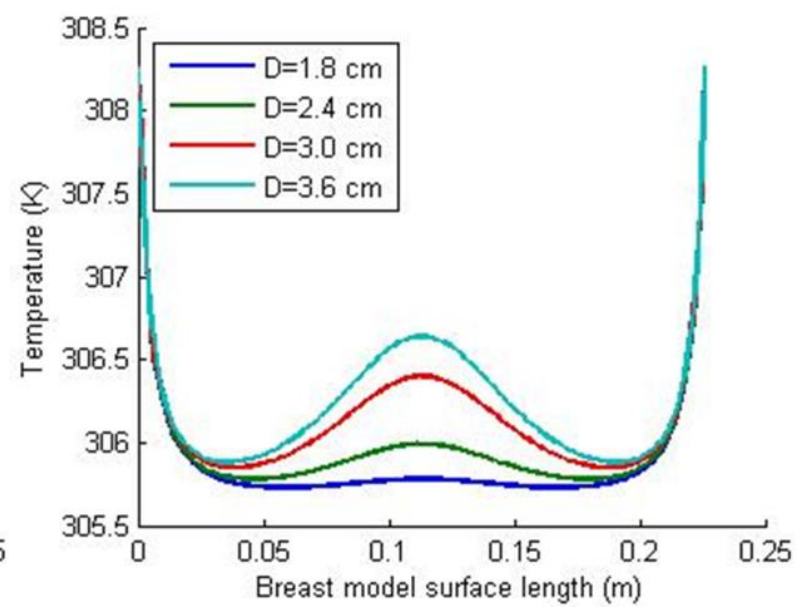

(b)

Figure 5-21 Effect of physical parameters of tumor, e.g. influence of - (a) location, and (b) diameter of tumor on breast surface temperature

environment temperature reduces the temperature elevation and area of warm zone (Figure 5-22 (b)). Therefore, a clod therapy (cold stress) or low temperature lab could provide a clear/informative thermogram.

Above analyses demonstrate that the bio-thermal parameters are a linear function of temperature and affect both the height of the temperature hill and the region on which the local warm zone (temperature hill) grows. The size of the tumour also has similar effect while the depth affects the graph non-linearly. Moreover, the influences of ambient conditions are notable and a well-designed laboratory is a vital concern for better thermogram.

Above analysis provides some general thermal feature of different parameters of breast tumour, consequently, thermal images could give some important ideas, such as confirmation of presence of a tumour, roughly the location (either deep-seated or shallow), growing rate (fast or slow growing - considering the changes in images taken in a given interval) etc. However, the interdependency between parameters needs to consider finding the exact correlation between thermal image and the tumour parameter. The parameters are interdependent in the following way:

(a) Tumor size increases with its age (growing rate) and growing rate is also related to the $Q_{m}$.

(b) Cancerous cells have higher thermal conductivity and changes in conductivity also affect the blood flow rate. 


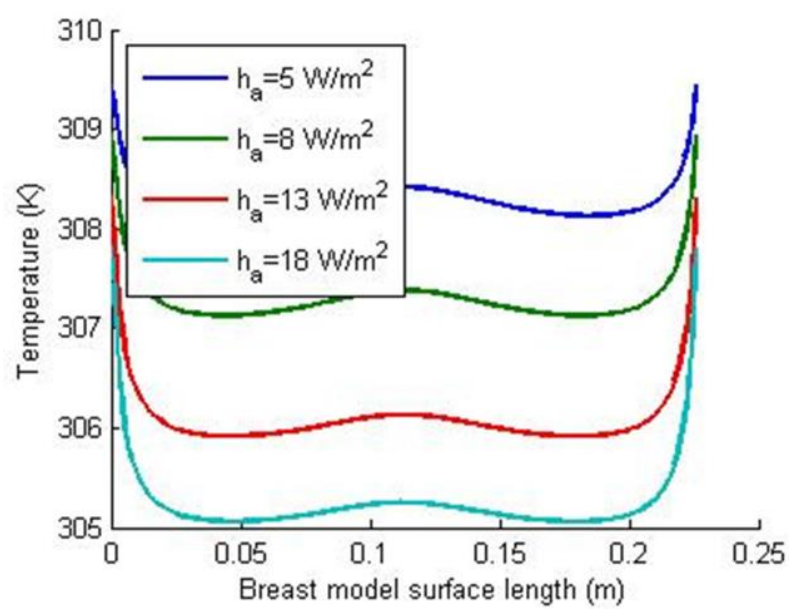

(a)

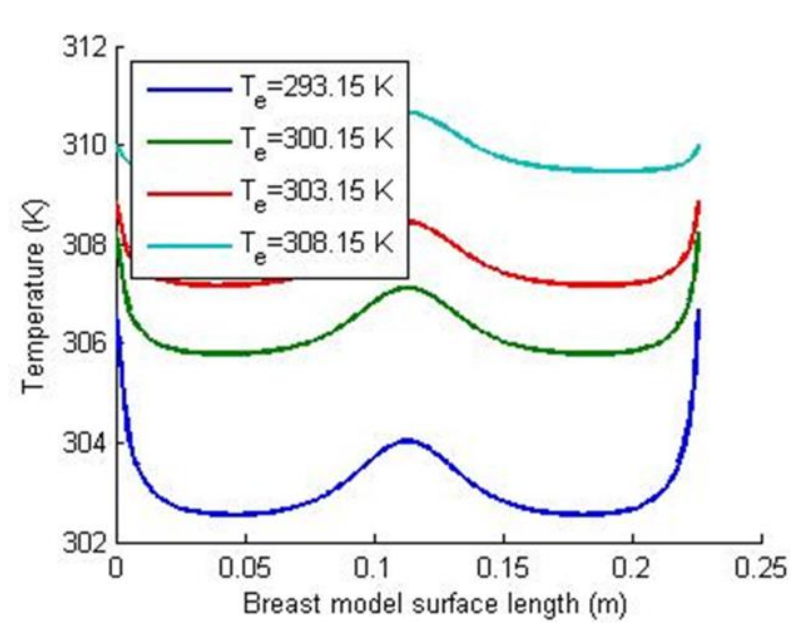

(b)

Figure 5-22 Effect of environmental parameters, e.g. influence of-(a) heat exchange rate, and (b) ambient temperature of laboratory on breast surface temperature in case of a tumor

\subsection{Deformed Breast Analysis}

Unlike any other organs in adult human, breast deformation is a common phenomenon amongst women. Drooped breast models have developed in Figure 4-11and Figure 4.12. The structural deformation might alter the thermal feature in comparing that has estimated over undeformed model. Most of the previous studies have not taken into account the deformation. This section will discuss how to calculate thermal features of deformed breast numerically with FEMwhich includes creating physical geometry, developing mesh, assigning parameter values, setting boundaries and finally computing surface temperature distribution.

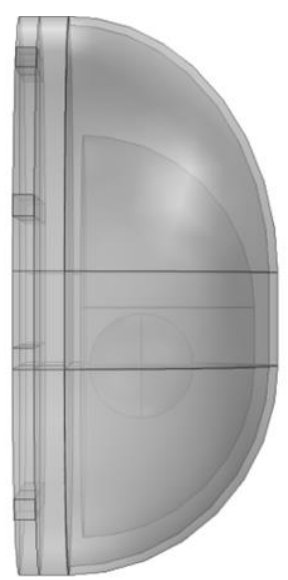

(a) Deformed breast due to body posture

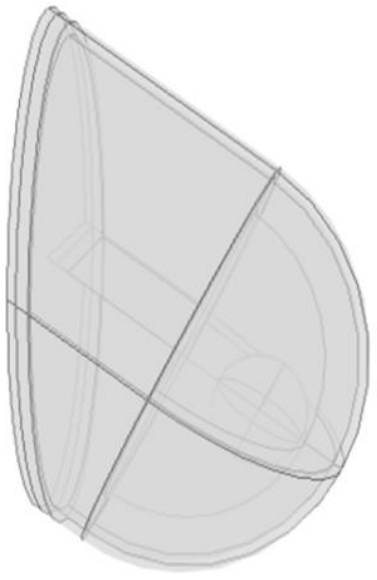

(b) Deformed (sever) breast due to ptosis

Figure 5-23 Drooped breast models 
3D FEM geometry of breast considering the drooping effect due to body posture change (standing or sitting) is shown in Figure 5-23(a). Downward nipple slip stretches the upper part, contracts the bottom part, resulting in a longer T-B line while unchanged L-R line. Such deformation leads by relative thick fat layer on top. In addition to the posture effect, the study also develop 3D FEM model, Figure 5-23 (b) for ptosis breast, in particular at 'Stage - 4'from the

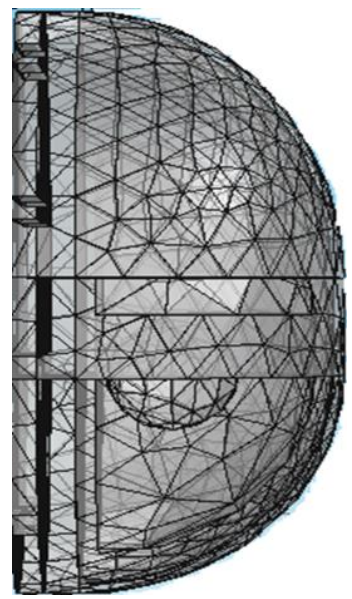

(a) FEM for Figure 5-23(a)

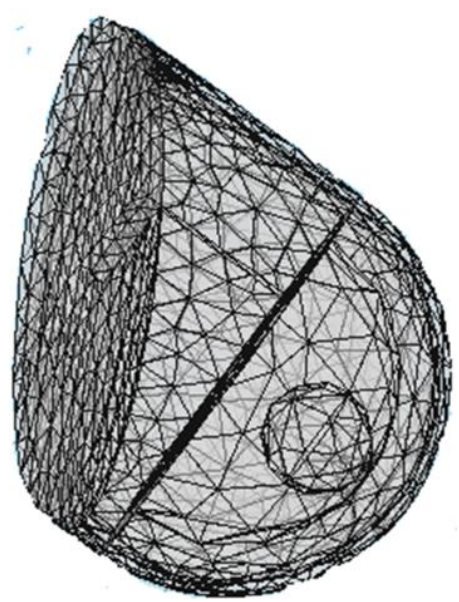

(b) FEM for Figure 5-23 (b)

Figure 5-24 FEM models for drooped breasts

geometry shown in Fig 3-12. After defining the boundaries and setting up parameters for each domain, the physical models are divided into meshes which is shown in Figure 5-24, where (a) is composed on 80000 tetrahedral and 4000 triangular elements with tumour built on 2000 tetrahedral and 200 triangular elements; and Figure 5-24(b) made on 25000 tetrahedral and 1000 triangular elements including tumour broken down upto1000 tetrahedral and 500 triangular elements.

Tumors in mild deformed breasts have been analyzed for estimating thermal distribution over the surface and along the orthogonal L-R and B-T lines over the nipple. The analyses have been performed for-Tumor 1 , malignant $D=36 \mathrm{~mm}$ located at ( $0,0,0.045), Q_{m}=80 \mathrm{KW} / \mathrm{m}^{3}$; Tumor 2, deep-seated large benign $D=20 \mathrm{~mm}$ located at $(0,0,0.04), Q_{m}=25 \mathrm{KW} / \mathrm{m}^{3}$; Tumor 3 , off-axis malignant $D=50 \mathrm{~mm}$ located at $(-0.015,0,0.033), Q_{m}=60 \mathrm{KW} / \mathrm{m}^{3}$; Tumor-4, offaxis malignant $D=36 \mathrm{~mm}$ located at $(0.015,0,0.033), Q_{m}=40 \mathrm{KW} / \mathrm{m}^{3}$. Assumed breast model is centered at origin and perfusion rate of tumors is $\omega_{b}=5.2 \times 10^{-3} 1 /$ s. Figure $5-25$ presents the 2D spatial temperature over surface for each case respectively in Figure 5-25(a) and (b) where on-axis tumors develops a pattern that is not symmetrical that has found in case of un-deformed breast. The elastic deformation causes an alteration to the thermal image which is crucial for benign 
such as Tumor-2. However, breast malignancy could restore the symmetry. In case of off-axis tumors, it is obvious that the warmest zone shifts toward the tumor center as well as losses its symmetry. Again, the malignant (Tumor 4) produces almost symmetric image around the center of the warmer region. Interestingly, both Tumor- 2 and 4 move the warm zone upward, even though the former one is grown right under the nipple which could lead to a misread location.

For the above mentioned tumors the $1 \mathrm{D}$ temperature plot along the $\mathrm{L}-\mathrm{R}$ and B-T lines are presented in Figure 5-26, where (a) presents the plot along the left-to-right horizontal line that remains unaffected due to the gravitational force and (b) presented the graph along bottom-to-top vertical line that encounters stress due to breast mass. As a result the B-T line is larger than the L$\mathrm{R}$ line by an amount around $2 \mathrm{~cm}$. The window (a) justifies that the temperature plots for above cases do not affect the horizontal symmetry. But deformation hampers the vertical symmetry even for an on axis tumor which is clearly understood from the bottom window (b). 


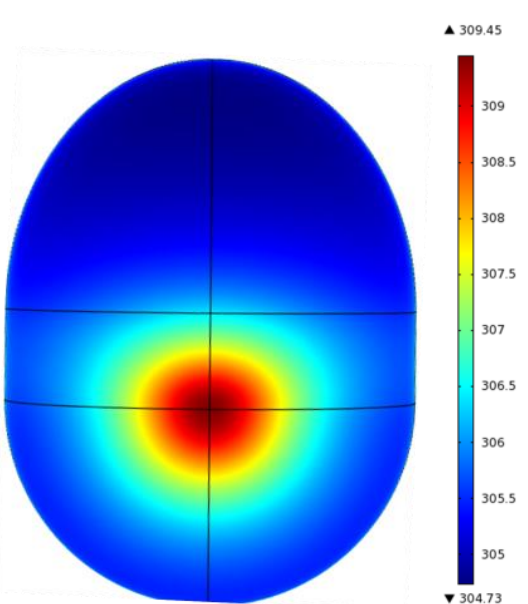

(a)

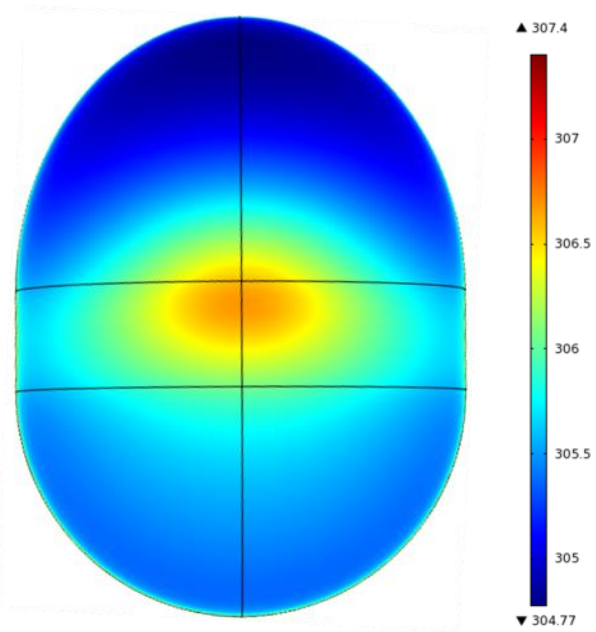

(c)

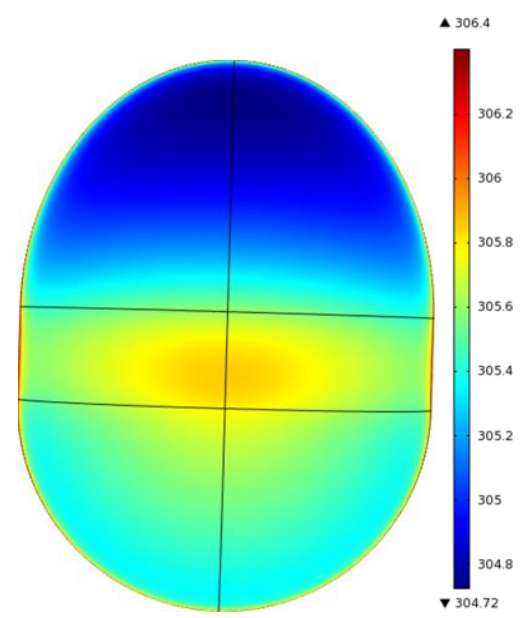

(b)

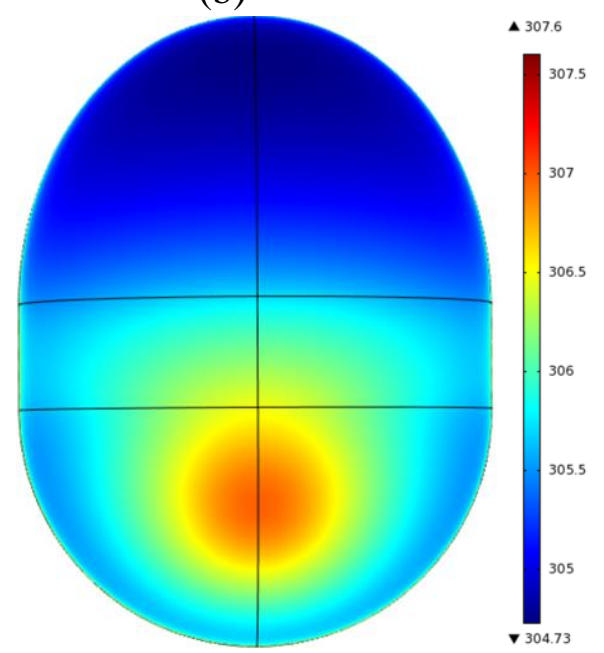

(d)

Figure 5-25 Mild deformed breast tumor thermal analyses-(a) to (f) for tumor 1 to 6 , respectively 
Tumors in ptosis stage -4 breast have been analyzed for- Tumor 1 , large benign $D=50$ mm located at $(0.051,0.078,0), Q_{m}=45 \mathrm{KW} / \mathrm{m}^{3}$; Tumor 2 , large malignant $D=50 \mathrm{~mm}$ located at $(0.051,0.078,0), Q_{m}=90 \mathrm{KW} / \mathrm{m}^{3}$; Tumor 3 , large malignant $D=24 \mathrm{~mm}$ located at $(0.051$, 0.078, 0.02), $Q_{m}=90 \mathrm{KW} / \mathrm{m}^{3}$; Tumor-4, similar to Tumor 3 located at $(0.051,0.078,-0.02)$.

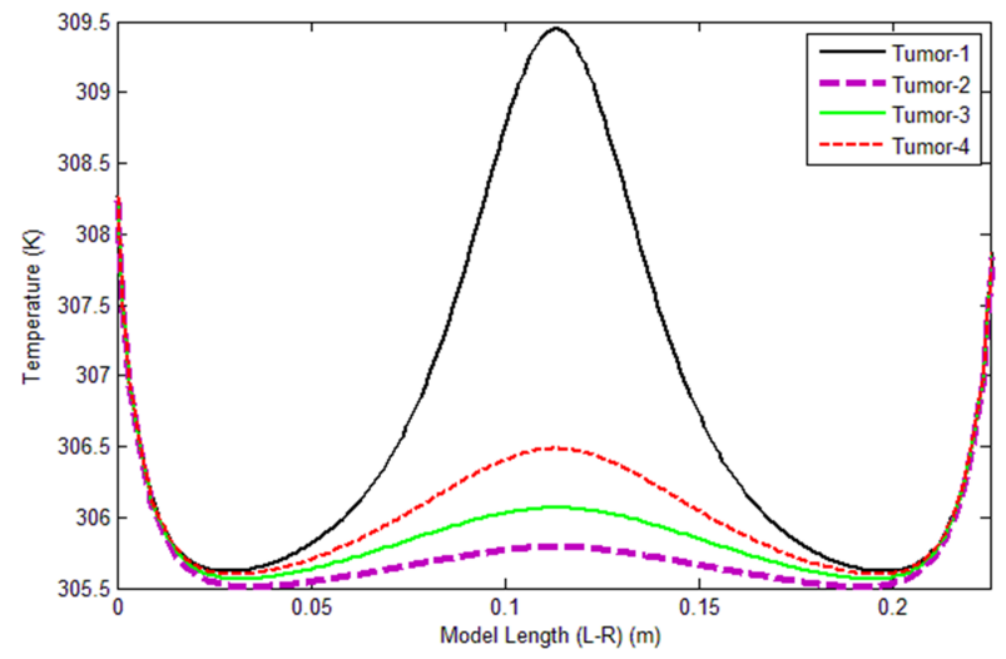

(a)

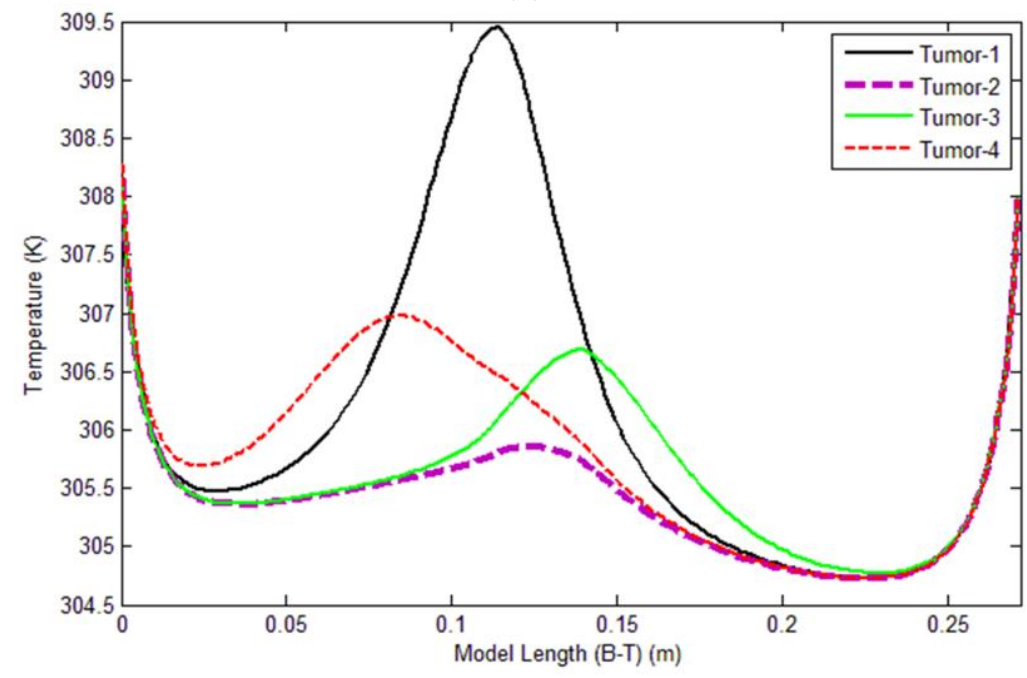

(b)

Figure 5-26 Center-line temperature for mild deformed breast tumor along-(a) L-R (left to right side) line, and (b) B-T (bottom to top) line

Assuming, at standing body posture, nipple is aligned with lower base and placed at coordinate origin. Figure 5-27(a)-(d) show the 2D temperature distribution for above four cases, respectively and (d) presents 1D temperature plots along L-R and B-T lines. Tumor 1 produce maximum almost 
$1 \mathrm{~K}$ and Tumor 2 produces $0.38 \mathrm{~K}$ at the nipple location. It is seen that due to deformation the patter losses symmetry.

The above analysis discovers that local surface temperature distribution is disturbed by the underlying tumor's bio-physical parameters and those directly affect the temperature raise, warm zone area and coldest region temperature. Model physics affects the symmetry of the pattern. Symmetry in temperature pattern is a very important feature for developing optimization dataset. 


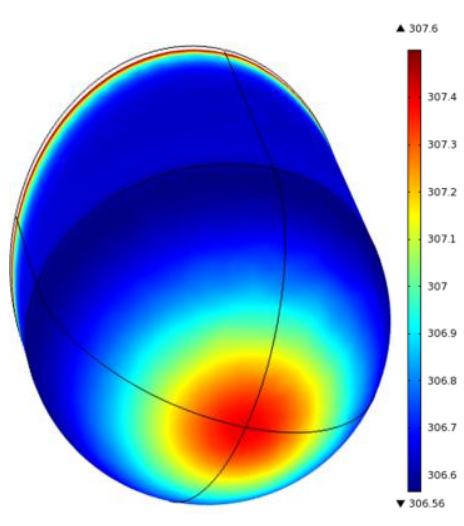

(a)

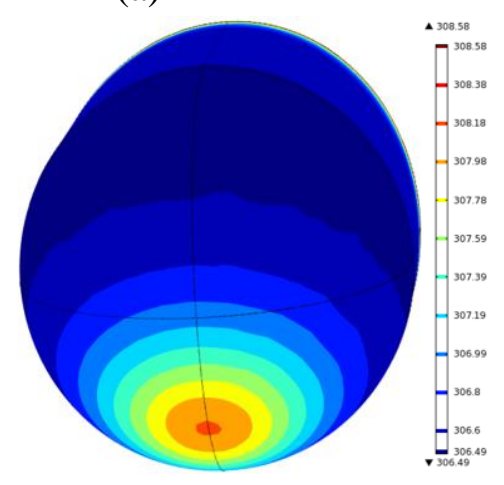

(c)

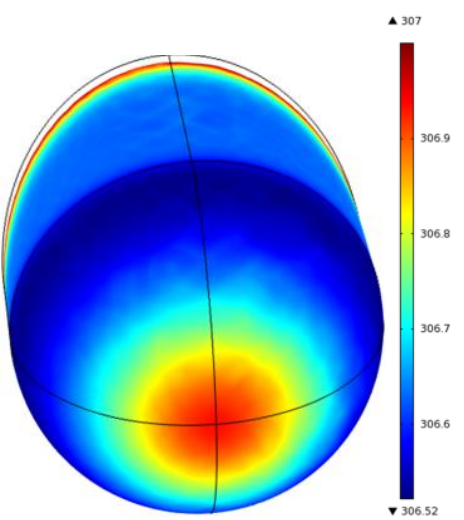

(b)

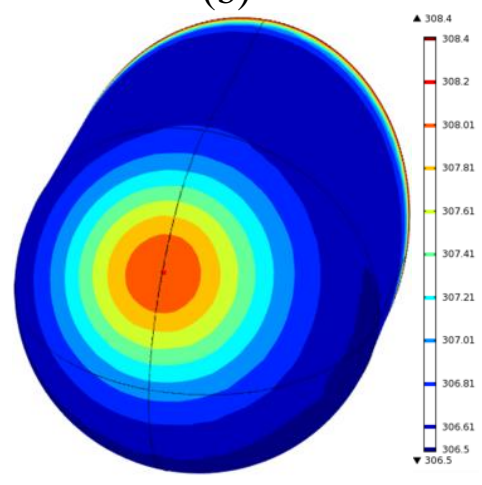

(d)

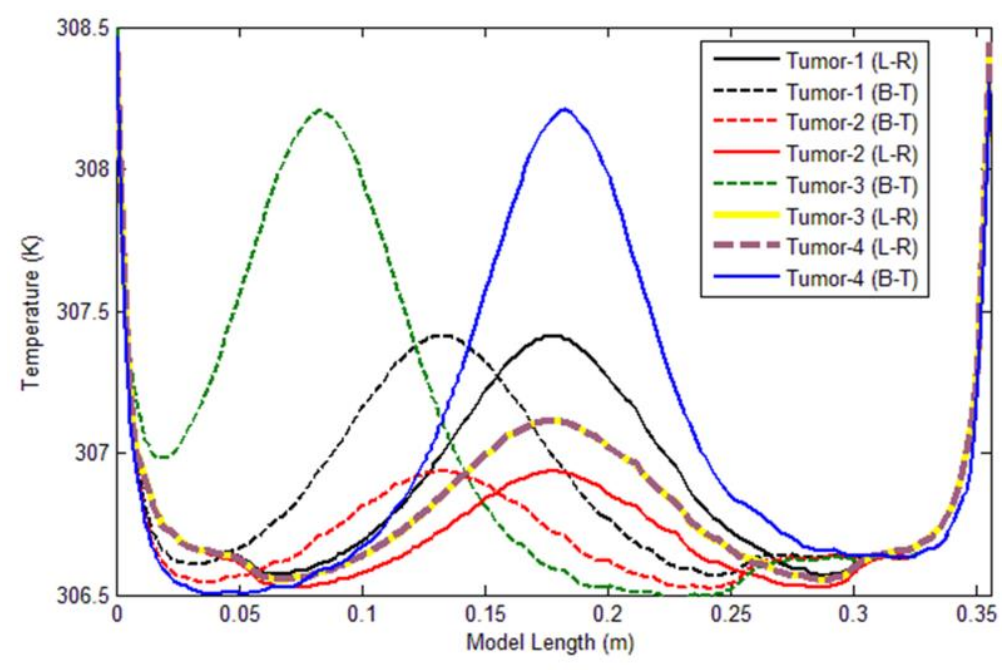

(f)

Figure 5-27 Tumor in deformed breast, (a) to (d) surface temperature for tumor 1 to 4, respectively, (f) center-line temperature 


\subsection{Summary}

In this chapter numerical methods have been used to calculate the temperature distribution over the skin surface of organ that holds a tumour. The analysis showed that the FEM approach can be standalone approach for estimating thermal features in live biological tissues. The credited built-in model while applied on the simplistic and realistic models discovered that tissue layers and types have influences on thermal figures, for example, the estimates show that the simple models have assessment error $0.8 \mathrm{~K}$ and $1 \mathrm{~K}$, respectively, over the real chest and breast models and likewise, the blood vessels in forearm causes asymmetric temperature grow where the simple model exhibits symmetry. Therefore, the anatomic structure should be interpreted properly in developing physical models. Irregular thermal estimates on the realistic models in presence of tumor has also explored that the irregularity is directly related with the physio-thermo-biological parameters of tumour. Tumour's physical parameters (i.e. size and location) have significant influences on the surface temperature profiles. The non-linear dependency between size and temperature pattern shows that the wider tumour has bigger 'temperature hill' with elevated vertex while the location vs temperature shows that deeper nodule has narrower hot zone. Similarly, the thermal parameters such as the metabolic and perfusion rate affect the thermal graphs remarkably; however, the conductive heat transfer rate shows negligible effect. Moreover, laboratory environment has influence on thermogram images too; seemingly the temperature graphs remain unaffected except move on the vertical (temperature) axis if surrounding temperature goes up. To sum up, the study presented a quantitive relation between bio-physio-thermal parameter and expected temperature texture.

Circular symmetric thermal graphs can be achieved for chest tumours merely keeping the warmest zone at the middle of the model which is straightforward as long the segmental model is legitimate. Similarly circular symmetry is always observed for on-axis breast tumours. Unlike, the forearm tumours produce only vertical symmetric thermogram because of the asymmetry to the interior make-up. However, tilted (off-axis) breast tumour develops asymmetric thermal distributions as observed. The study has showed that physical deformation of breast affect the thermal image and obvious to address it while modeling. 


\section{Chapter 6 Parameter Estimation-Indirect Approach}

\subsection{Introduction}

Computations of thermal distribution over the skin surface and inside of the human chest, forearm, and breast have been discussed in the previous chapters. Models of the above mentioned organs are proposed in Chapter 3 and the bio-heat transfer problems on those models are addressed in Chapter 4 and 5, respectively, if the organs are at healthy condition and if there is tumor underlying them. The thermal computations are obtained for a given set of bio-thermal and physical parameters of organ's tissues and tumor tissues. The approach of estimating a thermal profile for a given parameter set is called direct approach, however parameterizing tumor from the surface thermal image (thermogram) involves an indirect approach. This chapter presents an estimation methodology determining thermo-biological and/or geometrical parameters of a tumor from an abnormal temperature profile over skin surface that may be acquired by infrared (IR) thermography. The indirect estimation approach requires:

a) a test (target) vector (dataset) — which can be obtained from thermogram (in-vitro) or clinic (in-vivo) or simulation (ideal),

b) a governing equation - which can be the heat transfer equation in living body or the analogous (equivalent) heat-source model equation, and

c) a cost function - which is capable to optimize the problem involving the target dataset and the simulated (computing the governing equation) dataset.

This study will use numerical simulated datasets as the test vectors where the datasets is to be added with random noises to mimic true datasets. The governing equation must entail an accurate correlation between the thermal image and interior human physiology. The relationships have been achieved from the general solutions of the bio-heat equation, discussed in the earlier chapters. Particularly, thermal analysis of homogeneous tissues in chapter 4 has discovered that the static gradient of the abnormal local skin temperature to the altered physio-thermo-biological parameters have straight-forward association. The thermal gradient (contribution of thermophysical parameters to the skin temperature), alternatively, the irregular spatial temperature distributions on local surface will be expressed as a function of diagnosis parameters and thus will replace the 'bio-heat' model with an equivalent 'heat-source' model. The characteristic expression 
(governing equation) of the analogous model will be solved and converged to predict tumor parameters, more specifically, the size, location, perfusion rate and heat rate of hyperactive region while convergence will be done by minimizing a fitness (cost) function. Derivation of the governing equation and the fitness function will be detailed in this chapter.

Thermal behaviours of anatomic-accurate models, shown in chapter 5, are quite different from that observed in simplified models. Consequently, parameterization of heat flow graphs in realistic models is complicated, following on a labours job to find analogous heat-source model. In such case the bio-heat equation will be employed as the governing equation. This chapter will utilize the gradient projection method (GPM) for estimating heat-source parameter while the homogenous tissue will be modelled as a heat source. In addition, the pattern search method (PSM), genetic algorithms (GA) and artificial neural network (ANN) methods will be used in an invasive diagnosis of tumor in heterogeneous tissues.

Essentially, all optimization approaches require an initial parametric matrix which can be chosen from the heat-source model using approximation technique or from clinical inspection or from a random function generator. After selecting the initial matrix, the GPM will move to the next solution with predefined incremental matrix where the increment factors are determined sensing the static temperature gradient. Perturb (with random initial solution) and solve the GPM repeatedly unless the solution matrix comes up with a set of possible solutions (local and/or the global minima) from which the best solution (global minima) is sorted out using physical constraints and clinical knowledge. Unlike GPM, the PSM moves on a particular path (search direction) defined by 'directional matrix' and converges with adaptive step sizes. Exposing the thermal directional derivatives, while calculating the step sizes and searching for in every directions, increase the probability of converging to the global minima (best result). However, the process increases the computation time as well as the memory usage. Unlike GPM and PSM, the GA converts the solution matrix into binary digit to form chromosomes while the bit(s) are swapped inter-chromosomes or intra-chromosome to move on the next possible solution. An NP hard, non-gradient type optimization approach needs GA to solve several times to come up with unique result. This study will run the GA thrice with different random initial populations, however, the final estimates will be found identical regardless the initial population. 
The objective of this chapter is to develop a methodology to estimate the size, depth and heat generation rate of an embedded tumor in the human body organ, based on the temperature profile on the surface that may be obtained by thermogram (in-vitro) or clinic (in-vivo) or simulation (ideal). This study will use numerical simulated data enveloped with up to $\pm 10 \%$ random noise as the test vector (target dataset) in lieu of thermogram. The heat-source model equation will be applied to produce the simulated dataset for the cost function in the GPM optimization while the other methods will use the bio-heat transfer equations. The bio-heat equation will be solved numerically with the FEM solver. The approximate values to the parameters will have been justified and fairly accurate values will be, then, applied to form the initial solution matrix for optimizing the bio-heat equations. Formulation for the cost function will be discussed, in detail, in this chapter.

Indirect solving with the PSM was articulated by Agnelli et al [14] where the authors had implemented a FDM scheme to solve bio-heat problems on a cuboid domain (rectangular box tissues) with mixed mode boundary conditions and then, the optimization algorithm (PSM) was used estimating the hyperactive region (spherical tumor) parameters by minimizing a fitness function involving the temperature profiles obtained from the FDM and test dataset. The laboratory dataset was added with noise to produce the test matrix. In-vitro experiment on a cylindrical adipose model, heated up with a resistive heater was applied to predict the heat source parameters (location and heat rate) with a GA based inverse approach in [60]. Estimation of breast tumor thermo-physical parameters using ANN and GA was discussed in [16] where the ANN was used to map the relationship of tumor parameters (depth, size and heat generation) to the temperature profile over the idealized breast model, the relationship obtained from ANN is compared with numerical result and finally GA was used to estimate the parameters by minimizing a fitness function involving comparing the temperature profile from simulated (or clinical) data to those obtained by ANN.

Certainly, the accurateness of the estimated parameters depends on the performance of the cost function which involves a target dataset and a simulated dataset. As discussed, the target dataset was determined from the FDM scheme enveloped with noise $(\leq \pm 10 \%)$ and simulated dataset was obtained by solving bio-heat problem (using the FDM) on a two-domain (healthy and tumor domain) cuboid tissue model in [14]. Therefore, the weaknesses of the study are: (i) the geometric model of organ is simplistic, therefore, the thermal datasets such as the test and 
simulated datasets are blurred, (ii) the approach needs to modify extensively before applying to any human organ, and (iii) the values of some bio-thermal parameters, especially the perfusion coefficient, are not supported by any source. The restrictions of [60] are-(i) target dataset was obtained from the cylindrical adipose surface which was heated up with a resistive heating system, but the relation between the heater and tumor was completely unknown, (ii) the perfusion heat loss was neglected, and (iii) the numeric scheme had used to compute the simulated dataset was unclear. However, the necessity of the anatomic accurate model and numeric datasets are excluded in [16] with the help of the ANN and GA but the sources of thermal datasets were not disclosed.

\subsection{Introduction to Optimization}

Optimization is an important tool that analyzes a physical system and makes decisions for finding the best solution from the set of all feasible solutions. The process, basically, involves minimizing a cost function which includes target and simulated datasets. Not to mention the technique of generating the target datasets, the basic optimization process includes constructing a model, determining the problem type and selecting the solution approach. Modeling is the process of identifying and expressing the objective (quantitative measure of system performance, for example, deviation minimization between observed and predicted data), the variables (parameters of the model), and the constraints (relationships among the variables and their allowable limits) mathematically. Tailoring an appropriate optimization algorithm to a particular type problem is the second preference. The model performance depends on the accurateness of the optimization algorithm that depends on the nature of the variables (model parameters) and comprehending the problem type. For instance, the variable can be continuous or desecrate, unconstraint or constraint; and the problem can have none, one or many objectives and can be deterministic or stochastic. Finally, the algorithm will be coded to solve the thermal problem with computers. The details about modeling, cost function, variables, constraints, optimization algorithms have been discussed in following sections.

\subsubsection{Diagnosis Parameters and Modeling}

Analysis discovered that abnormal temperature profile over a faulty organ is a function of heat conductivity $(k)$, metabolism $\left(Q_{m}\right)$, perfusion $\operatorname{loss}\left(\omega_{b}\right)$, tumor location $(d)$ and $\operatorname{size}(R)$, ambient temperature $\left(T_{e}\right)$ and heat exchange rate $\left(h_{a}\right)$. This chapter is dedicated to estimating some characteristic parameters that might help in detecting and localizing tumors. Ambient effect 
$\left(T_{e}\right.$ and $\left.h_{a}\right)$ can be excluded from the above list with designing a perfect matched laboratorysimulation environment. Therefore the parametrization process includes first five parameters mentioned above. A customized optimization algorithm is designed for minimizing the temperature function where the function may be achieved from numerical solution of bio-heat transfer problem or derived from a governing equation with replacing the bio-heat phenomena to a mathematical model. Generally, modeling is the process of representing a real-world object or phenomenon as a set of mathematical equations. Derivation of the model for living bio-heat transfer phenomenon is going to be discussed following. It is worth mentioning that the patient and thermogram technologist are not accustomed with some bio-thermal parameters such as $k, Q_{m}$ and $\omega_{b}$, therefore, in the proposed model will replace them into a generally sensible parameter.

Diagnostic variable set includes the depth, size and body temperature of the hot nodule will be estimated. Extracting these parameters from in-vivo bio-thermal feature requires transformation of bio-thermo-physical variables $\left(k, Q_{m}, \omega_{b}, d, R, T_{e}, h_{a}\right)$ into diagnostic variables $\left(d, R, T_{c}\right)$. Such conversion evokes a process called "domain transformation" which is presented in Figure 61 where the left circle indicates the bio-thermo-physical domain, the right circle is the diagnostic parameter domain and the surrounding rectangle is the coupling media, the ambient. The figure signifies that the Pennes bio-heat parameters can be turned into underlying fault indicator parameters if the process is done in a specific well controlled ambient. Both domains share two common parameters the conduction $k$ and artier temperature $T_{a}$. The domain transformation follows Eqn. 6.1.

$$
T \equiv f\left(Q_{m}, \omega_{b}, c_{b}, d\right) \underset{k, T_{a}, h_{a}, T_{e}}{\rightleftarrows} T \equiv f\left(d, T_{c}, R\right)
$$


The auspicious transformation has come up with a heat source model, the formation of such model is discussed in the next section.

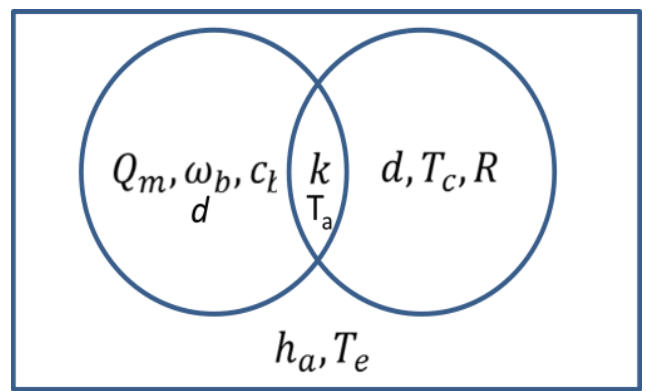

Figure 6-1 Bio-heat and diagnosis domain

\subsubsection{Governing Equation}

The equation or set of equations are to be solved with a suggested solution given by an optimization algorithm are called the governing equation(s). The bio-heat equation arameters shown Figure 6-2 that mimics very similar results to those observed in Pennes' model. Bio-thermo-

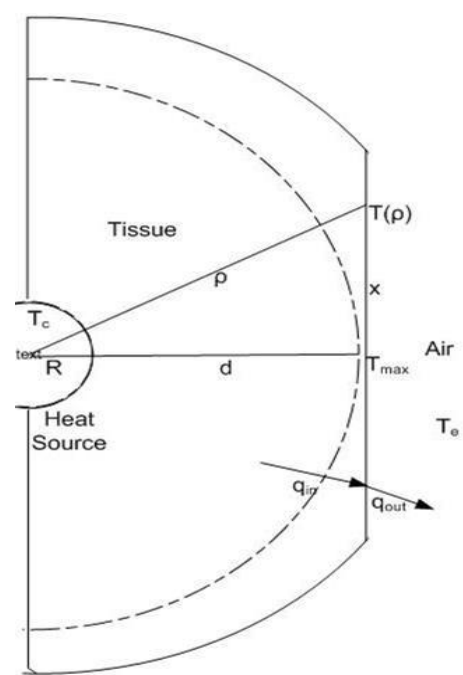

Figure 6-2 Heat source model

physical characteristics of a homogeneous, isotropic and temperature invariant healthy organ are converted to the model parameters where the healthy tissues are replaced with a pinhead heater of negligible radius $(\mathrm{R})$, located at $d$ (same to the tissue thickness) and maintain a constant temperature, $T_{c}=$ artier temperature, $310.15 \mathrm{~K}$. However, in case of tumor, the hyperactive nodule 
will be replaced by a spherical heat source of uniform body temperature $T_{c}>310.15 K$ and radius $R$. In comparing to the dimensions of surrounding tissues and using the boundary conditions in Eqn. 3.4-b and with the help of [57], the temperature distribution of the model is calculated as:

$$
T(\rho)=T_{e}+\frac{T_{c}-T_{e}}{\frac{\gamma h_{a}}{k R} \rho(\rho-R)+1}
$$

where $T$ estimates interior temperature if $\rho<d$ and surface temperature if $\rho \geq d$ and $\gamma$ a non-linear parameter, termed as model constant, to be determined by inspection. On model surface $\rho=\sqrt{d^{2}+a^{2}},(\rho \geq d)$ and $a$ is the distance of a point on surface from the $T(d)$ location. The temperature at $\rho=d$ where $d$ is the depth of the tumor is the highest temperature on the model surface; $T(\rho=d)=T_{\max }$. Temperature at any arbitrary point on model surface is given in Eqn. 6.3 .

$$
T(a)=T_{e}+\frac{T_{c}-T_{e}}{\frac{\gamma h_{a}}{k R}\left(\sqrt{d^{2}+a^{2}}\right)\left(\sqrt{d^{2}+a^{2}}-R\right)+1}
$$

Eqn. 6.3 requires proper evaluation of the model constant $\gamma$ which is done matching the response with thermal computation observed in bio-thermo-physical domain. The following nonlinear expression of $\gamma$ is determined that provides the best performance of the model with almost $98 \%$ accuracy $\left(\%\right.$ accuracy $\left.=\frac{1}{N} \sum_{i}^{N}\left|\frac{T_{\text {sim }}-T_{\text {model }}}{T_{\text {sim }}}\right|_{i}\right)$ :

$$
\gamma=2000\left((d-2.2)^{2}+2.6\right)(R n)^{-0.92}
$$

The constant $\gamma$ has empirical relationship with hot nodule location, size and heat-rate intensify factor $n$, which is defined as a ratio of heat rates of hot nodule to healthy tissue's natural metabolism, which can be written as $n=\frac{Q_{m_{\_} t u m o r}}{Q_{m_{-} \text {healthy }}}$. The factor $n$ is also a function of $R, T_{c}$ and $d$ which is determined as,

$$
n=\frac{1}{6} \times\left[10^{4}\left(\log \frac{T_{c}-273.15}{-0.111 d^{2}+0.64 d+34}\right)-33.4 R\right]
$$

Eqn. 6.5 can be rewritten as,

$$
T_{c}=\left(-0.111 d^{2}+0.64 d+34\right) e^{10^{-4}(6 n+33.4 R)}-273.15
$$


(a)

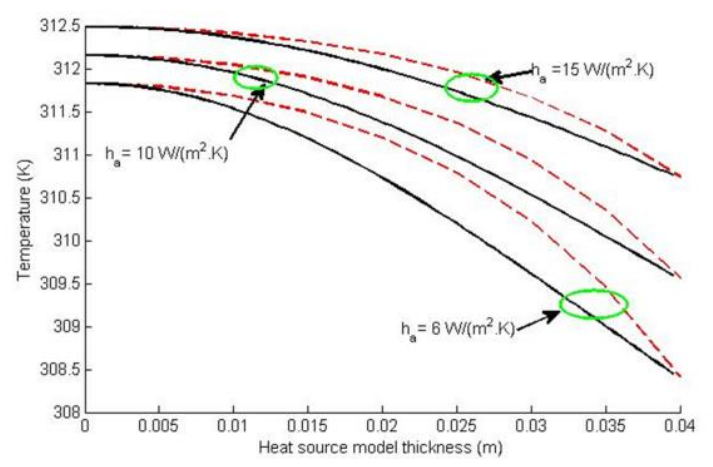

(b)

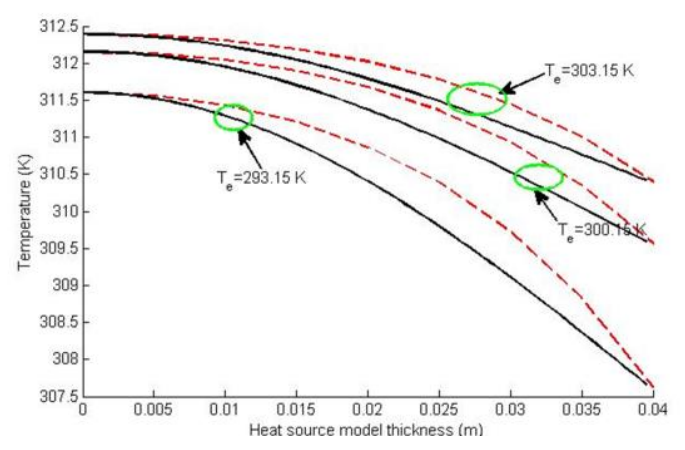

(c)

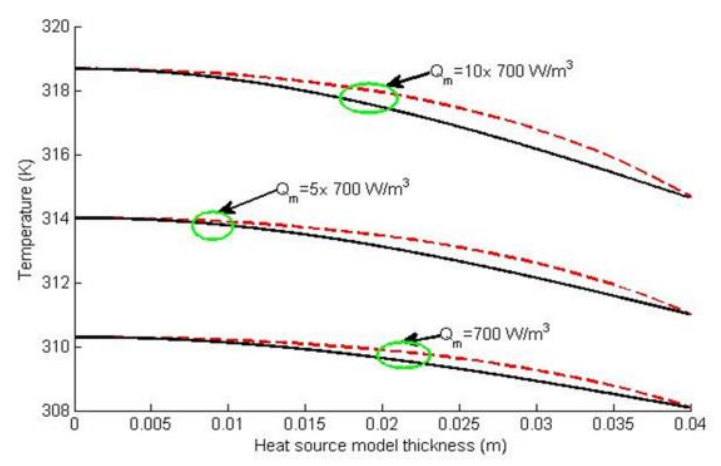

(d)

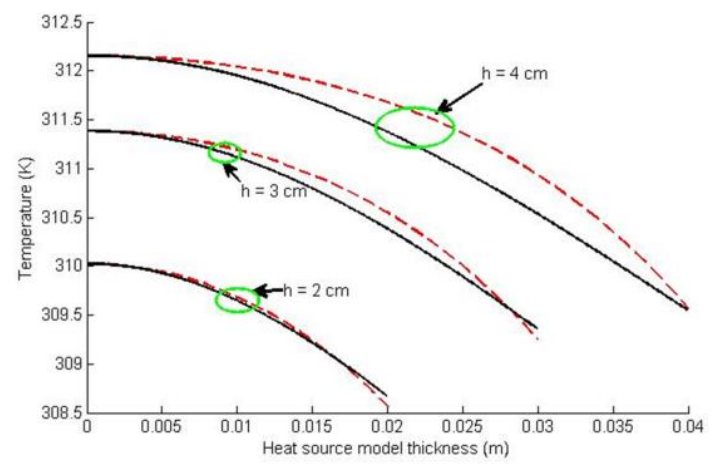

Figure 6-3 Comparison of heat source model response with analytic result for various - (a) heat exchange rate, (b) ambient temperature, (c) metabolic heat and (d) thickness 
To summarize, Eqn. 6.4 calculates the model coefficient which is a function of heat source parameters, Eqn. 6.5 gives the ratio between metabolic rates of tumor and healthy tissues which can also be computed from model parameters, and Eqn. 6.6 shows how the model parameters contribute to the tumor bulk temperature. For respective cases, through Eqn. 6.4 to Eqn. 6.6, the $d$ 's are in $\mathrm{cm}$ and $R$ 's are in $\mathrm{mm}$.

The proposed model requires validation to investigate how the model performs. For this, 1D radial temperature flows in model inside and in tissue interior are investigated solving Eqn. 6.2 (diagnosis domain) and Eqn. 4.26 (bio-thermo-physical domain), respectively, separately and their responses with variable heat exchange rates, ambient temperatures, heat generation rates and tissue depths. Temperature distributions estimated on both domains are presented in Figure 6-3, where the responses of the bio-heat model are depicted in 'dotted' and those of the heat-source model are in 'solid' lines. The effect of heat loss rates at skin surface is presented in Figure 6-3(a), which shows that the model performance improves at higher exchange rate as observed on the overlapped profiles. However, the heat source model estimates fairly accurate temperature at boundaries which plays a very vital role in determining surface temperature. A similar effect is discovered in (b) where the responses of the models with different environment temperatures are examined. The influence of the metabolic heat rate on the responses of the models, as shown in Figure 6-3(c), is quite opposite where the discrepancy increases if the metabolism increases. The models' responses for different tissue thicknesses (i.e. heat source depth) are showed in Figure 6-3(d); it is evident that the model responses may lose consistency for deep-seated tumors. However, the temperature graphs show that the proposed heat source model can provide an approximate result to the bioheat model. Consequently, the heat source model could provide solutions closer to the accurate values while optimized. In clinic application, though, the exact parameters are always desirable which can be found from the bio-heat model, however solving detailed bio-heat models using finite element method is very time consuming during optimization process with a random initial solution vector. Therefore, a compatible tool, the heat-source model, has been recommended. The major advantage of the model is that thermal computation does not require FEM, leading to reduced computational time and memory usage.

Another advantage is that, it can provide the temperature plots both in tissue inside and over surface, therefore, the model may be used for tumor ablution as well. 
The model is developed for homogeneous, isotropic and cuboid tissues, indeed a major lacking which, consequently, might lead to a pessimistic estimate. In addition, consistent estimated temperature profile does always not grantee consistency for a wide range of variations.

Despite this observation, this study will apply the proposed model to estimate the approximate solution matrix and the solutions are given as a potential solution to the finite element analysis to predict the best solution and thus reduce the computation time as well as ensure rapid convergence. Computing the value of temperature $T_{e s t}=f\left(R, d, T_{c}\right)$ by systematically choosing input values and minimizing the expression $\min \left\|T_{t h}-T_{e s t}\left(R, d, T_{c}\right)\right\| \leq \delta$ (cost function or objective function), there exists some $\delta>0$, where $T_{t h}$ is acquired from thermogram image.

\subsubsection{Variable's Characterization and Initialization}

Abnormal bio-thermal behaviour of tumor can be investigated by physical exam (biopsy), imaging, endoscopy, and lab tests whether only the biopsy can usually tell the type, stage, size and shape. It is proven from the biopsy sighting that a tumor or a lesion or a mass can grow on any tissue layers such as on bone tissues, on soft tissues, on adipose tissues, on skin tissues which confirms that the diagnosis parameters or, alternatively, the heat-source model variables can likely be constrained.

Starting with an initial vector $X_{0}\left(d, R, T_{c}\right) \in \Omega$ the optimization process will search for valid solutions. The initial solution vector $\left(X_{0}\right)$ plays an important role in fast converging and/or finding local/global minima. Taking the lowest possible values for $R_{0}$ and $T_{c_{0}}$, the calculation for $d_{0}$ follows as:

Rewrite Eqn. 6.2 for $\rho>d$ and $\rho=d$, respectively.

$$
T(\rho)-T_{e}=\frac{T_{c}-T_{e}}{\frac{\gamma h_{a}}{k R} \rho(\rho-R)+1}
$$

and

$$
T_{\max }-T_{e}=\frac{T_{c}-T_{e}}{\frac{\gamma h_{a}}{k R} d(d-R)+1}
$$

Since $R \ll d$, hence neglecting the effect of $R$ and dividing Eqn. 6.7 by Eqn. 6.8: 


$$
\frac{T(\rho)-T_{e}}{T_{\max }-T_{e}}=\frac{\frac{\gamma h_{a}}{k R} d^{2}+1}{\frac{\gamma h_{a}}{k R} \rho^{2}+1}
$$

Replacing the equation with surface temperature and rewriting,

$$
\begin{aligned}
& \frac{T(a)-T_{e}}{T_{\text {max }}-T_{e}}=\frac{\frac{\gamma h_{a}}{k R} d^{2}+1}{\frac{\gamma h_{a}}{k R}\left(d^{2}+a^{2}\right)+1} \\
& \frac{T(a)-T_{e}}{T_{\max }-T(a)}=\frac{\frac{\gamma h_{a}}{k R} d^{2}+1}{\frac{\gamma h_{a}}{k R} a^{2}+1} \\
& d=\sqrt{\frac{T(a)-T_{e}}{T_{\max }-T(a)} a^{2}-\frac{k R}{\gamma h_{a}}} \\
& d_{0} \cong \sqrt{\frac{T(a)-T_{e}}{T_{\max }-T(a)}} a
\end{aligned}
$$

Therefore the initial solution vector $X_{0}\left(d_{0}, R_{0}, T_{c 0}\right)$ is modified repeatedly within the solution space and the optimization process advances.

\subsubsection{Cost/Objective Function}

An optimization problem consists of maximizing or minimizing a real function by systematically choosing input values from within an allowed set and computing the value of the function. More generally, optimization includes finding the "best available" values of some objective function given a defined domain (or a set of constraints), including a variety of different types of objective functions and different types of domains. In this study two objective functions have been proposed, as follows:

$$
\begin{aligned}
& O F_{1}=\sum\left\|T_{I R}(\Omega, a)-T_{\text {sim }}(\Omega, a)\right\|_{\min } \\
& O F_{2}=\sum_{T_{\text {const }}}\left\|r_{I R}(\Omega)-r_{\text {sim }}(\Omega)\right\|_{\text {min }}
\end{aligned}
$$

where $\Omega$ is the diagnosis vector, $\Omega \in\left\{d, R, T_{c}\right\}$; $a$ is the space vector a $\in\{x, y, z\}$ and the suffixes $I R$ and $\operatorname{sim}$ are test data and simulated data, respectively. In $O F_{1}$ the cost function minimizes the average of absolute difference between spatial temperature data that is estimated over the heat source model to that is captured by infrared thermography. On the other hand, the function $O F_{2}$ 
minimizes the average of absolute difference between distances of isothermal contour from the warmest point of the estimated and the IR captured image. Target temperature dataset for $O F_{1}$ is prepared from thermogram image by choosing random points and reading the temperature at those points where the random points may be chosen systematically (equally spaced) or arbitrarily. The study has chosen arbitrary temperature samples with random function generator algorithm. The thermogram is divided into some isothermal contour using modified snake algorithm and then again divide using equal-spacing spider web algorithm; the location of the intersection points of isothermal contours and radial lines formed using the above mentioned algorithms, respectively are to form a matrix called spatial-location dataset. This dataset is used in the second objective function.

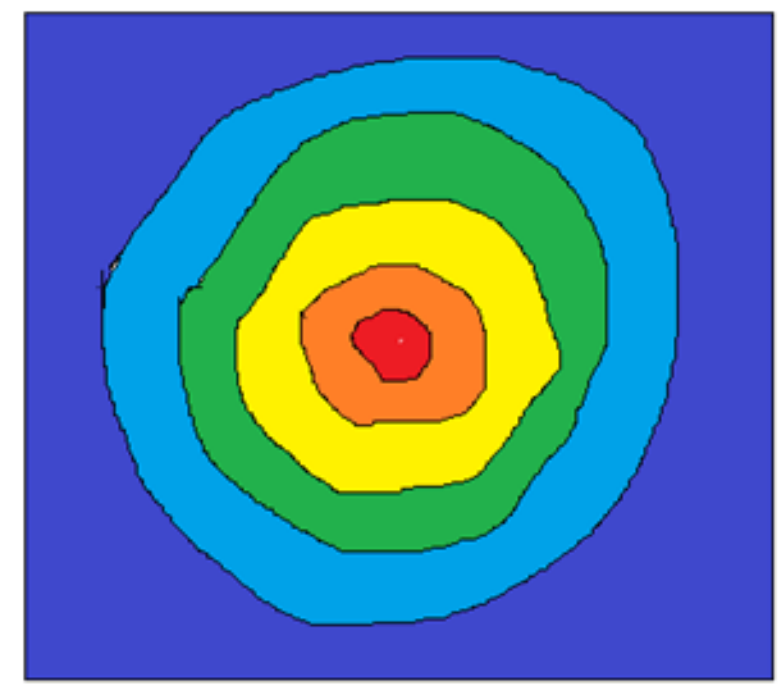

Figure 6-4 A sample thermogram

Generation of datasets for the above objective functions from a typical temperature distribution shown in Figure 6-4 is the following. Identify the highest and lowest temperatures to calculate the temperature elevation $\Delta T$ and determine the number of isothermal contours $n=\frac{\Delta T}{0.02}$, therefore the isothermal temperatures are $T_{i}=T_{\min }+m \frac{\Delta T}{0.02}$, where $m=0,1,2, \ldots \ldots, n$, respectively from the coldest contour. Using modified snake algorithm, divide the image and produce $n$ lines of constant $T_{i}$ as shown in Figure 6-5. The figure also shows the random locations of at least 200 points and temperature of each point and their respective locations are stored in a matrix to produce dataset for $O F_{1}$. Further, the thermal image is divided by 28 equally spaced lines using spider web algorithm, the locations of the intersection points between the isothermal and 
these radial lines, as shown in Figure 6-6, are captured and stored on a matrix that produce the dataset for $O F_{2}$.

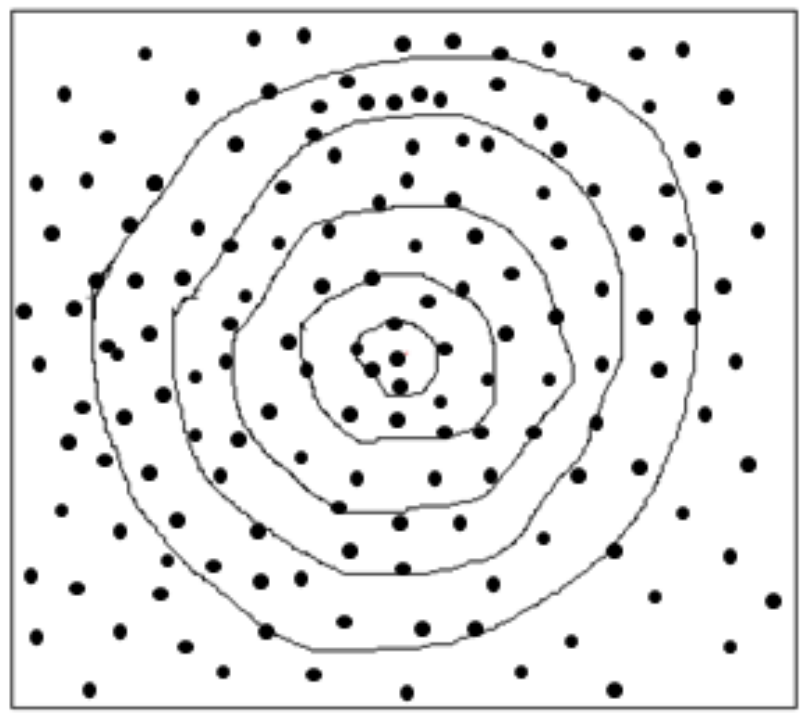

Figure 6-5 Distribution of random data points

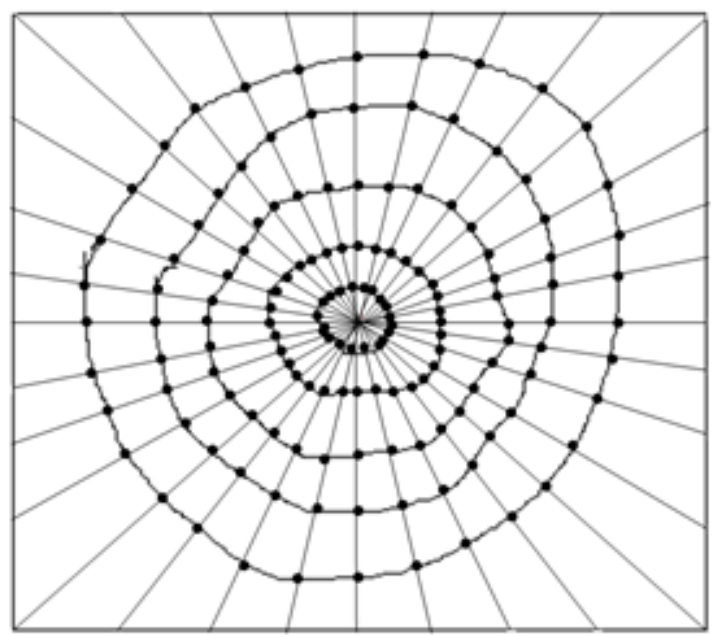

Figure 6-6 Equal spaced isothermal points

\subsubsection{Problem Type}

Before tailoring an optimization algorithm for a problem, the problem should be investigated properly so that the algorithm fits well with the features of the particular system. The data (temperature) fitting problem of the research has the following character: 
- Continuous: model has continuous variables, therefore, at a point $X \in \Omega$ can deduce information at the neighborhood of $X$ which would help determining adaptive step sizes $(\nabla)$.

- Constrained: there are explicit constraints on the variables which limit the solution domain. Moreover, interdependency of the variable makes the optimization process complex.

- Multiple Objectives: the problem has two objective functions.

- Stochastic: the parameters are known with certain bounds, the goal is to find some policy that is feasible for all (or almost all) the possible data instances and optimizes the expected performance of the model.

\subsubsection{Optimization Problem Solver}

Many optimization solvers are available commercially solving typical inverse problems. However developing customize optimization algorithm is a general practice. The study will tailor customize source code under MATLAB for indirect solution of bio-thermal problem in the presence of underlying tumors. The following sections discuss the pros and cons of several approaches.

\subsection{Optimization Approaches}

Optimization algorithms have been designed to minimize the above objective functions with the criteria discussed in above. Typically, in the presence of the set of bound and more general constraints, the decision variables are optimized. This stochastic problem is solved in Gradient Projection Method (GPM), Pattern Search Method (PSM), Genetic Algorithm (GA) Method and Artificial Neural Network (ANN) method. The optimization method works in the following way:

$$
\min f(x)
$$

subject to $x \in \Omega$

where, $x$ is a vector $x^{T}=\left[x_{1}, x_{2}, \ldots \ldots, x_{n}\right]$ and $\Omega:=\left\{\mathrm{x} \in \mathbb{R}^{\mathrm{n}}: l \leq \mathrm{x} \leq \mathrm{u}\right\}$, where $l, u \in$ $\mathbb{R}^{n}$ and $l:<u$. 


\subsubsection{Gradient Projection Method (GPM)}

GPM is a powerful tool for bound constrained optimization. The method is based on projecting the search direction into the subspace tangent to the active constraints. For the given constraints the projection can be expressed component-wise as:

$$
\left[P_{\Omega}(x)\right]_{i}:=\left\{\begin{array}{lc}
l_{i} & \text { if } x_{i} \leq l_{i} \\
x_{i} & \text { if } l_{i}<x_{i}<u_{i} \\
u_{i} & \text { if } x_{i} \geq u_{i}
\end{array}\right.
$$

The projection matrix $\mathbf{P}$ is related with the direction of search as

$s=-\boldsymbol{P} \nabla f=-\left[I-\boldsymbol{N}\left(\boldsymbol{N}^{T} \boldsymbol{N}\right)^{-1} \boldsymbol{N}^{T}\right] \nabla f$, where column of the matrix $\boldsymbol{N}$ are the gradients of these constraints, which is defined by $n_{i j}=\frac{\partial g_{j}}{\partial x_{i}}, i, j=1,2, \ldots \ldots, n$. (Assume all constrains are active all the time). The $\nabla f$ vector of this function is given by the partial derivatives with respect to the each of the independent variables,

$$
\nabla f(x)=\left[\begin{array}{c}
\frac{\partial f}{\partial x_{1}} \\
\frac{\partial f}{\partial x_{2}} \\
\vdots \\
\frac{\partial f}{\partial x_{n}}
\end{array}\right]
$$

The basic assumption of gradient projection method is that $x$ lies in the subspace tangent to the active constraints. If $x_{i+1}=x_{i}+\alpha s$, and both $x_{i+1}$ and $x_{i}$ satisfy the given constraints, $\alpha$ is a positive sclar. The algorithm for constrained gradient-projection optimization can be described as follows.

Let start with $k=0$ and an estimate of $x^{*}, x_{k}$.

1. Test for convergence: If the conditions for convergence are satisfied, then stop and $x_{k}$ is the solution. Else, go to Step 2.

2. Compute a search direction: Compute the vector $p_{k}$ that defines the direction in $n-$ space along search.

3. Compute the step length: Find a positive scalar, $\alpha_{k}$ such that $f\left(x_{k}+\alpha_{k} p_{k}\right)<f\left(x_{k}\right)$.

4. Update the design variables: Set $x_{k+1}=x_{k}+\alpha_{k} p_{k}, k=k+1$ and go back to 1 . (Please note that $\alpha_{k} p_{k}=\Delta x_{k}$ ) 


\subsubsection{Parameter Assortment}

The study limits diagnosis of soft tissue tumors, only assuming chest and forearm tumors are developed entirely in the muscle layer and breast tumors in the lobule layer. Thus, the radius and depth variables (parameters) abide by certain constraints. Likewise temperature at tumor center rate can also be bounded with some restrictions. The solution space $(\Omega)$ is given by:

$$
\Omega=\left\{\begin{array}{cc}
h & t-R \leq h \leq t_{\text {skin }}+R \\
R & R \leq\left(t-t_{\text {skin }}\right) / 2 \\
T_{c} & T_{c} \leq 313.15^{0}
\end{array}\right.
$$

where $\Omega \epsilon\{X\}, X>0, t$ is the tissue overall thickness, $t_{\text {skin }}$ is the skin layer thickness.

Accuracy in the estimated parameters depends on the following measures. Firstly, the thermal problem of a particular organ to be addressed will be selected because it is obvious that, for a particular organ, a tumor could grow in a certain area. For example, in the chest assuming the muscle tissue layer is prone to grow a tumor, therefore, some constraints can be imposed to put the solution confined into that region and hence the generalized solution domain can be expressed as,

$$
\Gamma \subset\left\{h, R, T_{c}\right\}
$$

and the constraints for the chest tumor parameters are,

$$
\Gamma=\left\{\begin{array}{ccc}
h & 1 \leq h \leq 4 & \mathrm{~cm} \\
R & 3 \leq R \leq 15 & \mathrm{~mm} \\
T_{c} & 309.15^{\circ} \leq T_{c} \leq 313.15 & \mathrm{~K}
\end{array}\right.
$$

Secondly, a dimensionless solution vector is created because parameters such as tumor depth, radius and tumor center temperature depend on the body geometry and they hold diverse ranges, for example, in particular case of the chest tumor, the depth may lie between 1 and 4 centimetres, the center temperature between 0.2 and 4 degrees higher than the healthy tissues and the radius between three and fifteen millimetres. Therefore, a simple and efficient way to fit the assorted elements for GPM is to define a dimensionless solution vector as:

$$
\Gamma_{n}=\left\{\begin{array}{l}
h_{n} \\
R_{n} \\
T_{c_{n}}
\end{array}\right.
$$


where $\Gamma_{n} \in \mathbb{R}, 1 \leq \mathbb{R} \leq 2$, is the dimensionless domain and $h_{n}, R_{n}$ and $T_{c_{n}}$ are the dimensionless tumor depth, radius and center temperature, respectively.

Thirdly, after optimizing the thermal problem the dimensionless solution vector will be converted to the real parameter value. The reproduction of actual heat source parameter from the dimensionless parameter the following formula will be used:

$$
x=\left(x_{\max }-x_{\min }\right)\left(x_{n}-1\right)+x_{\min }
$$

where $\left\{\mathcal{X}, x_{\max }, \mathcal{X}_{\min }\right\} \in \Gamma, \Gamma \subset\left\{h, T_{c}, R\right\}$ and $\left\{\mathcal{X}_{n}\right\} \in \Gamma_{n}$.

Finally, the overall process has been established with MATLAB where the dimentionalization, dynamic gradient computations, search direction defeminisation, actual parameter computation and best solution selection are performed by the script with the reparative solution of heat-source models and converging to the test pattern.

The optimization approaches to determine the thermal and geometrical parameters of tumors from their local surface temperature profiles are discussed as follows:

\subsubsection{Gradient Computations}

To find the search direction in GPM, the computation of gradient of the function (rate of change in temperature with model variable) is very important. The gradient vector $\nabla T\left(h, T_{c}, R\right)=\left[\begin{array}{c}\frac{\partial T}{\partial h} \\ \frac{\partial T}{\partial T_{c}} \\ \frac{\partial T}{\partial R}\end{array}\right]$ can be computed mathematically or graphically. Before applying the optimization process, the change in temperature profiles with model parameter has been investigated graphically. The temperature distribution along a line passing the point which is directly over the heat source centre is computed while with different values of depth, radius, and heat-rate ratio.

The temperature plots for heat sources of various radius are shown in Figure 6-7 where the top window shows the temperature graphs for several radius between $0.9 \mathrm{~mm}$ and $20 \mathrm{~mm}$ and the bottom window indicates the rate of change in temperature with radius at three significant points at the coldest point (name it $d R / d T_{\min }$ ), at the hottest point (name it $d R / d T_{\text {mid }}$ ), and in-between 
(name it $d R / d T_{\max }$ ), them. The figure demonstrates that the slope of the curve is not identical everywhere but rather increases if the surface temperature goes higher.

Temperature plots if the heat source depth changes are shown in Figure 6-8 where it is seen from the up window that the deep-seated source decreases the maximum elevation but increases the base area over which the temperature hill grows which is clearly depicted in the bottom window where the gradient is positive at apex, and negative at the colder area.
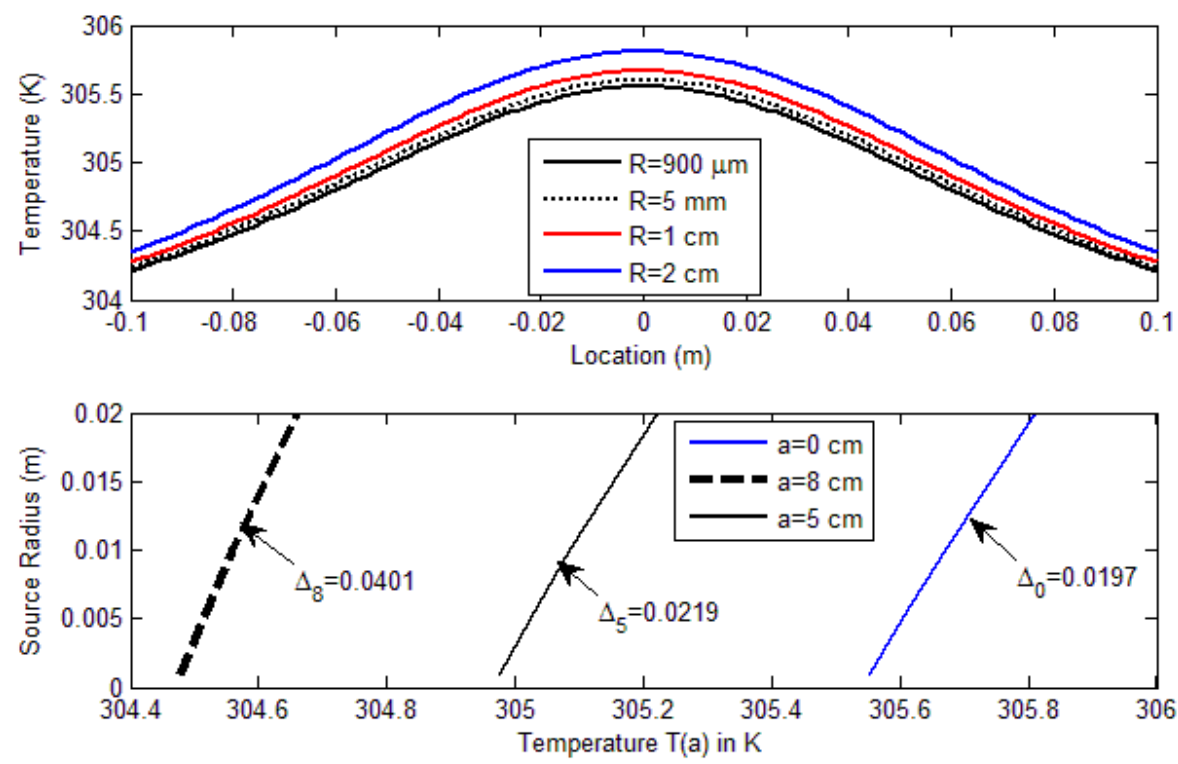

Figure 6-7Heat source model responses to $R$ and respective slopes of $\frac{d T(a)}{d R}$

The temperature graphs for different tumor-core temperature are presented in Figure 6-9 where the top window presents the temperature profiles for different body temperature between $309.15 \mathrm{~K}$ and $313.15 \mathrm{~K}$ and the bottom window shows the slope of the temperature change rates at the maximum temperature points. The figure shows that the slope is uniform for all values of heatrate ratios.

In Figure 6-7, it can be seen that increased heat source radius elevates the maximum temperature magnitude and the region over which local temperature grows. The analysis shows that a reduction of $55 \%$ of a $4 \mathrm{~mm}$ diameter heat source, lowers almost $1 \mathrm{~K}$ in maximum temperature and decreases $26 \%$ of subtended area over which local temperature (assuming the region having at least $303 \mathrm{~K}$ as a local hot zone) elevation exists on skin for a source in $3.5 \mathrm{~cm}$ depth and of $310.15 \mathrm{~K}$ of body core temperature. The bottom window reveals the slopes at different 
points on $R v s . T$ curves that are required for radius estimation from surface temperature pattern as proposed in Eq. 6.3.
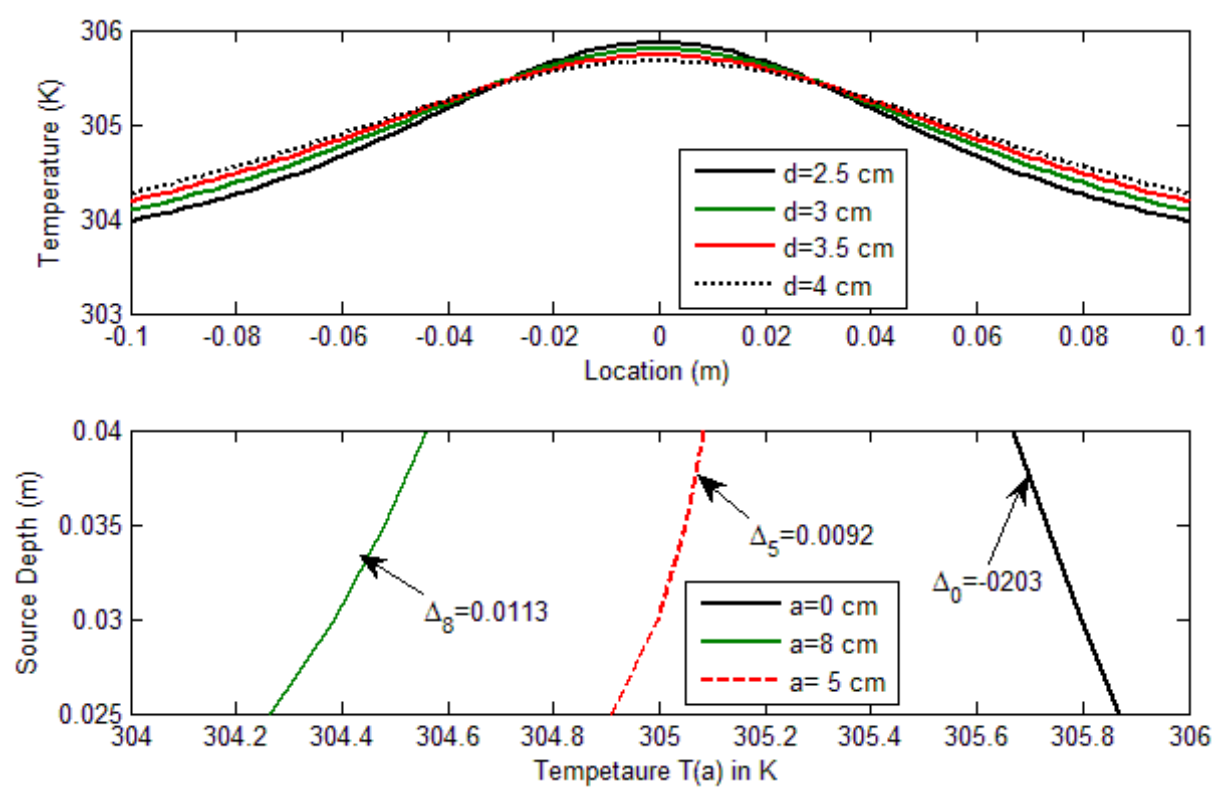

Figure 6-8 Heat source model responses to $h$ and slopes of $\frac{d T(a)}{d h}$

From Figure 6-9, it can be observed that $1 \mathrm{~K}$ change in the heat source temperature notices for approximately $0.8 \mathrm{~K}$ change in maximum skin temperature for a source of $5 \mathrm{~mm}$ radius in 3.5 $\mathrm{cm}$ depth. It is evident that benign to malignant is easily separable. The slope of the $T_{c} v s . T_{\max }$ curve is also presented to use for parameter estimator algorithm.
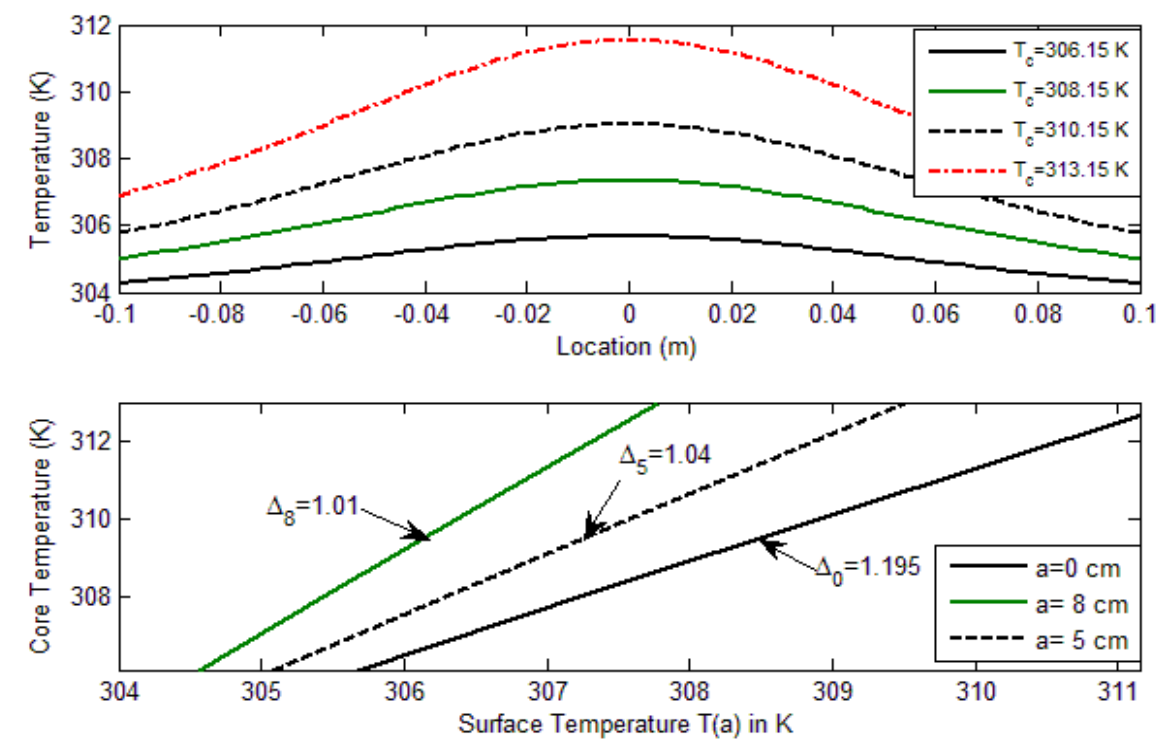

Figure 6-9Heat source model responses to $T_{C}$ and slopes of $\frac{d T(a)}{d T_{c}}$ 
From the above analysis, it is seen that $d$ playes a substantial role on $T( \pm 0.1)$ while $T_{c}$ plays on $T(0)$ but $R$ has weak effect on $T$ in comparing with $d$ and $T_{c}$. The proposed linear mapping convergent algorithm basically based on the slopes of parameter's effect on surface temperature curves are used to estimate the tumor parameters. Typical sample data set could be obtained from thermogram, numerical result or heat source model. Three sample points on the surface temperature plot have been chosen to estimate the average slope to develop the pattern estimator algorithm.

The above analysis shows that temperature gradient due to the heat source parameter is not static and changes with space. Therefore, the proposed GPM approach computes slopes in different points and changes the search parameter as follows:

The search parameter updating algorithm is developed based on equations and constraints presented in Eqn. 6.18-6.20 (for parameter renewal) and in Eqn. 6.17 (for limiting). A convergent algorithm has been developed employing error minimization technique for parameter estimation.

$$
\begin{aligned}
& h_{i}=h_{i-1}+h_{i-1}\left(\frac{\Delta h}{\nabla T_{\max }}\right)_{i d e a l}\left(T_{\min \_s, i-1_{1}}-T_{\min _{-} a}\right) \\
& T_{c, i}=T_{c, i-1}+\left(\frac{\Delta T_{C}}{\nabla T_{\min }}\right)_{i d e a l}\left(T_{\max _{-}, i-1}-T_{\text {max }_{-} a}\right) \\
& R_{i}=R_{i-1}+R_{i-1}\left[\left(\frac{\Delta R}{\nabla T_{\max }}\right)_{i d e a l}\left(T_{\text {max } \_s, i-1-T_{\text {max } \_} a}\right)+\right. \\
& \left.\left(\frac{\Delta R}{\nabla T_{\text {min }}}\right)_{\text {ideal }}\left(T_{\text {min_s }, i-1_{1}}-T_{\text {min_a }_{-} a}\right)+\left(\frac{\Delta R}{\nabla T_{\text {mid }}}\right)_{\text {ideal }}\left(T_{\text {mid_s, } i-1}-T_{\text {mid_a }}\right)\right]
\end{aligned}
$$

where, $i=1,2 \ldots \ldots, N_{.}$, and $T_{\text {max }_{-}, i}, T_{\text {min_s, } i}$, and $T_{\text {mid_s }, i}$ are the maximum, minimum and midpoint temperature of simulated pattern at $\mathrm{i}^{\text {th }}$ iteration and $T_{\text {max } a} a, T_{\text {min_a }} a$ and $T_{\text {mid_a }}$ are the maximum, minimum and mid-point temperature on actual pattern, respectively; $\left(\frac{\nabla X}{\nabla T_{S}}\right)_{\text {ideal }}$ is the slope of the $x$ vs. $T_{S}$ where $x$ is $d$ or $R$ or $T_{c}$ and $T_{S}$ is the maximum or minimum or midpoint temperature. The slopes are determined incorporating Fig. 6.5, 6.6 and 6.7. In this proposed method the $h$ and $T_{c}$ is determined by minimizing the minimum and maximum point error, respectively (serve the very similar purpose of the 'COARSE' knob of an instrument) whereas the $R$ assumed as 'FINE' knob to adjust (tune).

To limit the solution domain, assume 


$$
\begin{gathered}
d_{\text {skin }} \leq h \leq d_{d v r} \\
T_{a} \leq T_{c} \leq T_{\text {cmax }} \text { alternatively } Q_{m, \text { healthy }} \leq Q_{m} \leq n Q_{m, \text { healthy }} \\
R_{\text {cell }} \leq R \leq d_{d v r}-t_{\text {skin }}
\end{gathered}
$$

where $d_{\text {skin }}=0.91 \pm 0.14 \mathrm{~mm}$, is the usual thickness of forearm skin and $d_{d v r}=4 \mathrm{~cm}$, is the depth of the deep volar at 'radius' side, within this range the tumor could grow, $Q_{m}$ defines the range of metabolic heat generation in healthy to cancerous tissues $(n=40), T_{a}$ is the artier blood temperature $(=310.15 \mathrm{~K}), \quad T_{\text {cmax }}$ could be deduced from upper limit of $Q_{m}$ and $R$ is the radius bounded by unit cell $(200 \mu m)$ to the depth constraints.

\subsubsection{Optimization Process with GPM}

This section exemplifies the process of optimizing the thermal problem due to a chest tumor. Using the concept discussed in Section 0, a heat source model of a chest tumor is produced where the tissue depth is assumed $3.8 \mathrm{~cm}$. The governing equation of the model is a function of tumor depth $(h)$, radius $(R)$ and metabolic rate ratio $(n)$ which can be seen in Eqn. 6.23 after rewriting Eqn. 6.3 with help of Eqn. 6.4 to 6.6,

$$
T(h, R, n, a)=T_{e}+\frac{\left(-0.111 h^{2}+0.64 h+34\right) e^{10^{-4}(6 n+33.4 R)}-t_{e}}{\frac{2000 h a}{k R}(R n)^{-0.92}\left\{(h-2.2)^{2}+2.6\right\}\left(h^{2}+a^{2}-R \sqrt{h^{2}+a^{2}}\right)+1}
$$

The expressions for gradients of Eqn. 6.23 in terms of depth, radius and heat-rate ratio are presented below.

$$
\begin{gathered}
\frac{\partial T}{\partial h}=\frac{V e^{10^{-4}(6 n+33.4 R)}(-0.222 h+0.64)-U\left[\frac{20 h_{a}}{k R}(R n)^{-0.92}\left((2 h-4.4)\left(2 h-\frac{R h}{\sqrt{h^{2}+a^{2}}}\right)\right)\right]}{V^{2}} \\
\frac{\partial T}{\partial R}=\frac{V\left(33.4 \times 10^{-4}\right) e^{10^{-4}(6 n+33.4 R)}\left(-0.111 h^{2}+0.64 h+34\right)-U\left[\frac{2000 h_{a}}{k R^{2}}(R n)^{-0.92}\left\{(h-2.2)^{2}+2.6\right\}\left(\frac{0.92 \sqrt{h^{2}+a^{2}}}{R}-1.92\right)\right]}{V^{2}} \\
\frac{\partial T}{\partial n}=\frac{V\left(6 \times 10^{-4}\right) e^{10^{-4}(6 n+33.4 R)}\left(-0.111 h^{2}+0.64 h+34\right)-U\left[\frac{1840 h_{a}}{k R n}(R n)^{-0.92}\left\{(h-2.2)^{2}+2.6\right\}\left(1-R \sqrt{h^{2}+a^{2}}\right)\right]}{V^{2}}
\end{gathered}
$$

where $V=\frac{2000 h_{a}}{k R}(R n)^{-0.92}\left\{(h-2.2)^{2}+2.6\right\}\left(1-R \sqrt{h^{2}+a^{2}}\right)+1$ and

$$
U=\left(-0.111 h^{2}+0.64 h+34\right) e^{10^{-4}(6 n+33.4 R)}-t_{e}
$$


Starting with a maximum cost function, the heat source is assumed placed at the deepest tissue, the size and heat-rate ratio of heat source has the minimum value. For this case, the boundary conditions are,

$$
\left\{\begin{array}{c}
g_{1}=h-R \geq 0 \\
g_{2}=R \geq 6 \\
g_{3}=n-120 \leq 0
\end{array}\right.
$$

Therefore,

$$
\begin{aligned}
& \frac{\partial g_{1}}{\partial h}=1, \frac{\partial g_{1}}{\partial R}=-1, \frac{\partial g_{1}}{\partial n}=0 \\
& \frac{\partial g_{2}}{\partial h}=0, \frac{\partial g_{2}}{\partial R}=1, \frac{\partial g_{1}}{\partial n}=0 \\
& \frac{\partial g_{3}}{\partial h}=0, \frac{\partial g_{3}}{\partial R}=0, \frac{\partial g_{3}}{\partial n}=1
\end{aligned}
$$

Then

$$
\begin{gathered}
N=\left[\begin{array}{ccc}
1 & -1 & 0 \\
0 & 1 & 0 \\
0 & 0 & 1
\end{array}\right], N^{T} N=\left[\begin{array}{ccc}
1 & -1 & 0 \\
-1 & 2 & 0 \\
0 & 0 & 1
\end{array}\right],\left(N^{T} N\right)^{-1}=\left[\begin{array}{lll}
2 & 1 & 0 \\
1 & 1 & 0 \\
0 & 0 & 1
\end{array}\right], \\
\lambda=N\left(N^{T} N\right)^{-1} N^{T}=\left[\begin{array}{lll}
1 & 0 & 0 \\
0 & 1 & 0 \\
0 & 0 & 1
\end{array}\right] \\
P=I-N\left(N^{T} N\right)^{-1} N^{T}=\left[\begin{array}{lll}
0 & 0 & 0 \\
0 & 0 & 0 \\
0 & 0 & 0
\end{array}\right]
\end{gathered}
$$

Where $P \nabla T=0$ satisfies the Kuhn-Tucker conditions, therefore, there is an exact solution for the problem.

Assuming the start point at $X_{0}^{T}=(3.2,6,5)$, the

$$
\nabla T=\left\{\begin{array}{c}
0.0113 \\
0.0401 \\
1.195
\end{array}\right\}_{\text {min }},\left\{\begin{array}{c}
0.0203 \\
0.0219 \\
1.195
\end{array}\right\}_{\text {mid }},\left\{\begin{array}{c}
0.0203 \\
0.0197 \\
1.195
\end{array}\right\}_{\text {max }}
$$

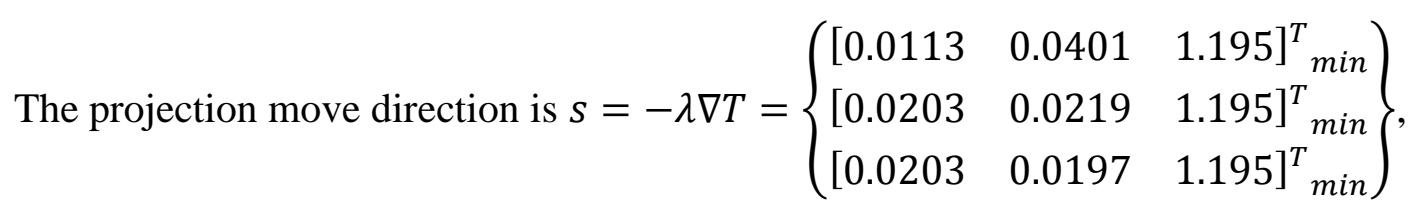


For $10 \%$ improvement in the objective function $\gamma=0.1$ and the exploratory move is

$$
\alpha=-\frac{0.1 T}{S^{T} \nabla T}=-\frac{0.1 \times 5}{-8}=0.0625
$$

Without considering the correction move, the projection and restoration moves as,

$$
X_{1}=\left\{\begin{array}{c}
3.2 \\
6 \\
5
\end{array}\right\}+0.0625\left\{\begin{array}{c}
-0.0133 \\
0.02 \\
1.195
\end{array}\right\}=\left\{\begin{array}{c}
-0.0133 \\
0.02 \\
1.195
\end{array}\right\}
$$

The initial value of the heat source depth $d_{0}$ can be calculated from Eq. (6.13) though it varies significantly with the location of arbitrary point $a$. A distant point closer to the demarcation line between the hot zone and sound body has been chosen to get optimum $d_{0}$. The initial value of $T_{c 0}$ is computed using Eq. (6.9) with assuming $R_{0}=0.0002 \mathrm{~m}$. A convergent algorithm has been developed employing error minimization technique for parameter estimation.

To determine the exact values to the parameters the study proposes the following algorithm:

1. Initialize the parameters (use $\mathrm{Eq}$ (6.7) and (6.13) with lower limit of radius, say $R=$ $0.0002 \mathrm{~m}$ )

2. Calculate $T_{\text {max,model }}$ using model governing equation

3. Upgrade $d$ using Eq. 6.14

a. with holding within the range (i.e. if $h<h_{\min }$ then $h=h_{\min }$ or if $d>$ $h_{\max }$ then $h=h_{\max }$ ), or

b. with keeping the previous smaller value (i.e. if $h_{i}>h_{i-1}$ then $h_{i}=h_{i-1}$ )

c. otherwise continue the up gradation for $\varepsilon>$ error or iteration $\#<N$

4. Recalculate $T_{c}$ using Eq. 6.15 with current value of $d$

a. with keeping within the range (i.e. if $T_{c}>T_{c, \max }$ then $T_{c}=T_{c, \max }$ or if $T_{c}<$ $T_{\text {sample,max }}$ then $\left.T_{c}=T_{\text {sample,max }}\right)$

b. otherwise continue this Step-4 for $\varepsilon>$ error or iteration $\#<N$

5. Renew $R$ using Eq. 6.16 with current value of $d$ and $T_{c}$

a. with abiding by the range (i.e. if $R>h-h_{\min }$ then $R=h-h_{\min }$ or if $R<$ $R_{\text {cell }}$ then $R=R_{\text {cell }}$ )

b. otherwise continue the Step-5 for $\varepsilon>$ error or iteration $\#<N$

6. If two parameters become constant then go for next solution (decrease $d$ by 5\%) 
7. Repeat Steps 3 to 5 until $h, T_{c}$ and $R$ become constant

8. Stop

These procedures are programed in MATLAB to predict the heat source parameters $\left(T_{c}, h\right.$ and $\left.R\right)$ from surface temperature image.

\subsubsection{Result}

The estimation process is applied for computing significant parameters of hot nodule. As discussed above, the approach requires a governing equation, bound constraints set, and a target temperature pattern. Of them, the first two are discussed in the previous sections, which are programed with MATLAB according to the GPM algorithm. The target temperature matrix should be captured on a patient's chest, which is complex and time consuming and needs to be developed in a controlled laboratory more likely than in a hospital, where thermogram will take place. In lieu of real thermogram, the study has used numerical simulated temperature data over the anatomic accurate chest model where the numerical data has also been imposed with some random noise to produce a kind of realistic target matrix.

The obtained result is presented in Table 6-1. The process has been repeated for thee 'Samples' - the 'Sample-I' small-size, moderate heat-rate, mid-way tumor has radius 6mm, volumetric metabolic rate $10 \mathrm{~kW}$ and grown in $3 \mathrm{~cm}$ deep. The optimization approach estimates depth and radius exactly but only $0.07 \%$ overestimates in metabolic heat generation. Diagnosis of a deep-seated benign tumor (Sample-II) shows the error-free depth estimation while $2.03 \%$ error in heat generation rate and $1.11 \%$ in size measurement. Similar to the first case, the shallow-body tumor of moderate heat generation rate and $1 \mathrm{~cm}$ radius (Sample-III) provides true results for depth and radius measurement while $1.44 \%$ error in predicting the heat generation rate. 
Table 6-1 Estimated heat source parameters

\begin{tabular}{|c|c|c|c|c|}
\hline & Parameter & Target Value & Estimated Value & Avg. Error \\
\hline Sample -I & $h$ & $0.03 \mathrm{~m}$ & $0.030 \mathrm{~m}$ & 0 \\
\cline { 2 - 5 } & $Q_{m}$ & $10208 \mathrm{~W} / \mathrm{m}^{3}$ & $10215 \mathrm{~W} / \mathrm{m}^{3}$ & 0.07 \\
\cline { 2 - 5 } & $R$ & $0.006 \mathrm{~m}$ & $0.006 \mathrm{~m}$ & 0 \\
\hline \multirow{3}{*}{ Sample-II } & $h$ & $0.038 \mathrm{~m}$ & $0.038 \mathrm{~m}$ & 0 \\
\cline { 2 - 5 } & $Q_{m}$ & $3783 \mathrm{~W} / \mathrm{m}^{3}$ & $3860 \mathrm{~W} / \mathrm{m}^{3}$ & 2.03 \\
\cline { 2 - 5 } & $R$ & $0.009 \mathrm{~m}$ & $0.0089 \mathrm{~m}$ & 1.11 \\
\hline Sample-III & $h$ & $0.025 \mathrm{~m}$ & $0.025 \mathrm{~m}$ & 0 \\
\cline { 2 - 5 } & $Q_{m}$ & $9816 \mathrm{~W} / \mathrm{m}^{3}$ & $9675 \mathrm{~W} / \mathrm{m}^{3}$ & 1.44 \\
\cline { 2 - 5 } & $R$ & $0.01 \mathrm{~m}$ & $0.01 \mathrm{~m}$ & 0 \\
\hline
\end{tabular}

The GPM requires partial derivatives of the surface temperature plot to the model parameters which requires a continuous differentiable governing equation that has been met with the proposed heat source model. However, the heat-source model has derived from the numerical results obtained on simplistic (homogenous) tissue models. The optimization problems on realistic models require solving the bio-thermal problem numerically which is a desecrate process and unable to compute the gradients.

\subsubsection{Pattern Search Method (PSM)}

Unlike the gradient projection method (GPM), the pattern search method (PSM) works without accomplishing the gradient vector and the directional derivatives and can be applied on discontinuous or non-differentiable functions. Such optimization methods are also known as direct-search, derivative-free, or black-box methods. The technical components of the general PSM for the linearly constraints problem are detailed in [66, 65]. Description of the useful components and their nature for solving the mentioned optimization problem are following. 
The pattern $\left(P_{k}\right)$ is consisting of a basis matrix $\left(B \in R^{n \times n}\right)$ and a generating matrix $\left(C_{k}=\left[\begin{array}{ll}\Gamma_{k} & L_{k}\end{array}\right]\right)$. In the two column generating matrix, the column $\Gamma_{k}$ contains a finite set of nonsingular matrices with both positive and negative signs and the column matrix $L_{k}$ contains a column of zeros.

Trial step $s_{k}^{i}$ vector is defined as $s_{k}^{i}=\Delta_{k} p_{k}^{i}$ which is the trial vector at $k$-th iteration for some $i \in\{1,2, \ldots . n\}$, with $\Delta_{k} \in \mathbb{R}, \Delta_{k}>0$ and the $p_{k}^{i}$ denotes the $i$-th column of $P_{k}=B c_{k}^{i}$ where $c_{k}^{i}$ denotes a column of $C_{k}=\left[\begin{array}{llll}c_{k}^{1} & \ldots & c_{k}^{p}\end{array}\right]$. A feasible trial step is determined by $\left(x_{k}+s_{k}^{i}\right) \in \Omega$, where $x_{k} \in X \subset R^{n}$ is the current iterate. Any point of the form $x_{k}^{i}=x_{k}+s_{k}^{i}$ is called a trial point.

PSM proceed by conducting a series of exploratory moves about the current iterate $x_{k}$ to choose a new iterate $x_{k+1}=x_{k}+s_{k}$, for some feasible step $s_{k}$ determined during the course of exploratory moves. The 'moves' required for converging to a local or to the global minima is obtained with fulfilling the following conditions:

1. $s_{k} \in \Delta_{k} P_{k}$

2. $\left(x_{k}+s_{k}\right) \in \Omega$

3. If $\min \left\{f\left(x_{k}+y\right) \mid y \in \Delta_{k} P_{k}, x_{k}+y \in \Omega\right\}<f\left(x_{k}\right)$, then $f\left(x_{k}+s_{k}\right)<f\left(x_{k}\right)$

By abuse of notation, if $A$ is a matrix, $y \in A$ means that the vector $y$ is a column of $A$.

The PSM requires defining a column matrix of initial value and positive step-size parameter as:

Let $X_{0}=\left|\begin{array}{c}x_{1} \\ x_{2} \\ \vdots \\ x_{n}\end{array}\right| \in \Omega$ and $\Delta_{0}>0$ and a direction matrix $P_{k}$

Then the process goes in the following way:

For $k=0,1,2, \ldots \ldots$

1. Compute $f\left(x_{k}\right)$.

2. Determine $s_{k}$ abiding by $s_{k} \in \Delta_{k} P_{k},\left(x_{k}+s_{k}\right) \in \Omega$ and if $\min \left\{f\left(x_{k}+y\right) \mid y \in\right.$ $\left.\Delta_{k} P_{k}, x_{k}+y \in \Omega\right\}<f\left(x_{k}\right)$, then $f\left(x_{k}+s_{k}\right)<f\left(x_{k}\right)$.

3. If $f\left(x_{k}+s_{k}\right)<f\left(x_{k}\right)$, then $x_{k+1}=x_{k}+s_{k}$. Otherwise $x_{k+1}=x_{k}$. 
4. If $f\left(x_{k}+s_{k}\right) \geq f\left(x_{k}\right)$ [i.e. an unsuccessful search], then $\Delta_{k+1}=\theta_{\mathrm{k}} \Delta_{\mathrm{k}}$, where $\theta_{\mathrm{k}} \in$ $(0,1)$. Otherwise (for a successful search) $\Delta_{k+1}=\lambda_{\mathrm{k}} \Delta_{\mathrm{k}}$ where $\lambda_{\mathrm{k}} \in(1,+\infty]$.

Therefore in PSM, the basis matrix $B$, the generating matrix $C_{k}$, the exploratory moves to produce a feasible step $s_{k}$ and the updating of $C_{k}$ and $\Delta_{k}$ must be specified.

\subsubsection{PSM Process}

The PSM will compute the basis matrix, direction matrix, exploratory moves and updating parameters and step sizes. It is discovered that tumors could affect at least four behaviors of the tissues, those that are associated with heat generation and dissipation. Therefore, the spatial distribution of temperature on the skin surface is an obvious function of blood perfusion, metabolism, tumor location and tumor size, which can be written as:

$$
T(X)=f\left(\omega_{b}, h, Q_{m}, R\right)
$$

With the pattern search algorithm for bound constraint optimization minimize the fitness functions defined before which can be rewritten for each function as:

$$
\min || T_{o}-T_{m}\left(\omega_{b}, h, Q_{m}, R\right) \|
$$

Subject to $x \subset\left\{h, Q_{m}, R\right\} \in \Omega$, where $\Omega$ is the region over which the solution is bounded. $T_{o}$ is the actual temperature pattern over the skin surface that may be obtained from thermogram (using infrared camera, numerical simulation etc) and $T_{m}(X)$ is the estimated temperature pattern that is obtained from the model expression (or directly from the numerical models).

The solution matrix may not be unique because several solutions could provide similar results. However, application of PSM for thermal problem optimization in tumor requires some special techniques in handling parameters which include understanding the solution domain, normalizing and demoralizing the parameters, properly choosing the initial value matrix. 
Table 6-2 Initial value matrix

\begin{tabular}{|c|c|c|c|c|}
\hline & Set-1 & Set-2 & Set-3 & Set-4 \\
\hline$\omega_{b 0}$ & 1.2 & 1.2 & 1.5 & 1.2 \\
\hline$h_{0}$ & 1.2 & 1.2 & 1.8 & 1.8 \\
\hline$Q_{m 0}$ & 1.2 & 1.5 & 1.5 & 1.2 \\
\hline$R_{0}$ & 1.2 & 1.3 & 1.5 & 1.2 \\
\hline & $\begin{array}{c}\text { Midway- } \\
\text { mid stage }\end{array}$ & $\begin{array}{c}\text { Shallow- } \\
\text { malignant }\end{array}$ & $\begin{array}{c}\text { Deep seated- } \\
\text { benign }\end{array}$ & $\begin{array}{c}\text { Shallow- } \\
\text { benign }\end{array}$ \\
\hline
\end{tabular}

The solution domain, $\Omega=\left\{\begin{array}{c}\omega_{b} \quad 0.5 \leq \omega_{b} \leq 160 \\ h \quad 0.35-R \leq h \leq 6-R \\ Q_{m} \quad 5 \leq Q_{m} \leq 100 k \\ R \quad 0.6 \leq R \leq h / 2\end{array}\right.$ where the parameters in $\Omega$ have diverse ranges, for example, the depth of shallow tumors could be a fraction centimetre whereas of deep-seated tumors the depth could be few centimetres, likewise the tumor center temperature could vary between five and hundred kilowatt per unit $\mathrm{m}^{3}$, the radius between millimetre and centimetre and the perfusion rate varies as low as 0.5 to maximum 160. A simple and efficient way to fit the assorted elements for optimization is to define a dimensionless vector as:

$$
\Omega=\left\{\begin{array}{c}
\omega_{b n} \\
h_{n} \\
Q_{m n} \\
R_{n}
\end{array} \quad \text { where } \Omega_{n} \in \mathbb{R}, 1 \leq \mathbb{R} \leq 2, \text { is the dimensionless domain and } X_{n} \subset\right.
$$

$\left\{\omega_{b n}, h_{n}, Q_{m n}, R_{n}\right\}$ are the dimensionless parameters. In further analysis the range of the variables are kept between 1 and 2 and the generalized domain expression could be rewritten as, $\Omega=1 \leq$ $X \subset\left\{h_{n}, T_{c n}, R_{n}\right\} \leq 2$. The actual parameter values in a specific organ could be reproduced from the dimensionless (normalized) parameter using the following formula:

$$
x=\left(x_{\max }-x_{\min }\right)\left(x_{n}-1\right)+x_{\min }
$$

where $\left\{\mathcal{X}, x_{\max }, \mathcal{X}_{\min }\right\} \in \Omega, \Omega \in \mathrm{X} \subset\left\{\omega_{b}, d, Q_{m}, R\right\}$ and $\left\{\mathcal{X}_{n}\right\} \in \Omega_{n}$.

Selection of initialization matrix (i.e. $X_{0}$ and $\Delta_{0}$ ) can lead to local or global minima after reasonable iterations. After a series of successful computation, several choices $x_{0}$ of and their respective $\Delta_{0}$ was recommended to ensure the global minima for specific types of tumors. The deep-seated to shallow body and benign to malignant tumors have some distinguishable features 
which might be reflected in their temperature pattern. The research considers those features while choosing set of initial values. Examples of initial value sets are listed in Table 6-2.

The optimization approach calculates the followings:

The pattern $\left(P_{k}=B\left[\begin{array}{ll}\Gamma_{k} & L_{k}\end{array}\right]\right)$, where

The generating matrix,

$$
\pm \Gamma_{k}= \pm\left|\begin{array}{lllllllllllllll}
1 & 0 & 1 & 0 & 1 & 0 & 1 & 0 & 1 & 0 & 1 & 0 & 1 & 0 & 1 \\
0 & 1 & 1 & 0 & 0 & 1 & 1 & 0 & 0 & 1 & 1 & 0 & 0 & 1 & 1 \\
0 & 0 & 0 & 1 & 1 & 1 & 1 & 0 & 0 & 0 & 0 & 1 & 1 & 1 & 1 \\
0 & 0 & 0 & 0 & 0 & 0 & 0 & 1 & 1 & 1 & 1 & 1 & 1 & 1 & 1
\end{array}\right|
$$

The column matrix, $L_{k}=\left|\begin{array}{l}0 \\ 0 \\ 0 \\ 0\end{array}\right|$

The Basis vector, $B=\left|\begin{array}{llll}1 & 1 & 1 & 1\end{array}\right|$

The pattern matrix leads the search into 15 different directions, where each column in the matrix indicates the particular direction. The search algorithm featured is to determine the appropriate search directions by reordering the columns. In case of no forward or backward move, the algorithm will update the trail steps for further moves.

Assuming a breast tumor, the initial parameter matrix $X^{T}=\left|\begin{array}{llll}0.52 & 0.06 & 5000 & 0.006\end{array}\right| \in$ $\Omega$ and the trail step size $\Delta_{0}>0.05$.

Trial step vector $\left(s_{k}^{i}=\Delta_{k} P_{k}^{i}\right)$ at $k$-th iteration for some $i \in\{1,2, \ldots .15\}, \Delta_{k} \in \mathbb{R}$ with $\Delta_{k}>0$ and $P_{k}^{i}$ denotes the $i-$ th column of $P_{k}$. For example for the first iteration $(k=1)$ to the $5^{\text {th }}$ direction $(i=5)$, the step vector $s_{1}^{5}=\left|\begin{array}{llll}0.05 & 0.05 & 0.05 & 0\end{array}\right|^{T}$ and the trial step is determined by $|0.57 \quad 0.065 \quad 5000.05 \quad 0.0065| \in \Omega$, where $X_{k} \in x \subset\left\{\omega_{b}, d, Q_{m}, R\right\}$ is the current iterate.

The exploratory move required converging to a local or to the global minima is obtained with fulfilling the following conditions:

$$
|| T_{o}-T_{m}^{k}\left(x_{k}\right)||>|| T_{o}-T_{m}^{k+1}\left(x_{k+1}\right)|| \text { with } s_{k} \in \Delta_{k} P_{k} \text { and }\left(x_{k}+s_{k}\right) \in \Omega \text { where } x_{k+1}=
$$
$x_{k}+s_{k}$.

The search method for the particular problem goes in the following way: 
For $k=0,1,2, \ldots \ldots$.

For $i=1,2, \ldots \ldots 15$,

a) Compute ||$T_{o}-T_{m}^{k}\left(x_{k}^{i}\right)||$.

b) Determine $s_{k}^{i} \quad$ abiding by $s_{k}^{i} \in \Delta_{k} P_{k}^{i},\left(x_{k}+s_{k}^{i}\right) \in \Omega$ and ||$T_{o}-T_{s}^{k}\left(x_{k}\right)||>$ ||$T_{a}-T_{s}^{k+1}\left(x_{k+1}\right)||$ and perform step (a).

c) For all values of $i$ compute $\min || T_{a}-T_{s}^{k}\left(x_{k+1}\right)||$ and find $x_{k}=x_{k+1}$ and $\Delta_{k+1}=\Delta_{k} / 2$ (keep not less than 0.005$)$. Otherwise $x$ remain unchanged and $\Delta_{k+1}=2 \Delta_{k}$

\subsubsection{Result}

The perfusion rate, depth, metabolic rate and radius of unknown tumors have been estimated with the PSM. Tumors with different physio-thermo-biological parameters grown in the human chest, forearm and breast have been investigated. The entire estimation approach has combined two processes - the numerical process to solve the bio-heat equation on the anatomic accurate model and the optimization process to evolve solution vectors and to find the best solution(s). For chest tumors the numerical simulation is performed with FEM, where the programs are written in MATLAB. However, for forearm and breast tumors the bio-heat problems are solved numerically with finite element (FE) software, which has required a link between the numerical simulation data and the PSM script. Consequently, this has increased the run time and the computation memory. The target matrix is generated from FE data which has been obtained by solving the Penne's heat equation on the realistic model. Such generated ideal target matrix is called the 'Noiseless System' while the 'Noisy System' is that which has added random noises.

The estimated perfusion rates, depths, heat-rates, and radii for the noiseless system for chest, forearm and breast tumors are presented in Table 6-3. The surface thermal behaviour of the chest tumor of $18 \mathrm{~mm}$ diameter and volumetric heat generation rate of $40 \mathrm{~kW}$ (is grown in $2.5 \mathrm{~cm}$ under the skin surface has been estimated numerically to develop the target matrix. Then the PSM is executed for the all four (see Table 6-2) initial test vector sets. For 'set-I' the estimate is found $2.4977 \mathrm{~cm}, 40.221 \mathrm{~kW} / \mathrm{m}^{3}$ and $9.0072 \mathrm{~mm}$ for depth, metabolic rate and radius, respectively. The estimated values for other sets of initial values are also presented in the table. The chest tumor 
diagnosis system gets the highest error while computing the radius for initial value 'Set-3'. Another target matrix of surface temperature is considered for a $24 \mathrm{~mm}$-diameter, $50 \mathrm{~kW} / \mathrm{m}^{3}$ heat rate and $1.6 \mathrm{~cm}$ deep forearm tumor. The parameters of such tumor are calculated for the initial value sets and the estimated results are presented in the table. This time the maximum error is received while calculating the depth with set 1 . The optimization process is also applied to a diagnosis a tiny (radius $8 \mathrm{~mm}$ ), shallow body (depth $12 \mathrm{~mm}$ ) breast malignant (volumetric heat rate $65 \mathrm{~kW})$. Detection of such malignancy with the above mentioned initial value sets contains $0.61 \%$ error while predicting the metabolic heat rate with set-3.

In case of real thermogram, the captured thermal image is not as ideal as the numerical data computed in the noiseless system. Therefore, the target matrix can be incredibly different from that assumed above. Mimicking the realistic thermal surface data in developing the target matrix is achieved by adding random noise. The imposed noise is kept between 0 and 10\%. The parameters are estimated for the very same tumors in chest, forearm and breast and the results are presented in Table 6-4. It is seen that the estimation error is slightly higher in noisy system than the noise-less system. The source of un-symmetric temperature pattern in real thermogram can be generated from the irregular shape and margin of tumor.

The computational complexity of this algorithm is on the order of $O\left(n^{2}\right)$, this is simply because of the algorithm has two "for" loops that are repeated for " $n$ " times each. 
Table 6-3 PSM estimate for ideal thermogram

\begin{tabular}{|c|c|c|c|c|c|c|c|c|}
\hline \multirow[b]{2}{*}{ Tissue Type } & \multicolumn{3}{|c|}{ Target parameter } & \multirow[b]{2}{*}{ Run } & \multicolumn{3}{|c|}{ Estimated parameter } & \multirow[b]{2}{*}{$\begin{array}{l}\text { Avg. } \\
\text { Error }\end{array}$} \\
\hline & $h(\mathrm{~cm})$ & $\begin{array}{c}Q_{m} \\
\left(\mathrm{~W} / \mathrm{m}^{3}\right)\end{array}$ & $\begin{array}{c}R \\
(\mathrm{~mm})\end{array}$ & & $h(\mathrm{~cm})$ & $\begin{array}{c}Q_{m} \\
\left(\mathrm{~W} / \mathrm{m}^{3}\right)\end{array}$ & $\begin{array}{c}R \\
(\mathrm{~mm})\end{array}$ & \\
\hline \multirow{3}{*}{ Chest } & \multirow{3}{*}{2.5} & \multirow{3}{*}{40000} & \multirow{3}{*}{9} & Set-1 & 2.4977 & 40221 & 9.0072 & \multirow{3}{*}{$0.55 \%$} \\
\hline & & & & Set-2 & 2.5007 & 40110 & 8.9896 & \\
\hline & & & & Set-3 & 2.5036 & 39963 & 9.0424 & \\
\hline \multirow{3}{*}{ Forearm } & \multirow{3}{*}{1.6} & \multirow{3}{*}{50000} & \multirow{3}{*}{12} & Set-1 & 1.5833 & 50360 & 12.0232 & \multirow{3}{*}{$1.04 \%$} \\
\hline & & & & Set-2 & 1.5950 & 50272 & 12.0584 & \\
\hline & & & & Set-3 & 1.6037 & 49832 & 11.9792 & \\
\hline \multirow{3}{*}{ Breast } & \multirow{3}{*}{1.2} & \multirow{3}{*}{65000} & \multirow{3}{*}{8} & Set-1 & 1.1964 & 65307 & 8.0312 & \multirow{3}{*}{$0.61 \%$} \\
\hline & & & & Set-2 & 1.2081 & 64868 & 8.04 & \\
\hline & & & & Set-3 & 1.2110 & 64604 & 7.9784 & \\
\hline
\end{tabular}

Table 6-4 PSM estimate for realistic thermogram

\begin{tabular}{|c|c|c|c|c|c|c|c|c|}
\hline \multirow{2}{*}{$\begin{array}{l}\text { Tissue } \\
\text { Type }\end{array}$} & \multicolumn{3}{|c|}{ Target parameter } & \multirow[b]{2}{*}{ Run } & \multicolumn{3}{|c|}{ Estimated parameter } & \multirow{2}{*}{$\begin{array}{l}\text { Avg. } \\
\text { Error }\end{array}$} \\
\hline & $h(\mathrm{~cm})$ & $R(\mathrm{~mm})$ & $\begin{array}{c}Q_{m} \\
\left(\mathrm{~W} / \mathrm{m}^{3}\right)\end{array}$ & & $h(\mathrm{~cm})$ & $R(\mathrm{~mm})$ & $\begin{array}{c}Q_{m} \\
\left(\mathrm{~W} / \mathrm{m}^{3}\right)\end{array}$ & \\
\hline \multirow{3}{*}{ Chest } & \multirow{3}{*}{2.5} & \multirow{3}{*}{40000} & \multirow{3}{*}{ 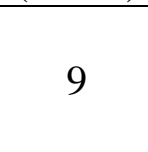 } & 1 & 2.4889 & 39984 & 8.9632 & \multirow{3}{*}{$0.96 \%$} \\
\hline & & & & 2 & 2.5095 & 40335 & 8.9281 & \\
\hline & & & & 3 & 2.5211 & 40247 & 9.0863 & \\
\hline \multirow{3}{*}{ Forearm } & \multirow{3}{*}{1.6} & \multirow{3}{*}{50000} & \multirow{3}{*}{12} & 1 & 1.6184 & 50359 & 11.9353 & \multirow{3}{*}{$1.15 \%$} \\
\hline & & & & 2 & 1.5891 & 49832 & 12.0672 & \\
\hline & & & & 3 & 1.6038 & 50184 & 12.1111 & \\
\hline \multirow{3}{*}{ Breast } & \multirow{3}{*}{1.2} & \multirow{3}{*}{65000} & \multirow{3}{*}{8} & 1 & 1.1905 & 64780 & 7.9257 & \multirow{3}{*}{$1.65 \%$} \\
\hline & & & & 2 & 1.2198 & 65307 & 8.0663 & \\
\hline & & & & 3 & 1.2139 & 65220 & 8.0487 & \\
\hline
\end{tabular}

\subsubsection{Genetic Algorithm}

Genetic algorithm (GA), a search heuristic that is developed by mimicking the concept of natural selection, was formally introduced in the United States in the 1970s by John Holland at the University of Michigan and still is routinely used for generating useful solutions to optimization and search problems and the continuing price/performance improvement of computational systems have compelled ample attraction for non-gradient optimization [63]. The process uses natural evolution, such as inheritance, mutation, selection and crossover for generating solutions to optimization problems. The GA, however computationally expensive, is less susceptible to getting 'stuck' at local optima than gradient search methods and works very well on mixed (continuous and discrete), combinatorial problems, in particular.

Features of the Genetic Algorithm: The most important features of GAs are, 
- definition of the objective/cost/fitness function;

- definition and implementation of the genetic representation (chromosome); and

- definition and implementation of the genetic operators.

Fitness function: The fitness function is used to achieve the optimized solution by minimizing $\min f(x)$ where $f: \mathbb{R}^{n} \rightarrow \mathbb{R}, x \in \mathbb{R}^{n}$ and $x$ is called the chromosome. Thecost function is evaluated foreach chromosome and depending on the cost value, the processassigns a figure of merit to indicate how close it for meeting the overall specification, and then the worst solutions (chromosomes) are deleted and the good ones breed to form new chromosomes until the best design solutions emerge. The essential features of a fitness function would be workable, closely relatable and quickly computable. The cost function is developed involving the simulated pattern (obtained from the numerical solution of bio-thermal problem over the anatomic accurate mode) and IR thermogram (test or target pattern, obtained from clinic taking thermogram over the surface of breast with tumor).

Genetic Representation: The process operates on twenty populations, encoded in $40 \mathrm{bit}$ binary, called the chromosome or genome. The five genes (parameters) on each chromosome are the depth $h$, size $R$ (radius), blood perfusion rate $\omega_{b}$, and metabolism $Q_{m}$ (each parameter is 10 bit long). The genetic algorithm creates a population of genomes (initial populations or candidate solutions) and then it applies genetic operations (selection, crossover and mutation), iteratively, to the individuals in the population to generate new individuals. To pick up the best individuals for mating, the process evaluates the cost function and assigns an objective score. The objective scores help the genetic algorithm to maintain diversity. The transformation from the raw objective scores to the scaled fitness scores is obtained by the linear scaling (fitness score = objective score/overall variation in scores. The process will use the 'steady state' implementation i.e. at each 'generation' a few individuals are replaced with the good ancestors. The worst and the most-similar chromosomes are to be replaced.

Genetic Operations: A probability percentage of mutation of 0.10 and a probability of crossover 0.40 are used to determine how members of a population will reproduce to bring forth the next generation. In the crossover, two individuals (the parents) combine to produce two more individuals (the children), thus the genetic material from the previous generation is carried to the subsequent generation (offspring). Chromosomes of higher fitness are more likely to be selected 
to reproduce and contribute their genetic material to the next generation. In the mutation process, the elementflip, element swap and destructive mutation are to be used, to add additional features to the candidate solution.

The GA based evolution process goes:

- Start with random population

- Evaluate fitness of each population

- Create new population

○ Select parents based on fitness

○ Create new generation through crossover and mutation of "DNA"

- Replace old population

- Repeat

Picking the best individual in producing new population makes the process quick converge. However, picking some genomes that aren't quite as good (but hopefully have some good genetic material in them) is a good practice. Some of the useful section methods are the roulette wheel selection (the likelihood of picking an individual is proportional to the individual's score), the tournament selection (a number of individuals are picked using roulette wheel selection, then the best of these is (are) chosen for mating), and the rank selection (pick the best individual every time). The threshold selection can also be effective.

The crossover and the selection methods, mentioned above, are too effective that they eventually will be driving the GA toward a population of individuals that are almost exactly the same. When the generations are ended up with a population that consists of similar individuals, the likelihood of finding new solutions typically decreases and the process stops.

\subsubsection{GA Process}

Like GPM and PSM optimization, the GA also needs to identify

- Ideal temperature distribution - thermal model of faulty (tumor) organ is analyzed numerically to estimate spatial distribution of temperature over surface. Assuming a heterogeneous organ model with known bio-thermal characters for healthy tissues while unknown physio-bio-thermal features for tumor, the surface temperature profile 
is estimated in a controlled laboratory condition. Therefore the spatial distribution of temperature $T_{\text {sim }}\left(\omega_{b}, h, Q_{m}, R\right)$.

- Parameter vector - the optimization parameters are the perfusion rate, depth, metabolism and radius of tumor. So the parameter vector is $b=\left[\omega_{b}, h, Q_{m}, R\right]$.

- Optimize function-the process will minimize $E=\sum_{i=1}^{n} \mid T_{I R}-$ $T_{s i m}\left(\omega_{b i}, h_{i}, Q_{m i}, R_{i}\right) \mid$.

Spatial distribution of surface temperature for an organ with a tumor will be acquired by thermogram and the center of the abnormal region and isothermal contours will be determined by modified snake algorithm [23] which will give test data set $\left(T_{I R}\right)$.

\begin{tabular}{c|c|c|c|}
\hline 1001110011 & 1101110111 & 1000110101 & 1011111010 \\
\hline Perfusion rate & Depth & Metabolic rate & Radius \\
Figure 6-10 & Representation of a chromosome
\end{tabular}

The vector $b$ is represented by a chromosome, commonly converted to a binary array. This study the binary array (chromosome) encodes four values in the order shown, after some execution the order has determined. In the implementation of the GA, the potential solution (chromosome) contains four genes named, respectively, as perfusion rate, depth, heat rate and radius and 10 bits are used to represent the value of each gene in binary format. Therefore, each chromosome has forty bits. This presentation is shown graphically in Figure 6-10. The fitness function is $f(b)=$ $\frac{1}{E^{2}(b)}$.

The population size is chosen to be fifty and initial population pool has been generated using a linearly spaced [ $1-2]$ random number generator. The fittest parents are chosen stochastically to keep the gene pool from getting trapped easily in a local max of the fitness function. The stochastic process must weigh the chromosomes by their fitness, calculate the sum of the fitness of all chromosomes, and select the parents represented by the value $r$ which is a random number between 1 and 50 (population size).

Accuracy of the GA system depends on the size of chromosome. The study has used $40 \mathrm{bit}$ chromosome where each parameter is presented in 10 bits. Before applying to the objective function evaluation, the binary parameters are to be converted to decimal numbers. The parameters are changed from their binary representation to floating point representation using: 


$$
\Gamma_{\mathrm{n}}=1+\frac{1}{2^{\mathrm{x}}-1} \mathrm{D}
$$

where, $x$ is the number of bits used to represent the parameter (e. g. 10 in this case), and D is the decimal value of the parameter in binary form. The step size (resolution) of normalized parameters is $\Delta \Gamma_{\mathrm{n}}=\left(2^{\mathrm{x}}-1\right)^{-1}$. For example a 6-bit parameter, as shown in the Table, the normalized parameters have a step size of 0.0159 which provides an actual stepping of $4.77 \times$ $10^{-4} \mathrm{~m}, \quad 4.69 \times 10^{-2} \mathrm{~K}$ in and $1.406 \times 10^{-4} \mathrm{~m}$ in the estimation of depth, tumor center temperature and radius, respectively.

The initial populations are basically based on normalized parameters which are generated using 'rand' function of MATLAB and holding them between 1.1 and 1.9. Assume 20 initial populations and repeat the following steps for each of them. Decode the randomly chosen population to produce an actual thermo-physical parameter which is then fed to the numerical model to compute surface temperature profile. Identify the abnormal features of the temperature profile and mark some isothermal lines using the modified snake algorithm. Compare the contour with the test pattern and evaluate the fitness of that particular chromosome using (6.22) and then ranked. Chromosomes of higher fitness are more likely to be selected to reproduce and contribute their genetic material to the next generation. A probability of mutation of 0.10 and a probability of crossover 0.40 are used to determine how members of a population will reproduce to bring forth the next generation. The crossover process takes place according the following approaches:

Intra segment crossing: In this approach one or more bits of a segment will be interchanged with the respective bit(s) of the similar segment between two parents having the closest fitness error.

Inter-segments crossing: In this approach one or more bits of a segment will be interchanged with the respective bit(s) of the different segment between two parents having the closest fitness error.

The fitness of each chromosome is evaluated using Eqn. (6.22) and then ranked. Chromosomes of higher fitness are more likely to be selected to reproduce and contribute their genetic material to the next generation. A probability of mutation of 0.10 and a probability of crossover 0.40 are used to determine how members of population will reproduce to bring forth the next generation. In detail, the process has carried out according the flow chart presented in Figure 
6-11. The process requires initialization of the set of bio-thermal parameters listed in Table 3.2, and Table 5.1 through 5.7. Among the listed parameters, healthy tissue's bio-thermal features (such as $k, c_{b}, \rho_{b}, \omega_{b}, Q_{m}, T_{a}$ ) and ambient features (such as $h_{a}, T_{e}$ ) are kept unaffected while the tumor parameters, named as solution vector ( includes $k, \omega_{b}, Q_{m}, h, R$ ), keep changing during the process. Initial solution vector, the starting point of the search, can be chosen randomly using random function generator or using Eqn. 6.13. The solution vector is repeatedly computed by a search algorithm tailored using PSM, GA or GPM. In a particular iteration the estimated solution vector and other bio-thermal parameter values are applied to simulate the surface thermal distribution over any the anatomic accurate model developed in Chapter 4 (see Figure 4.6, 4.8, 4.10-4.12). The bio-heat flow problem can be solved analytically or numerically (using FDM or FEM) or utilizing the heat-source mode (see section 6.3.1.1). However, the estimated surface thermal image can also be obtained by using a trained neural network.

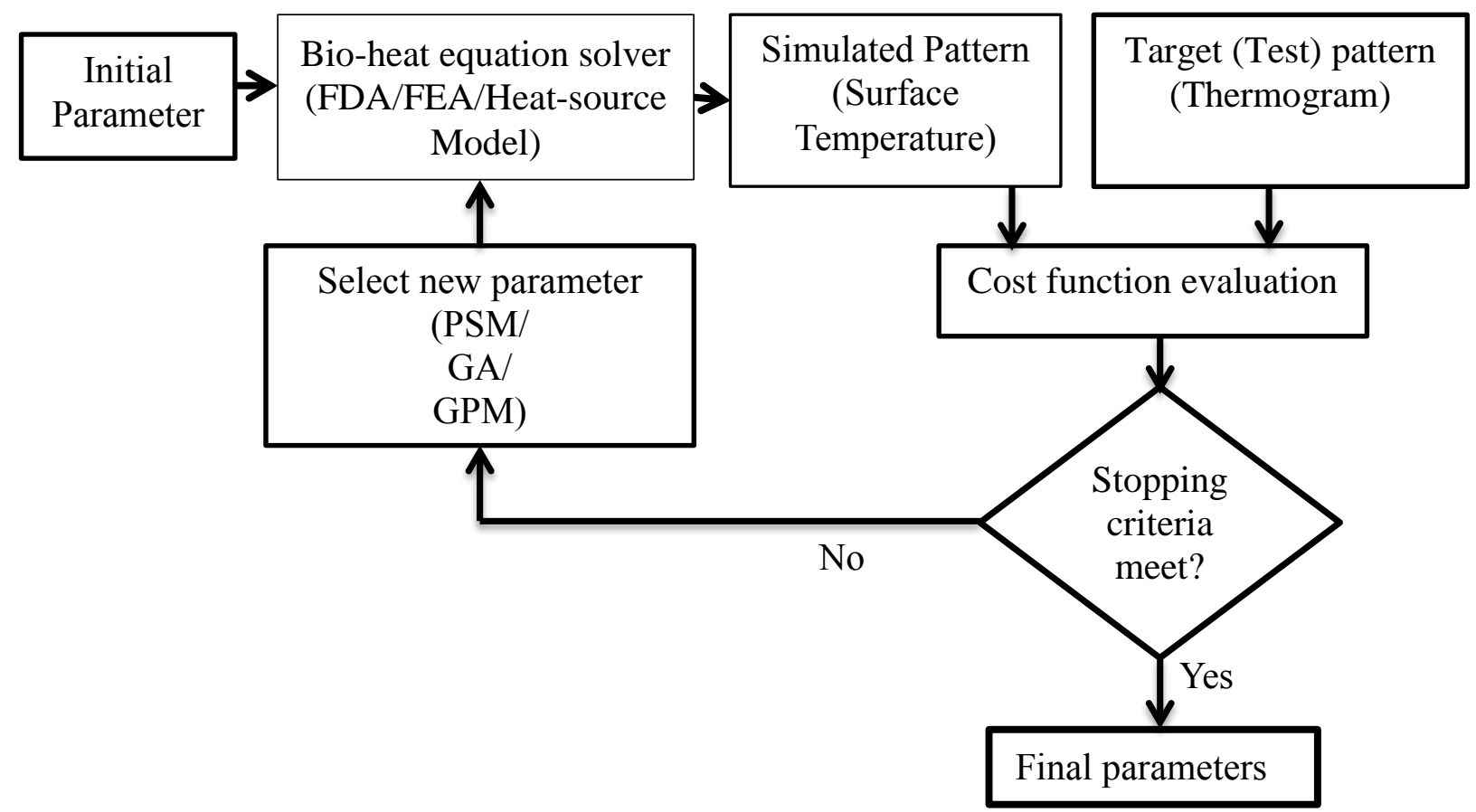

Figure 6-11 GA based estimation procedure

The simulated surface thermal pattern is compared with a test (target) thermogram. The thermogram may be obtained from a clinic or generated in a laboratory. In this study, pretend thermal dataset has been generated by FEM simulation of bio-heat flow problem on an anatomicaccurate physical model in a noisy environment (a random noise up to $\pm 10 \%$ to be added with ideal data to develop pretend in-vivo dataset). Section 6.3.2 discusses the procedure of developing 
the test $\left(T_{I R}\left(k, Q_{m}, \omega_{b}, h, R, \bar{r}\right)\right)$ and simulated $\left(T_{s i m}\left(k, Q_{m}, \omega_{b}, h, R, \bar{r}\right)\right)$ datasets from the respective thermograms, where $\bar{r}$ is the space variable. The optimization process advances while minimizing the mean absolute difference between temperatures at some predefined points (Eqn. 6.14) and minimizing the mean absolute difference between the distances from the warmest point to the isothermal contours (Eqn. 6.15). If both cost functions achieve minimum values than the previous iteration, the solution vector set for that particular iteration is said to be converge to an optimum point and the search becomes a successful search. The searching method is repeated until any stopping criteria meets to terminate the process which are-(i) the optimization error should be $\geq 10^{-10}$ and (ii) the maximum iteration ( $N=1000$ for GPM and ANN and $N=30$ Generations for GA). If none of this stopping condition meets, the process is repeated for new solution vector.

\section{The algorithm}

1) Generate initial population $X_{0}=\left\{x_{1}^{0}, x_{2}^{0}, \ldots \ldots \ldots, x_{N}^{0}\right\}, x_{i}^{0} \in \Omega$ and $\mathrm{N}$ is even

2) Evaluate cost function $\varepsilon_{0}^{*}=\sum_{j=1}^{n}\left|T_{a c t u a l, j}-T_{j}\left(x_{i}^{N}\right)\right|, j \in \mathbb{R}$

3) Record $S \subset\left\{\left(\varepsilon_{0}^{1}, x_{1}^{0}\right),\left(\varepsilon_{0}^{2}, x_{2}^{0}\right), \ldots \ldots \ldots \ldots,\left(\varepsilon_{0}^{N}, x_{N}^{0}\right)\right\}$ and sort in ascending

4) Repeat $t=0,1, \ldots \ldots \ldots$

a) Repeat $\mathrm{Row}=1,3,5 \ldots \ldots, \mathrm{N} / 2$

i) Parent selection $[\bar{X}, \bar{Y}]=\operatorname{select}\left(S_{\text {Row:Row }+1} ; S_{\text {Col2:4 }}\right)$

ii) Repeat Bit $=2,3, \ldots$,Length-Bit +1

1. $\hat{x}=$ crossover $[\bar{X}($ Bit $), \bar{Y}($ Bit $)]$

2. Evaluate cost function and add to $\mathrm{S}$.

iii) Repeat NBits $=1, \ldots \ldots$,Length-2

1. Parent selection $[\bar{X}, \bar{Y}]=\operatorname{select}\left(S_{\text {Row:Row }+1} ; S_{\text {Col2:4 }}\right)$

2. $\hat{x}=$ crossover $[\bar{X}($ Bit $), \bar{Y}($ Bit $)]$

3. Evaluate cost function and add to $\mathrm{S}$.

iv) Repeat Bit $=2,3, \ldots$,Length-Bit +1

1. $\hat{x}=$ crossover $[\bar{X}($ Bit $), \bar{Y}($ Bit + Length $)]$ 
2. Evaluate cost function and add to $\mathrm{S}$.

v) Repeat step 4(a-iii) with replacing (iii-2) by (iv-1).

b) Repeat Row $=1,2,3 \ldots \ldots, \mathrm{N}$

i) Parent selection $[\bar{X}]=\operatorname{select}\left(S_{\text {Row }} ; S_{\text {Col2:4 }}\right)$

ii) For each segment repeat for $\mathrm{Bit}=2$, and 3

1. $\hat{x}=[\bar{X}(2: 2+B i t), \bar{X}(B i t+4: 2 * B i t+4)]$

2. Evaluate cost function and add to $\mathrm{S}$.

iii) Parent selection $[\bar{X}]=\operatorname{select}\left(S_{\text {Row }} ; S_{\text {Col2:4 }}\right)$

1. Repeat $\mathrm{Bit}=2,3, \ldots$ Length

2. $\hat{x}=[\bar{X}(2: 2+B i t), \bar{X}(2+$ Length: Bit $+2+$ Length $)]$

3. Evaluate cost function and add to $\mathrm{S}$.

5) Until some stopping criterion is satisfied

The subroutine developed in MATLAB, is currently performs a set number of iterations.

\subsubsection{Results}

The results obtained using these methodologies for solving the inverse problem related with tumors located in different parts of human body are listed in

Table 6-5 and Table 6-6. The parameters estimated from ideal surface temperature data of different tissue types are presented in the former table. For each cases the GA was run thrice with different random initial population, but the final estimates were identical regardless the initial population. These results show a good agreement between the actual and forecasted parameters, with maximum absolute error in heat generation rate of only $1.04 \%$ for the tumor embedded in rectangular pyramidal tissues. Moreover, when a random noise with maximum $\pm 10 \%$ was added to the input data the results obtained are in good agreement between the actual and forecasted parameters as presented in Table 6-6. In all cases the values of the known parameters and dimensions of the domain were same as presented in Chapter 1.

It can be seen that using the proposed methodology it is possible to determine the depth, radius, perfusion rate and heat generation rate of tumor with a good accuracy. 
Table 6-5 GA estimate for ideal thermogram of tumor buried in natural breast

\begin{tabular}{|c|c|c|c|c|c|c|c|c|c|}
\hline \multirow{2}{*}{$\begin{array}{l}\text { Tumor } \\
\text { Type }\end{array}$} & \multirow[b]{2}{*}{ Position } & \multicolumn{3}{|c|}{ Target Parameter } & \multirow[b]{2}{*}{ Run } & \multicolumn{3}{|c|}{ Simulated Parameter } & \multirow{2}{*}{$\begin{array}{c}\% \\
\text { Error } \\
\text { (Avg.) }\end{array}$} \\
\hline & & $\begin{array}{c}h \\
(\mathrm{~cm})\end{array}$ & $\begin{array}{c}R \\
(\mathrm{~mm}) \\
\end{array}$ & $\begin{array}{c}Q_{m} \\
\left(\mathrm{~W} / \mathrm{m}^{3}\right) \\
\end{array}$ & & $h(\mathrm{~cm})$ & $R(\mathrm{~mm})$ & $\begin{array}{c}Q_{m} \\
\left(\mathrm{~W} / \mathrm{m}^{3}\right)\end{array}$ & \\
\hline \multirow{3}{*}{ Benign } & \multirow{3}{*}{$\begin{array}{l}\text { Shallow } \\
\text { (on-axis) }\end{array}$} & \multirow{3}{*}{1.5} & \multirow{3}{*}{9} & \multirow{3}{*}{40000} & 1 & 1.4545 & 9.1738 & 38862.3 & \multirow{3}{*}{4.04} \\
\hline & & & & & 2 & 1.5375 & 8.6367 & 41181.64 & \\
\hline & & & & & 3 & 1.5228 & 9.2224 & 40439.45 & \\
\hline \multirow{3}{*}{$\begin{array}{c}\text { Maligna } \\
\text { nt }\end{array}$} & \multirow{3}{*}{$\begin{array}{l}\text { Shallow } \\
\text { (on-axis) }\end{array}$} & \multirow{3}{*}{1.8} & \multirow{3}{*}{15} & \multirow{3}{*}{80000} & 1 & 1.7767 & 14.7402 & 78754.9 & \multirow{3}{*}{2.17} \\
\hline & & & & & 2 & 1.8353 & 15.2283 & 81074.0 & \\
\hline & & & & & 3 & 1.8207 & 15.3262 & 80517.6 & \\
\hline \multirow{3}{*}{$\begin{array}{c}\text { Maligna } \\
\text { nt }\end{array}$} & \multirow{3}{*}{$\begin{array}{c}\text { Deep- } \\
\text { seated } \\
\text { (on-axis) }\end{array}$} & \multirow{3}{*}{2.5} & \multirow{3}{*}{21} & \multirow{3}{*}{90000} & 1 & 2.4408 & 21.2832 & 87846.68 & \multirow{3}{*}{3.21} \\
\hline & & & & & 2 & 2.5189 & 20.6484 & 90815.43 & \\
\hline & & & & & 3 & 2.5580 & 21.6738 & 92670.9 & \\
\hline \multirow{3}{*}{$\begin{array}{c}\text { Maligna } \\
\text { nt }\end{array}$} & \multirow{3}{*}{$\begin{array}{c}\text { Shallow } \\
\left(15^{\circ} \text { Off- }\right. \\
\text { axis })\end{array}$} & \multirow{3}{*}{2.0} & \multirow{3}{*}{15} & \multirow{3}{*}{75000} & 1 & 2.0551 & 15.2772 & 76806.64 & \multirow{3}{*}{3.03} \\
\hline & & & & & 2 & 2.0404 & 14.7400 & 75322.27 & \\
\hline & & & & & 3 & 1.9574 & 14.5452 & 72724.6 & \\
\hline
\end{tabular}

Table 6-6 GA estimate for realistic thermogram of tumor buried in deformed breast

\begin{tabular}{|c|c|c|c|c|c|c|c|c|c|}
\hline \multirow{2}{*}{$\begin{array}{l}\text { Tumor } \\
\text { Type }\end{array}$} & \multirow[b]{2}{*}{ Position } & \multicolumn{3}{|c|}{ Target Parameter } & \multirow[b]{2}{*}{ Run } & \multicolumn{3}{|c|}{ Simulated Parameter } & \multirow{2}{*}{$\begin{array}{c}\% \\
\text { Error } \\
\text { (Avg.) }\end{array}$} \\
\hline & & $\begin{array}{c}h \\
(\mathrm{~cm})\end{array}$ & $\begin{array}{c}R \\
(\mathrm{~mm})\end{array}$ & $\begin{array}{c}Q_{m} \\
\left(\mathrm{~W} / \mathrm{m}^{3}\right)\end{array}$ & & $h(\mathrm{~cm})$ & $\begin{array}{c}R \\
(\mathrm{~mm})\end{array}$ & $\begin{array}{c}Q_{m} \\
\left(\mathrm{~W} / \mathrm{m}^{3}\right)\end{array}$ & \\
\hline \multirow{3}{*}{ Malignant } & \multirow{3}{*}{ Shallow } & \multirow{3}{*}{1.5} & \multirow{3}{*}{12} & \multirow{3}{*}{75000} & 1 & 1.5424 & 12.250 & 73188.48 & \multirow{3}{*}{2.83} \\
\hline & & & & & 2 & 1.4789 & 11.859 & 74580.08 & \\
\hline & & & & & 3 & 1.4593 & 11.712 & 76435.55 & \\
\hline \multirow{3}{*}{ Malignant } & \multirow{3}{*}{$\begin{array}{l}\text { Deep- } \\
\text { seated }\end{array}$} & \multirow{3}{*}{2.5} & \multirow{3}{*}{21} & \multirow{3}{*}{90000} & 1 & 2.5824 & 19.330 & 91093.13 & \multirow{3}{*}{4.07} \\
\hline & & & & & 2 & 2.4570 & 20.599 & 8793.45 & \\
\hline & & & & & 3 & 2.6019 & 20.453 & 93041.99 & \\
\hline \multirow{3}{*}{ Malignant } & \multirow{3}{*}{$\begin{array}{c}\text { Off- } \\
\operatorname{axis}\left(10^{0}\right)\end{array}$} & \multirow{3}{*}{2.0} & \multirow{3}{*}{18} & \multirow{3}{*}{60000} & 1 & 1.9672 & 18.744 & 57973.63 & \multirow{3}{*}{4.13} \\
\hline & & & & & 2 & 2.0746 & 18.451 & 61220.70 & \\
\hline & & & & & 3 & 2.0404 & 17.621 & 62333.98 & \\
\hline
\end{tabular}

Genetic Algorithms are not chaotic, they are stochastic. The complexity depends on the genetic operators, their implementation (which may have a very significant effect on overall complexity), the representation of the individuals and the population, and obviously on the fitness function. Given the usual choices (point mutation, one point crossover, and roulette wheel selection) a Genetic Algorithms complexity is $O\{g(n m+n m+n)\}$ with $g$ the number of generations, $n$ the population size and $m$ the size of the individuals. Therefore the complexity is on the order of $O(\mathrm{gnm})$. In this particular algorithm, $g=20, n=20$ and $m=30$. This is of 
cause ignoring the fitness function, which depends on the application, in this case, the complexity is on the order of $O(n)$.

\subsubsection{Artificial Neural Network}

The capability of machine learning and pattern recognition by animals' central nervous systems are mimicked in developing computational models named Artificial Neural Network (ANN). ANNs are usually presented as systems of interconnected "neurons" that can compute values from inputs by feeding information through the network. The set of highly interconnected processing elements (neurons) are labelled with integers $1,2, \ldots \ldots, N$. They are connected through a set of connection weights, or synaptic weights. Every neuron $i$ has $M_{i}$ inputs, and one output $y_{i}$. The inputs are real valued quantities labeled $s_{i 1}, s_{i 2}, \ldots \ldots, s_{i M i}$, representing signals coming either from other neurons in the network, or from the external world. Every neuron $i$ has $M_{i}$ synaptic weights, each one associated with each of the neuron inputs. The synaptic weights are labelled as $w_{i 1}, w_{i 2}, \ldots \ldots, w_{i M i}$, and represent real valued quantities that multiply the corresponding input signal. Every neuron $i$ has also an extra input, which is set to a fixed value $I_{i}$, and is referred to as the threshold of the neuron. Every neuron computes its own internal state, or total activation, according to the following expression:

$$
x_{i}=\sum_{j=1}^{M_{i}} w_{i j} s_{i j}+I_{i}=\sum_{j=0}^{M_{i}} w_{i j} s_{i j}
$$

Every neuron $i$ computes its output according to a function $y_{i}=f\left(x_{i}\right)$, where $f$ is the neuron function or transfer function.

There are many types of neural network available now, for example, the Feed-forward neural network, the Radial basis function (RBF) network, the Kohonen self-organizing network Learning Vector Quantization, Fully recurrent network (Hopfield network, Boltzmann machine), Simple recurrent networks, Echo state network,_Long short term memory network, Bi-directional RNN, Hierarchical RNN, Stochastic neural networks, Modular neural networks etc.

Learning in a neural network is called training. Like training in athletics, training in a neural network requires a coach, someone that describes to the neural network what it should have produced as a response. From the difference between the desired response and the actual response, the error is determined and a portion of it is propagated backward through the network. At each 
neuron in the network the error is used to adjust the weights and threshold values of the neuron, so that the next time, the error in the network response will be less for the same inputs.

This corrective procedure is called backpropagation (hence the name of the neural network) and it is applied continuously and repetitively for each set of inputs and corresponding set of outputs produced in response to the inputs. This procedure continues so long as the individual or total errors in the responses exceed a specified level or until there are no measurable errors. After the neural network has learned the training material, the training process should be stopped and the trained network can be used network to produce responses to new input data (Figure 6-12).

Backpropagation starts at the output layer with the following equations:

$$
w_{i j}=W_{i j}^{\prime}+L R \cdot e_{j} \cdot X_{i}
$$

and

$$
e_{j}=Y_{j} \cdot\left(1-Y_{j}\right) \cdot\left(d_{j}-Y_{j}\right)
$$

For the $\mathrm{i}^{\text {th }}$ input of the $\mathrm{j}^{\text {th }}$ neuron in the output layer, the weight $w_{i j}$ is adjusted by adding to the previous weight value, $W_{i j}^{\prime}$, a term determined by the product of a learning rate, $\boldsymbol{L} \boldsymbol{R}$, an error term, $e_{j}$, and the value of the $\mathrm{i}^{\text {th }}$ input, $X_{i}$. The error term, $e_{j}$, for the $\mathrm{j}^{\text {th }}$ neuron is determined by the product of the actual output, $Y_{j}$, its complement, $\left(1-Y_{j}\right)$, and the difference between the desired output, $d_{j}$, and the actual output.

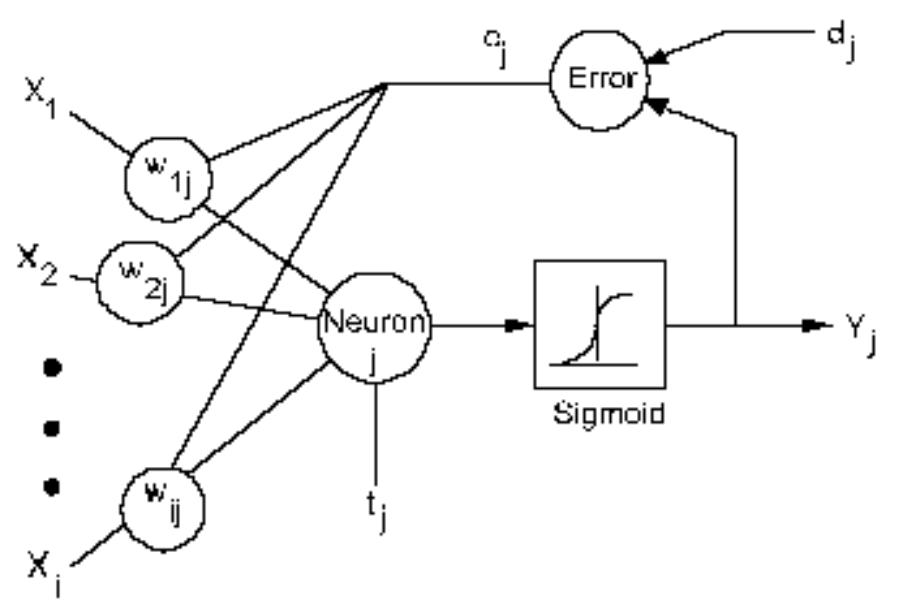

Figure 6-12 Neuron weight adjustment 
Once the error terms are computed and weights are adjusted for the output layer, the values are recorded and the next layer back is adjusted. The same weight adjustment process, determined by Eqn. 6.1, is followed, but the error term is generated by a slightly modified version of Eqn. 6.2. This modification is:

$$
e_{j}=Y_{j} \cdot\left(1-Y_{j}\right) \cdot \sum\left(e_{k} \cdot w_{j k}^{\prime}\right)
$$

The difference between the desired output and the actual output is replaced by the sum of the error terms for each neuron, $k$, in the layer immediately succeeding the layer being processed (remember, we are going backwards through the layers so these terms have already been computed) times the respective pre-adjustment weights.

The learning rate, $\boldsymbol{L R}$, applies a greater or lesser portion of the respective adjustment to the old weight. If the factor is set to a large value, then the neural network may learn more quickly, but if there is a large variability in the input set then the network may not learn very well or at all. In real terms, setting the learning rate to a large value is analogous to giving a child a spanking, but that is inappropriate and counter-productive to learning if the offense is as simple as forgetting to tie their shoelaces. Usually, it is better to set the factor to a small value and edge it upward if the learning rate seems slow. In many cases, it is useful to use a revised weight adjustment process. This is described by the equation:

$$
w_{i j}=w_{i j}^{\prime}+(1-M) \cdot L R \cdot e_{j} \cdot X_{j}+\cdot\left(w_{i j}^{\prime}-w_{i j}^{\prime \prime}\right)
$$

This is similar to Eqn. 6.1, with a momentum factor, $\boldsymbol{M}$, the previous weight, $w_{i j}^{\prime}$, and the next to previous weight, $w_{i j}^{\prime \prime}$, included in the last term. This extra term allows for momentum in weight adjustment. Momentum basically allows a change to the weights to persist for a number of adjustment cycles. The magnitude of the persistence is controlled by the momentum factor. If the momentum factor is set to 0, then the equation reduces to that of Eqn. 6.1. If the momentum factor is increased from 0 , then increasingly greater persistence of previous adjustments is allowed in modifying the current adjustment. This can improve the learning rate in some situations, by helping to smooth out unusual conditions in the training set.

For the trained network the total error, that is the sum of the errors over all the training sets, will become smaller and smaller. Once the network reduces the total error to the limit set, training may stop. Then apply the network, using the weights and thresholds as trained. 
It is a good idea to set aside some subset of all the inputs available and reserve them for testing the trained network. By comparing the output of a trained network on these test sets to the outputs you know to be correct, you can gain greater confidence in the validity of the training. If you are satisfied at this point, then the neural network is ready for running.

Usually, no backpropagation takes place in this running mode as was done in the training mode. This is because there is often no way to be immediately certain of the desired response. If there were, there would be no need for the processing capabilities of the neural network! Instead, as the validity of the neural network outputs or predictions are verified or contradicted over time, you will either be satisfied with the existing performance or determine a need for new training. In this case, the additional input sets collected since the last training session may be used to extend and improve the training data.

\subsubsection{ANN Process}

The study has developed a 3-layer feed-forward ANN with back propagation learning to estimate thermo-physical parameters of tumors from surface thermal images. The network is developed with the MATLAB Neural Network Toolbox using the "newff" function. The "transig" transfer function was chosen for the hidden layers, "purelin" for the output layer and "trainlm" for back propagation training.

The ANN is trained with idealized surface temperature profiles obtained for 5 different cases. Set of 500 data from the half of the symmetric pattern is normalized and then taken as training/validating input, $T$ (1 $\times 500$ matrix). The target matrix $P$ is also normalized and each parameter is encoded with random numbers to produce 500 different values and scaled down to produce a $4 \times 500$ matrix. The learned network is then simulated for the above sample temperature pattern. The simulated output is, finally, decoded and justified with the constraints in Eqn. 6.15 to filter out the noisy data and the algebraic mean of the remaining data is computed and treated as the output of that run. The forecasted parameter's values are applied to the developed heat source model to produce the temperature pattern. If such obtained temperature patter is identical to the actual pattern then the run is considered as good run. In the end, the average of 20 good runs is computed to forecast the tumor parameters.

The heat source model was simulated with different parameter values and the simulated result was used for training of the ANN. In the proposed model, the surface (skin) is considered as 
a set of concentric isothermal circles where the surface temperature decreases from the centre to the perimeter. Assume the centre of the concentric circles in origin $(0,0)$ and evaluate the temperature of the isothermal circles at regular interval.

\subsubsection{Results}

Infrared scanning camera acquires the spatial distribution of surface temperature which is sampled and quantized to develop a two dimensional array (Ai, Ti) and fed to the developed ANN. The network is trained with the theoretical data, assuming $\mathrm{Ai}$ is the input vector (location) and $\mathrm{Ti}$ is the target vector (temperature at that location). After learning, the trained network is then simulated for a random test vector $(\mathrm{A}, \mathrm{T})$. The plot of the simulated data will be compared with the actual temperature image obtained by IR camera. A pattern classification model will be developed in the next phase of the research for comparison.

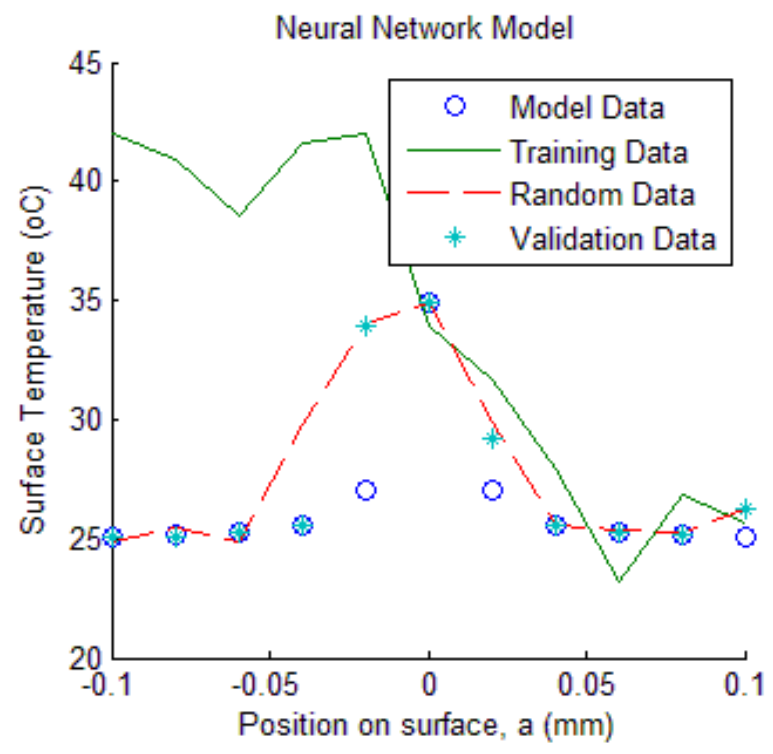

Figure 6-13 Temperature distribution

The output of the created neural network is shown in Figure 6-13 which shows four curves; the model data curve is the plot of theoretical model, the train data curve is the plot of the trained network with theoretical data, the validation curve is the performance indication of the trained network with the same test vector used for the training of the network, and finally the simulation curve with random data where the input data vector is obtained from data acquisition system. 


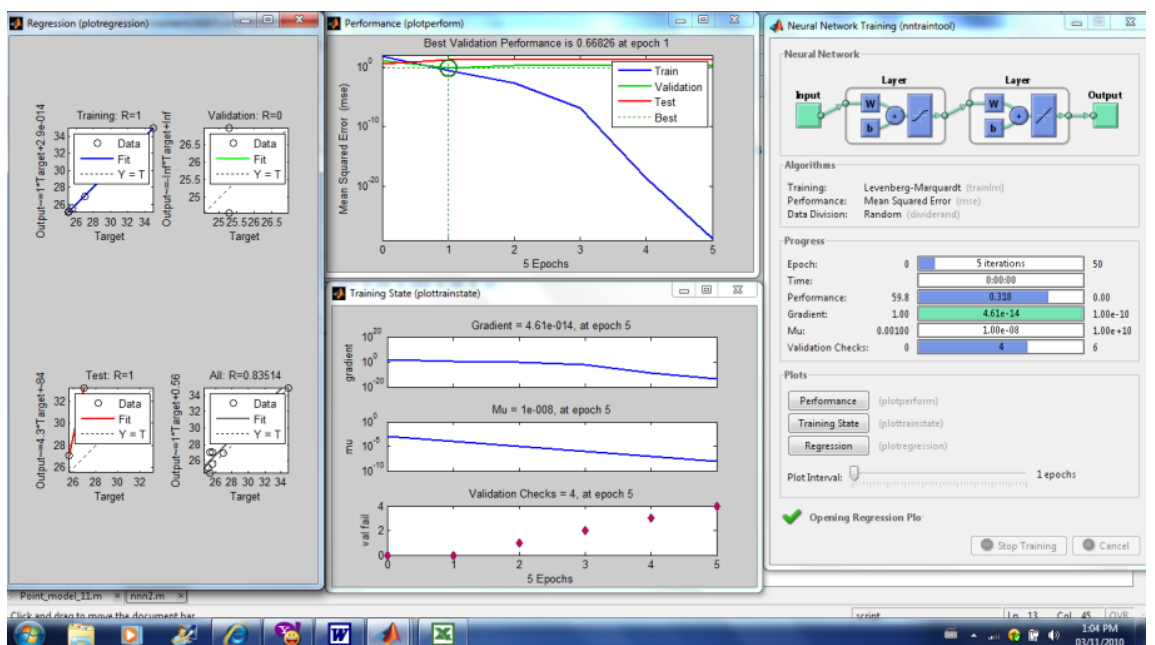

Figure 6-14 Neural network performance

The Levenberg-Marguardt algorithm has been used for network training and the mean square error for performance analysis. From Figure 6-14 it is seen that the trained network can reproduce 8 values out of 11 samples.

The ANN simulated temperature plot with random input vector will be compared with the actual temperature image captured by IR camera and the mismatch error is feedback to the system to recalculate the weight as long as the tumor parameters especially the thermal conductivity, metabolic rate, location and size of the hyperactive nodule is determined. The IR camera is capable to acquire the temperature at different points of a target surface and store in a data file that could be considered as a two dimensional array $(X, T)$, where $X(x, y)$ is the location on surface and $T$ is the temperature at that point. The distance ' $a$ ' is measured from the highest temperature point to $\mathrm{X}(\mathrm{x}, \mathrm{y})$.

For this purpose three different neural networks have been created and trained with the theoretical data obtained for point, disc and spherical heat source respectively. The trained network is simulated with the distance vector to get temperature as output vector.

Let us assume the unknown heat source is point shape. The test vector set is fed to the 'point source trained network'. The trained and simulated plots of point heat source are shown in Figure 6-15. The plots show that the simulated plot (* line) is quite different from sample plot (-- line). Again, assume the heat source is spherical in shape. The simulated, trained and sample plots, Figure 6-16, show that the graphs are identical in shape. It concludes that the unknown heat source is spherical in shape. 


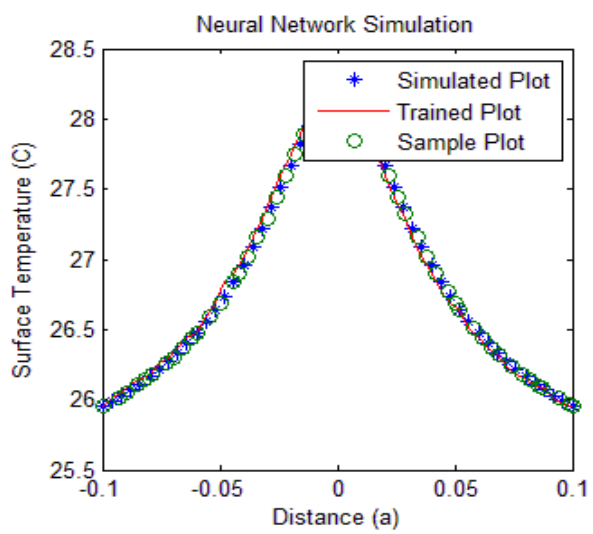

Figure 6-15 Best Simulated patterns

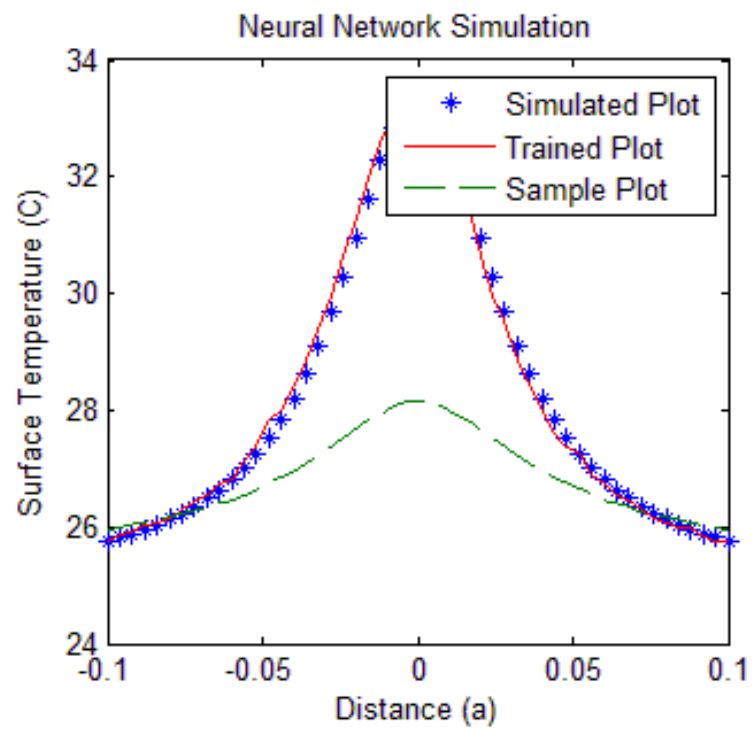

Figure 6-16 Simulated pattern (not the best one)

\subsection{Summary}

A methodology was developed for the estimation of thermo-physical or geometrical parameters of tumor region using the temperature profile on the skin surface that may be obtained by infrared thermography. The problem was solved using finite element method for physical model of tissues which have different geometry. Then the inverse problem was solved using genetic algorithm to estimate thermal and physical parameters of tumor which could grow in any organ of human body. The presented results demonstrate the feasibility of the proposed methodology. Even in case of $\pm 10 \%$ (maximum) noisy system the methodology estimates different parameters with a very good accuracy. 
According to the obtained results, the methodology can help to locate tumor region on any external body part, which could be useful and important to study tumor evolution after a treatment procedure. 


\section{Chapter 7 General Conclusions and Future Work}

\subsection{Conclusions}

The objective of the work presented in this thesis was to develop a methodology for diagnosis and parametrization of tumors embedded in a human organ from the abnormal surface temperature distribution that was acquired by thermography. Due to the extreme roughness and

high incidence of false positive outcome of thermogram interpretation, its clinical application was limited only to cancer detection. Improved performance in earlier detection of cancer was observed when thermal images were applied in combination with numerical simulation.

The developed methodology has tailored "an inverse problem solver" for the estimation of thermo-physical or geometrical parameters of a tumor region. The solver has coupled numerical solution of a second order differential equation, the Penne's bio-heat model, with a custom-made optimization algorithm. Comprehensive three dimensional thermal models of living organs, termed as "anatomic accurate models", have been developed to accurately understand and determine the influences of tumor parameters on the surface temperature distributions. The study has developed physical models for the chest, forearm, breast and drooped breast because the underlying idea was to demonstrate how shape and tissue inhomogeneity influence the spatial temperature distribution. The cuboid model of chest is built on four layers - the body core, muscle, fat and skin where heat flows in the radial direction from the core toward the skin surface. The tubular model of forearm is comprised on radius, ulna, arteries, muscle, fat and skin layer and prominent heat flow takes place in the radial direction. The breast model is given a hemispherical shape overall with muscle, gland, fat, skin and areola tissues where axial heat flow takes place. Similar breast model was developed in previous studies, but that model did not consider the fat layer sandwiched between the muscle and the lobule layers. In this study, this fat layer has also mimicked in order to come up with accurate breast model. In addition, the chest and forearm models are new innovations.

The one-dimensional heat flow problem at steady state has been addressed analytically for cuboid, cylindrical and spherical living tissues. With the obtained analytical solutions, the effects of the thermal conductivity, the blood perfusion rate, the metabolic heat generation rate and the coefficient of heat transfer on the temperature distribution in living tissues are analyzed. The 
analytical solution has given a useful means to accurately study the temperature behavior of the biological system, and extended to validate the numerical result and temperature field reconstruction and hyperthermia tumor ablution. The main limitation of the analytical solution is that it has obtained on homogeneous tissues and is unfit to apply to comprehensive models. In addition, it is unable to compute surface temperature distribution.

The study has applied the finite element method to compute the thermal feature over the above mentioned physical models. Performance of the default analyzer has been compared with analytical solution and finite difference scheme developed in Matlab scripts and has found consistent with high accuracy. Eventually the library module has been accepted as a stand-alone tool for estimation of thermal features of living organs. The estimated thermal dataset are used to examine temperature distribution in tissue inside, over the skin surface, as well as to associate the temperature distribute with thermal-physical-biological parameters of a tumor embedded in the organs under study. Though tumor shapes and margins are widely varied, in this research work, only the spherical tumors with round margins have been modeled. To achieve high true positive earlier detection rate, the thermogram may be applied with numerical simulations.

Various inverse approaches have been developed in conjunction with thermography to determine how it may be applied to diagnose tumor in human body. Inputs into the indirect solvers, the "test datasets", are taken from the simulated thermal dataset enveloped with random noise up to $\pm 10 \%$. The "simulated datasets" have been acquired from the finite element analysis solver with the estimated parameter set where the parameter set has been determined by optimization algorithm. Search direction was defined by the directional derivative matrix in the gradient projection optimization where the matrix is formed and updated by a numerical solver. In pattern search optimization scheme the search space was defined by a pattern matrix where the matrix is a four dimensional array that holds single and simultaneous multi-parameter update. Except this predefined search algorithms, the research has applied evolutionary algorithm to search for new solution. Genetic algorithm has been tailored to estimate tumor parameters where each parameter is presented by 10 bit binary number and each chromosome is of 40 bits. Next solution was determined by single point cross-over and mutation with mutation probability set to $10 \%$. The fitness of each population was calculated by evaluating a cost function and ranked; the best fitted populations were undergone to mate. The cost function for all optimization algorithms was determined from the average absolute error of test and simulated datasets. The initial parameters 
were randomly selected with a random function generator. The optimized parameters were normalized between 1 and 2 and the scaling factor was determined by applied constraints, however, the constraints were not supported clinically. The estimated parameters computed by the genetic algorithm and pattern search algorithm were consistent; however, the parameters estimated from gradient projection method were erroneous because the gradient matrix was computed from the approximate heat source model. As the estimated parameters were not confirmed by clinical or surgical or other recognized method, the study has used artificial neural network (ANN) in supporting the accuracy of the research outcome. The networked was trained with realistic thermogram dataset and applied to estimate tumor parameters. The ANN was replaced the anatomic-accurate models developed in this study. Therefore, the ANN not only confirmed the estimate accuracy but also the appropriateness of the physical models.

The main observations of the research are:

- Development of anatomic-accurate models of living organs such as chest, forearm, breast and deformed breast by mimicking the organ's anatomy and shape.

- Solving bio-heat transfer problem analytically on the simplistic (homogeneous) models of the above mentioned organs to demonstrate how the shape (outlook) affects the tissue temperature and to explain why anatomic-accurate models are required.

- Solving bio-heat transfer problem numerically on the anatomic accurate models of the above mentioned organs in presence of tumors to show how tumor's physical, thermal and biological parameters influence the surface temperature. The rate of change in surface temperature with respect to a particular parameter (element of the directional derivatives) provides a useful means for inverse source computation from a temperature distribution.

- Numerically simulated thermogram with added noise, which was considered as a pretend clinical thermogram had been sampled to for creating target or test dataset.

- Gradient projection and pattern search methods are used to estimate tumor parameters. Evolutionary method such as genetic algorithm is also used to generate the new parameters. For each optimization methods, the initial parameters are randomly chosen. 
- The estimated results obtained from pattern search method and genetic algorithms were consistent. For higher accuracy, the genetic algorithm estimates are applied to the pattern search method.

- The final estimate was applied to a trained ANN to reproduce the thermal image. The absolute error between the reconstructed image and the test image was negligible, in the order of $0.005 \%$.

\subsection{Recommendation for Future Work}

The proposed methodology can estimate the parameters of a wide range of tumors accurately, however, for deep-seated benign tumors the estimate accuracy was observed to be less than $80 \%$ which can be resolved by active thermography.

The human body's individual behavior, age group, genetic effect, body mass index, personal habits are not taken into account in constructing the model, though they may have influences on the risk of having tumor or in doubling rate.

The outcome was not validated with clinical data or biopsy result; therefore, the methodology cannot be applied as a standalone diagnostic tool in detecting and diagnosis tumors. In order to validate the proposed methodology, the ongoing research in Electro-thermal laboratory at Ryerson University is trying to build collaboration with Canadian Breast Cancer Society to collect the surgical data and true thermogram. 


\section{Bibliography}

[1] "Canadian Breast Cancer Foundation," 2015. [Online]. Available: http://www.cancer.ca/en/cancer-information/cancer-type/breast/statistics/. [Accessed 01 May 2015].

[2] D. J. Schaefer, "Design of magnetic resonance systems," in Standard Handbook of Biomedical Engineering, New York, McGraw-Hill, 2003.

[3] J. G. Mottley, "Ultrasonic Imaging," in Biomedical Engineering Handbook, FL, CRC, Boca Raton, 1995.

[4] M. J. Yaffe, "Mammography," in Biomedical Engineering Handbook, FL, CRC, Boca Raton, 1995.

[5] R. B. Zipurasky, J. H. Meyer and N. P. Verhoeff, "PET and SPECT Imaging in Psychiatric Disorders," La Revue canadienne de psychiatrie, vol. 52, no. 3, pp. 55-62, 2000.

[6] N. A. Diakides and J. D. Bronzino, Medical Infrared Imaging, Florida: CRC, Boca Raton, 2007.

[7] P. C. Pieters, M. W. J and J. H. DeMeo, "Evaluation of the portal venous system: complementary roles of invasive and noninvasive imaging strategies," RadioGraphics, vol. 17, no. 1, pp. 879-895, 1997.

[8] J. F. Head, F. Wang, C. A. Lipari and R. L. Elliot, "The important role of infrared imaging in breast cancer," IEEE Eng. Med. Biol. Mag., vol. 19, no. 3, pp. 365-368, 2000.

[9] M. Miyakawa and J. C. Bolomey, Non-invasive thermometry of the human body, FL: CRC, Boca Raton, 1996.

[10] J. C. Chato, "Measurement of thermal properties of biological materials," in Heat Transfer in Medicine and Biology, New York, Plenum Press, 1985, pp. 167-173.

[11] H. F. Bowman, "Estimtion of tissue blood flow," in Heat Transfer in Medicine and Biology, New York, Plenum Press, 1985, pp. 193-230.

[12] R. N. Lawson, "Implication of surface temperatures in the diagnosis of breast cancer," Canadian Medical Association Journal, vol. 75, pp. 309-310, 1956.

[13] R. N. Lawson and M. S. Chugatai, "Breast cancer and body temperatures," Canadian Medical Association Journal, vol. 88, pp. 68-70, 1963. 
[14] J. P. Agnelli, A. A. Barrea and T. C. V, "Tumor location and parameter estimation by thermography," Mathematical and Computer Modelling, vol. 129, no. 1, pp. 1-4, 2010.

[15] M. Gautherie, "Thermography of breast cancer: measurement and analysis of IN-VIVO temperature and blood flow," Ann. N. Y. Acad. Sci., vol. 335, pp. 383-413, 1980.

[16] M. Mital and E. P. Scott, "Thermal detection of embedded tumors using infrared imaging," ASME journal of Biomechanical Engineering, vol. 129, no. 1, pp. 33-39, 2007.

[17] R. Warren, "Screening women at high risk of breast cancer on the basis of evidence," Eur. J. Radiol., vol. 39, no. 1, pp. 50-59, 2001.

[18] Z. Deng and L. J, "Mathematical modelling of temperature over skin surface and its implementation in thermal disease diagnostics," Computers in biology and medicine, vol. 34, pp. 495-521, 2004.

[19] G. A. Santa Cruz, J. Bertotti, S. Marin, S. J. Gonzalez, S. Gossio, D. Alvarez, B. M. C. Roth, P. Menendez, M. D. Pereira, M. Albero, L. Cubau, P. Orellano and S. J. Liberman, "Dynamic infrared imaging of cutaneous melanoma and normal skin in patients treated with BNCT," Applied radiation and isotopes, vol. 67, pp. 54-58, 2009.

[20] M. M. Chen, C. O. Pedersen and J. C. Chato, "On the feasibility of obtaining three dimensional information from thermographic measurements," ASME Journal of Biomechanical Engineering, vol. 99, pp. 58-64, 1977.

[21] H. Qi, P. T. Kuruganti and Z. Liu, "Early detection of breast cancer using thermal texture maps," IEEE proceedings, vol. 1, pp. 1-4, 2006.

[22] E. Y. K. Ng, "Is thermal scanner lossing its bite in mass screening of fever due to SARS?," Medical Physics, vol. 32, pp. 93-97, 2005.

[23] E. Y. K. Ng and G. J. L. Kaw, "IR scanner as fever monitoring devices: physics, physiology and clinical accuracy," in Biomedical Engineering Handbook, Florida, CRC Press, 2005, pp. $1-24$.

[24] M. L. Giger, N. Karssemeijer and S. G. Armato III, "Computer-aided diagnosis in medical imaging," IEEE Transaction on Medical Imaging, vol. 20, no. 12, pp. 1205-1208, 2001.

[25] E. Y. K. Ng and N. M. Sudharsan, "An improved three-dimensional direct numerical modeling and thermal analysis of a female breast with tumor," Intl. MechEngrs, vol. 215, pp. 25-37, 2006.

[26] F. Adams, The Genuine Works of Hippocrates, Baltimore: Williams and Wilkins, 1939. 
[27] E. E. Sterns, B. Zee, J. Sen Gupta and F. Saunders, "Thermography: Its relation to pathologic characteristics, vascularity, proliferative rate and survival of patients with invasive ductal carcinoma of the breast," Breast Cancer, vol. 77, pp. 1324-1328, 1996.

[28] A. J. Guidi and S. J. Schnitt, "Angiogenesis in pre-invasive lesions of the breast," The Breast $J$, vol. 2, pp. 364-369, 1996.

[29] P. Gamagami, "Indirect signs of breast cancer: Angiogenesis study," in Atlas of Mammography, Cambridge, Mass. Blackwell Science, 1996, pp. 231-326.

[30] T. Love, "Thermography as an Indicator of Blood Perfusion," Proc NY Acad Sci J, vol. 335, pp. 429-437, 1980.

[31] M. Gautherie, "Thermobiological Assessment of Benign and Malignant Breast Diseases," Am J Obstet Gynecol, vol. 147, no. 8, pp. 861-869, 1983.

[32] J. Gershen-Cohen, J. Haberman and E. Brueschke, "Medical thermography: A summary of current status," Radiol Clin North Am, vol. 3, pp. 403-431, 1965.

[33] H. Spitalier and D. Giraud, "Does Infrared Thermography Truly Have a Role in Present-Day Breast Cancer Management?," in Biomedical Thermology, New York, Alan R. Liss , 1982, pp. 269-278.

[34] J. Haberman, J. Francis and T. Love, "Screening a Rural Population for Breast Cancer Using Thermography and Physical Examination Techniques," Ann NY Acad Sci , vol. 335, pp. 492$500,1980$.

[35] M. Gautherie, "Thermobiological Assessment of Benign and Malignant Breast Diseases," Am J Obstet Gynecol, vol. 147, no. 8, pp. 861-869, 1983.

[36] K. Louis, J. Walter and M. Gautherie, "Long-Term Assessment of Breast Cancer Risk by Thermal Imaging," in Biomedical Thermology, NY, Alan R. Liss Inc, 1982, pp. 279-301.

[37] C. Gros and M. Gautherie, " Breast Thermography and Cancer Risk Prediction," Cancer, vol. 45, pp. 51-56, 1980.

[38] C. H. Jones, "Thermography of the Female Breast.," in Diagnosis of Breast Disease, Baltimore, University Park Press, 1983, pp. 214-234.

[39] I. Nyirjesy and Y. Ayme, "Clinical Evaluation, Mammography, and Thermography in the Diagnosis of Breast Carcinoma," Thermology, vol. 1, pp. 170-173, 1986.

[40] H. Useki, " Evaluation of the thermographic diagnosis of breast disease: relation of thermographic findings and pathologic findings of cancer growth," Nippon Gan Chiryo Gakkai Shi, vol. 23, pp. 2687-2695, 1988. 
[41] L. Thomassin and D. Giraud, "Detection of Subclinical Breast Cancers by Infrared Thermography," in Recent Advances in Medical Thermology, New York, Plenum Press, 1984, pp. 575-579.

[42] J. D. Wallace, "Thermographic examination of the breast: An assessment of its present capabilities," in Early Breast Cancer: Detection and Treatment, New York, American College of Radiology, Wiley, 1975., pp. 13-19.

[43] G. D. Dodd, "Thermography in Breast Cancer Diagnosis.," in Seventh National Cancer Conference, Los Angeles, 1972.

[44] R. P. Kedar and D. O. Cosgrove, "Breast carcinoma: measurement of tumor response in primary medical therapy with color doppler flow imaging," Radiol, vol. 190, pp. 825-830, 1994.

[45] I. Khalkhali and J. A. Cutrone, "Scintimammography: the complementary role of Tc-99m sestamibi prone breast imaging for the diagnosis of breast carcinoma," Radiol, vol. 196, pp. 421-426, 1995.

[46] M. Moskowitz, J. Milbrath and P. Gartside, " Lack of efficacy of thermography as a screening tool for minimal and Stage I Breast Cancer," N Engl J Med, vol. 295, pp. 249-252, 1976.

[47] J. C. Weinreb and G. Newstead, "MR imaging of the breast," Radiol, vol. 196, pp. 593-610, 1995.

[48] J. R. Keyserlingk, P. Ahlgren, E. Yu and N. Belliveau, "Infrared imaging of the breast: Initial reappraisal using high-resolution digital technology in 100 successive cases of Stage I and II breast cancer," The Breast Journal , vol. 4, pp. 245-251, 1998.

[49] E. F. J. Ring, "Progress in the measurement of human body temperature,", IEEE Eng. Med. Biol., vol. 17, no. 4, pp. 19-24, 1998.

[50] M. Molls and P. Vanupel, Blood perfusion and microenvironment of human tumours: Implications for Clinical radiooncology, Berlin: Springer-Verlag press, 1998.

[51] M. Zhou and Q. Chen, "Estimation of Temperature Distribution in Biological Tissue by Analytic Solutions to Pennes' Equation," in 2nd International Conference on Biomedical Engineering and Informatics, BMEI, IEEE, Nanjing, 2009.

[52] K. Yue, X. Zhang and F. YU, "An Analytic Solution of One-dimensional Steady-state Pennes' Bioheat Transfer Equation in Cylindrical Coordinates.,," Journal of Thermal Science, Vols. 13, Issue 3, pp. 255-258., no. 3, pp. 255-258, 2004. 
[53] H.-q. Yang, Q.-y. Lin, Z. Ye, S.-q. Chen and S.-s. Xie, "“Finite element thermal analysis of breast and its comparison with thermography," Proc of SPIE, vol. 6826, pp. 68260T1-T8, 2003.

[54] J. Liu and L. X. Xu, "Boundary information based diagontstics on the thermal states of biological bodies," Int. J. Heat Mass Transfer, vol. 43 , pp. 2827-2839, 2000.

[55] S. Hossain and F. A. Mohammadi, "Development of an Estimation Method for Interior Temperature Distribution in Live Biological Tissues of Different Organs," International Journal of Engineering and Applied Science, vol. 3, no. 2, pp. 55-65, 2013.

[56] S. Hossain and F. A. Mohammadi, "One-dimensional Steady-state Analysis of Bioheat Transfer Equation: Tumour Parameters Assessment for Medical Diagnosis Application," in International Multiconference on Enginnering and Technological Innovation, Florida, 2014.

[57] S. Hossain, E. T. Nezad and F. A. Mohammadi, "Neural network approach for the determination of heat Source parameters from the surface temperature image," in Canadian Conference on Electrical and Computer Engineering, Niagara Falls, 2011.

[58] N. M. Sudarshan, E. Y. K. Ng and S. I. The, " Surface temperature distribution of a breast with and without tumour," Computer methods in biomechanics and biomedical engineering, vol. 2, no. 3, pp. 187-199, 1995.

[59] E. Y. K. Ng and N. M. Sudharan, "Numerical uncertainty and perfusion induced instability in bioheat equation: its importance in thermographic interpretation," J Med Eng Tech, vol. 25, pp. 222-229, 2001.

[60] M. Mital and M. P. Ramana, "Breast Tumor Simulation and Parameters Estimation Using Evolutionary Algorithms," modeling and simulation in engineering, vol. 115, pp. 1-5 , 2008.

[61] S. Cotin, M. M. Delingette and N. Ayache, "Real time elastic deformation of soft tissues for surgery simulation," IEEE Trans. Vis. Comput. Graphics, vol. 5, pp. 62-73, 1999.

[62] M. M. Delingette, M. M. Doyle, B. W. Pogue, S. Jiang, J. Geng and K. D. Paulsen, "Breast deformation modeling for image reconstruction in near infrared optical tomography," Phys. Med. Biol., vol. 49, pp. 1131-1145, 2011.

[63] L. Davis, Handbook of genetic algorithms, New York: Van Nostrand Reinhold, 1991.

[64] A. Hopgood, Intelligent systems for engineers and scientists, New York: Library of Congress Cataloging , 2000.

[65] R. M. Lewis and V. Torczon, "Pattern Search Method for Linearly Constrained Minimization," SIAM J. OPTIM., vol. 10, no. 3, pp. 917-941, 2001. 
[66] R. M. Lewis and V. Torczon, "Pattern Search Method for Bound Constrained Minimization".

[67] H. H. Pennes, "Analysis of tissue and artier blood temperatures in the resting human forearm," J. Appl. Physiol, vol. 1, pp. 93-122, 1948.

[68] E. H. Wissler, "Pennes' 1948 paper revisited," The American Physilogical Society, vol. 1, pp. 35-41, 1998.

[69] T.-C. Shih, P. Yuan, W.-L. Lin and H.-S. Kou, "Analytical analysis of the Pennes' bioheat transfer equation with sinusoidal heat flux condition on skin surface," IPEM, Medical Engineering and Physics, vol. 29, no. 9, pp. 946-953, 2007.

[70] R. Romero, N. J.-L. Joel and M. G. F. J. Shen, "Analytical solution of the Pennes' equation for burn-depth determination from infrared thermographs," Mathematical Medicine and Biology, vol. 27, pp. 21-38, 2009.

[71] Introduction to COMSOL Multiphysics, The COMSOL , 2011.

[72] M. M. Osman and E. M. Afify, "Thermal modeling of the normal woman's breast," $J$. Biomech Eng., vol. 106, no. 2, pp. 123-130, 1984.

[73] A. R. McLean, M. E. Richards, C. S. Crandall and J. L. Marinaro, "Ultrasound determination of chest wall thickness: implications for needle thoracostomy," The American Journal of Emergency Medicine, vol. 29, no. 9, pp. 1173-1177, 2011.

[74] S. B. Lagundoye, "Subcutaneous Fat and Muscle Layers in Chest X-rays of Children with Kwashiorkor," Jornal of Tropical Pediatrics, vol. 1, pp. 287-290, 1974.

[75] D. N. Uyen, J. S. Brown, A. C. Isaac, J. Krycia and S. M. Mark, "Numerical evaluation of heating of the human head due to magnetic resonance imaging," IEEE Biomedical Engineering, vol. 51, no. 8, pp. 1301-1309, 2004.

[76] L. Jiang, W. Zhan and H. M. Lowe, "Modeling static and dynamic thermography of the human breast under elastic deformation," Phys. Med. Biol., vol. 56, no. 1, pp. 187-197, 2011.

[77] L. A. Priebe and A. J. Melch, "A dimensionless model for the calculation of temperature increase in biologic tissues exposed to nonionizing radiation," IEEE Trans. Biomed. Eng. , Vols. BME-26, pp. 244-250, 1979.

[78] H. Barcroft and O. G. Edholm, "The effect of temperature on blood flow and deep temperature in the human forearm," J. Physiol., vol. 102, pp. 5-20, 1942.

[79] G. Gutierrez, "Study of the bioheat equation with a spherical heat source for loal magnetic hyperthermia," in XVI Congress on numerical methods and their applications, Argentina, 2007. 
[80] T. Xianwu, "Relationship between surface temperature distribution and internal heat source size of the in-vitro tissues," in 26th Annual International Conference of the Engineering in Medicine and Biology Society, San Francisco, 2004.

[81] WiseGreek, "Muscular System - Muscles of the Human Body," muscle anatomy, [Online]. Available: http://1aled.fotomaps.ru/muscle-anatomy.php. [Accessed 08 September 2015].

[82] I. d. Santos, "Effect of variable heat transfer coefficient on tissue temperature next to a large vessel during radio frequency tumor ablation," Biomedical Engineering Online, pp. 7-21, 2008.

[83] A. S. Roger, B. C. Borman, A. C. David and F. Balash, "uyteufk hlwljg kllj khlklj khkh," hjgjkgi, 2003.

[84] Z. Minhua and C. Qian, "Estimation of temperature distribution in biological tissue by analytic solutions to Pennes equation," in 2nd International Conference on Biomedical Engineering and Informatics, Chaina, 2009.

[85] Z. Minhua and C. Qian, "Estimation of temperature distribution in biological tissue by analytic solution to Pennes equation," in 2nd International Conference on Biomedical Engineering and Informatics, Tianjin, 2009.

[86] G. Hébert, "Muscle et beauté plastique," 2000. [Online]. Available: https://en.wikipedia.org. [Accessed 8 September 2015].

[87] G. Chunfang, L. Kaiyang and Z. Shaoping, "A novel approach of analyzing the relation between the inner heat source and the surface temperature distribution in thermal texture maps," in 27th Annual Conference of the Engineering in Medicine and Biology, Shanghai, 2005.

[88] Chicago, 2012. [Online]. Available:

http://www.mcs.anl.gov/ anitescu/CLASSES/2012/LECTURES/S310-2012-lec9.pdf. [Accessed 30 July 2015].

[89] The Science of Natural Breast, "SAGGING BREASTS," [Online]. Available: http://thescienceofeating.com/. [Accessed 8 September 2015]. 


\section{Glossary}

\section{Parameter}

Thermal conductivity

Metabolism

Blood perfusion rate

Heat exchange rate

Specific heat (blood)

Specific heat (tissue)

Density (blood)

Density (tissue)

Artier blood temperature

Body core temperature

Skin temperature

Environment temperature

Tumor doubling time

Tumor diameter

Tumor radius

Tissue thickness or radius

Tumor location

Heat source model constant

Heat rate ratio

\section{Symbol}

$k$

$Q_{m}$

$\omega_{b}$

$h_{a}$

$c_{b}$

c

$\rho_{b}$

$\rho$

$T_{a}$

$T_{c}$

$T_{S}$

$T_{e}$

$\tau$

D

$R$

d

h

$\gamma$

n

\section{Unit}

$W /(m . K)$

$W / m^{3}$

$1 / s$

$W /\left(m^{2} . K\right)$

$J /(k g . K)$

$J /(k g . K)$

$\mathrm{kg} / \mathrm{m}^{3}$

$\mathrm{kg} / \mathrm{m}^{3}$

K

K

K

K

day

m

m

m

$\mathrm{m}$ 